Günter Silberer

\title{
Verhaltensforschung am Point of Sale
}

Ansatzpunkte und Methodik

Universitätsverlag Göttingen 

Günter Silberer

Verhaltensforschung am Point of Sale

This work is licensed under the Creative Commons License 2.0 "by-nd", allowing you to download, distribute and print the document in a few copies for private or educational use, given that the document stays unchanged and the creator is mentioned.

You are not allowed to sell copies of the free version.

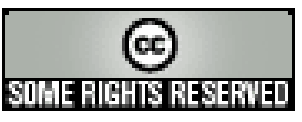


erschienen im Universitätsverlag Göttingen 2009 
Günter Silberer

\section{Verhaltensforschung am Point of Sale}

Ansatzpunkte und Methodik

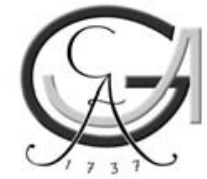

Universitätsverlag Göttingen 2009 


\section{Bibliographische Information der Deutschen Nationalbibliothek}

Die Deutsche Nationalbibliothek verzeichnet diese Publikation in der Deutschen Nationalbibliographie; detaillierte bibliographische Daten sind im Internet über $<$ http://dnb.ddb.de $>$ abrufbar.

Anschrift des Autors

Prof. Dr. Günter Silberer

Georg-August-Universität Göttingen

Platz der Göttinger Sieben 3

37073 Göttingen

Dieses Buch ist auch als freie Onlineversion über die Homepage des Verlags sowie über den OPAC der Niedersächsischen Staats- und Universitätsbibliothek (http://www.sub.uni-goettingen.de) erreichbar und darf gelesen, heruntergeladen sowie als Privatkopie ausgedruckt werden. Es gelten die Lizenzbestimmungen der Onlineversion. Es ist nicht gestattet, Kopien oder gedruckte Fassungen der freien Onlineversion zu veräußern.

Satz und Layout: Stefan Friedemann, Sascha Steinmann

Umschlaggestaltung: Margo Bargheer

(C) 2009 Universitätsverlag Göttingen

http://univerlag.uni-goettingen.de

ISBN: 978-3-940344-68-7 
A. Einleitung ..........................................................................................

B. Die Erfassung des Lauf-, Zuwendungs- und Kaufverhaltens............. 15

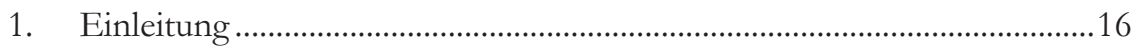

2. Allgemeines zum offenen Verhalten am POS............................................17

3. Zur Einteilung und Beurteilung der Erhebungsinstrumente ....................17

4. Die Erhebungsinstrumente im Einzelnen.................................................19

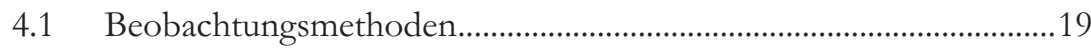

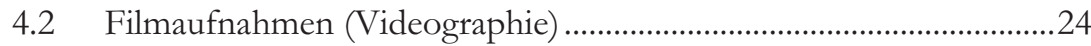

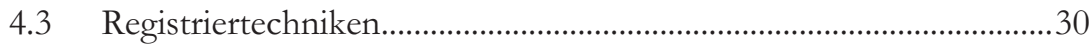

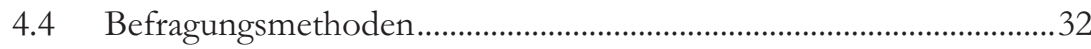

5. Auswählte Möglichkeiten der Methodenkombination...............................35

6. Die Sequenzanalyse als Datenauswertungsmethode ..................................38

7. Aktuelle und künftige Trends der POS-Forschung....................................40

8. Herausforderungen an die wissenschaftliche POS-Forschung.................43

C. Die Erfassung kognitiver Prozesse beim Ladenbesuch .................... 49

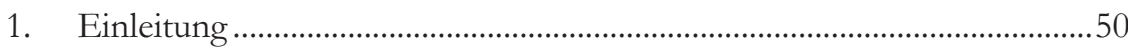

2. Mögliche Überlegungen eines Ladenbesuchers ........................................51

3. Mögliche Erfassungsmethoden im Überblick ...........................................53

4. Die relevanten Befragungsmethoden...........................................................54

5. Varianten der Denke-Laut-Methode............................................................56

6. Die gestützte Rekonstruktion kognitiver Prozesse....................................62

6.1 Varianten einer wirksamen Gedächtnisstütze ....................................62

6.2 Die videogestützte Gedankenrekonstruktion....................................63

7. Möglichkeiten einer Methodenkombination ..............................................67

7.1 Zum gruppeninternen Methoden-Mix ...............................................68

7.2 Zum gruppenübergreifenden Methoden-Mix ..................................69

8. Zusammenfassung und Ausblick .............................................................. 70 
D. Die Kaufbegleitung als Forschungsmethode........................................ 73

1. Einleitung............................................................................................... 74

2. Grundlegende Arten der Kaufbegleitung ................................................... 75

2.1 Die Kaufbegleitung als ethnographienahe Methode........................... 76

2.2 Die Kaufbegleitung als klassische Verhaltensforschung ................... 77

3. Die Kaufbegleitung als Methoden-Mix......................................................... 79

3.1 Der Methodeneinsatz in der Vorkaufphase ……………………….... 79

3.2 Der Methodeneinsatz bei der In-Store-Begleitung ……………….... 82

3.3 Der Methodeneinsatz beim Nachgespräch …………………………... 85

4. Zum derzeitigen Stand der Kaufbegleitungsforschung............................... 86

5. Zur künftigen Ausrichtung der Kaufbegleitungsforschung...................... 90

6. Zusammenfassung................................................................................... 98

E. Zur Selektivität der Verhaltensforschung am Point-of-Sale ................105

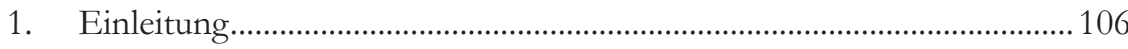

2. Selektivitätseffekte der Befragung und Beobachtung im Überblick..... 107

3. Zwei Studien zur Selektivität der Befragung und

Beobachtung von Ladenbesuchern ......................................................... 110

3.1 Zur Anlage der Studien........................................................................110

3.2 Befunde zur Selektivität der Befragung von Ladenbesuchern .......112

3.3 Befunde zur Selektivität der Beobachtung von Ladenbesuchern.116

3.4 Zusammenfassung der wichtigsten Ergebnisse .................................119

4. Folgerungen für die Verhaltensforschung am Point-of-Sale...................120 
F. Zur Reaktivität der Verhaltensforschung am Point-of-Sale 125

1. Einleitung 126

2. Reaktivitätseffekte in der Verhaltensforschung

3. Studien zur Reaktion von Ladenbesuchern auf die Analyse ihres Verhaltens.

3.1 Eine Studie zur Reaktion von Ladenbesuchern auf eine offene Beobachtung

3.2 Eine Studie zur Reaktion von Ladenbesuchern auf eine Befragung und Beobachtung

3.3 Eine Studie zur Reaktion von Ladenbesuchern auf die

Denke-Laut-Methode und die videogestützte

Gedankenrekonstruktion 151

3.4 Zusammenfassung der wichtigsten Ergebnisse

4. Folgerungen für die Verhaltensforschung am Point-of-Sale .154

G. Die abgestufte Verhaltensforschung am Point-of-Sale

1. Einleitung. 160

2. Dimensionen des Verhaltens von Ladenbesuchern und die Möglichkeiten ihrer Erfassung 161

3. Ein Drei-Stufen-Modell für die Verhaltensforschung am POS ............165

3.1 Erste Stufe: Registrierung des Kundenlaufs ....................................166

3.2 Zweite Stufe: Beobachtung des Zuwendungsund Kaufverhaltens

3.3 Dritte Stufe: Abfrage und Rekonstruktion kognitiver Prozesse ....173

4. Zusammenfassung und Ausblick 178 
H. Die Erforschung des Mitarbeiterverhaltens am POS ........................ 181

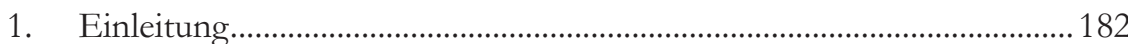

2. Verhaltensebenen und Verhaltensbereiche .............................................. 182

3. Erfassungsmethoden und Bewertungskriterien im Überblick ................184

4. Einzelne Erfassungsmethoden und deren Bewertung ............................ 185

4.1 Beobachtungsmethoden und deren Eignung.....................................186

4.2 Registrierungsmethoden und deren Eignung......................................190

4.3 Videographische Methoden und deren Eignung.............................. 191

4.4 Befragungsmethoden und deren Eignung .........................................192

4.5 Die gestützte Erinnerung des Verhaltens ...........................................194

5. Zum simultanen und konsekutiven Methoden-Mix ..................................195

6. Zusammenfassung und Ausblick ...............................................................196 
A

Einleitung 
Der Point-of-Sale hat mit dem verschärften Wettbewerb auf den Konsumgütermärkten in den letzten Jahren an Bedeutung gewonnen. Dies gilt nicht nur für den Wettbewerb der Händler und jener Anbieter, die ihre Produkte über eigene Verkaufsstellen vertreiben, sondern auch für jene Hersteller, die ihre Produkte über den Handel vertreiben. So wichtig das Verhalten der Betreiber von Verkaufspunkten, die Gestaltung des Sortiments, der Preise, der Kommunikation und der Öffnungszeiten, auch des Einsatzes von Mitarbeitern, so wichtig ist auch das Verhalten der Besucher bzw. der potentiellen Käufer. Vielen Hinweisen aus der Praxis und aus der Wissenschaft zufolge werden nach wie vor viele Kaufentscheidungen erst am Point-of-Sale (POS) getroffen. Selbst wenn der Kauf eines bestimmten Produktes schon vor dem Besuch eines Verkaufspunktes beschlossen ist, müssen weder die Marke noch der konkrete Artikel noch die Anzahl der Artikel feststehen.

Mit der Bedeutung des Verhaltens der Ladenbesucher vor Ort steigt auch die Bedeutung der Erforschung dieses Verhaltens. Zwar liefern Scannerkassen und vergleichbare Systeme an vielen Orten zahlreiche und verlässliche Daten über die Abverkaufserfolge und damit über die gekauften Produkte; auch über die Zusammensetzung von Warenkörben; sie sagen aber nichts darüber aus, wie die diese Käufe zustande gekommen sind, welche Kaufpläne vorher geschmiedet wurde, welche Kaufpläne verschoben oder ganz aufgegeben wurden, und welche Produktkontakte im Laden zustande kamen, was dem Besucher dabei durch den Kopf ging und welche Käufe gar nicht geplant waren. Um dieses Verhalten in Erfahrung zu bringen, bedarf es einer gezielten Verhaltensforschung am POS. Und die Aussagekraft entsprechender Ergebnisse hängt nicht nur vom Gegenstand der Analysen, sondern auch vom Einsatz geeigneter Methoden ab.

Mit dem Einsatz diverser Methoden bei unterschiedlichen Fragestellungen wollen wir uns im Folgenden näher befassen. Dafür gibt es mehrere Gründe: Zum einen ist die Eignung klassischer Methoden beim Einsatz am POS nicht gerade 
häufig, auch gar nicht systematisch untersucht worden. Zum anderen haben sich in den letzten Jahren aufgrund des technischen Forschritts, vor allem der Digitalisierung, Miniaturisierung und der Entwicklung von Kommunikationsnetzen, neue Möglichkeiten ergeben, das Verhalten am POS zu analysieren. Und diese Möglichkeiten sind bis heute nur teilweise erkannt und genutzt worden; auch deren Eignung konnte bislang nur selten zum Gegenstand gezielter Studien avancieren.

Im Folgenden soll nun aufgezeigt werden, wie eine entwickelte Verhaltensforschung am POS aussehen kann, welche Ansatzpunkte dabei in Frage kommen und welche Forschungsinstrumente zum Einsatz kommen können. Des Weiteren interessieren der kombinierte Einsatz von Methoden und die mögliche Abfolge des Methodeneinsatzes im Rahmen einer Forschungsstrategie. Im Vordergrund steht dabei die Frage nach der Eignung einzelner Forschungsmethoden, das zu messen was gemessen werden soll, also deren interne Validität, und die Frage nach der Übertragbarkeit von Befunden auf vergleichbare Situationen und Populationen, in bzw. bei denen keine Messungen durchgeführt werden, also die Frage nach der externen Validität von Befunden. Empirische Studien befassen sich mit der internen Validität ebenso wie mit der externen, deshalb auch mit der Frage, welches Ausmaß die Selektivität und die Reaktivität der teilnehmenden, analysierten Probanden annimmt. Ein Großteil dieser Studien, über die zu berichten sein wird, wurde von der Arbeitsgruppe „Verhaltensforschung am POS“ am Institut für Marketing und Handel der Universität Göttingen durchgeführt. Diese befassten sich nicht nur mit dem Nachfragerverhalten im stationären Handel, sondern auch mit dem Surferverhalten im elektronischen Shop, in dem ein Hersteller oder ein Händler oder beide ihre Produkte online vertreiben.

Im Folgenden wollen wir uns auf die Erfassung des Nachfrager- bzw. Besucherverhaltens im stationären Handel konzentrieren. Ein Beitrag beschäftigt sich allerdings auch mit der Erfassung des Mitarbeiterverhaltens und öffnet den Blick auf die Mitarbeiter-Kunden-Interaktion am POS. Über Studien zum 
Surferverhalten im eShop und zu den dabei einsetzbaren Methoden ist an anderer Stelle zu berichten (vgl. dazu die Abhandlung von Silberer (1997) und die Studien von Heimbach (1999/2001), Silberer et al. (2002, 2003), Engelhardt (2004/2006), Mau (2008) und Schulz (2008) als Beispiele). Im stationären Handel werden nach wie vor die meisten Konsumgüter eingekauft, auch wenn der Online-Kauf kontinuierlich an Bedeutung gewinnt. Hierbei sei aber auch festgehalten, dass sich die Möglichkeiten einer Verhaltensforschung im stationären POS von den Möglichkeiten einer Verhaltensforschung im Web deutlich unterscheiden.

Im Folgenden werden wir uns auch allein auf die Ansatzpunkte und Methodik einer Verhaltensforschung am POS konzentrieren. Dies bedeutet, dass die Versuche, das Verhalten am POS zu erklären und die Folgen dieses Verhaltens einzuschätzen, in dieser Arbeit nicht zur Sprache kommen werden. Damit sei kein Plädoyer zugunsten einer rein deskriptiven Verhaltensforschung ausgesprochen. Ganz im Gegenteil: Vornehmliches Ziel aller Verhaltensstudien muss darin bestehen, das faktische Verhalten nicht zu erfassen, sondern auch zu verstehen und in seinen Folgen richtig abzuschätzen. Und dieses Ziel kann künftig umso schneller erreicht werden, je besser es gelingt, das zu erklärende, faktische Verhalten am POS erst einmal richtig zu erfassen bzw. richtig einzuschätzen.

\section{Literatur}

Engelhardt, J.-F. (2004/2006).

Kundenlauf in elektronischen Shops. Typologisierung und Analyse des Erlebens und des Blick-, Click- und Kaufverhaltens, Dissertation Universität Göttingen, Wirtschaftswissenschaftliche Fakultät, Hamburg: Dr. Kovac

Heimbach, P. (1999/2001).

Nutzung und Wirkung interaktiver Werbung. Eine Studie zum Blickverhalten im Internet, Dissertation Universität Göttingen, Wirtschaftswissenschaftliche Fakultät, Wiesbaden: Gabler Edition Wissenschaft

Mau, G. (2008).

Die Rolle der Emotionen beim Besuch von Online-Shops, Dissertation Universität Göttingen, Wirtschaftswissenschaftliche Fakultät (in Vorbereitung) 
Schulz, S. (2008).

Der Einfluss der Usability, des Risikos und des Kundenstatus auf den Surfprozess sowie die Auswirkungen auf das Vertrauen, Dissertation Universität Göttingen, Wirtschaftswissenschaftliche Fakultät

Silberer, G. (1997).

Medien- und Rechnergestützte Interaktionsanalyse (MERIAN), in: G. Silberer (Hg.). Interaktive Werbung. Marketingkommunikation auf dem Weg ins digitale Zeitalter, Stuttgart: Schaeffer-Poeschel, S. 337-358

Silberer, G., Engelhardt, J.-F. \& Wasmuth, N. (2002).

E-Shop-Merkmale aus Kundensicht - Ergebnisse einer Conjoint-Analyse, in: i-com

- Zeitschrift für interaktive und kooperative Medien, 1. Jg. (2002), Heft 2, S. 24-30

Silberer, G., Engelhardt, J.-F. \& Wilhelm, T. (2003).

Der Kundenlauf im Web-Shop bei unterschiedlicher Besuchermotivation, Beitrag zur Marketingforschung No 5, Universität Göttingen: Institut für Marketing und Handel 

B

Die Erfassung des Lauf-, Zuwendungs- und Kaufverhaltens 


\section{Einleitung}

Viele Kaufentscheidungen werden erst am POS getroffen. Das Verhalten der (potentiellen) Käufer bzw. Kunden am Point-of-Sale (POS) entscheidet über den Erfolg des Anbieters, aber auch über den Erfolg des Käufers. Deshalb ist das Käuferverhalten vor Ort ein zentraler Erfolgsfaktor im Marketing. Dies gilt für alle Verkaufspunkte, für den stationären Handel ebenso wie für elektronischen Handel, auch für den Direktvertrieb in Filialen oder Niederlassungen. Im Folgenden wollen wir uns auf das Käuferverhalten im Ladengeschäft bzw. im stationären Handel konzentrieren. Hier werden auch im Internetzeitalter nach wie vor viele Produkte verkauft und gekauft.

Des Weiteren werden wir uns auf das beobachtbare, offene Verhalten der Ladenbesucher beziehen. Dabei liegt es nahe, in erster Linie an die Mitnahme bzw. and den Kauf von Produkten zu denken, stehen doch die Verkaufsziele im Vordergrund der Handelsziele. Bei näherer Betrachtung stellt sich jedoch heraus, dass Abverkaufszahlen allein wenig darüber aussagen, mit welchen Angeboten der Besucher in Kontakt gekommen ist, ohne sie zu wählen, und mit welchen Angeboten er nicht in Kontakt gekommen ist, bei denen eine Kaufbereitschaft durchaus bestehen hätte können. Vor diesem Hintergrund liegt es nahe, wie bei Werbemaßnahmen auch am POS nach den Kontakten zu fragen, die zwischen dem Besucher und dem Angebot, aber auch zwischen dem Besucher und den Mitarbeitern des Geschäftes zustande gekommen sind. Für die Verhaltensforschung vor Ort bedeutet dies, dass sich ein Anbieter nicht allein für die Abverkaufsdaten interessieren sollte, sondern auch für das Lauf- und Zuwendungsverhalten der Ladenbesucher.

Das Laufverhalten der Ladenbesucher entscheidet vor allem über die Anzabl der Kontakte mit dem Angebot und das Zuwendungsverhalten entscheidet vor allem über die Qualität dieser Kontakte. Deshalb werden uns in diesem Beitrag das Laufund das Zuwendungsverhalten als offenes Verhalten der Besucher besonders interessieren. Dabei geht es in erster Linie um die Möglichkeiten, dieses Verhalten 
zu erfassen. Neben den klassischen Erfassungsmethoden werden auch neuere Methoden zur Sprache kommen. Die Analyse der Ursachen und der Wirkungen des Verhaltens am POS bleibt einem anderen Beitrag vorbehalten.

\section{Allgemeines zum offenen Verhalten am POS}

Das offene Verhalten, das ein Besucher im Ladengeschäft an den Tag legen kann, ist vielfältig. Zu den verschiedenen Verhaltenskategorien zählen

(1) das Laufverhalten bzw. die Laufwege, auch „way finding“ genannt (siehe Weismann 1981, O’Neill 1992, Titus \& Everett 1996),

(2) die angebotsbezogene Zuwendung, vor allem das Anschauen, Anfassen, Zurücklegen und Mitnehmen von Ware, und

(3) das sonstige Verhalten, so z.B. die Nutzung gastronomischer Angebote, der

Umtausch von Ware und die Rückgabe von Leergut.

Die angebotsbezogene Zuwendung zum Angebot kennt zwei grundlegende Varianten, zum einen das Verhalten von Besuchern, die bestimmte Produkte kaufen wollen, zum anderen das Stöbern, bei dem die Besucher den Laden ohne Kaufabsicht betreten, um sich umzuschauen, sich zu informieren oder sich anregen zu lassen (vgl. Babin \& Darden 1995, Bloch \& Richins 1983, Iyer 1989, Kahn \& Schmittlein 1992, Zielke 2002).

Darüber hinaus lassen sich auch unterschiedliche Verhaltensbereiche unterscheiden. Verhaltensbereiche sind z.B. der der einzelne Laden oder das Gemeinschaftswarenhaus oder das Einkaufszentrum, in dem sich viele Geschäfte versammeln. Darüber hinaus macht es einen Unterschied, ob es um einzelne Besuche zu einem bestimmten Zeitpunkt oder um wiederholte Ladenbesuche in einem bestimmten Zeitraum geht. Die Analyse zeitraumbezogenen Verhaltens steigert die Chance, sog. Verhaltensmuster zu erkennen.

\section{Zur Einteilung und Beurteilung der Erhebungsinstrumente}

Der Blick auf das Methodenarsenal der Verhaltensforschung, aber auch auf bislang realisierte Verhaltensstudien am POS macht schnell deutlich, dass mehrere 
Erhebungsinstrumente in Frage kommen (vgl. Granbois 1968 S. 28). Es lassen sich folgende Methodenbündel unterscheiden: (1) die Beobachtungsmethoden, Erhebungen mit dem Einsatz von Beobachtern, sei es eine teilnehmende oder eine nichtteilnehmende Beobachtung, eine offene oder eine verdeckte Beobachtung, (2) Filmaufnahmen bzw. die Videographie, die Aufzeichnung des Lauf- und/oder Zuwendungsverhaltens mit sog. Filmkameras, (3) die Registrierungstechniken, bei denen weder Personen noch Kameras zum Einsatz kommen, so z.B. das Einrichten von Lichtschranken, und (4) die Befragungsmethoden, bei denen die Probanden darum gebeten werden, über ihr Verhalten im Geschäft schriftlich oder mündlich Auskunft zu geben.

Da bei einer Erfassung des offenen Verhaltens der Ladenbesucher immer auch die Eignung der relevanten Erfassungsmethoden interessiert, seien hier vorab die wichtigsten Beurteilungskriterien aufgelistet und erläutert: die Realisierbarkeit, die interne Validität und die externe Validität.

Bezüglich der Realisierbarkeit ist nicht nur auf das finanziell, personell und technisch Machbare zu achten, sondern auch auf die soziale Akzeptanz bei allen Beteiligten und Betroffenen - bei den Probanden selbst, bei den anderen Ladenbesuchern, bei den Mitarbeitern und Mitarbeiterinnen und dem Management. Die ausgewählten bzw. angesprochenen Ladenbesucher müssen einer Erhebung im Vorhinein oder im Nachhinein zustimmen. Besteht die Gefahr, dass andere Ladenbesucher als unbeteiligte Dritte aufgrund der Beobachtung und/oder Befragung anderer Ladenbesucher verunsichert werden, sind diese ggf. aufzuklären. Sollten sich Verkaufs- und Servicemitarbeiter durch die Beobachtung oder durch Filmaufnahmen ebenfalls beobachtet bzw. aufgezeichnet fühlen, müssen sie bzw. ihre Vertretung um Zustimmung gebeten werden. Dass auch die Akzeptanz des Managements erforderlich ist, versteht sich von selbst. Dabei ist zu bedenken, dass das Management eine Studie nicht nur im Blick auf die angestrebten Erkenntnisse beurteilen sollte, sondern auch im Hinblick auf mögliche Bedenken seitens der Kunden und der Mitarbeiter. 
Ein weiteres Beurteilungskriterium ist die interne Validität. Gemeint ist damit die Eignung im engeren Sinne, also ob ein Instrument bei einem sachgerechten Einsatz genau das erfasst, was es erfassen soll. Folgende Validitätskriterien können in Betracht gezogen werden: das Urteil von Experten (Augenscheinvalidität), die Übereinstimmung mit einem empirisch erfassten Außenkriterium (Kriteriumsvalidität), mit einer mehrfach getesteten und bewährten Messtheorie (nomologische Validität) oder mit Messungen anhand eines bewährten Messinstruments (Konvergenzvalidität). Bei der Formulierung von Ansprüchen an die interne Validität ist darauf zu achten, welche Aspekte eines Verhaltens erfasst werden sollen; gelegentlich werden nämlich Messinstrumente völlig zu Unrecht mit dem Argument kritisiert, sie könnten nicht alle Aspekte des POS-Verhaltens abbilden.

Schließlich ist auch die externe oder ökologische Validität zu beachten, denn eine Messung des Besucherverhaltens sollte in aller Regel gewisse Rückschlüsse auf das Verhalten vergleichbarer Ladenbesucher in ähnlichen Geschäften und vergleichbaren Situationen erlauben. Dies gilt auch für den Schluss auf jene Situationen, in denen keine Erhebungen durchgeführt werden. Vor diesem Hintergrund ist die Forderung von Wells \& LoSciuto (1966 S. 28f.) zu sehen, dass Stichproben repräsentativ sein, alle Aspekte eines Ladens abdecken und außerdem unterschiedliche Zeiten berücksichtigen sollten. Ob ein derart hoher Anspruch an die ökologische Validität einer Erhebung immer geboten, geschweige denn zu realisieren ist, darf bezweifelt werden. Auch jene Erkenntnisse, die personen-, raum- und zeitbezogen zu relativieren sind, können durchaus hilfreich, wenn nicht gar wertvoll sein.

\section{Die Erhebungsinstrumente im Einzelnen}

\subsection{Beobachtungsmethoden}

Das Lauf- und Zuwendungsverhalten am POS ist ein offenes Verhalten, so dass es nahe liegt, als erstes an die Beobachtung als Erhebungsmethode zu denken. Die 
Tatsache, dass per Beobachtungen keine psychischen Vorgänge wie z.B. Kaufpläne und deren Revision erfasst werden können (so z.B. Granbois 1968 S. 29), ändert nichts an der Eignung der Beobachtungsmethode bei der Erfassung offenen Verhaltens. Kennzeichnend ist der Einsatz geschulter Beobachter. Diese können sich passiv verhalten (passive bzw. nicht-teilnehmende Beobachtung) oder sich aktiv am Geschehen beteiligen (teilnehmende Beobachtung). Und den beobachteten Personen kann der Umstand der Beobachtung vorenthalten (verdeckte Beobachtung), aber auch bewusst gemacht bzw. vorher mitgeteilt werden (offene Beobachtung).

\section{(1) Die verdeckte, passive Beobachtung}

Bislang durchgeführte Studien setzten überwiegend auf die verdeckte, passive Beobachtung. Klassische Beispiele liefern die US-amerikanischen Studien von Wells \& LoSciuto (1966) und Granbois (1968), in denen der Kundenlauf am POS beobachtet wurde. Wells \& LoSciuto (1966, S. 227) konzentrierten sich auf das Verhalten in einzelnen Gängen, sog. Episoden, und dabei nur auf solche Warenbereiche, für die der betreffende Besucher ein Kaufinteresse zu erkennen gab. Die Beobachter hatten die Aufgabe, das Anschauen, Zurücklegen und Mitnehmen von Produkten $\mathrm{zu}$ erfassen, auch $\mathrm{zu}$ registrieren, worauf geachtet wurde (z. B. Gewicht und Preis) und wer auf wen Einfluss ausübte. Ob eine valide Erfassung konkreter Zuwendungsobjekte gelang, wird nicht berichtet (S.232). Granbois (1968, S.29) setzte Beobachter im typischen Mitarbeiter- bzw. Verkäuferoutfit ein, die den Kundenlauf in Ladengrundrisse (maps of the store's layout) einzutragen hatten, auch Punkte, an denen Produkte angeschaut und/oder mitgenommen wurden. Von insgesamt 388 beobachteten Ladenbesuchern hatte nur eine Person die Beobachtung bemerkt. Ähnliche Erfolge berichten Botelho (2002, S.26f.) und Baldauf et al. (1997, S.106f.) von ihren Kundenlaufstudien in deutschen bzw. Österreichischen Geschäften. In der breit angelegten ISB-Studie (1986, S.13) wurden offenbar recht viele Beobachter von beobachteten Personen 
als solche wahrgenommen. Die Autoren berichten von 819 erfolgreichen Beobachtungen bei „ca. 1.000 Beobachtungsversuchen“.

Neben dem Kundenlauf lassen sich auch bestimmte Formen der Zuwendung beobachten. Dennoch sind nur wenige Versuche in dieser Richtung zu finden. Bei der Beobachtung des Zuwendungsverhaltens an einem Regal setzte Hoyer (1984, S.825) einen Beobachter in Mitarbeiterkleidung ein, der den Besucher unauffällig zu beobachten und festzustellen hatte, welche Marken betrachtet, entnommen und gekauft werden und wie viel Zeit dafür benötigt wird. Von Interesse waren auch Vergleichsprozesse: across-brand comparisons und within-brand comparisons (ebd). Da solche Prozesse bei kleinformatigen Produkten keine sichtbaren Bewegungen induzieren und somit per Beobachtung schlecht zu erfassen sind, hatte sich Hoyer für eine Beobachtung am Waschmittelregal und damit für die Zuwendung bei großformatigen Produkten entschieden.

Da visuelle Zuwendungen am Regal i. d. R. nicht gut zu beobachten sind, wenn der Beobachter hinter dem Probanden steht, liegt es nahe, eine Beobachtung von Personen, die hinter dem Regal stehen, zu ermöglichen. Zu diesem Zwecke sind gelegentlich sog. Einwegspiegel als Regalwand eingesetzt worden. Dieses Verfahren kann aber nur gelingen, wenn die Produkte, die in den Regalen stehen, die Sicht auf die Regalbesucher nicht verbauen.

\section{(2) Die offene, passive Beobachtung}

Eine Beobachtung erfolgt offen z.B. dann, wenn die Erlaubnis vorab erbeten und von den zu beobachtenden Personen erteilt worden ist. Verhält sich die als Beobachter bekannte Person passiv, so ist deren Einfluss auf das Geschehen vielleicht gering, vielleicht aber auch erheblich, da die beobachtete Person allein schon auf den Umstand den Beobachtung reagieren kann. Eine Zustimmung zur Beobachtung und die Reaktivität im Verhalten schließen sich keineswegs aus. Studien, in denen Ladenbesucher offen, aber passiv beobachtet wurden, sind meines Wissens bislang kaum durchgeführt worden. 


\section{(3) Die teilnehmende Beobachtung}

Ein Beobachter, der am Geschehen aktiv teilnimmt, kann den beobachteten Personen bekannt sein, muss es aber nicht. Wenn z.B. das Verkaufspersonal an Fremdbedienungstheken mit der Beobachtung von Kunden betraut ist, muss dies den Ladenbesuchern nicht mitgeteilt werden. In solchen Fällen wäre zu bedenken, dass die beteiligten Mitarbeiter als verdeckte, teilnehmende Beobachter nicht immer objektiv urteilen wollen. Sinnvoller wäre es daher, die Interaktion zwischen dem Verkaufs- und Servicepersonal einerseits und Besuchern andererseits durch Dritte beobachten zu lassen (zur sozialen Interaktion am POS siehe Bitner 1992 S. 61f.).

Sollen Beobachter die ausgewählten Personen während des Ladenbesuchs begleiten, kann dies durchaus offen und auch mit dem Auftrag geschehen, das Verhalten des Ladenbesuchers zu beeinflussen. In der Studie von Payne \& Ragsdale (1978) wurden 19 Hausfrauen bei mehreren wöchentlichen Einkäufen begleitet (S. 572f.). Die Begleiter hatten im „candy department“ Folgendes zu beobachten und festzuhalten: den Kundenlauf, die Mitnahme von Produkten, die Benutzung eines Einkaufszettels und den Einsatz von Coupons (S. 572f.). Zeitgleich hatten sie als aufmerksame Begleiter dafür zu sorgen, dass die Probanden ihre Gedanken laut und verständlich artikulieren und die Artikulation der Gedanken nicht abreißt („Denke-laut-Methode“). Eine solche Doppelaufgabe hatten auch die „trainierten“ Beobachter in der Studie von Titus \& Everett (1996 S. 267f.). Sie erhielten den Auftrag, Besucher durch den Laden zu begleiten, deren Suchverhalten zu beobachten und deren Gedankenartikulation zu stimulieren. $\mathrm{Zu}$ beobachten waren Probanden, deren Aufgabe darin bestand, die auf einer Einkaufsliste aufgeführte Produkte (insgesamt 21) im Geschäft zu finden, aber nicht zu kaufen.

„Shopping With the Consumer“ - eine besondere Variante der teilnehmenden Beobachtung - entwickelten und empfehlen Otnes et al. (1995) bzw. Lowrey et al (2005). Hier wird der Käufer nach vorheriger Vereinbarung und Befragung bei mindestens zwei Einkäufen begleitet. Er soll spätestens beim zweiten 
Geschäftsbesuch mit dem Umstand der Begleitung vertraut sein und sich so natürlich wie möglich verhalten können (Lowrey et al. 2005 S. 177f.). Diesem Ziel dient auch die Aufklärung der Probanden - ,so participants would not be alarmed during the actual trip" (S. 177).

\section{Beurteilung der Beobachtungsmethoden:}

Die technische Machbarkeit stellt bei der Beobachtung kein nennenswertes Problem dar. Allerdings sind Beobachtungen ziemlich aufwendig. Die Beobachter müssen sorgfältig eingewiesen und ausreichend geschult werden (Lowrey et al. 2005). Ggf. sind gemeinsame Geschäftsbesuche rechtzeitig zu planen und zu vereinbaren. Probleme bereitet eher die soziale Akzeptanz. Nicht jeder Käufer möchte beobachtet und erst recht nicht begleitet werden. Widerstände hängen nicht nur von der Ansprache und dem Geschlecht der Begleitperson, sondern auch davon $\mathrm{ab}$, welchen Zwecken die Studie dient (ob wissenschaftlichen oder kommerziellen Zwecken). Von daher ist es zumindest bei offenen und teilnehmenden Beobachtungen schwierig, eine Selektivität der Stichprobe und somit eine Reduktion der externen Validität zu vermeiden. Letztere leidet auch unter dem Einfluss, den die Beobachtung auf die Kaufsituation und damit auf das POS-Verhalten ausüben kann und tatsächlich ausübt. Nach unseren Erfahrungen zu urteilen, gibt es in der Tat sogenannte Gewöhnungseffekte, die sich nicht nur beim zweiten beobachteten Kauf, sondern auch schon während des ersten oder gar einzigen beobachteten Geschäftsbesuchs einstellen. Die entscheidende interne Validität einer Beobachtung von Geschäftsbesuchern hängt vor allem von der Eignung der Beobachter ab, zum einen von ihren vorhandenen Fähigkeiten (vor allem ihrer sozialen Intelligenz), zum anderen von ihrer Einweisung und dem Training. Trainiert werden muss das Beobachten selbst, aber auch das Festhalten der Beobachtungsergebnisse, sei es auf einem Papier, sei es auf einem „tablet-PC“. Außerdem ist Lowrey et al. (2005 S. 183) zuzustimmen, wenn sie betonen, dass „learning-by-doing“ auf jeden Fall hinzukommen sollte. 


\subsection{Filmaufnahmen (Videographie)}

Videoaufnahmen eignen sich ebenfalls für die Erfassung des Verhaltens am POS. Man kann dabei von einer kameragestützten Beobachtung sprechen, aber auch von einer Art Registrierung, wenn kein Kameramann im Einsatz ist. Jedenfalls gibt es verschiedene Möglichkeiten, die Videokamera am POS einzusetzen.

\section{(1) Aufnahmen mit Raumkameras}

In vielen Geschäften sind Raumkameras aus Sicherheitsgründen installiert. Sie dürfen jedoch nicht zu Marktforschungszwecken eingesetzt werden. Newman \& Foxall (2003, S. 592f.) plädieren dafür, Raumkameras für die Erfassung des Kundenlaufs so zu nutzen, dass keine rechtlichen und ethischen Schranken verletzt werden. Dies könne dadurch geschehen, dass ein zentraler Rechner die von digitalen Kameras aufgenommenen Videos erstens speichert, zweitens per Musterkennung auswertet und so das Lauf- und Zuwendungsverhalten der Besucher erfasst sowie drittens die zuordenbaren Gesichter unkenntlich macht. $\mathrm{Ob}$ mit einem solchen System eine raumübergreifende Erfassung des individuellen Kundenlaufs gelingt und wie teuer ein System zu stehen käme, das diese schwierige Trackingaufgabe über mehrere Räume hinweg meistern kann, bleibt allerdings offen. Erste Anwendungen bei Newman \& Foxall (2003) zeigen, dass die Aufzeichnung von Laufwegen in einem bestimmten Raum und die Anonymisierung der involvierten Mitarbeiter gelingen kann (S. 593f.). Allerdings sind auch bei einem Tracking von Besuchern, das sich auf einen einzelnen Raum beschränkt, enge Grenzen gesetzt: die im jeweiligen Raum installierten Kameras müssen in der Lage sein, alle Stellen der Warenpräsentation und damit jedes denkbare Zuwendungsverhalten zu erfassen. Dies dürfte in vielen Fällen an hohen Regalen und bestimmten räumlichen Verhältnissen scheitern. 


\section{(2) Aufnahmen eines Kameramanns}

Eine Erfassung von Lauf- und Zuwendungsaktivitäten kann gelingen, wenn der Besucher von einem Kameramann „begleitet“ wird, der versucht, alle wichtigen Aspekte des Lauf- und Zuwendungsverhaltens $\mathrm{zu}$ filmen (vgl. Silberer 2005, Büttner et al. 2005). Wenn es darum geht, dieses Filmen für Dritte so unauffällig wie möglich zu praktizieren, bieten die modernen, handlichen, digitalen Filmkameras sehr gute Möglichkeiten. Erste Erfahrungen mit diesem Verfahren zeigen, dass ein behutsamer, unauffälliger Einsatz kleiner digitaler Filmkameras möglich und die Akzeptanz vor allem bei jüngeren Generationen und bei aufgeschlossenen Führungskräften vorhanden ist (s. Silberer 2005, S.268). Eine unmittelbar anschließende Filmvorführung am PC eröffnet die Möglichkeit, mit der betreffenden Person jene Lauf- und Zuwendungsaktivitäten zu klären, z.B. die Art und Ausrichtung einer Zuwendung am Regal, bei denen der Film allein keine hinreichenden Anhaltspunkte liefert. Da nicht alle Ladenbesucher einer derartigen Begleitung, Aufzeichnung und Videoauswertung zustimmen werden, ist festzuhalten, dass der diagnostische Gewinn bei dieser Verhaltensanalyse mit Einschränkungen bei der Repräsentativität der Stichprobe „erkauft“ werden muss. Auch muss bei einem solchen Vorgehen eine gewisse Reaktivität der Probanden in Kauf genommen werden.

\section{(3) Standortgebundene Filmaufnahmen}

Bei den standort-, z.B. regalgebundenen Filmaufnahmen, lassen sich zwei Varianten unterscheiden: Aufnahmen in Richtung Regalfront und Aufnahmen aus der Regalwand heraus. Norman Albers (zitiert bei Hicks \& Kohl, 1955) arbeitete schon früh mit einer Memomotion- bzw. Zeitraffer-Kamera, um das Verhalten der Kunden am Milchregal hinweg zu erfassen. Die Kamera war über drei Wochen in der Nähe (an einem anderen Regal) angebracht und konnte das gesamte Milchregal aufnehmen. Auf diese Weise gelang es, Hinweise auf die Käufer (Geschlecht, Altersgruppe) und deren Verhalten, nämlich deren Markenzuwendung und deren 
Produktmitnahme, zu gewinnen (S.169f.). Als Vorteile dieses Verfahrens heben Hicks \& Kohl (1955 S.170) nicht nur die genauen Zeitangaben, den möglichen Dauerbetrieb (als eine Art Vollerhebung) und die niedrigen Kosten hervor, sondern auch die Möglichkeit, das Besucherverhalten „ohne Störeffekte“ zu erfassen.

Gelegentlich werden hinter einem Regal Einwegspiegel aufgebaut, um so das Kundenverhalten unbemerkt beobachten bzw. filmen zu können. Russo \& Leclerc (1994) arbeiteten mit einer Videokamera hinter dem Regal, hinter einem solchen Einwegspiegel (One-Way Mirror). Auf diese Weise wollten sie das Wahrnehmungsverhalten der Besucher als visuelle Zuwendung erfassen. Die Kunden sollten sich auf einen ganz bestimmten Punkt stellen, damit die erforderlichen, auswertbaren Aufnahmen gelingen (S. 275f.). Die Auswertung der Videoaufnahmen erlaubte eine Bestimmung der Blickfixationen und ihrer Sequenz, nicht aber die Dauer einzelner Fixationen (S. 276). Von 47 Probanden hatte nur eine Person den Einwegspiegel bemerkt, war aber nicht misstrauisch geworden (S. 276).

\section{(4) Aufnahmen einer „Augenkamera“ (Blickregistrierung)}

Eine klassische Technik für die Erfassung der visuellen Zuwendung - De Heer et al. (2000) sprechen vom „exploratory visual search behavior“ - ist der Einsatz einer sog. „Augenkamera“. Diese Technik der Blickregistrierung besteht aus zwei Teilsystemen: zum einen aus der Aufzeichnung des Blickfeldes anhand einer extrem kleinen Videokamera (Erzeugung des Blickfeldvideos), zum andern aus der Erfassung des Blickverlaufs, d.h. der Fixationen und der Saccaden. Beide Systeme müssen im konkreten Anwendungsfall kalibriert werden. Allerdings sind die Anforderungen an die Erstellung von Blickvideos, die das Blickfeld richtig wiedergeben, nicht so hoch wie die Anforderungen an die Aufzeichnung von Blickverläufen. Bei Personen mit voller Bewegungsfreiheit, die im Alltag durchaus gegeben ist, gelingt es deshalb eher, das Blickfeld und dessen Veränderung zu erfassen, als die Messung des Blickverlaufs. 
Bisherige Blickverlaufsstudien mit POS-Bezug sind bislang i.d.R. im Labor durchgeführt worden. Chandon et al. (2001, 2002) sowie Young (2002), der über das Vorgehen des Unternehmens „Perception Research Services“ berichtet, gehen zwar in die Geschäfte, arbeiten dort aber nicht am Regal, sondern in laborähnlichen Einrichtungen vor Ort. Dort zeigen sie den Besuchern Fotos von Produkten bzw. Marken anhand von Diaprojektionen, um dabei visuelle Zuwendungsreaktionen per Blickregistrierung zu erfassen und Kaufbereitschaften abzufragen. Gemessen wird dabei die Zeit bis zur ersten Blickfixierung, die Anzahl der Fixierungen, die Dauer der Fixierung bestimmter Flächen (gazes) und die gesamte Betrachtungsdauer - jeweils bezogen auf ein Produkt bzw. eine Marke. Visuelle Regalzuwendungen hat auch Leven (1992) anhand von im Labor gezeigten Regaldias untersucht. Pieters \& Warlop (1999) arbeiteten ebenfalls mit Regaldias. Schröder \& Berghaus (2005) setzten dagegen ein nachgebautes, reales Süßgebäckregal ein. Leven (1992) interessierte sich für die Trefferquote bei der Suche bestimmter Zeitschriftentitel und die „Struktur“ der Suche (S. 16f.). Pieters \& Warlop (1999) bestimmten sog. intra-brand saccades and inter-brand saccades (S. 11), mussten aber zehn Probanden wegen Kalibrierungsproblemen aussondern. Schröder \& Berghaus (2005) ließen drei Artikel, deren Kauf für die Probanden in Frage kam, suchen, um dabei den Blickverlauf aufzuzeichnen. Die Videoaufzeichnung gelang, nicht aber die geplante, rechnergestützte Erfassung des Blickverlaufs, so dass die Videos per Hand (per „manuelle Transkription“) ausgewertet werden mussten (S. 322). Immerhin konnte auf diese Weise in Erfahrung gebracht werden, wie viele Fixationen auf bestimmte Flächenkategorien entfielen: erstens auf die Regalflächen, zweitens auf die entnommenen Produkte und drittens auf sonstige Dinge (S. 322f.).

$\mathrm{Zu}$ den seltenen Blickverlaufsstudien am Regal, die im Feld durchgeführt wurden, zählt die Untersuchung von Berghaus (2005) (s. auch Schröder et al. 2005). Diese Studie befasste sich in drei Warenhäusern mit dem Blickverhalten in der Warengruppe Frischkäse, und zwar mit der Häufigkeit und Dauer der 
Wahrnehmung bestimmter Regalflächen und mit dem Blickverlauf bzw. dem Blickverlaufsmuster (Berghaus 2005 S. 162f.). Dabei wurden solche Besucher in die Untersuchung einbezogen, die vorhatten, Frischkäse zu kaufen. Um aus den Aufzeichnungen verlässliche Anhaltspunkte gewinnen zu können, musste die Laufrichtung und der Abstand zum Regal - ein Meter - vorgegeben werden (S. 198). Dennoch: Es konnte nicht verhindert werden, dass die Testpersonen diesen Abstand teilweise über- oder unterschritten (ebd). Die rechnergestützte Erfassung der Blickverläufe misslang auch in dieser Studie, so dass die Blickfeldvideos auch hier „per Hand“ ausgewertet werden mussten (ebd S. 163f.). „Offensichtliche Fehler" konnten dabei, so die Autorin (S. 199), „durch sorgfältige manuelle Auswertung“ beseitigt werden.

\section{(5) Aufzeichnungen einer „Blickfeldkamera“}

Wenn immer die Aufzeichnung von Blickfixierungen und Blickverläufen auf Widerstände stößt, aber die Ausrichtung des Blickfeldes erhoben werden soll, liegt der alleinige Einsatz einer „Blickfeldkamera“ nahe. Hier bietet die moderne Technik interessante Möglichkeiten, so z.B. die Montage des kleinen unscheinbaren Objektivs auf oder in einer Baseballmütze (Belk \& Kozinets 2005 S. 131), das mit dem Rekorder am Gürtel oder in der Jackentasche verbunden ist. Dabei kommt es darauf an, dass die Kalibrierung der Blickfeldaufzeichnung durchgeführt und noch während des Geschäftsbesuchs immer wieder überprüft wird.

\section{Beurteilung der Videoaufnahmen bzw. der Videographie}

Das große Potenzial von Videoaufnahmen ergibt sich aus der Tatsache, dass sie Abläufe recht realitätsnah wiedergeben, neben Bildern auch Töne bzw. Geräusche festhalten, Hinweise auf die Verhaltenssituation mitliefern und sich anschließend beliebig oft und damit mit immer besseren Einsichten auswerten lassen, sei es vom Forscher alleine, sei es unter Hinzuziehung der Probanden und deren 
Kommentare. Belk \& Kozinets (2005 S. 137) sprechen deshalb von einem „story telling potential“" der Videographie.

Die einzelnen Optionen der Videographie sind recht unterschiedlich zu beurteilen. Bereits die Anforderungen an die Technik variieren beachtlich. Digitale Überwachungskameras mögen leicht zu erwerben und zu montieren sein, schwierig ist dagegen das Zusammenführen der Aufnahmen im Rahmen eines Kundentracking sowie die automatische Anonymisierung aller aufgenommenen Personen. Beim begleitenden Kameramann bestehen enorme Herausforderungen darin, das Verhalten richtig und aussagekräftig zu erfassen und danach das digitale Video zum sofortigen Abspielen auf einem ausreichend großen Bildschirm zu bringen und während des Abspielens die Möglichkeit zum parallelen Aufzeichnen der Kommentierung so zu schaffen, dass das Kundenvideo und die Bezugspunkte in den Kommentaren für spätere Auswertungen einander zuordenbar sind. Regalgerichtete Kameras sollen unauffällig und zudem so installiert werden, dass ein Blick auf alle Zuwendungsreaktionen gelingt. Hohen Aufwand verursacht der Kameraeinsatz hinter einem Regal insbesondere dann, wenn dieser unauffällig und dennoch effektiv sein soll. Allergrößte Schwierigkeiten sind mit dem Versuch verbunden, mit Augenkameraaufzeichnungen den Fokus und den Verlauf der visuellen Zuwendung im Laden zu erfassen. Mehrere Versuche in dieser Richtung sind fehlgeschlagen. Im Vergleich dazu stehen die Chancen einer technischen Realisierung der Blickfeldregistrierung deutlich besser.

Die soziale Akzeptanz der Videographie stößt sicher auf Grenzen. Sicherheitskameras sind für die Erfassung von Diebstählen installiert und dürfen nicht umfunktioniert werden. Selbst wenn neue Systeme den Bedenken der Juristen Rechnung tragen würden, wäre zu befürchten, dass die Kunden solche Systeme dennoch ablehnen. Eine negative Privatkommunikation würde jenen Konkurrenten in die Hände spielen, die auf entsprechende Aufzeichnungen verzichten. Allerdings kann eine informierte und dezidierte Einwilligung von Kunden und Mitarbeitern in speziellen Versuchsläden nicht ausgeschlossen werden. 
Die interne Validität von Videoaufnahmen hängt im Wesentlichen von der Zuordenbarkeit von Aufnahmen und Personen sowie von der Kalibrierung ab. Je höher die Ansprüche an die Vollständigkeit und Genauigkeit ausfallen, desto leichter wird es sein, die Aussagekraft der Videographie in Zweifel zu ziehen. Optimismus ist insbesondere dann angebracht, wenn lediglich Kundenlaufmuster und die Zuwendung zu relativ großen Flächen, z.B. Produktgruppen, erhoben werden sollen. Auch wird es weiterhin leichter sein, die Validität von Zuwendungsanalysen im Labor und in laborähnlichen Settings zu sichern als in alltäglichen Einkaufsumgebungen (Belk \& Kozinets 2005 sprechen hier von „naturalistic observations"). Entsprechend differenziert müssen die Urteile bezüglich der externen Validität von Videoaufzeichnungen ausfallen. Geringe Bereitschaften, sich von einem Kameramann begleiten zu lassen (in unseren Studien etwa 20\%), eine Augenkamera oder eine „aufgerüstete Baseballmütze“ zu tragen, können nicht überraschen. Auch die Reaktionen der Probanden auf solche Messungen tragen zur Begrenzung der externen Validität bei. Allerdings gilt auch: mit dem Verzicht auf Generalisierungsmöglichkeiten (Einschränkung der externen Validität) lässt sich immerhin eine veritable Steigerung der Aussagekraft (Steigerung der internen Validität) erreichen.

\subsection{Registriertechniken}

Unter einer Registrierung sei hier das Erfassen von Sachverhalten verstanden, bei der kein Personal zum Einsatz kommt. Genauer betrachtet gilt dies auch für bestimmte Videotechniken, z.B. für die Aufzeichnungen eines Überwachungs- bzw. CCTV-Systems oder einer fest installierten Regalkamera, so dass die hier gewählte Einteilung in Videographie und Registrierung nicht ganz überschneidungsfrei sein kann.

Registrierungstechniken lassen sich auch am POS einsetzen. Dabei ist zunächst an Kontaktzähler an bestimmten Punkten zu denken, z.B. an die Erfassung des Besucheraufkommens anhand von Lichtschranken und an die Kontaktmessung im Fußboden. Für die Erfassung des Kundenlaufs ist aber auch an 
Registrierungssysteme zu denken, mit denen das Verhalten auf individueller Ebene erfasst werden kann. Der computergestützte Einsatz von Raumkameras ist bereits angesprochen worden. Denkbar und machbar sind darüber hinaus sog. Ortungssysteme. Über Kommunikationsnetze wie Mobilfunk und RFID können Einkaufswagen, Einkaufskörbe und ggf. auch Personen geortet und somit verfolgt werden, wenn sie entsprechend ausgerüstet worden sind. RFID-Tags, die auf Funksignale ansprechen und ID-Daten bereit halten, können in Einkaufskörben und Einkaufswagen, aber auch in Kundenkarten oder Bonusbelege eingebaut werden und den ablesenden Sendern den Standort des Besuchers signalisieren. Denkbar sind aber auch Tags in den Regalen und in anderen Präsentationsflächen, die auf Funksignale eines Einkaufswagens, eines Einkaufskorbes oder einer Kundenkarten reagieren und deren Kennung vom mobilen Sender aufgezeichnet wird.

Autoren wie Sorensen (2003) und Larson, Bradlow \& Faden (2005) denken in erster Linie an das Tracking von Einkaufswagen. Larson et al. (2005) sehen in der Bewegung von Einkaufswagen eine gute Annäherung in das Laufverhalten der Besucher, weil sich diese in der Bewegungsphase in unmittelbarer Nähe des Wagens befinden und sich in der Stopphase in seiner Nachbarschaft aufhalten (S. 396). Eine Ortung von Personen rückt ebenfalls in greifbare Nähe, so dass sich der Rückschluss vom Wagenlauf auf den Kundenlauf überprüfen ließe. Und wenn RFID-Tags in Kundenkarten eingebaut sind, lassen sich Verhaltensmuster bei wiederholten Ladenbesuchern identifizieren. Kann freiwilligen Ladenbesuchern der RFID-Tag als Bestandteil eines Gutscheins übergeben werden, der nach dem Ladenbesuch z. B. an der Kasse eingelöst werden darf, wäre eine Personenidentifizierung nicht erforderlich.

\section{Beurteilung der Registriertechniken}

Auch wenn Ortungssysteme mit Tracking-Aufgaben heute als technisch realisierbar gelten, so verursachen sie doch hohe Anfangsinvestitionen und auch einen 
gewissen Wartungsaufwand. Das enorme Aufkommen an Datenmengen lässt sich über Stichprobenprogramme reduzieren und ein Information Overload über geeignete Datenauswertungsprogramme vermeiden. Die soziale Akzeptanz könnte durch die Warnung vor dem gläsernen Konsumenten beeinträchtigt werden, und die Diskussion dieses Themas ist bereits in vollem Gange. Dabei sollte es allerdings einen großen Unterschied machen, ob personenbezogene, zurechenbare Daten oder personenbezogene, aber nicht zurechenbare Daten erhoben und gespeichert werden. Jedenfalls ist die Gefahr zu beachten, dass über die Zeitschiene Warenkorbdaten im Scannersystem und Trackingdaten zusammengeführt werden können, so dass sich anhand einer Datenfusion die Anonymisierung unterlaufen lässt.

Ein großer Vorteil der Registrierung ist in der internen sowie externen Validität zu sehen. Die Messungen sind zuverlässig und nicht reaktiv, das offene Personentracking und die Reaktion bestimmter Kunden auf die Trackingdiskussion in den Medien ausgenommen. Eine Einschränkung ist auch dort zu machen, wo vom Wagenlauf auf den Kundenlauf geschlossen wird. Noch liegen keine belastbaren Hinweise vor, die einen solchen Schluss rechtfertigen. Und zur externen Validität ist festzuhalten, dass die Registrierung durch anonyme Ortungssysteme Vollerhebungen in realitätsnahen Verhaltenssituationen ohne weiteres erlaubt.

\subsection{Befragungsmethoden}

Das Verhalten ist einer Selbstbeobachtung grundsätzlich zugänglich und somit auch einer Befragung. Dabei bedarf es jedoch einer beachtlichen Selbstaufmerksamkeit und der Bereitschaft, das eigene Verhalten anderen korrekt mitzuteilen. Liegt das zu erfragende Verhalten längere Zeit zurück, so muss es auch erinnert werden. Dasselbe gilt dort, wo es sich um einen recht lange andauernden Ladenbesuch handelt, und dort, wo zahlreiche und vielfältige Zuwendungsreaktionen erfasst werden sollen. Betrachten wir nun einige Befragungsvarianten 
und teilen wir diese nach dem zeitlichen Abstand zum Geschehen in eine spätere Befragung, eine Sofort-Danach-Befragung und eine zeitgleiche Befragung ein.

\section{(1) Die spätere Befragung}

Befragungen zum POS-Verhalten, die im Nachhinein und vermutlich an anderem Ort stattfinden müssen, stoßen schnell auf Grenzen. Sie können allerdings geeignet sein, grobe Anhaltspunkte zum Laufverhalten zu liefern und Berichte über wichtige Zuwendungsreaktionen wie z.B. über das Auffinden eines lange gesuchten Produkts, über den impulsiven Kauf eines Sonderangebotes, über das Gespräch mit einem Verkäufer oder einer Servicekraft und über den Umtausch eines fehlerhaften Produkts. Haben mehrere Personen eingekauft, können die Angaben verschiedener Akteure miteinander verglichen werden.

\section{(2) Die Sofort-Danach-Befragung}

Eine Befragung des Ladenbesuchers, die sich an den Ladenbesuch unmittelbar anschließt, verspricht mehr Erfolg als eine spätere Befragung, denn das Erinnerungsvermögen wird nicht so stark in Anspruch genommen. Dies gilt in besonderem Masse für kurze Shoppingtrips und für den Besuch einer bestimmten Abteilung. Handelt sich dagegen um längere Besuche, bei denen zahlreiche Verhaltensakte zu berichten wären, stößt auch die Sofort-Danach-Befragung auf ihre Grenzen. Dann müssen ggf. verfügbare Erinnerungshilfen zum Einsatz kommen. Neben verbalen Hinweisen oder Skizzen, die den Verhaltensprozess und die jeweilige Umwelt in Erinnerung rufen, z.B. den Kundenlauf, kämen aber auch Videoaufnahmen in Frage, wenn es darum geht, konkrete Zuwendungsreaktionen in Erfahrung zu bringen. Erste Erfahrungen, die wir mit diesem Vorgehen sammeln konnten, zeigen recht gute Erfolge. 


\section{(3) Die zeitgleiche Befragung}

Wann immer mit einem geringen Involvement und/oder geringen Erinnerungsleistungen gerechnet werden muss, bietet sich auch eine Just-in-TimeBefragung an. Hierbei wird der Kunde gebeten, sein Verhalten schon während dessen Ausführung mitzuteilen. Die Mitteilungen lassen sich mit Hilfe eines Diktiergeräts aufzeichnen. Auch kann ein Begleiter die aktuellen Mitteilungen protokollieren. Der Einsatz eines Diktiergerätes hat auf den ersten Blick den Vorteil, dass auf das Mitlaufen eines Begleiters verzichtet werden kann. Doch besteht für diesen Fall die Gefahr, dass der Proband die erbetenen Just-in-TimeMitteilungen als belastend empfindet und deshalb vernachlässigt oder die erhaltene Verbalisierungsaufgabe ganz vergisst.

Die POS-Forschung kennt nur wenige Versuche, das Verhalten über die „Handle-laut-Methode“ zu erfassen. Bei Payne \& Ragsdale (1978) hatten Mütter bei mehreren wöchentlichen Einkaufstrips einer jungen Begleiterin gegenüber zu artikulieren, wohin sie gerade gehen, was sie gerade machen, was sie suchen und welche Produkte bzw. Marken sie gerade bemerken oder betrachten (S. 572). Zu dieser Aufgabe kam erschwerend hinzu, dass die Frauen auch zu berichten hatten, was ihnen jeweils durch den Kopf geht (ebd). Sie hatten also zweierlei zu konstatieren und zu formulieren: ihr Denken und ihr Handeln! Jedenfalls mussten die eingesetzten Begleitpersonen darauf achten, dass das laute Denken und Handeln der Ladenbesucher nicht abreißt.

Ähnlich groß war die Aufgabe bei Titus \& Everett (1996). Die Probanden sollten den Laden nach den Produkten, die auf einem Einkaufszettel aufgelistet waren, durchsuchen und dabei aussprechen, wo sie suchen, warum sie dort suchen, welche Anhaltspunkte sie nutzen, um sich im Laden zurechtzufinden, und was ihnen bei alledem sonst noch durch den Kopf geht (S. 267). Auch hier wurde eine Begleitperson mit auf den Weg gegeben, damit sie die Artikulation der Gedanken und des Verhaltens - wann immer nötig - in Gang hält (ebd). 


\section{Beurteilung der Befragungsmethoden}

Generelle Möglichkeiten und Grenzen der Befragungsmethode sind bekannt. Der Hinweis auf Grenzen der Selbstwahrnehmung, der Erinnerung, der Formulierung von Aussagen und der Bereitschaft, sich mitzuteilen, soll hier genügen. Wir können festhalten, dass die Machbarkeit von Befragungen und der Einsatz technischer Geräte zumeist keine Probleme aufwirft. Es ist zudem offenkundig, dass die soziale Akzeptanz einer Befragung im Laden darunter leiden kann, dass keine Zeit zur Verfügung steht, dass eine Begleitung stört und dass der Einblick in persönliche Dinge nicht erwünscht ist. Deshalb ist immer auch die interne Validität einer Befragung gefährdet. Dies gilt auch für die externe Validität, denn nicht jeder Ladenbesucher ist zu einer Befragung bereit und nicht jeder möchte beim Ladenbesuch begleitet werden (Selektivität). Außerdem können die zeitgleiche Befragung, vor allem die Begleitung, und die Ankündigung einer (Danach-) Befragung die Situation und damit das Verhalten der Probanden beeinflussen (Reaktivität).

\section{Auswählte Möglichkeiten der Methodenkombination}

Die Kombination verschiedener Messmethoden kann unterschiedliche Zwecke haben: zum einen die Überprüfung von Methoden, wenn sie vorgeben, dasselbe zu messen (Validierungsziel), zum anderen die Steigerung des Erkenntnispotentials, wenn die Methoden unterschiedliches messen und sich somit ergänzen (Synergieziel). Diese Kombination im Dienst einer Arbeitsteilung oder einer Zusammenarbeit soll hier nun im Vordergrund stehen. Beginnen wir mit der Methodenkombination innerhalb einer Methodengruppe, um dann gruppenübergreifende Kombinationen anzusprechen.

\section{Zur Methodenkombination innerhalb einer Methodengruppe}

(1) Kombination im Beobachtungsmix

Ein Beispiel für sinnvolle Beobachtungskombinationen liefert jener Fall, in dem der Forscher ausgewählte Ladenbesucher in ihrem Laufverhalten passiv, ggf. auch 
verdeckt beobachtet, während Mitarbeiter des Hauses an den Selbstbedienungsund Servicetheken dieselben Kunden ebenfalls verdeckt, aber teilnehmend, d.h. ihren üblichen Job ausführend, beobachten.

\section{(2) Kombination im Videomix}

Während ein Video-Überwachungs-System Kundenläufe erfasst und Laufmuster identifiziert, wird das konkrete Zuwendungsverhalten an bestimmten Regalen über festinstallierte Regalkameras aufgenommen. Denkbar wäre auch, dass das Verhalten am Regal mit zwei Kameras erfasst wird, indem die eine Kamera den Kunden von hinten aufnimmt, und die andere hinter dem Regal und hinter einem Einwegspiegel aufgestellt ist, um die visuelle Zuwendung zu erfassen.

\section{(3) Kombination im Mix der Registriermethoden}

Ein grobmaschiges Ortungssystem erfasst die Kundenläufe und Laufmuster, z.B. im Einkaufszentrum, während ein engmaschiges RFID-System die etwas genauere Erfassung des Lauf- und Zuwendungsverhalten an besonders interessanten Punkten, z.B. vor einem Regal oder bei einer Zweitplatzierung, erfasst und damit die grobmaschige Ortung ergänzt. Denkbar wäre auch eine Kombinationen der Personenortung und der Ortung des Einkaufswagens. Auf diese Weise ließe sich analysieren, wann und wo sich Wagen und Kunde trennen, wann sie sich wieder treffen, wie lange die Trennung dauert und wohin die betreffende Person in dieser Zeit gelaufen ist.

\section{(4) Kombination im Befragungsmix}

Interessiert der Weg, den ein Kunde gegangen ist, bis er ein bestimmtes Regal erreicht hat, und das Verhalten an diesem Regal, dann könnte folgende Methodenkombination in Betracht gezogen werden: offene oder gestützte Befragung zum bisherigen Laufweg und zeitgleiche Befragung bzw. Handle-lautMethode vor dem Regal. 


\section{Zur Methodenkombination über Methodengruppen hinweg}

Bei der Kombination von Methoden der Beobachtung, der Videographie, der Registrierung und der Befragung sind ebenfalls viele Möglichkeiten denkbar. Hier soll es genügen, wenn wir nur einige Kombinationsmöglichkeiten ansprechen.

\section{(1) Beobachtung und Befragung}

Eine Beobachtung des Verhaltens in einem bestimmten Ladenareal lässt sich verbinden mit der Befragung zum Weg, der eingeschlagen wurde, um zu dem betreffenden Areal zu gelangen. Eine Kombination von Beobachtung und Befragung findet sich auch beim „Shopping with the Consumer“" (Otnes et al. 1995, Lowrey et al. 2005). Die Begleitperson beobachtet das Verhalten im Laden, erfasst mit der Vorabbefragung die Besuchsmotive, ggf. auch die Kaufpläne, und mit der Nachherbefragung die ungeplanten Käufe sowie deren Begründung.

\section{(2) Beobachtung und Videographie}

Eine Kombination der Beobachtungs- und Videomethode könnte darauf abstellen, dass die Beobachter den Kundenlauf und die Mitnahme von Produkten erfassen, während die visuelle Zuwendung an den Regalen über die in den Regalen eingebauten oder hinter den Regalen aufgestellten Kameras erfasst wird.

\section{(3) Videographie und Registrierung}

Videographie und Registrierung können dergestalt verbunden werden, dass Lichtschranken vor einem Regal die Registrierung von Besuchern oder Betrachtern übernehmen, und deren Verhalten am Regal dann von einer Regalkamera übernommen wird.

\section{(4) Videographie und Befragung}

Eine Kombination von Videographie und Befragung liegt dort vor, wo ein Kameramann den Kundenlauf aufzeichnet und diese Aufzeichnung anschließend 
dazu dient, per videogestützter Erinnerung das Zuwendungsverhalten näher zu bestimmen. Ähnlich liegt der Fall dort, wo ein Videosystem den Kundenlauf festhält und das Zuwendungsverhalten an bestimmten Regalen anschließend erfragt wird.

\section{Zur Realisierung der Methodenkombination}

Die Kombination diverser Methoden kann in die Hände verschiedener Personen gelegt, kann aber auch von einer einzigen Person bewerkstelligt werden. In einer Studie zum Kundenverhalten in der Weihnachtsabteilung eines Verbrauchermarktes waren z.B. zwei Personen im Einsatz: die eine übernahm die verdeckte Beobachtung, die andere die sich daran anschließende Befragung. Gleich mehrere Aufgaben übernahm ein und dieselbe Person in der Untersuchung von Iyer (1989): Sie fuhr den Kunden zum Geschäft seiner Wahl und von dort auch wieder nach Hause. Außerdem hatte sie den Fahrgast zu Beginn nach seinen Kaufplänen zu fragen und am Ende die gekauften Produkte zu registrieren. Und während des Einkaufs musste diese Person dem Ladenbesucher auch noch unauffällig folgen und ihn beim Einkaufen ebenso unauffällig beobachten (S. 46f.), so dass sie insgesamt recht gut beschäftigt war.

\section{Die Sequenzanalyse als Datenauswertungsmethode}

Wenn es darum geht, das Verhalten am POS adäquat zu beschreiben, kommt es nicht nur auf die Validität der Datenerhebung an, sondern auch auf die Zweckmäßigkeit der Datenauswertung. Der Kundenlauf und das Zuwendungsverhalten sind als Sequenzen festzuhalten bzw. darzustellen und auch als solche zu analysieren. Dabei liegt es nahe zu fragen, ob Gruppen existieren, die sich in ihrem Lauf- und Zuwendungsverhalten ähneln. Derartige Analysen sind bislang allerdings Mangelware geblieben. In letzter Zeit gab es immerhin erste Versuche, das Laufverhalten am POS über die Auswertung von Kontaktsequenzen mithilfe einer Sequenzclusterung zu analysieren: Ruiz et al. (2004 S. 335) und Larson et al. (2005 S. 397-412). 
Bei einer Sequenzclusterung, die auch in anderen Bereichen der Konsumentenund Marketingforschung eine relativ neue Datenauswertungsmethode darstellt, tauchen ganz spezifische Fragen auf, die beantwortet werden müssen. Zu diesen Fragen zählen vor allem folgende: Wie lassen sich Sequenzen mit unterschiedlicher Länge vergleichen? Wie sollen Ähnlichkeiten und Distanzen operationalisiert werden und welche Distanz- oder Ähnlichkeitsmaße kommen in Frage? Und wie kann eine Sequenzclusterung bzw. Suche nach Verhaltensmustern dem Umstand Rechnung tragen, dass sich Lauf- und Zuwendungssequenzen auf unterschiedliche Verhaltensebenen beziehen, aber dennoch gemeinsam berücksichtigt werden wollen?

Bei der Auswertung einer Kundenlaufsstudie in einem Supermarkt haben wir den Versuch unternommen, Sequenzcluster zu bestimmen. Die Abfolgen von Flächenkontakten dienten als Abbildung der Verhaltenssequenzen. (Ebenfalls erfasste Zuwendungsaktivitäten wurden dabei nicht berücksichtigt.) Die Distanz zwischen Lauf- bzw. Flächenkontaktsequenzen berechneten wir anhand des Levenshtein-Algorithmus (Levenshtein 1965), d.h. dass eine Distanz zwischen zwei Sequenzen umso größer ist, je mehr Operationen bei der Umwandlung der einen Sequenz in die andere Sequenz erforderlich sind.

Die Suche nach homogenen Sequenzclustern bzw. unterscheidbaren Verhaltensmustern (mithilfe der Ward-Methode) führte zu drei unterscheidbaren Gruppen. Eine Kurzbeschreibung dieser drei Lauf-Cluster läßt sich anhand der folgenden Zentroide vornehmen:

Cluster 1 (n=44): Frisches Obst \& Gemüse $>$ Backwaren $>$ Tiefkühlprodukte $>$ Food trocken \& verpackt $>$ Tiefkühlprodukte $>$ Kassenzone,

Cluster 2 (n=14): Frisches Obst \& Gemüse $>$ Backwaren $>$ Tiefkühlprodukte $>$ Food trocken \& verpackt $>$ Getränke $>$ Tiefkühlprodukte $>$ Kosmetika \& Hygieneartikel > Kassenzone, 
Cluster 3 ( $\mathrm{n}=22)$ : Food trocken \& verpackt $>$ Tiefkühlprodukte $>$ Backwaren $>$ Frisches Obst \& Gemüse $>$ Kassenzone

Um das mehr oder weniger homogene Laufverhalten dieser drei Cluster als Verhaltensmuster etwas anschaulicher darzustellen, wählten wir fünf Laufsequenzen aus, die im Vergleich zu den übrigen Laufsequenzen die geringsten Abstände zum Zentroid aufweisen. Diese „Zentroid-Proxies" lassen sich graphisch darstellen und sind in den Abbildungen 1 - 3 wiedergegeben. $\mathrm{Zu}$ dieser Ergebnisdarstellung sei angemerkt, dass es sich jeweils um eine vereinfachte, schematische Darstellung der Laufwege handelt.

\section{Aktuelle und künftige Trends der POS-Forschung}

Nachdem wichtige Möglichkeiten einer Verhaltensforschung am POS aufgezeigt worden sind, seien derzeit erkennbare und zu erwartende Trends in der POSForschung angesprochen. Zu den aktuellen Trends sind vor allem drei zu zählen. Erstens: Das Interesse am POS-Verhalten steigt. Dies gilt nicht nur in für die Praxis, sondern auch für die Wissenschaft, wie zunehmende Einladungen zu Beiträgen in Veröffentlichungen und zu Fachtagungen zeigen. Zweitens: Die Analyse des Kundenverhaltens am POS wird intensiviert. Nachdem die Analyse von Kundenreaktionen auf Maßnahmen des In-Store-Marketing immer schon durchgeführt wurden, nehmen jene Studien zu, die sich ganz gezielt für das von POS-Maßnahmen mehr oder weniger unabhängige Lauf- und Zuwendungsverhalten der Ladenbesucher interessieren. Die Analyse des Click- und Blickverhaltens im Internet hat dabei zumindest bei einigen Studien Pate gestanden. Drittens: Neuere Versuche einer POS-Verhaltensforschung erkennen zunehmend die Möglichkeiten, die sich aus der Nutzung digitaler Ortungs-, Aufnahme- und Präsentationstechnik ergeben. $\mathrm{Zu}$ nennen wäre hier vor allem der Mobilfunk, die RFID-Technik, die Videotechnik und die EDV auf installierten und mobilen Geräten. 
Abbildung 1. Zentroid-Proxies im Kundenlaufcluster $1(n=44)$

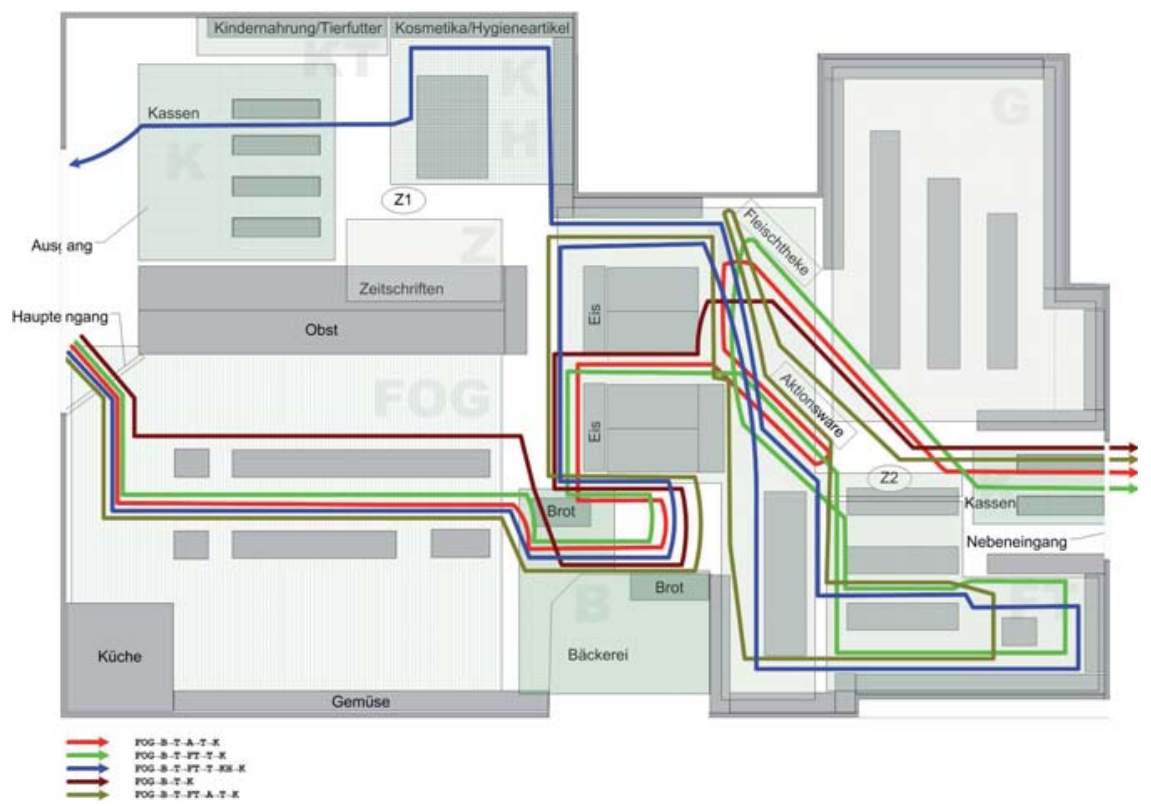

Abbildung 2. Zentroid-Proxies im Kundenlaufcluster $2(n=14)$

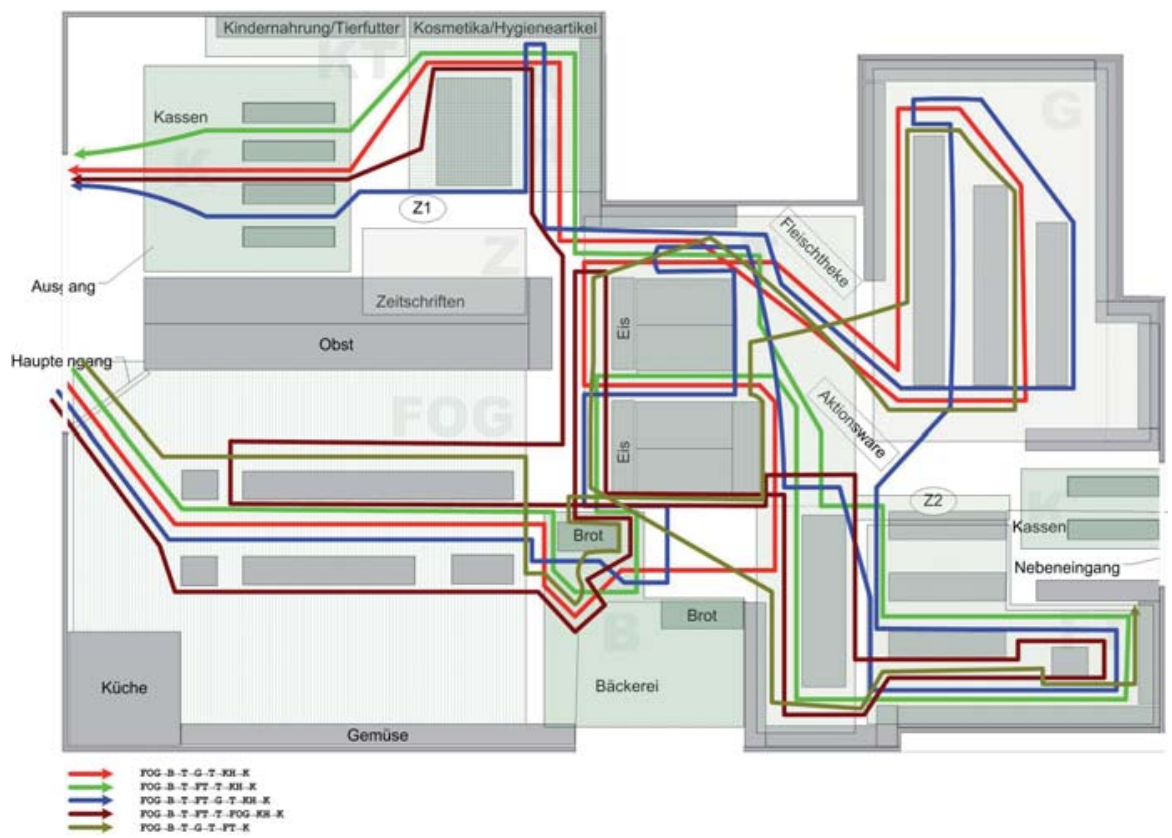


Abbildung 3. Zentroid-Proxies im Kundenlaufcluster $3(n=22)$

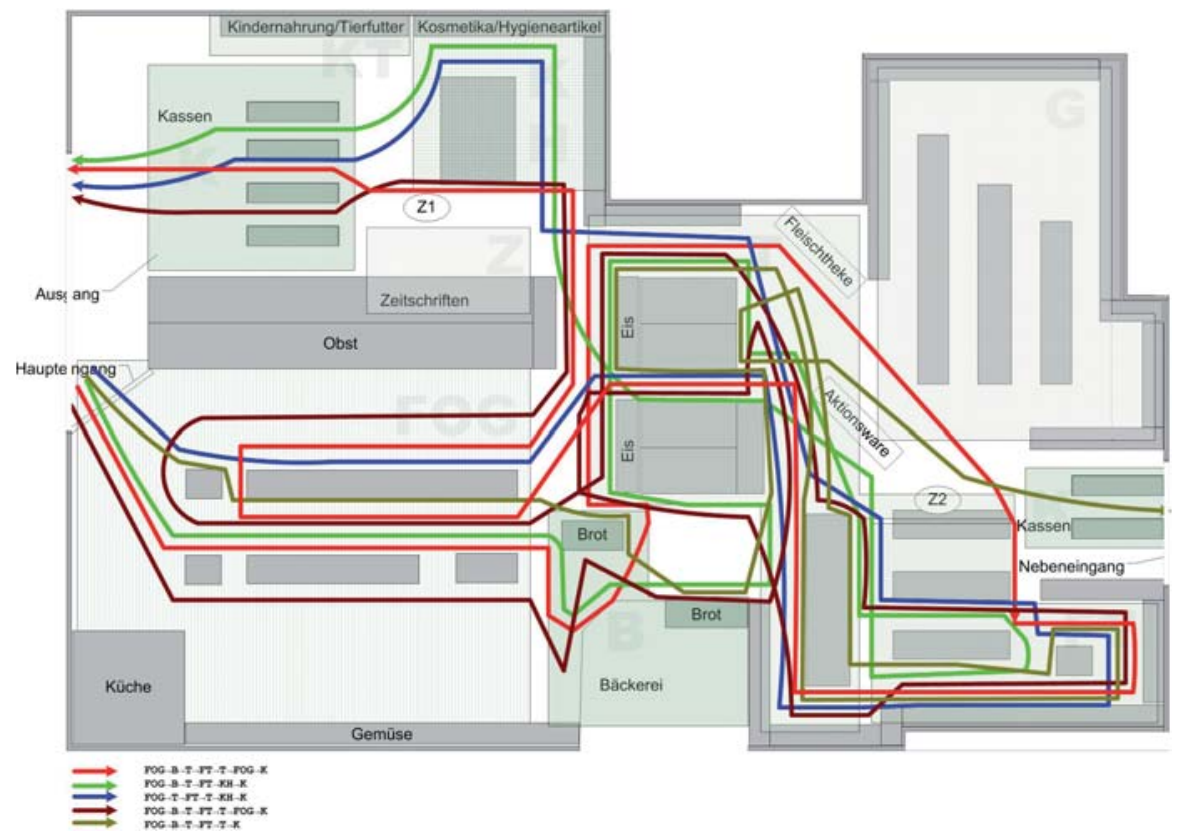

Und für die Zukunft sind darüber hinaus weitere Entwicklungen zu erwarten: Zu ihnen zählen die Erprobung bislang nicht eingesetzter Methoden und Methodenkombinationen, der Einsatz und die Weiterentwicklung angemessener Datenauswertungsverfahren, insbesondere der Sequenzanalyse und der Sequenzclusterung, und die Fusion von Datensätzen aus den Scannerkassen, aus Kundendatenbanken und aus gezielten Erhebungen zum POS-Verhalten und - damit eng verbunden - die verstärkte Diskussion der rechtlichen und ethischen Fragen, die mit einer Sammlung und Nutzung personenbezogener Daten zum POS-Verhalten verbunden sind. Schließlich ist auch zu erwarten, dass neben der Erfassung des POS-Verhaltens künftig verstärkte Versuche unternommen werden, dessen Ursachen zu bestimmen. Dies impliziert entsprechende Untersuchungsdesigns wie die Durchführung von Experimenten und Quasi-Experimenten (z.B. die gezielte Veränderung von Regalanordnungen und die Durchführung von In-Store- 
Promotions), aber auch den Rückgriff auf neuere Theorien zum situativen Denken, Fühlen und Handeln (vgl. dazu z.B. Smith \& Semin 2004).

\section{Herausforderungen an die wissenschaftliche POS-Forschung}

Während sich die unternehmenseigene und die kommerzielle Forschung in Zukunft vor allem für solche Analysen interessieren wird, deren Ergebnisse sich unmittelbar in zielführende Marketingmaßnahmen umsetzen lassen, kann und muss sich die akademische oder wissenschaftliche Forschung vor allem mit Grundfragen befassen. Zu diesen Grundfragen zählen bei der Entwicklung und Beurteilung eines Methodenarsenals zum einen die Beurteilung einzelner Erfassungsinstrumente und zum anderen die Beurteilung zielführender Methodenkombinationen. Dies bedeutet im einzelnen: (1) die technische Realisierbarkeit und den Aufwand an Zeit, Geld und Personal, aber auch zum Know-how, näher untersuchen, (2) die soziale Akzeptanz bestimmter Messverfahren bei Kunden, Mitarbeitern und Führungskräften, aber auch in der Gesellschaft (inklusive Gesetzgebung und Rechtsprechung), zu bestimmen, (3) die Validität der Methoden unter Berücksichtigung der jeweiligen Einsatzmodalitäten zu testen und (4) Aussagen zur ökologischen Validität zu formulieren, in denen die Selektivitätseffekte in den Stichproben und die Reaktivität im Verhalten der Probanden zur Sprache kommen. Von entscheidender Bedeutung muss das Streben nach interner Validität von Messungen des POS-Verhaltens sein. Auch wenn dies große Herausforderungen mit sich bringt, können wir uns der Validitätssicherung schrittweise nähern, indem wir mit der Augenscheinvalidität bzw. mit der Konsultation von Mess-Experten beginnen und zugleich die Suche nach messbaren Außenkriterien intensivieren und damit Tests der Kriteriumsvalidität vorbereiten und uns schließlich der nomologischen Validität zuwenden. Letzteres bedeutet, dass wir uns um eine Theorie bemühen, die Auskunft darüber gibt, welche Messinstrumente und welche Methodenkombination in welcher Anwendung richtige Auskünfte über das faktische POS-Verhalten liefern. 


\section{Literatur}

Babin, B. J. \& Darden, W R. (1995).

Consumer Self-Regulation in: A Retail Environment, in: Journal of Retailing, Vol. 71 (1995), No 1, pp. 47-70

Baldauf, A., Srnka, K. J. \& Wagner, U. (1997).

Untersuchung eines neuartigen Shop-Konzeptes mittels Kundenlaufstudie, in: der markt, 36. Jg. (1997), Heft 3, S.142-143, Heft 4, S. 103-111

Belk, R. W. \& Kozinets, R. V. (2005).

Videography in Marketing and Consumer Research, in: Qualitative Market Research: An International Journal, Vol. 8 (2005), No 2, pp. 128-141

Berghaus, N. (2005).

Eye-Tracking im stationären Einzelhandel. Eine empirische Analyse der Wahrnehmung von Kunden am Point of Purchase, Lohmar-Köln: Josef Eul Verlag

Bitner, M. J. (1992).

Servicescapes: The Impact of Physical Surroundings on Customers and Employees, in: Journal of Marketing, Vol. 56 (April 1992), pp. 57-71

Bloch, P. H. \& Richins, M. L. (1983).

Shopping Without Purchase: An Investigation of Consumer Browsing Behavior, in: Advances in Consumer Research, Vol. 10 (1983), pp. 389-393

Botelho, V. M. (2002).

Analyseverfahren zum Kundenlauf. Unveröffentlichte Diplomarbeit am Institut für Marketing und Handel der Universität Göttingen, Abteilung Marketing

Büttner, O. B., Rauch, M. \& Silberer, G. (2005).

Consumer Cognition at the Point of Sale: Results From a Process Tracing Study, Beiträge zur Trackingforschung, Nr. 11, Universität Göttingen: Institut für Marketing und Handel

Chandon, P., Hutchinson, W.J. \& Young, S. H. (2001).

Measuring the Value of Point-of-Purchase Marketing with Commercial EyeTracking Data, working paper 2001/19/MKT, INSEAD, Fontainebleau

Chandon, P., Hutchinson, J. W. \& Young, S. H. (2002).

Unseen is Unsold: Assessing Visual Equity with Commercial Eye-Tracking Data, working paper 2002/85/MKT, INSEAD, Fontainebleau 
De Heer, J., Groenland, E. \& Bloem, S. (2000).

To Be or Not To Be Included in the Consideration Set? The Effects of Brand Conspicuity on Exploratory Visual Search Behavior and Memory, in: J. Inman, K. Tepper \& T. Whittler (Eds.). Proceedings of the Society for Consumer Psychology Winter Conference, San Antonio, TX: Society for Consumer Psychology

Granbois, D. H. (1968).

Improving the Study of Customer In-store Behavior, in: Journal of Marketing, Vol. 32 (October 1968), pp. 28-33

Hicks, J. W. and Kohl, R. L. (1955).

Memomotion Study as a Method of Measuring Consumer Behaviour, in: Journal of Marketing, Vol. 20 (October 1955), pp. 168-170

Hoyer, W. D. (1984).

An Examination of Consumer Decision Making for a Common Repeat Purchase Product, in: Journal of Consumer Research, Vol. 11 (December 1984), pp. 822-829

ISB-Studie (1986).

Kundenlauf-Studie in einem SB-Warenhaus, in: BdSW - Bundesverband der Selbstbedienungs-Warenhäuser (Ed.). Köln: Institut für Selbstbedienung und Warenwirtschaft

Iyer, E. S. (1989).

Unplanned Purchasing: Knowledge of Shopping Environment and Time Pressure, in: Journal of Retailing, Vol. 65 (Spring 1989), No 1, pp. 40-57

Kahn, B. E. \& Schmittlein, D. C. (1992).

The Relationship Between Purchases Made on Promotion and Shopping Trip Behavior, in: Journal of Retailing, Vol. 68 (Fall 1992), No 3, pp. 294-315

Larson, J. S., Bradlow, E. T. \& Faden, P. S. (2005).

An Exploratory Look at Supermarket Shopping Paths, in: International Journal of Research in Marketing, Vol. 22 (2005), No 4, pp. 395-414

Leven, W. (1992).

Warenpräsentation im Einzelhandel, dargestellt am Beispiel der Zeitungs- und Zeitschriftenpräsentation, in: Marketing ZFP, 14.Jg. (1992), Heft 1, pp.13-22

Levenshtein, V. I. (1965).

Binary Codes Capable of Correcting Deletions, Insertions und Reversals, in: Doklady Akademi Nauk SSSR, Vol. 163 (1965), No 4, pp. 845-848 
Lowrey, T. M., Otnes, C. C. \& McGrath, M. A. (2005).

Shopping with Consumers: Reflections and Innovations, in: Qualitative Market Research: An International Journal, Vol. 8 (2005), No 2, pp. 176-188

Newman, A. J. \& Foxall, G. R. (2003).

In-store Customer Behaviour in the Fashion Sector: Some Emerging Methodological and Theoretical Directions, in: International Journal of Retail \& Distribution Management, Vol. 31 (2003), No 11, pp. 591-600

O’Neill, M. J. (1992).

Effects of Familiary and Plan Complexity on Way Finding in Simulated Buildings, in: Journal of Environmental Psychology, Vol. 12 (1992), No 4, pp. 319-327

Otnes, C. C., McGrath, M. A. \& Lowrey, T. M. (1995).

Shopping with Consumers: Usage as Past, Present and Future Research Technique, in: Journal of Retailing and Consumer Services, Vol. 2 (1995), No 2, pp. 97-110

Payne, J. W. \& Ragsdale, E. K. E. (1978).

Verbal Protocols and Direct Observation of Supermarket Shopping Behavior: Some Findings and a Discussion of Methods, in: H. Keith Hunt (Ed.). Advances in Consumer Research, Chicago, IL: Association for Consumer Research, Vol. 5 (1978), pp. 571-577

Pieters, R. \& Warlop, L. (1999).

Visual Attention During Brand Choice: The Impact of Time Pressure and Task Motivation, in: International Journal of Research in Marketing, Vol. 16 (1999), No 1, pp. 1-16

Ruiz, J.-P., Chebat, J.-C. \& Hansen, P. (2004).

Another Trip to the Mall: A Segmentation Study of Customers Based on Their Activities, in: Journal of Retailing and Consumer Services, Vol. 11 (2004), pp. 333 350

Russo, J. E.\& Leclerc, F. (1994).

An Eye-Fixation Analysis of Choice Processes for Consumer Nondurables, in: Journal of Consumer Research, Vol. 21 (Sept. 1994), pp. 274-290

Schröder, H. \& Berghaus, N. (2005).

Blickaufzeichnung der Wahrnehmung am Regal - Methodendemonstration am Beispiel Süßgebäck, in: Trommsdorff, V. (Hrsg.). Handelsforschung 2005. Neue Erkenntnisse für Praxis und Wissenschaft des Handels, Stuttgart: Kohlhammer, S. 315-335 
Schröder, H., Berghaus, N. \& Zimmermann, G. (2005).

Das Blickverhalten der Kunden als Grundlage für die Warenplatzierung im Lebensmitteleinzelhandel, in: der markt, Vol. 44 (2005), No 172, pp. 31-43

Silberer, G. (2005).

Die videogestützte Gedankenrekonstruktion kognitiver Prozesse beim Ladenbesuch, in: Marketing ZFP, 27. Jg. (2005), Heft 4, S. 263-271

Smith, E. R. \& Semin, G. R. (2004).

Socially Situated Cognition: Cognition in its Social Context, in: Advances in Experimental Social Psychology, Vol. 36 (2004), pp. 53-117

Sorensen, H. (2003).

The Science of Shopping, in: Marketing Research, Vol. 15 (2003), No 3, pp. 30-53

Titus, P. A., \& Everett, P. B. (1996).

Consumer Wayfinding Tasks, Strategies and Errors: An Exploratory Field Study, in: Psychology \& Marketing, Vol. 13 (1996), No 3, pp. 265-290

Weismann, J. (1981).

Evaluating Architectural Legibility, in: Environment and Behavior, Vol. 13 (1981),

No 2, pp. 189-204

Wells, W. D. \& LoSciuto, L. A. (1966).

Direct Observation of Purchasing Behavior, in: Journal of Marketing Research, Vol. 3 (August 1966), pp. 227-233

Young, S. (2002).

Winning at Retail, in: Global Cosmetic Industry (GCI), Vol. 10 (Aug. 2002), pp. 61-64

Zielke, S. (2002).

Kundenorientierte Warenplatzierung, Stuttgart - Berlin - Köln: Kohlhammer 

C

Die Erfassung kognitiver Prozesse beim Ladenbesuch 


\section{Einleitung}

Das Lauf- und Zuwendungsverhalten am POS entscheidet über die Anzahl und die Qualität der Kontakte mit dem Angebot. Über das Kaufverhalten entscheidet aber auch das, was dem Besucher durch den Kopf geht - also der Gedankengang bzw. die kognitiven Prozesse. $\mathrm{Zu}$ diesen Prozessen zählen Überlegungen zur Realisierung von Kaufplänen, Überlegungen zu ungeplanten Kaufmöglichkeiten, Überlegungen bei der Suche von Produkten und bei der Orientierung im Laden, Überlegungen in Bezug auf Serviceangebote, auf das Verhalten von Mitarbeitern, auf das Verhalten der Kaufbegleitung sowie Überlegungen, die mit dem Geschäft überhaupt nichts zu tun haben, z.B. Gedanken, die den weiteren Tagesverlauf betreffen - kauf- und konsumbezogene Überlegungen ausgenommen.

Die Erfassung kognitiver Prozesse beim Ladenbesuch, auf die wir uns hier konzentrieren wollen, ist mit besonderen Herausforderungen verbunden, da es sich um ein verdecktes Verhalten - ein „,covert behavior“ - handelt. Wie diesen Herausforderungen durch die Wahl geeigneter Erfassungsmethoden begegnet werden kann, soll uns hier interessieren und beschäftigen. Dabei sollen nicht nur die klassischen Methoden zur Sprache kommen, sondern auch Möglichkeiten, das relevante Methodenarsenal im Blick auf neue Hilfsmittel, die der technische Fortschritt mit sich gebracht hat, zu erweitern bzw. zu ergänzen.

Wenn im Folgenden allein die Möglichkeiten und Probleme einer Erfassung kognitiver Prozesse beim Ladenbesuch zur Sprache kommen, wollen wir damit kein Plädoyer für eine rein deskriptive Erfassung kognitiver Prozesse am POS abgeben - ganz im Gegenteil: In der aussagekräftigen Erfassung kognitiver Prozesse am POS sehen wir die Ansatzpunkte und Anlässe für eine erweiterte Theorie des Konsumentenverhaltens, für eine Theorie, die es im Anschluss an neue Erkenntnisse bezüglich der kognitiven Prozesse am POS zu entwickeln gilt und die dann besser als klassische Erklärungsversuche in der Lage sein sollte, das konkrete Verhalten und Erleben von Kunden bzw. Besuchern am POS zu erklären und zu prognostizieren. 
Aber selbst deskriptive Befunde können von großer Bedeutung sein. $\mathrm{Zu}$ denken wäre hier vor allem an die oft zitierte Aussage, der zufolge die meisten Kaufentscheidungen der Verbraucher beim Kauf alltäglicher Dinge erst im Ladengeschäft - also vor Ort - getroffen werden. Eine Überprüfung dieser Aussage, aber auch deren Präzisierung, setzen voraus, dass es gelingt, die Kaufentscheidungen und damit die Überlegungen der Ladenbesucher richtig zu erfassen. Die Erfassung von Gedanken von Ladenbesuchern interessiert jedenfalls auch vor diesem Hintergrund, zumal Aussagen zur relativen Häufigkeit von Kaufentscheidungen am POS für das Marketing der Händler, aber auch für das Marketing der Hersteller von allergrößter Bedeutung sind.

\section{Mögliche Überlegungen eines Ladenbesuchers}

Bevor wir auf die Möglichkeiten einer Erfassung kognitiver Prozesse bei Ladenbesuchern eingehen, soll zunächst die Vielfalt möglicher Überlegungen vor Ort zumindest grob skizziert werden.

Beim Betreten eines Ladens können zunächst die Kaufabsichten und/oder die kürzlich erhaltenen Hinweise auf Angebote und Preise im Vordergrund der Überlegungen stehen. Bei ernsthaften Kaufabsichten und hohem Zeitdruck werden die Orientierung im Geschäft, die Suche der Ware vor Ort und die Auswahl von Marken und Artikeln am Regal bzw. an der Bedienungstheke dominieren. Der sog. Seeker geht gezielt vor und fokussiert seine Überlegungen auf das Auffinden des relevanten Standortes und die richtige Auswahl vor Ort.

Demgegenüber will sich der Browser bzw. der Surfer nur mal umschauen, sich informieren oder sich anregen lassen. Dann dominiert die Wahrnehmung und meist recht kurze Betrachtung des Angebots - sei es das gesamte Sortiment, sei es ein Sortimentsteil. Er ist vielleicht bereit, ungeplante Käufe zu tätigen, wenn sich etwas Interessantes oder etwas Günstiges findet. Es dominiert eine Art Reizoffenheit und die Gedanken versteifen sich nicht darauf, geplante Käufe möglichst effizient zu realisieren. 
Während eines Ladenbesuches können sich die Absichten bzw. die Motive sowie die Ausrichtung des Verhaltens ändern. Ein Seeker kann seine Pläne hintenan stellen, verschieben oder gar vergessen und in den Surfer-Modus wechseln. Dies ist auch möglich, nachdem er seine Kaufziele erreicht, die beabsichtigten Käufe realisiert hat. Der Surfer kann ebenfalls dazu übergehen, Kaufinteresse und Kaufabsichten zu entwickeln und Produkte nicht nur zu finden, sondern auch zu suchen. Vielleicht erinnert er sich daran, gewissen Kaufabsichten schon seit langer Zeit zu hegen; er wird vor Ort an diese erinnert und sieht zugleich eine Chance, diese endlich zu realisieren. Deshalb ist es sinnvoll, bei den ungeplanten Käufen nicht nur an „Impulskäufe“ zu denken, was häufig der Fall ist, sondern auch an „Erinnerungskäufe“. Ein Ladenbesucher kann auch vor Ort davon überzeugt werden, dass ein bestimmter Kauf ratsam ist. Deshalb können zu den ungeplanten Käufen auch ,gut überlegte Käufe“ zählen.

Was einem Ladenbesucher durch den Kopf geht, muss mit dem Laden und dem Angebot gar nicht zu tun haben. Er denkt vielleicht über den weiteren Verlauf des Tages nach und über das, was er vor dem Ladenbesuch getan oder erlebt hat. Es kann auch sein, dass sich die Unterhaltung mit Begleitpersonen und mit Mitarbeitern des Geschäfts um ganz andere Dinge dreht. Im Store-TV kommen vielleicht Werbebotschaften regionaler Institutionen (z.B. wirbt die Stadt für ein großes Fest), politische Nachrichten aus aller Welt, Neues vom Sport (z.B. FußballErgebnisse) und der aktuelle Wetterbericht. Dies kann ablenken, kann aber auch Kaufideen induzieren, so z.B. den Kauf einer Eintrittskarte an anderem Ort, bei schlechter Wetterprognose den Kauf eines Regenschirms nebenan oder den Kauf von Speiseeis im selben Geschäft bei der Ansage hochsommerlicher Temperaturen. Damit sei festgehalten, dass auch fremde Themen kaufbezogene Überlegungen auslösen können. Ob diese dann sofort einen Kauf auslösen oder erst bei wiederholten Ladenbesuchen, kann durchaus offen bleiben.

Die Vielfalt möglicher Überlegungen der Besucher beim Besuch eines Geschäfts ist mit den gegebenen Hinweisen nur angedeutet. Sie sind jedenfalls zu 
einem Teil bereits durch Überlegungen vor dem Besuch geprägt, zum anderen Teil aber auch durch das, was sie im Geschäft antreffen oder in Erfahrung bringen können. Vielfach dürfte beides zugleich der Fall sein. Der Ursprung der Gedanken ist jedoch in erster Linie Gegenstand einer Ursachen- oder Determinantenanalyse. Die Erfassung von Gedanken kann sich zwar auch dafür interessieren, wer oder was bestimmte Gedanken ausgelöst hat, muss dies aber nicht.

\section{Mögliche Erfassungsmethoden im Überblick}

Bei einer Erfassung von Gedanken bzw. Kognitionen kommen drei grundlegende Möglichkeiten in Betracht: die „Denke-Laut-Methode“, die „Ex-Post-Befragung“ und die „Gestützte Rekonstruktion“.

Klassischerweise werden Gedanken als Teil des inneren Verhaltens im Nachhinein erfragt (,retrospective verbal reports"). Beim Einsatz der "Befragungsmethode" wird unterstellt, dass die betreffende Person ihr Denken wahrnehmen und erinnern kann, auch dass sie bereit ist, erschöpfende und ehrliche Auskünfte zu erteilen. Erfahrungen zeigen, dass auf diese Weise Gedanken erfasst werden können. Ob jemand sein Denken erinnern kann, hängt jedoch entscheidend davon ab, wie lange der Prozess zurückliegt und wie stark sich das Denken einprägen konnte. Letztes wird vor allem durch das Involvement bzw. die subjektive Bedeutung von Gedanken und durch begleitende Emotionen gefördert.

Soll die Zeit zwischen dem Denken und dem Bericht über das eigenen Denken so knapp wie möglich sein, damit nichts verloren geht, lautet die Methode der Wahl „Denke-Laut-Methode" („,oncurrent verbalization“). Dabei soll die betreffende Person das aussprechen, was sie gerade denkt, was ihr gerade durch den Kopf geht. Erfahrungen zeigen auch hier, dass auf diese Weise Gedanken erfasst werden können. Dabei wird allerdings unterstellt, dass alle Gedanken artikuliert werden können und dass die Artikulation der Gedanken mit der Schnelligkeit der Gedankengänge Schritt halten kann. Zugleich wird hier in Kauf genommen, dass die erbetene Gedankenartikulation das zu erfassende Denken mehr oder weniger stark beeinflusst. 
Soll der Einfluss der Erfassung auf das zu erfassende Denken ausgeschlossen bzw. stark reduziert und der Gefahr begegnet werden, dass sich die betreffende Person nicht mehr erinnern kann, empfiehlt sich eine „Gestütz̧te Rekonstruktion der Gedanken“. Nach dem Vorgang, der erfasst werden soll, werden hier die Gedanken ex post abgefragt, aber die erbetene Erinnerung bzw. die erbetene Rekonstruktion durch geeignete Erinnerungshilfen unterstützt. Eine geeignete Stütze zeichnet sich dadurch aus, dass sie sehr anschaulich an die durchschrittene Situation erinnert, auch an die Dynamik des Geschehens, und dass sie so gestaltet wird, dass die Gefahr so gering wie möglich ist, dass die Auskunftsperson statt Erinnerungen etwas „fabriziert“, das mit den zu rekonstruierenden Gedanken wenig gemein hat.

Aus dem Gesagten geht schon hervor, welche Kriterien bei der Beurteilung der skizzierten Methoden in Frage kommen: zum einen die interne Validität, zum anderen die externe Validität. Bei der internen Validität stellt sich die Frage, ob das was gemessen werden soll, die Gedanken nämlich, auch tatsächlich gemessen wird. Das kann auch dann der Fall sein, wenn die Gedanken per Erfassung beeinflusst worden sind. $\mathrm{Ob}$ das Gemessene auf vergleichbare Situationen oder Personen übertragen werden kann, in denen keine Messung erfolgt, ist eine Frage der Generalisierbarkeit bzw. der externen Validität. Diese ist beeinträchtigt, wenn die Messung an sich das zu untersuchende, innere Verhalten beeinflusst und wenn sich nur bestimmte, nicht-repräsentative Personen für eine Erfassung ihres Verhaltens bereit erklären. Je größer die Reaktivität der Personen und je größer die Selektivität der Stichprobe ausfallen, desto geringer ist die Generalisierbarkeit bzw. die externe Validität der Befunde.

\section{Die relevanten Befragungsmethoden}

Bei der Befragung von Ladenbesuchern, die in Erfahrung bringen möchte, was ihnen bei ihrem Besuch durch den Kopf gegangen ist, gibt es mehrere Möglichkeiten.

- Erstens die Ex-post-Befragung im unmittelbaren Anschluss an den Ladenbesuch, die sich auf die Gedanken während des gesamten Besuchs bezieht. 
- Zweitens die Ex-post-Befragung, die sich nur für bestimmte Teile der Gedanken beim Ladenbesuch interessiert, so z.B. für die Gedanken in bestimmten Arealen, an bestimmten Regalen oder bei der Betrachtung von werblichen Hinweisen an dem Wänden, an Theken oder per Store TV, für die Gedanken bei bestimmten Produktkäufen oder für Gedanken, die für die Person eine besondere Bedeutung haben.

- Drittens die Unterbrechermethode, bei der ein Besuch unterbrochen wird, um die betreffende Person zu fragen, was ihr gerade eben - z.B. beim Betrachten einer Auslage oder kurz vor der Mitnahme eines Produktes - durch den Kopf gegangen ist (McIntyre \& Bender 1986).

Diese Befragungsvarianten unterscheiden sich in der Anforderung an die Gedächtnisleistung und damit in der Gefahr, den Befragten zu überfordern und „Erinnerungen“ zu fabrizieren (vgl. dazu den Begriff der „interpretive reports“ bei Fidler 1983 S. 429). Es leuchtet unmittelbar ein, dass die POS-Forschung bislang keine Versuche unternommen hat, das gesamte kognitive Geschehen während des Ladenbesuches per Nachherbefragung von Ladenbesuchern in Erfahrung zu bringen. Weniger verständlich ist dagegen der Mangel an Studien, die sich nur für ausgewählte Aspekte des kognitiven Prozesses am POS interessieren und dabei z.B. die Unterbrechermethode eingesetzt haben.

Es könnte nun die Selbstaufmerksamkeit beim Ladenbesuch gesteigert werden, indem die Besucher beim Eintritt in das Geschäft gebeten werden, sich ihre Gedanken bewusst zu machen und beim Verlassen des Geschäfts zu Protokoll zu geben. Auf diese Weise lassen sich die Besucher in ihrer Selbstwahrnehmung sicherlich sensibilisieren und damit Erinnerungsschwächen reduzieren. Dies müsste allerdings mit einer Einschränkung der externen Validität erkauft werden, weil eine Vorabsensibilisierung das Denken und Handeln beeinflusst (vgl. Russo, Johnson \& Stephens 1989 S. 765). Vielleicht sinkt durch die Vorab-Ansprache auch die Bereitschaft, an der Nachbefragung teilzunehmen, so dass die Selektivität der Stichprobe ansteigen würde. 


\section{Varianten der Denke-Laut-Methode}

Die Denke-Laut-Methode (DLM) wird häufig als der klassische und unmittelbare Zugang zu kognitiven Prozessen gesehen (Ericson \& Simon 1980, Weidle \& Wagner 1982, Wallendorf \& Brucks 1993). Dies trifft nicht nur für die allgemeine Entscheidungsforschung zu, sondern auch für die Käuferforschung am POS. Zu den Autoren, die mit der DLM bei Ladenbesuchern gearbeitet haben, zählen Payne \& Ragsdale (1978), Titus \& Everett (1996) und Reicks et al. (2003).

Auch wenn immer nur von „der Denke-Laut-Methode“ gesprochen wird, so sind doch mehrere Varianten zu unterscheiden und unterschiedlich zu beurteilen. In Bezug auf die zu untersuchenden Gedanken wäre am POS z.B. zu unterscheiden, ob eine Person alle ihre Gedanken artikulieren soll oder nur solche, die mit geplanten, getätigten oder revidierten Käufen in Beziehung stehen. Varianten der DLM unterscheiden sich auch in der Protokollierung der "lauten“ bzw. ausgesprochenen Gedanken. Üblicherweise werden Tonaufzeichnungsgeräte in der Hand des Probanden oder in der Hand einer Begleitperson eingesetzt, selten handschriftliche Notizen des Forschers. Wenn trotz solcher Aufzeichnungsgeräte Begleitpersonen eingesetzt werden, besteht deren Aufgabe darin, das Erlahmen des lauten Denkens zu erkennen und das ununterbrochene laute Denken sicher zu stellen. Da eine Begleitperson das Verhalten des Probanden beeinflussen und ein lautes Aussprechen von Gedanken bei Personen ohne Begleitung Drittpersonen verwundern und zu Gaffereffekten führen kann, liegt es nahe, Handys einzusetzen und die Gedanken in ein Handy sprechen zu lassen (Büttner 2008 S. 126f.). Auf diese Weise kann der Proband mit dem Forscher in Verbindung stehen und außerdem die Möglichkeit genutzt werden, den Probanden am anderen Ende der Leitung bei Bedarf zur fortgesetzten Artikulation seines Denkens zu ermuntern.

Zweifel an der Eignung der DLM stellen darauf ab, dass Probanden mit der Artikulation ihrer Gedanken überfordert sein können, dass die Artikulation der Gedanken mit dem Denken nicht Schritt halten kann. Nicht alles, was einer Person durch den Kopf geht, seien es Bilder, Symbole, Namen, Ideen, Erinnerungen und 
Selbstwahrnehmungen, auch das Gefühl, die Erwartungen anderer in Betracht zu ziehen, läßt sich immer, jederzeit und von jedermann in verständliche Worte fassen (vgl. Genest \& Turk 1981 p. 243). Es ist auch zu fragen, ob immer unterstellt werden darf, dass die Bereitschaft, seine Gedanken preiszugeben, gegeben ist. Diese Bereitschaft dürfte vor allem dann auf Grenzen stoßen, wenn der Aufwand von der Auskunftsperson (AP) als zu hoch empfunden wird, wenn Tabuthemen involviert sind und wenn das Gedankenerfassen als ein Eingriff in die Privatsphäre angesehen wird.

Eine Überforderung von Probanden und Motivationsdefizite können zu lückenhaften und/oder unrichtigen Angaben führen. Unrichtige Angaben können gewollt sein, so z.B. als gezielte Irreführung, als Selbstschutz bei Tabuthemen oder als Wunsch, doch etwas zu sagen zu haben. Unrichtige Angaben können aber auch ohne den Willen dazu zustande kommen. Dies ist z.B. der Fall, wenn Wahrnehmungen durch Vermutungen bzw. Annahmen ersetzt werden. Auf solche Fabrikationsprozesse bzw. Artefakte haben u.a. Nisbett \& Wilson (1977 S. 233) hingewiesen. Sie verweisen darauf, dass der Fabrikation von Gedanken, die gar nicht gedacht wurden, sog. naive Theorien darüber zugrunde liegen, was ein Mensch in vergleichbaren Situationen normalerweise denkt bzw. nicht denkt (S. 248).

Bedenken bestehen auch bezüglich der externen V alidität jener Befunde, die mit der DLM zu Tage gefördert werden. Diese Bedenken beruhen vor allem auf der Annahme, dass von der DLM eine erhebliche Beeinflussung oder Veränderung der Verhaltenssituation und somit auch des Denkens einhergeht. Lautes Denken ist nicht alltäglich, nicht gewohnt. Das Just-in-Time-Aussprechen von Gedanken bindet Ressourcen, behindert Wahrnehmungen, kann aber auch Lernchancen eröffnen. Die DLM kann aber auch dazu motivieren, ein gutes Bild abzugeben, eine gute Leistung zu erbringen (Russo, Johnson \& Stephens 1989 S. 764f.). Sehr wahrscheinlich ändert sich das Involvement der Probanden allein schon dadurch, dass sie ihr Denken bewusster wahrnehmen und einer dritten Person mitteilen 
sollen. Studien zeigen zumindest eines ganz deutlich: die Probanden benötigen bei der DLM mehr Zeit für die Aufgabenbearbeitung (vgl. Reicks et al. 2003 S. 160).

Die externe Validität ist aber auch durch Selektivitätseffekte gefährdet: Nicht alle Zielpersonen sind bereit, an solchen Erhebungen teilzunehmen. Es mag ihnen nicht nur an der erforderlichen Zeit fehlen; Bedenken dahingehend, dass es sich um einen Eingriff in die Privatsphäre handelt oder dass die erforderliche Selbstoffenbarung mit eine Blamage verbunden sein könnte, kommen hinzu. Versuche, die derartige Hemmnisse reduzieren sollen, bestehen i.d.R. darin, dass Anreize zum Mitmachen eingesetzt werden, nicht nur finanzielle Anreize, sondern auch immaterielle.

Um die Möglichkeiten und Grenzen einer begleitenden Erfassung von Gedanken beim Ladenbesuch näher zu betrachten, wollen wir drei Versuche dieser Art näher betrachten: die Studie von Titus \& Everett (1996), die Untersuchung von Payne \& Ragsdale (1978) und die Validierungsstudie von Büttner (2008).

Titus \& Everett (1996 S. 265f.) interessierten sich für die Produktsuche im Laden sowie für jene Prozesse, die diesem Verhalten zu Grunde liegen. Sie sehen das Einkaufen vornehmlich als eine Suche der gewünschten Produkte, als ,in-store navigation“ und als „wayfinding“ (Downs \& Stea 1973, Passini 1984): “the process of completing these search tasks, referred to as wayfinding, comprises a significant portion of the consumers retail shopping experience." (Titus \& Everett 1996 S. 265). Deshalb konzentrierte sich die Studie von Titus \& Everett (1996) auf die Produktsuche im Laden und auf die zu Grunde liegenden Orientierungen (S. 266). Die teilnehmenden Probanden erhielten eine Einkaufsliste mit 21 Produkten, die sie im ausgewählten Supermarkt auffinden sollen und nennen dies eine „Kaufsimulation“ (S. 267). Die Teilnehmer bekamen ein Mikrophon umgehängt und sollten aber nicht nur ihre Gedanken, sondern auch ihr Lauf- bzw. Suchverhalten beschreiben (S. 267). Die Probanden sollten sagen, (1) wo sie suchen, (2) warum sie dort suchen und (3) welche Anhaltspunkte (features) in der Ladenumgebung sie dabei nutzen. Den Probanden wurde ein Begleiter mitgeschickt, der offensichtlich 
falsche Angaben zu monieren hatte (S. 267): „A trained investigator accompanied each subject, recording their search route and employing standardized verbal prompts when obvious lapses in subject verbalization occured.“

Für die keineswegs leichte Aufgabe, die einzelnen Gedanken sowie das eigene Lauf- und Suchverhalten just-in-time zu formulieren und auf Mikrophon zu sprechen, waren bereitwillige Probanden zu finden. Angeboten wurden ein 10Doller-Gutschein (US-\$ 10 gift certificate) und 5 Dollar in bar (S. 269). Auf diese Weise konnten 66 Personen zur Teilnahme bewegt werden; drei davon wurden wegen einer Fehlfunktion des Recorders aus der Auswertung ausgeschlossen (S. 269).

Auch die Auswertung der Protokolle orientierte sich am Konzept des „Suchverhaltens“. Das in mehreren Stufen entwickelte Coding Scheme enthielt sieben Suchaufgaben, sechs Suchfehler und zwei Suchmodi. Den Suchmodi "passive search mode" (search sequence proceeds from the environment to the shopping list) und "active search mode" (search sequence proceeds from the shopping list to the environment) waren die aufgezeichneten Statements der Probanden zuzuordnen (S. 271). Eine statistische Auswertung der Befunde erfolgt nicht. Stattdessen liefern die Autoren detaillierte Angaben zur Kategorisierung der einzelnen Statements und Beobachtungen (S. 271-283), was bei künftigen Analysen des Suchverhaltens im Laden sicherlich hilfreich sein dürfte.

Eine weitere Gedankenprotokollierung bei Ladenbesuchern stammt von Payne \& Ragsdale (1978). Die Autoren interessierten sich für die kognitiven Prozesse beim alltäglichen Einkaufsverhalten, vor allem für die Entscheidungsprozesse „verbal protocols, to investigate consumer decision making in supermarkets“. Die Hausfrauen und Mütter schulpflichtiger Kinder, regelmäßige Käufer von Süßigkeiten („heavy purchasers of candy“), hatten eine zweifache Bitte zu erfüllen: Zum einen sollten sie mit insgesamt sechs wöchentlichen Haupteinkaufstrips (six major weekly shopping trips) an der Studie teilnehmen. Zum anderen sollten sie in Begleitung einer Studentin (accompanied by female graduate students) während des 
Ladenbesuchs bzw. der Süßigkeitenabteilung „laut denken“. Der Artikulierungsauftrag ging auch in dieser Studie über das Verbalisieren der Gedanken weit hinaus. Dies zeigt der Text, den die Begleitperson zu Beginn vorzulesen hatte: „We want you to think aloud while shopping. Say what you are doing and where you are going in the store. Whenever you see something as you are walking through the store, mention it. Talk about the products and brands that you are trying as well as those you notice but do not buy. For instance, say what observations you are making about brands, their locations in the store, package size, price, ingredients, flavours, and so on. Remember, say anything that comes to your mind no matter how unimportant it seems to you" (S. 572). Die Frauen hatten somit nicht nur ihre Gedanken mit Hinweisen auf die relevanten Produkte (Marken, Artikel) sowie auf deren Merkmale und deren Standort laut auszusprechen, sondern auch das eigene Verhalten bzw. den eigenen Laufweg sowie alles, was dabei ins Blickfeld gerät, also auch die ganze Wahrnebmung. Dies macht es verständlich, dass die Begleitpersonen auch die Aufgabe erhielten, die Probanden an ihre DenkeLaut-Aufgabe immer wieder zu erinnern. Für die Teilnahme an dieser Studie wurde ein Honorar von 30 US-Dollar versprochen und bezahlt (S. 572).

Die Schwierigkeit der Aufgabe und das damit verbundene Risiko einer Überlastung der Probanden muss auch den Autoren bewusst gewesen sein, denn als Vorbereitung wurden die AP insgesamt drei Trainingsaktionen unterzogen: Das erste Artikulationstraining erfolgte bei den Frauen zu Hause am Beispiel der Lektüre einer Werbebroschüre. Als zweites Training diente der erste Einkaufstrip und als drittes Training der zweite Geschäftsbesuch (S. 572). Für die Studie i.e.S. wurden dann nur die letzten vier Einkaufstrips ausgewertet.

Nach einer Übersetzung der Protokolle in einzelne Elemente (into single statements or phrases) konnten diese einer Auswertung unterzogen werden. Dabei zeigte sich, dass die Anzahl der aus den Protokollen heraus gelesenen Statements pro Person zwischen 1300 und 3000, im Durchschnitt bei knapp 2000 lag (S. 574). $\mathrm{Ob}$ die Auswertung der Statements aus diesem Grunde oder aus anderen Gründen 
auf 10 Personen beschränkt wurde, lassen die Autoren offen. Denkbar wäre, dass sich von den insgesamt 19 akquirierten Hausfrauen nicht alle zu den sechs erwünschten Einkaufstrips gemeldet hatten.

In der Validierungsstudie von Büttner (2008) hatten ausgewählte, vorab angeworbene Ladenbesucher bei ihrem Besuch eines Elektrofachmarktes ihre Gedanken in ein Handy zu sprechen und so den Eindruck zu erwecken, als sprächen sie mit einem Bekannten. Die Kontrollgruppe hatte keine Kopfkamera zu tragen und auch keine Gedanken zu artikulieren. Ein Teil der Probanden sollte in der CD-Abteilung etwa 15 Minuten überbrücken bis zum imaginären Treffen mit einem Freund, der andere Teil sollte in etwa 15 Minuten in der CD-Abteilung für einen Freund bzw. eine Freundin eine CD als Geschenk aussuchen (S. 130f.). Die Befunde zeigen, dass mit der Gedankenartikulation - wie erwartet - eine größere Selbstaufmerksamkeit einhergeht - bei Seekern ebenso wie bei Surfern (S. 145). Demgegenüber fällt in beiden Gruppen der Einkaufspass geringer aus, nicht nur die Anzahl der positiven Emotionen, sondern auch die Stimmung (S. 145). Auch die Neigung zu ungeplanten Käufen ist bei der Gedankenartikulation geringer ausgeprägt als in der Kontrollgruppe (ebd). Eine Konstruktvalidierung der DLM sieht der Autor darin, dass bezüglich der Kognitionen erwartete Unterschiede auch tatsächlich festgestellt werden konnten: Im Vergleich zu den Surfern berichteten die Seeker mehr Gedanken zur Orientierung, zu Zielen bzw. Intentionen, zur Zielverfolgung und zur Zielerreichung, weniger dagegen zur Produktwahrnehmung, zur Produktbewertung und zur Produktauswahl (S. 173f.).

Die soeben angesprochenen Studien machen drei Sachverhalte deutlich: Erstens die Aussagekraft bzw. den heuristischen Wert der DLM, zweitens deren Aufwendigkeit und drittens die Grenzen für die interne und externe Validität dieser Methode. Allerdings sei auch festgehalten, dass sich die Belastung der Probanden bei künftigen Studien dadurch begrenzen lässt, dass immer nur um die Artikulierung der aktuellen Gedanken, vielleicht auch nur der Gedanken in einem bestimmten Areal, gebeten wird, und nicht auch noch um die Artikulierung des 
Verhaltens und sämtlicher Wahrnehmungen. Die Aufgabe, alles auszusprechen, was gerade durch den Kopf geht, ist schwierig, belastend und beeinflussend genug.

\section{Die gestützte Rekonstruktion kognitiver Prozesse}

Soll ein Ladenbesucher während des Ladenbesuchs möglichst unbeeinflusst agieren können und dennoch die Chance bestehen, dass seine Gedanken erfasst werden können, kommt die gestützte Rekonstruktion in Frage. Dabei sind geeignete Gedankenstützen zu wählen, anzufertigen und einzusetzen. Sie können nach dem Besuch eines Geschäfts oder einer Abteilung zum Einsatz kommen, wenn gefragt wird: „Und was ist Ihnen dabei durch den Kopf gegangen?“

\subsection{Varianten einer wirksamen Gedächtnisstütze}

Die Methode der gestützten Abfrage bzw. des „aided recall“ von zurückliegenden, nicht leicht erinnerbaren Sachverhalten ist aus anderen Bereichen der Markt- und Sozialforschung durchaus bekannt. Dennoch stellt sich zunächst die Frage, welche Gedankenstützen bei der Rekonstruktion kognitiver Prozesse beim Ladenbesuch in Frage kommen. Bei der Analyse komplexerer Phänomene wie z.B. extensiver Problemlösungsprozesse kennt die Forschung unterschiedliche Gedankenstützen, so z.B. die wiederholte Vorlage der Problemstellung, die Rekonstruktion des vom Probanden gewählten Problemlösungsweges, die Vorlage der vom Probanden erarbeiteten Problemlösung und die Wiedergabe jener Bedingungen, in denen die Problemlösung stattgefunden hat. Einige dieser Stützen tragen dem Umstand Rechnung, dass ein dynamisches Geschehen - nämlich Gedankenabfolgen rekonstruiert werden soll und deshalb auch die Gedankenstütze der Dynamik des Geschehens Rechnung tragen sollte.

Bei der Erfassung kognitiver Prozesse beim Ladenbesuch bieten sich verschiedene Möglichkeiten an. Es können z.B. Fotos oder Skizzen jener Areale und jener Präsentationsflächen gezeigt werden, die der Proband aufgesucht hat. Denkbar wären auch Skizzen des Kundenlaufs, Einträge des Laufverhaltens in einen Ladengrundriss. Beide Dinge lassen sich durchaus gut kombinieren. Bei einer 
verdeckten oder offenen Beobachtung des Ladenbesuchers wäre auch an die verbale Kommunikation des Beobachtungsergebnisses als Gedankenstütze zu denken. Dasselbe gilt dann auch für Videoaufnahmen. Diese könnten Aufnahmen des Lauf- und Zuwendungsverhaltens enthalten, die ein mitlaufender Kameramann aufgenommen hat, aber auch Aufnahmen des Blickfeldes beim Einsatz einer kleinen Kopfkamera. Sind auf diesem Wege Verhaltensweisen wie Kundenlauf und visuelle Zuwendungen erfasst worden, können die Aufnahmen auch als Gedankenstützen und damit in einer sog. Zweitverwertung zum Einsatz kommen.

Der Vergleich mit der DLM macht deutlich, dass es bei der gestützten Rekonstruktion nicht um eine sofortige, sondern um eine nachträgliche Artikulation von Gedanken handelt. Ist bei der DLM von einer Beeinflussung des Verhaltens am POS die Rede gewesen, so muss dies bei der Erstellung einer angemessenen Gedankenstütze nicht der Fall sein. Eine Beeinflussung findet dann nicht statt, wenn die Erkenntnisse aus einer verdeckten Beobachtung stammen. Anders liegt der Sachverhalt, wenn Notizen eines Begleiters im Rahmen der offenen Beobachtungen und Filmaufnahmen des Begleiters oder Aufnahmen einer Blickfeldkamera als Gedankenstütze erstellt werden. Hier müssen die Probanden zustimmen, so dass auch mit Selektivitätseffekten zu rechnen ist.

\subsection{Die videogestützte Gedankenrekonstruktion}

Die videogestützte Gedankenrekonstruktion beim Ladenbesucher hat den Vorteil, anschauliche und dynamische Gedankenstützen einsetzen zu können (Silberer 2005, Silberer \& Büttner 2008, Büttner \& Silberer 2008). Sie werden auch dem Lauf- und Zuwendungsverhalten des jeweiligen Probanden gerecht, denn sie sind „personalisiert“. Sie enthalten aber nicht nur Hinweise auf das Verhalten des Probanden, sondern auf Teile der jeweiligen Verhaltenssituation. Und sie haben auch den Vorteil, dass sie auch die akustischen Elemente einer Verhaltenssituation wiedergeben - nicht nur Geräusche, sondern auch Durchsagen, Hintergrundmusik, Aussagen von Auskunftspersonen und Kommentare der Kaufbegleitung zum Beispiel. 
Kommt bei der Gedankenabfrage bzw. bei der Gedankenrekonstruktion ein Video als Gedächtnisstütze zum Einsatz, lässt sich bei der Auswertung der Angaben des Probanden zu einem späteren Zeitpunkt auch der Umstand nutzen, dass die Angaben im Lichte des Videos - also als eine Kombination - analysiert und interpretiert werden können. Erfolgt die Gedankenrekonstruktion im virtuellen Tonstudio, das sich heutzutage auf einem Laptop durchaus realisieren lässt, können die Angaben auf einer zweiten Tonspur des Videos so abgespeichert werden, dass auch die beiden Zeitschienen - die des Videos und die der Gedankenabfrage zueinander in Bezug gesetzt werden können.

Um die Durchführbarkeit und Aussagekraft der videogestützten Gedankenrekonstruktion am POS zu überprüfen, führten wir eine Studie in einem innerstädtischen Elektrofachmarkt durch, der sich über zwei Etagen erstreckt. Das erste Ziel dieser Pilotstudie bestanden darin, die technische Machbarkeit und die Akzeptanz einer videogestützten Gedankenrekonstruktion (VGR) bei allen Beteiligten - beim Management, bei den Besuchern und bei den Mitarbeitern - zu untersuchen. Außerdem war beabsichtigt, erste Anhaltspunkte in Bezug auf die interne und externe Validität der videogestützten Gedankenrekonstruktion zu gewinnen.

Mit Hilfe der modernen Video- und PC-Technik gelang es, die Filmaufnahmen eines unauffällig mitlaufenden Kameramannes, denen vor allem jüngere Besucher zustimmten, unmittelbar nach dem Verlassen des Geschäfts bzw. am Ausgang auf ein Laptop zu übertragen und sofort - noch während des Download von der Kamera auf das Laptop - mit der Präsentation zu beginnen. Außerdem gelang es, die von den Probanden artikulierte Gedankenrekonstruktion so aufzunehmen, dass nicht nur Stopps des ablaufenden Videos möglich waren, sondern auch eine spätere Zuordnung der Zeit- und Tonspuren gesichert war.

Erfreulich war auch die Akzeptanz bei allen Beteiligten. Das Management hätte unser Vorhaben mit dem Argument ablehnen können, es wolle keine Belästigung der Kunden und/oder keine Verunsicherung durch ggf. erkennbare Film- 
aufnahmen, stimmte aber unserem Vorhaben zu. Das Interesse an den Ergebnissen war ebenfalls vorhanden. Auch die Mitarbeiter fühlten sich nicht in ihren persönlichen Interessen verletzt. Der Betriebsrat hätte das Vorhaben wegen der Gefahr, dass solche Interessen dort verletzt werden, wo Mitarbeiter in den Videos auftauchen, durchaus ablehnen können. Schließlich stießen wir auch auf die Bereitschaft recht vieler Ladenbesucher, an dieser Studie teilzunehmen. 128 Besucher, vorwiegend jüngere, waren zur Mitarbeit bereit, ohne dass ein Honorar versprochen bzw. ausgezahlt werden musste.

In Sachen externe V alidität können zwei Dinge festgehalten werden: Ein Viertel der angesprochenen Personen waren bereit, an der Studie teilzunehmen, vor allem jüngere Ladenbesucher. Da eine Nachbefragung angekündigt war, führten auch Zeitgründe zur Absage, nicht nur Bedenken zum Verfahren an sich. Angaben zum empfundenen Störpotential der Videoaufnahme lassen erkennen, dass sich die Wahrnehmung einer außergewöhnlichen Situation und die so bedingte Reaktivität im Verhalten der beobachteten Ladenbesucher in engen Grenzen halten. 33 \% der gefilmten Besucher gaben an, „gar nicht“ gestört worden zu sein. Nur eine einzige Person gab an, sich „sehr stark“ gestört gefühlt zu haben. Ein großer Teil - ca. 40 \% - bekundete, dass er sich „kaum“ gestört fühlte. 20 der insgesamt 128 Personen fühlten sich „mehr oder weniger stark“ gestört.

Hinweise bezüglich der internen V alidität betreffen sowohl die Datenauswertung, nämlich die Übereinstimmung der Ergebnisse bei zwei gleichzeitig eingesetzten Codierpersonen (80 \% der Codes stimmten überein), als auch die Ergebnisse einer Known-Group-Validierung. Da bekannt war, wer mit welcher Absicht den Laden betreten hatte, konnten die rekonstruierten Gedanken bei der Seeker-Gruppe mit denen der Surfer-Gruppe verglichen werden.

Anhand einschlägiger Konzepte und Studien wurde Folgendes erwartet: In komplexen Ladenumwelten (wie im Untersuchungsbeispiel gegeben) müssen sich die Seeker stärker orientieren als die Surfer, um zu ihrem Ziel zu kommen. Sie werden sich mehr als Surfer Gedanken zur Zielverfolgung machen und mehr 
Probleme bei der Zielverfolgung wahrnehmen. Sie gehen selektiver vor und müssen sich daher in Sachen Alternativensuche, -wahrnehmung, -beurteilung und vergleich nicht so viele Gedanken machen, wohl aber mehr Gedanken zur Alternativenauswahl, denn sie sind ja gekommen, um eine gute Alternative bzw. eine zielführende Lösung zu wählen. Auch sollten die Kaufwilligen mehr darüber nachdenken, ob und inwieweit sie ihr ursprünglich gefasstes Ziel erreicht, ihre anfängliche Absicht realisiert haben.

Wie die per Inhaltsanalysen erarbeiteten Befunde zeigen, werden diese Erwartungen bestätigt. Die festgestellten Unterschiede zeigen, dass mit der VGR bei Ladenbesuchern aussagekräftige Ergebnisse erzielt werden können. Dafür müssen - je nach dem, wie die Gedankenstützen erstellt und eingesetzt werden Beschränkungen der externen Validität in Kauf genommen werden.

Eine neuere Validierungsstudie befasste sich mit der Gedankenrekonstruktion anhand von Blickfeldaufnahmen. In der oben bereits angesprochenen Studie von Büttner (2008) wurde nicht nur eine DLM-Gruppe, sondern auch eine VGRGruppe untersucht, die ebenfalls in Surfer und Seeker unterteilt war. Deshalb erlaubt diese Studie einen Vergleich der DLM mit der VGR-Methode. Die Resultate zeigen unter anderem Folgendes: Die VGR-Methode belastet die Probanden bei ihrer angestammten Aufgabe beim Ladenbesuch weniger stark als die DLM, denn es zeigte sich, dass das Personal von den VGR-Gruppe weniger häufig angesprochen und um Hilfe gebeten wurde (S.154f.). Die VGR-Gruppe berichtete außerdem weniger negative Emotionen, allerdings betrifft dies allein die Surfer. Dasselbe betrifft die Neigung zu ungeplante Käufen (S. 157f.). Allerdings wurden in der VGR-Gruppe pro Zeiteinheit der Besuchsdauer weniger Gedanken berichtet als von DLM-Gruppe (S. 166f.). Dafür enthielten die Berichte der VGRGruppe mehr Äußerungen, die sich auf die Erklärung und Rechtfertigung von Überlegungen beziehen (S. 168). Dies erklärt sich nicht nur aus der Tendenz, die eigenen Überlegungen zu erklären, sondern auch daraus, dass - anders als bei der DLM - die Zeit dafür zur Verfügung stand. Deutlich ausgeprägte Unterschiede 
betreffen die Anteile der unmittelbaren, deskriptiven und reflexiven Gedankenverbalisierung. Der Anteil unmittelbarer Gedankenäußerungen war in der VGR-Gruppe geringer, der Anteil deskriptiver und reflexiver Verbalisierungen dagegen höher (S. 170). Orientierungsbezogene Gedanken und Gedanken zur Produktsuche waren bei den Seekern in der VGR-Gruppe häufiger als bei den Seekern in de DLM-Gruppe, Gedanken zu Zielen bzw. Intentionen, Gedanken zur Zielerreichung und Gedanken zur Produktwahrnehmung dagegen seltener. Und bei den Surfern in der VGR-Gruppe waren die Gedanken zur Produktwahrnehmung seltener als in der DLM-Gruppe, Gedanken zur Produktsuche dagegen häufiger (S. 174).

Diese Befunde belegen - wie erwartet - die bei einer VGR geringer ausprägte Reaktivität, aber auch die deutlich häufigeren Versuche, das eigene Denken zu erläutern und zu begründen. Unmittelbare Artikulationen bezüglich der Gedanken sind bei der DLM offenbar eher zu erwarten. Die Begründungsversuche sollten aber nicht als Hinweise auf eine geringe Validität der rekonstruierten Gedanken gewertet werden, sondern lediglich als Aufforderung an die Auswertung von Gedankenprotokollen, zwischen Hinweisen auf Gedanken und deren Begründung zu unterscheiden. Die Ergebnisse bei Büttner (2008) zeigen aber auch, dass Seeker sowohl bei der VGR-Methode als auch bei DLM andere Gedanken berichten als Surfer, was eher für als gegen die beiden Verfahren spricht.

\section{Möglichkeiten einer Methodenkombination}

Die Kombination von Messmethoden bei der Erfassung kognitiver Prozesse beim Ladenbesuch kann aus verschiedenen Gründen heraus erfolgen: zum einen, um deren interne und externe Validität zu überprüfen, zum anderen, um eine Art Arbeitsteilung zu erreichen, wenn die einzelnen Methoden Unterschiedliches messen. Letzteres soll uns hier kurz beschäftigen. Dabei wollen wir zwischen einem gruppeninternen Methoden-Mix und einem gruppenübergreifenden Methoden-Mix unterscheiden. 


\subsection{Zum gruppeninternen Methoden-Mix}

\section{(1) Zur Kombination diverser Befragungsmethoden}

Soll der gesamte kognitive Prozess während eines Ladenbesuchs und speziell der in einem bestimmten Areal ablaufende Gedankengang interessieren, bietet es sich an, am betreffenden Areal die Unterbrechermethode einzusetzen und beim Verlassen des Geschäfts das Erfragen besonders wichtiger Gedanken, bezogen auf den gesamten Ladenbesuch. Das Selektive in der Abschlussbefragung könnte dazu beitragen, dass die Erinnerungsfähigkeit des Probanden nicht überfordert und dessen Zeit nicht allzu sehr beansprucht wird. Denkbar wäre auch ein Vorgehen, bei dem die Unterbrechermethode bei einem Teil der Probanden und die ExPostbefragung am Ausgang bei einem anderen Teil zum Einsatz kommen.

\section{(2) Zur Kombination diverser Denke-Laut-Methoden}

Sind beim Einsatz der DLM nur ein Teil der Ladenbesucher bereit, ihr Denken wie in einem Telefongespräch zuartikulieren, bietet es sich an, die Handymethode ebenso einzusetzen wie die klassische DLM per Kaufbegleitung und Tonbandaufzeichnung oder Diktaphon - allerdings nach Teilpopulationen getrennt. Ein solches Vorgehen empfiehlt sich auch, wenn es darum geht, den Einfluss einer „physischen Kaufbegleitung“ auf das Verhalten des Ladenbesuchers abzuschätzen bzw. mit dem Einfluss der „fernmündlichen Kaufbegleitung“ vergleichen zu können.

\section{(3) Zur Kombination diverser Varianten der Gedankenrekonstruktion}

Die Varianten der gestützten Gedankenrekonstruktion unterscheiden sich vor allem in der gewählten Erinnerungsstütze. Diesbezüglich war oben von Laufskizzen, von Fotos zur Warenpräsentation und von Aussagen eines Beobachters die Rede. Eine Methodenkombination könnte nun darin bestehen, dass bei der Rekonstruktion von Gedanken bei der Orientierung im Geschäft und bei der Wahl einzelner Areale Laufskizzen als Gedankenstütze zum Einsatz 
kommen und bei der Rekonstruktion von Gedanken bei der Betrachtung bestimmter Regale die entsprechenden Regalfotos. Sind einem Beobachter unerwartete, ungewöhnliche Verhaltensweisen aufgefallen, ließe sich der Einsatz der soeben genannten Methoden auch durch ein Gespräch ergänzen, in dem der Beobachter diese Verhaltensweisen zur Sprache bringt und auch hier die klassische Frage stellt: „Was ist Ihnen dabei durch den Kopf gegangen?“

\subsection{Zum gruppenübergreifenden Methoden-Mix}

Auch hier sollen nur ausgewählte Beispiele für eine Methodenkombination angesprochen werden. Ein gruppenübergreifendes Methoden-Mix könnte z.B. darin bestehen, dass für die Phase des Eintretens in ein Kauf- oder Warenhaus die telefonische DLM zum Einsatz kommt, zumal in dieser Phase wenig zu tragen und zu schieben ist, für die Gedanken vor einem Regal, an dem ein Produkt definitiv gewählt und mitgenommen wurde, die anhand des Regalfotos gestützte Gedankenrekonstruktion gewählt wird und in Bezug auf die sonstigen Gedanken die selektive Nachherbefragung zum Einsatz kommt. In diesem Falle erfolgte die Gedankenerfassung in Bezug auf die Eintrittsphase just-in-time, die Ex-postAbfrage selektiver Gedanken sowie die fotogestützte Gedankenrekonstruktion jedoch erst beim Verlassen des Geschäfts.

Interessieren allein die Gedanken in einer bestimmten Abteilung, so könnte folgendes Vorgehen gewählt werden: Erfassung aller Gedanken in der besuchten Abteilung anhand der Unterbrechermethode, so dass der Besucher beim Verlassen der Abteilung gebeten werden muss, all das zu berichten, was ihm in der soeben besuchten Abteilung durch den Kopf gegangen ist, und die gestützte Gedankenrekonstruktion in Bezug auf jene Produktpräsentationen, die der Besucher kontaktiert hat, wobei z.B. vorab erstellte Fotos von allen relevanten Regalen, Truhen, Wühltischen und/oder Fremdbedienungstheken zum Einsatz kommen können. 


\section{Zusammenfassung und Ausblick}

Die Bedeutung kognitiver Prozesse beim Ladenbesuch für den Kauferfolg des Kunden und den Absatzerfolg des Anbieters ist unbestritten. Dennoch kann von einer intensiven Erforschung dieser Prozesse keine Rede sein - weder in der Wissenschaft, noch in der Praxis. Die Schwierigkeiten und der Aufwand, der mit entsprechenden Analysen verbunden sind, mag eine Erklärung für den derzeitigen Wissensstand abgeben. Im harten Wettbewerb um Kunden wird die Bedeutung einschlägiger Kenntnis ebenso zunehmen wie das Interesse an Befunden einer einschlägigen Verhaltensforschung am POS. Die Aussagekraft der künftigen Forschung kann gesteigert werden, wenn das breite Methodenarsenal beachtet, reflektiert, verbessert und verstärkt eingesetzt wird. So vielfältig die Gedankengänge im Kopf des Ladenbesuchers sein können, so vielfältig sind auch die Methoden, die bei einer Erfassung von Gedanken zum Einsatz kommen können. Diese Vielfalt aufzuzeigen und auch Kriterien anzusprechen, die bei Beurteilung einzelner Methoden herangezogen werden sollten, war die zentrale Aufgabe dieses Beitrags.

Es kommt nun darauf an, die Vielfalt möglicher Gedanken beim Besuch eines Geschäfts verstärkt zu reflektieren, das relevante Methodenarsenal zu erweitern und zu verbessern und die Erfassung kognitiver Prozesse beim Besuch eines Ladengeschäfts zu intensivieren. Der Wissenschaft sollte es vornehmlich darum gehen, eine geeignete Messtheorie zu entwickeln, die es uns erlaubt, im Bereich der Gedankenerfassung am POS fundierte Validitätsurteile auch ohne gezielte Studien zu fällen.

Je besser die Erfassung extensiver kognitiver Prozesse gelingt, je mehr Befunde aussagekräftiger Studien vorliegen, desto Erfolg versprechender werden jene Versuche sein, diese kognitiven Prozesse nicht nur zu beschreiben, sondern auch zu erklären und zu prognostizieren. Die dabei zu erwartende Weiterentwicklung der Theorien des Käuferverhaltens wird dann auch besser zeigen können, wo jene 
Versuche anzusetzen sind, die das Kauferleben und das Kaufverhalten am POS beeinflussen bzw. steuern wollen.

\section{Literatur}

Büttner, O. (2008).

Die Untersuchung einkaufsbegleitender kognitiver Prozesse am Point of Sale. Eine Arbeit zur Qualität von Datenerhebungsmethoden der Konsumentenforschung, Dissertationsschrift, Universität Göttingen, Wirtschaftswissenschaftliche Fakultät

Büttner, O. \& Silberer, G. (2008).

"Video-cued thought protocols: A method for tracing cognitive processes at the point of purchase, in: Lowry, T. (Ed.), Brick \& Mortar Shopping in the 21st Century, Mahwah, NJ: Lawrence Erlbaum, pp. 221-241

Downs, R. M. \& Stea, D. (1973).

Cognitive Maps and Spatial Behaviour: Process and Products, in: R. M. Downs \& D. Stea (Eds.). Image and Environment, Chicago, Il.: Aldine Publishing Company, pp. $8-26$

Ericsson, K. A. \& Simon, H. A. (1980).

Verbal Reports as Data, in: Psychological Review, Vol. 87 (May 1980), No 3, pp. $215-251$

Fidler, E. J. (1983).

The Reliability and Validity of Concurrent, Retrospective, and Interpretive Verbal Reports: An Experimental Study, in: P. Humphreys, O. Svenson \& A. Vari (Eds.), Advances in Psychology, Vol. 14, Analyzing and Aiding Decision Processes, Amsterdam: North-Holland, pp. 429-40

Genest, M. \& Turk, D. C. (1981).

Think-About Approaches to Cognitive Assessment, in: T. V. Merluzzi, C. R. Glass \& M. Crewst (Eds.), Cognitive Assessment, New York: The Guilford Press, pp. $233-269$

McIntyre, S. H. \& Bender, S. D. F. G. (1986).

The Purchase Intercept Technique (PIT) in Comparison to Telephone and Mail Surveys, in: Journal of Retailing, Vol. 62 (Winter 1986), No 4, pp. 364-383

Nisbett, R. E. \& Wilson, T. D. (1977).

Telling more than we can know: Verbal Reports on Mental Processes, in: Psychological Review, Vol. 84 (May 1977), No 3, pp. 231-259 
Passini, R. (1984).

Wayfinding in architecture, New York-Cincinnati-Toronto etc.: Van Nostrand Reinhold Company

Payne, J.W. \& Ragsdale, E. K. E. (1978).

Verbal protocols and direct observation of supermarket shopping behaviour: Some findings and a discussion of methods, in: H. K. Hunt (Ed.), Advances in Consumer Research, Chicago: Association for Consumer Research, Vol. 5, pp. 571-577

Reicks, M., Smith, C., Henry, H., Renier, K., Atwell, J. \& Thomas, R. (2003).

Use of the Think Aloud Method to Examine Fruit and Vegetable Purchasing Behaviours among Low-Income African American Women, in: Journal of Nutrition Education and Behaviour, Vol. 35 (May 2003), No 3, pp. 154-163

Russo, J. E., Johnson, E. J. \& Stephens, D. L. (1989).

The validity of verbal protocols, in: Memory \& Cognition, Vol. 17 (1989), No 6, pp. 759-769

Silberer, G. (2005).

Die videogestützte Rekonstruktion kognitiver Prozesse beim Ladenbesuch, in: Marketing. ZFP, Vol. 27 (2005), Heft 4 , S. 263-271

Silberer, G. \& Büttner O. (2008).

Marktforschung am Point of Sale, in: A. Herrmann, C. Homburg \& M. Klarmann (Hg.). Handbuch Marktforschung, 3. Aufl., Wiesbaden: Gabler, S. 1097-1123

Titus, Ph. A. \& Everett, P. B. (1996).

Consumer Wayfinding Tests, Strategies and Errors: An Exlaboratory Field Study, in: Psychology \& Marketing, Vol. 13 (1966), No 3, pp. 265-290

Wallendorf, M. \& Brucks, M. (1993).

Introspection in Consumer Research: Implementation and Implications, in: Journal of Consumer Research, Vol. 20 (Dec. 1993), pp. 339-359

Weidle, R. \& Wagner, A. C. (1982).

Die Methode des Lauten Denkens, in: G. L. Huber \& H. Mandl (Hg.), Verbale Daten, Weinheim: Beltz, S. 80-103 
D

Die Kaufbegleitung als Forschungsmethode 


\section{Einleitung}

Seit geraumer Zeit versucht die Käuferforschung, das Verhalten der Verbraucher beim Kaufen von Verbrauchs- und Gebrauchsgütern, aber auch von Dienstleistungen, zu beschreiben und zu erklären. Fortschritte betreffen nicht nur die Theorien, sondern auch die Forschungsmethoden. Dabei gilt, dass Fortschritte bei den Forschungsmethoden den Fortschritt in der Theoriebildung unterstützen. Deshalb verdienen die Forschungsmethoden dieselbe Aufmerksamkeit wie die Theorien, wenn der Erkenntnisfortschritt fortschreiten soll.

Eine neue Forschungsmethode, die bereits vor einigen Jahren entwickelt und beschrieben wurde, ist die Kaufbegleitung (Alexis et al. 1968, King 1969, Bettman \& Zins 1977, Iyer 1989, Park et al. 1989, Otnes et al. 1995, Lowrey et al. 2005). Die dabei propagierte Kaufbegleitung, häufig „Shopping With Consumers“ (SWC) genannt, beruht auf der Überzeugung, dass sich das Verständnis in Sachen Kaufverhalten vor allem dadurch generieren bzw. verbessern lässt, dass der Forscher den Käufer beim Einkaufen begleitet. Aus diesem Grunde ist der Ausdruck „Kaufbegleitungsmethode“ (KBM) viel treffender, weil die Kaufbegleitung nicht einkaufen geht, sondern den Einkaufenden lediglich begleitet.

Näher betrachtet geht es bei der Kaufbegleitungsmethode nicht nur um die Begleitung, sondern auch um das Vorgespräch und um das Nachgespräch, auch um wiederholte Kaufbegleitungen. Auf diese Weise lassen sich vor allem Hinweise auf die Motive und auf Gewohnheiten des Käufers gewinnen (Otnes et al. 1997, Lowrey et al. 1998 S. 179). Das Vorgehen sei zwar aufwendig und für große Stichproben nicht geeignet, dennoch würde sich der im Vergleich zu klassischen Befragungs- und Beobachtungsmethoden durchaus beachtliche Aufwand lohnen, zum einen, weil sich zwischen Forscher und Käufer ein Vertrauensverhältnis aufbauen könne, zum anderen, weil der Forscher nicht nur zuschauen und zuhören könne, sondern sich auch mehr über die Lebenswelt des Käufers in Erfahrung bringen ließe. 
Die Beurteilung der Kaufbegleitungsmethode kennt aber auch kritische Stimmen. Diese verweisen nicht nur auf den hohen Aufwand, sondern auch darauf, dass der Forscher auf das Verhalten des Käufers Einfluss nehme, dass sich der Forschungsvorgang schlecht reproduzieren lasse und der Forscher nicht mehr als objektiv - objektiv im Sinne von „distanziert“ - bleiben könne (Otnes et al. 1995 S. 104f.). Eine Beurteilung der Kaufbegleitungsmethode sollte bei den bislang geäußerten Urteilen nicht stehen bleiben, sondern die Ausgestaltung dieser Methode und die verschiedenen Varianten näher betrachten und differenziertere Urteile fällen. Viele Varianten sind noch gar nicht bedacht, geschweige denn in ihrem Wert für die künftige Käuferforschung evaluiert worden.

In dieser Situation ist es jedenfalls geboten, sich mit der KBM etwas näher zu befassen. Wir wollen uns in diesem Beitrag mit folgenden Fragen auseinandersetzen: Welche Varianten der Kaufbegleitungsmethode lassen sich unterscheiden? Welche Sachverhalte lassen sich mit welchen Varianten mehr oder weniger gut erfassen? Und wie könnte und sollte sich der künftige Einsatz der Kaufbegleitungsmethode entwickeln?

\section{Grundlegende Arten der Kaufbegleitung}

Die Kaufbegleitung ist ein alltägliches Phänomen. Paare, Bekannte oder Verwandte gehen nicht selten miteinander einkaufen. Eine solche Kaufbegleitung kann geplant und verabredet sein, kann sich aber auch ganz spontan aus der Situation heraus ergeben. Als Forschungsmethode ist die Kaufbegleitung jedoch alles andere als ein alltägliches Phänomen. Der Forscher gehört nicht zum Kreis der Familie, der Verwandten oder Bekannten. Dennoch möchte er beim Einkauf mitgenommen werden bzw. mitgehen dürfen, um Einsichten in das Einkaufsverhalten des Probanden zu gewinnen. Er möchte nicht nur als Begleiter und Beobachter, sondern auch als Gesprächspartner akzeptiert werden, der im Vorgespräch etwas über die Absichten und die Geschäftswahl erfahren will und im Nachgespräch die Gelegenheit erhalten möchte, Fragen nach dem „Warum“ und dem „Wozu“ zu stellen. 
Angesichts dieser Situation ließe sich fragen, ob nicht auch natürliche Begleitpersonen beauftragt werden könnten, Aufgaben der Forschung zu übernehmen und hinterher von ihren Erkenntnissen zu berichten. Ein solches Vorgehen würde vermutlich weit weniger Aufwand verursachen. Dennoch wäre zu bedenken, dass die Zielperson nach wie vor frei wäre, über die Kaufbegleitung jederzeit neu zu entscheiden, und dass die natürliche Begleitung keinesfalls objektiv und ggf. gut eingewiesen bzw. ausgebildet sein muss.

Im Folgenden interessiert und beschäftigt uns die Kaufbegleitung allein als Begleitung ausgewählter Probanden durch den Forscher als Drittperson. Damit sei nicht in Abrede gestellt, dass eine Befragung natürlicher Begleiter der Zielpersonen ebenfalls sinnvoll sein kann.

\subsection{Die Kaufbegleitung als ethnographienahe Methode}

Die Überzeugung, dass ein Forscher mehr über das Verhalten von Menschen in Erfahrung bringen kann, wenn er sich ihnen intensiv widmet, sie in ihrem Alltag begleitet, mit ihnen über alles Mögliche spricht und zugleich ein Vertrauensverhältnis aufbaut, erinnert an die Ethnographie (s. z.B. Hitzler 2007, Johnson \& Bolstadt 1973).

Die Ethnographie steht - grob gesprochen - für ein Vorgehen, bei dem das Interesse am gelebten Alltag und das Eintauchen in die Lebenswelt der Probanden eine zentrale Rolle spielen (Müller 1972, 1980, Hammersley \& Atkinson 1983, Barnard 2000, Eriksen 2001, Kuper 2002). Kennzeichnend ist dabei auch das Bemühen um eine Innenperspektive, das Sich-Einfühlen in den Untersuchungsgegenstand, auch das Sich-Anpassen an die jeweils vorherrschende Kultur, vor allem Vertrautheit im Umgang mit den untersuchten Menschen. Kennzeichnend ist für dieses Vorgehen außerdem der Zugang zu Sachverhalten, die sich in Worten nur schwer ausdrücken lassen. Hieraus erwächst auch die große Bedeutung einer anschaulichen Dokumentation anhand von Erzählungen sowie von Aufnahmen in Wort und Bild (vgl. Otnes et al. 1995 S. 102-104, Lowrey et al. 2005 S. 176). 
Die Kaufbegleitung ist jedoch viel weniger als das Eintauchen in eine ganze Lebenswelt. Sie stellt von daher gesehen keine Ethnographie i.e.S. des Wortes dar. Dennoch kann von einer Nähe zur Ethnographie gesprochen werden, wenn eine ggf. wiederholte Kaufbegleitung mit ausführlichen Gesprächen, ggf. im Haushalt der einkaufenden Person, auch dazu dient, die Lebenswelt der einkaufenden Personen und ihrer Familie, deren Kauf- und Konsumgewohnheiten sowie deren Werten und Normen in Erfahrung zubringen und zu dokumentieren. Eine umfassende und anschauliche Dokumentation des Einkaufens, auch der Rahmenbedingungen des Einkaufens, könnte es dem Außenstehenden in der Tat leicht machen, sich vom Einkaufsverhalten einer Person oder einer Familie „ein Bild zu machen" und das Einkaufsverhalten in seiner lebensweltlichen Einbettung zu „verstehen“.

So aufschlussreich eine Kaufbegleitung mit Nähe zur Ethnographie auch sein kann, die entsprechenden Einsichten haben ihren Preis. Der Aufwand an Zeit und gut ausgebildetem Personal ist hoch, es sei denn, dass man sich dabei mit extrem kleinen Stichproben begnügt, die dann allerdings kaum noch Stichprobencharakter i.e.S. aufweisen. Deshalb stellt sich die Frage, ob der sicherlich sinnvolle ethnographische Charakter immer so stark ausgeprägt sein muss. Unsere Antwort lautet: Nein. Denn: Eine Kaufbegleitung kann auch im Sinne einer klassischen, d.h. einer „verstärkt standardisierten und leichter zu replizierenden Verhaltensforschung" verstanden, ausgestaltet und umgesetzt werden.

\subsection{Die Kaufbegleitung als klassische Verhaltensforschung}

Wenn es darum geht, die Möglichkeiten einer Kaufbegleitung im Sinne einer stärker standardisierten, leichter zu replizierenden Verhaltensforschung aufzuzeigen, liegt es nahe, den Blick auf das relevante Verhalten zu richten.

Das Einkaufsverhalten besteht aus verschiedenen Abschnitten. Es beginnt mit der Planung und der Vorbereitung des Einkaufs. Zur Planung gehört die Bedarfsanalyse, die Erstellung einer realen oder einer kognitiven (virtuellen) Einkaufsliste und die Zeitplanung. Bei der Vorbereitung des Einkaufs kann es z.B. 
darum gehen, Leergut einzusammeln, Geldmittel zu besorgen und Transporthilfen bereit zu stellen. Dann folgt das Aufsuchen des (der) relevanten Geschäfts(e) bzw. die Anfahrt zum (zu den) ausgewählten Geschäft(en), ggf. verbunden mit der Suche einer Parkmöglichkeit und konkreten Nutzung von Parkmöglichkeiten. Der Geschäftsbesuch schließt sich an, ggf. auch die Nutzung gastronomischer Angebote und sonstiger Dienstleistungen, die in einem Einkaufszentrum oft mit angeboten werden. Im Geschäft kann es nicht nur ungeplanten Informationsaktivitäten (Xia 2003) kommen, sondern auch zu ungeplanten Käufen (Iyer 1989, Youn \& Faber 2000). Nach dem Einkauf will die Ware zum Auto gebracht, dort verstaut oder auf andere Weise nach Hause gebracht werden. Die letzte Phase besteht dann darin, die Ware zuhause auszupacken, zu verstauen, ggf. auch lagerfähig zu machen, und Transporthilfen an ihrem angestammten Platz zu verstauen. Vielleicht muss auch noch die Transportverpackung entsorgt werden. Damit wäre dann der Einkauf als eine Art Versorgungsvorgang abgeschlossen.

Die Hinweise auf verschiedene Abschnitte machen schon deutlich, dass das Kaufverhalten aus einem offenen, beobachtbaren Verhalten, aber auch aus einem inneren, verdeckten, nicht unmittelbar beobachtbaren Verhalten besteht. Beobachtbar ist z.B. das Anreise- und Abreiseverhalten, die Rückgabe von Leergut, das Lauf- und Zuwendungsverhalten in den Verkaufszonen, das Einpacken und das Bezahlen der Ware und Aufenthalte in der Gastronomie und in Ruhezonen. Zu den nicht beobachtbaren Verhaltensweisen zählen u.a. das Entwickeln von Kaufplänen bzw. Kaufabsichten, das Orientierungsverhalten im Geschäft, das Betrachten des Angebots, die Auswahl von Produkten und Marken. Zu erwähnen sind auch ungeplante Kaufentscheidungen, wenn günstige, attraktive Angebote ohne langes Überlegen mitgenommen werden (Impulskäufe), wenn Angebote und Informationen einen überlegten Kauf auslösen (Überzeugungskäufe) und wieder andere daran erinnern, dass hier schon seit längerer Zeit ein Kauf beabsichtigt war (Erinnerungskauf). 
Vor dem Hintergrund der aufgelisteten Phasen und der beiden Verhaltensebenen wird zweierlei deutlich: Zum einen die Notwendigkeit, Methoden der Beobachtung und der Befragung miteinander zu verbinden, was bei der KBM auch tatsächlich der Fall ist. Zum anderen die Sinnhaftigkeit, ein dreistufiges Vorgehen zu wählen, nämlich den Einstieg mit einem Vorgespräch, dann die Käuferbegleitung im Geschäft und schließlich das Nachgespräch (zu den Phasen einer Kaufbegleitung s. Bettman 1970 S. 371f., Otnes et al. 1995 S. 106f., Koch 1997 S. 86 sowie Lowrey et al. 2005 S. 177-179). Auch dieses Vorgehen ist für die KBM charakteristisch. Wie die KBM im Einzelfall auszugestalten ist, ergibt sich aus den Umständen und aus den Erkenntniszielen. Grundsätzlich kann dabei das ganze, reichhaltige Arsenal der Verhaltensforschung zum Einsatz kommen, von den Beobachtungsmethoden über Registriermethoden bis hin $\mathrm{zu}$ den Befragungsmethoden, die Denke-Laut-Methode und die Möglichkeiten einer gestützten Gedankenrekonstruktion inklusive.

\section{Die Kaufbegleitung als Methoden-Mix}

Die Kaufbegleitung ist nicht nur insgesamt gesehen ein Methoden-Mix, auch in den einzelnen Phasen der Kaufbegleitung können jeweils mehrere Methoden zum Einsatz kommen. Im Folgenden werden ausgewählte Möglichkeiten einer Methodenkombination aufgezeigt, und zwar für die Vorkaufsphase, für die Einkaufsphase und für die Zeit nach dem Einkauf.

\subsection{Der Methodeneinsatz in der Vorkaufphase}

In der Vorbereitung einer Kaufbegleitung kann es zwei Kontakte geben: den einen im Rahmen der Teilnehmerakquise, den anderen beim eigentlichen Vorgespräch, das ein strukturiertes (Vorkauf-)Interview, aber auch erste Beobachtungen mit einschließen kann. 


\section{(1) Das Methoden-Mix in der Teilnehmerakquise}

Wenn es darum geht, Teilnehmer zu finden und ein Teilnahmeversprechen zu erwirken, gibt es mehrere Möglichkeiten: Die Ansprache mit dem Ziel, eine Kaufbegleitung zu einem späteren Zeitpunkt zu verabreden („VerabredungsAkquise") und die Bitte um Zustimmung zur sofortigen Begleitung am Eingang eines Geschäfts („Kaltakquise“).

Die Verabredungs-Akquise kann über Anzeigen mit einem Aufruf zur Teilnahme, über die Ansprache im WWW mit sofortiger Antwortmöglichkeit, über die persönliche Ansprache im Verwandten- und Bekanntenkreis, die Ansprache im Hörsaal und auch über die Ansprache vor Geschäften erfolgen, die für eine Kaufbegleitung zu einem späteren Zeitpunkt in Frage kommen (Alexis et al. 1968 S. 198, Bettman \& Zins 1977, Park et al. 1989, Otnes et al. 1995, McGrath \& Otnes 1995, Lowrey et al. 1998 S. 310). Bei diesem Vorgehen liegt es nahe, Namen, Adressen und Telefonnummern oder eMail-Adressen beider Seiten auszutauschen, damit der Zeitpunkt und der Ort der anvisierten Kaufbegleitung noch einmal oder gar zum ersten Mal abgestimmt werden können, ggf. auch geändert werden können. Werden auch Angaben zur Person festgehalten, lassen sich bei späteren Absagen die Teilnehmer mit den Nichtteilnehmern vergleichen und somit Selektivitätseffekte bestimmen. Bei der Verabredungs-Akquise liegt es auch nahe, bestimmte Kaufgewohnheiten, z.B. häufig besuchte Geschäfte und präferierte Wochentage oder günstige Tageszeiten in Erfahrung zu bringen. Findet die Verabredungs-Aquise am Ausgang eines Geschäfts statt, ließe sich auch der Einkaufskorb erfragen oder einsehen. Entsprechende Feststellungen könnten ggf. sogar als Auswahlkriterium oder als Vorgabe bezüglich der geplanten Kaufbegleitungs-Stichprobe herangezogen werden.

Bei der Kaltakquise werden Ladenbesucher vor Ort mit der Bitte angesprochen, einer Kaufbegleitung zuzustimmen. Dabei ist die Gefahr, dass die Angesprochenen keine Zeit für das Vor- und das Nachgespräch haben, etwas 
größer, dafür entfällt der Aufwand, der mit einer Verabredungs-Akquise verbunden ist und auch dann entsteht, wenn die Verabredung gar nicht eingehalten wird.

Die kurzen, informellen Gespräche im Rahmen der Teilnehmerakquise können auch dazu dienen, den Sinn und Zweck der Kaufbegleitung kurz zu erläutern, Nachfragen zu beantworten und sog. Belohnungen (Anreize), die zur Teilnahmebereitschaft führen sollen, zu versprechen. So können z.B. Geldbeträge als Teilnehmerhonorare angeboten werden, aber auch Gutscheine, die sich in relevanten Geschäften einlösen lassen.

\section{(2) Das eigentliche Vorgespräch (Vorab-Interview)}

Das „eigentliche Vorgespräch“ dient der gezielten Abfrage von Sachverhalten, die im Rahmen der Kaufbegleitung erforscht werden sollen. Es handelt sich somit eher um ein Interview. Dabei können z.B. die Gründe für die Geschäftswahl, die Kaufpläne, die Vorbereitung des Kaufs, die „Anreise“ und die verfügbare Zeit abgefragt werden. Weiß der Proband, welche Areale er aufsuchen will, kann auch dies festgehalten werden. Von Interesse könnten auch das verfügbare Budget, die geplante Ausgabensumme und die präferierte Bezahlweise sein.

Das Vorab-Interview findet kurz vor dem Betreten des Geschäfts statt und sollte deshalb nicht allzu viel Zeit in Anspruch nehmen. Als Ort kommt somit die Eingangszone in Frage. Wird ein Proband jedoch von zuhause abgeholt, kann die Vorab-Befragung auch im Domizil des Probanden oder während der Anfahrt - im Auto oder im öffentlichen Verkehrsmittel - stattfinden. Handelt es sich um ein vereinbartes Treffen und ist der Proband aller Voraussicht nach in Eile, könnte das Vorab-Interview auch schon bei der Vereinbarung z.B. per Telefon oder per Online-Befragung geführt werden.

\section{(3) Beobachtungen vor dem Kauf}

Im Vorgespräch eröffnen sich auch Möglichkeiten, einige Dinge zu beobachten und zu registrieren. Dazu zählen das Outfit des Probanden, dessen Verhalten und 
ggf. auch dessen Einkaufsliste. Wird er zuhause abgeholt, kann sich die Möglichkeit ergeben, auch einige Aspekte der Lebenssituation und des Lebensstils zu erkunden. Wird der Proband bei seinem Einkauf von anderen Personen begleitet, können auch diese nicht nur befragt, sondern auch beobachtet werden.

\subsection{Der Methodeneinsatz bei der In-Store-Begleitung}

Für die Begleitung beim Ladenbesuch macht es einen großen Unterschied, ob nur das Lauf- und Zuwendungsverhalten zu registrieren ist, oder ob auch oder allein zu erfassen ist, was dem Probanden durch den Kopf geht, worüber er denkt, was er sucht, wofür und wogegen er sich entscheidet.

\section{(1) Zur Erfassung des Lauf- und Zuwendungsverhaltens}

Das Laufverhalten betrifft den Weg, den der Ladenbesucher durchschreitet. Dabei kann es genügen, die Abfolge der durchschrittenen Verkaufs- und Ruhezonen festzuhalten. Zum Zuwendungsverhalten zählt vor allem die visuelle Zuwendung zu diversen Angeboten, das Anfassen, Zurücklegen und Mitnehmen von Waren, das Verkosten von Proben, die Konsultation von Mitarbeitern, das Gespräch mit der natürlichen Kaufbegleitung und dem kaufbegleitenden Forscher, das Verhalten an der Kasse und danach beim Einpacken und beim Abtransport.

Dieses Verhalten ist einer Beobachtung zumindest prinzipiell zugänglich. Bei der Kaufbegleitung liegt die teilnehmende Beobachtung auf der Hand. Der teilnehmende Beobachter ist aber eher passiv bzw. zurückhaltend. Er muss selbst nicht kaufen, muss auch keine Ratschläge erteilen, ggf. aber doch auf Fragen des Probanden antworten. Er ist jedenfalls gut beschäftigt, wenn er das Lauf- und Zuwendungsverhalten des Probanden beobachten und festhalten soll. In Frage kommen hier das Aufzeichnen und Aufschreiben auf Papier, die Einträge auf einem Tablet-PC und das Festhalten mit einem Diktaphon bzw. einem Voice Recorder (Otnes et al. 1992, S. 483, 1993 S. 230, 1995 S. 107, McGrath \& Otnes 1995 S. 262, Wells \& LoSciuto 1996, Titus \& Everett 1996, Lowrey et al. 1998 S. 311). Selbst das Festhalten per Videokamera, die ganz unauffällig eingesetzt werden 
kann, so dass sich andere Ladenbesucher nicht irritiert fühlen oder gar Einfluss nehmen, kommt in Frage (zur videogestützten Rekonstruktion des Verhaltens siehe Silberer 2005c, 2006a, 2006b, 2008, Agafonoff 2006, Silberer \& Büttner 2008). Videoaufzeichnungen können zwei Zwecken dienen: zum einen können sie eine Vollständigkeit sichern, wenn der Kaufbegleiter wichtige Dinge gar nicht anders festhalten kann; zum anderen können sie beim Nachgespräch als Erinnerungsstützen und als Anknüpfungen dienen, wenn der Käufer z.B. erläutern soll, worauf er geachtet hat, was er am Regal im Produktkontakt konkret getan hat und worüber er im Gespräch mit Begleitpersonen und mit dem Verkaufs- und Servicepersonal des Hauses gesprochen hat (zur Videographie als Forschungsmethode s. Belk \& Kozinets 2005, Silberer 2005c, 2006a, 2006b).

\section{(2) Zur Erfassung kognitiver Prozesse}

$\mathrm{Zu}$ den Gedanken, die einem Ladenbesucher durch den Kopf gehen können, zählen viele Sachverhalte, so z.B. das Erinnern an die Kaufpläne, die Auswahl der Produkte, deren Kauf geplant war, aber auch das Auswählen von Produkten, deren Kauf nicht geplant war, das Reflektieren (Bedenken) von eigenen Wünschen, das Erinnern von Wünschen oder Bedarfsanmeldungen aus dem Haushalt, das Bedenken einer möglichen Produktverwendung, das Beachten von Budgetgrenzen und das Entwickeln von Kaufplänen, die später einmal realisiert werden sollen.

So wichtig derartige Prozesse sind, so schwierig ist es, sie richtig und vollständig zu erfassen. Da sie leicht vergessen werden, bietet es sich an, sie möglichst zeitgleich zu erfassen. Deshalb kommt als klassisches Instrument die „Denke-Laut-Methode“ (DLM) in Frage (s. Alexis et al. 1968 S. 198, King 1969, Bettman 1970 S. 371f., Bettman \& Zins 1977 S. 78, Payne \& Ragsdale 1978 S. 572, Park et al. 1989 S. 425f., Iyer 1989 S. 45-47). Hier hat der Besucher alles auszusprechen, was ihm gerade durch den Kopf geht und was er gerade fühlt. Als Aufzeichnungsgerät kann ein Tonband bzw. ein Diktophon zum Einsatz kommen. Ein schnelles Mitschreiben des begleitenden Forschers dürfte schwierig sein. Soll 
das laute Denken nicht zu falschen Eindrücken bei anderen Ladenbesuchern führen, wäre daran zu denken, die Gedankenartikulation mit einem Handy aufzunehmen und so ein ganz normales Telefongespräch „vorzutäuschen“.

Da das laute Denken auf das Denken selbst zurückwirkt und damit Artefakte produziert, wurde vorgeschlagen, Videoaufnahmen zum Verhalten im Ladengschäft, die auch Gespräche und Hintergrundgeräusche wiedergeben, als Gedächtnisstütze zu verwenden. Im Rahmen eines Nachgesprächs ließe sich das Video vorführen und der Proband bitten, die jeweils gehegten Gedanken zu erinnern bzw. zu rekonstruieren (Silberer 1989, 2005a, 2005b, Silberer \& Büttner 2004, 2008, Büttner \& Silberer 2007, 2008). Bei dieser Methode der videogestützten Rekonstruktion von Gedankengängen beim Einkauf wird - im Gegensatz zur Ethnographie - die Visualisierung nicht als Ergebnis der Forschung angesehen, sondern als ein Forschungsinstrument eingesetzt.

\section{(3) Kapazitätsgrenzen und deren Überwindung}

Ein sorgfältiges, vollständiges Tracking des Lauf- und Zuwendungsverhaltens und der kognitiven Prozesse beim Ladenbesuch kann sehr schnell auf Grenzen stoßen bzw. die Kaufbegleitung überfordern. Deshalb sind zwei Optionen zu bedenken, die Entlastung bieten: zum einen die Reduzierung der Aufgaben, zum anderen die Aufstockung der Kapazitäten.

Eine Reduzierung der Aufgaben und eine Entlastung der Kaufbegleitung ließe sich in zweierlei Hinsicht realisieren: zum einen über das Abteilungs- oder RegalSampling, zum anderen durch die Auswahl bestimmter Verhaltenselemente. Beim Abteilungs- oder Regal-Sampling wird nicht der vollständige Ladenbesuch analysiert, sondern nur der Besuch ausgewählter Abteilungen und Regale, ggf. nur einer Abteilung oder nur eines Regals. Bei der Selektion bestimmter Verhaltenselemente konzentriert sich die Kaufbegleitung z.B. auf den Laufweg, auf Orientierungshilfen bei der Produktsuche, auf bestimmte Zuwendungsreaktionen wie die Konsultation von Servicemitarbeitern oder auf das Mitnehmen von 
Produkten, auf bestimmte Gedanken wie die Überlegungen bei einer Markenwahl oder die kognitiv-affektiven Reaktionen auf Beeinflussungsversuche seitens des Verkaufspersonals.

Die Aufstockung der Kapazitäten auf zwei Begleitpersonen würde es erlauben, dass der eine Begleiter sich auf das Erfragen bestimmter Sachverhalte wie z.B. der Erwartungen und Enttäuschungen oder auf das erbetene „laute Denken“ und die dabei wichtigen Aufnahmen konzentriert, der andere sich dagegen nur mit dem Beobachten und Aufzeichnen des Lauf- und Zuwendungsverhaltens, ggf. mit den unauffälligen Videoaufnahmen beschäftigt. Ein Aufstocken der Begleiterzahl bietet sich erst recht dann an, wenn mehrere Käufer, z.B. Paare, Familien und Gruppen zu begleiten sind.

\subsection{Der Methodeneinsatz beim Nachgespräch}

Das Gespräch, das mit dem Ladenbesucher unmittelbar nach dem Besuch geführt wird, muss mit einem Partner rechnen, der wenig Zeit hat oder ungeduldig werden könnte. Deshalb sollten die wichtigsten Dinge strukturiert und vorab erfragt werden. Sollte dann noch Zeit für ein offenes Gespräch bleiben, umso besser.

Für das unmittelbare Nachgespräch als Danach-Interview kommen vor allem folgende Fragen in Betracht (vgl. Iyer 1989 , Park et al. 1989 S. 426, Otnes et al. 1995 S. 107, McGrath \& Otnes 1995 S. 262):

- Fragen nach der Realisierung der geplanten Käufe und nach Gründen, falls diese nicht getätigt wurden,

- Fragen nach dem Laufverhalten, falls dies von der geplanten Laufstrecke abweicht,

- Fragen nach den Gründen für die ungeplanten Käufe,

- Fragen nach den Verbundbeziehungen im Warenkorb, ob der Bedarf, das OneStop-Shopping oder Verkaufsförderungsmaßnahmen dazu geführt haben, bestimmte Dinge zusammen zu kaufen,

- sowie Fragen nach der verausgabten Summe und der Abweichung von der geplanten Ausgabensumme. 
Zu denken wäre auch an die Erfassung sog. nachlaufender Indikatoren, so z.B. die Erfassung von Änderungen bezüglich der Ladenkenntnis, der Produktkenntnis, der Preiskenntnis und der Produktbeurteilungen, um auf diese Weise etwas in Bezug auf Wahrnehmungen beim Geschäftsbesuch in Erfahrung zu bringen. Dabei müsste allerdings ein Vorher-Nachher-Vergleich durchgeführt und bedacht werden, dass die Vorher-Messung den Ladenbesucher sensibilisieren und somit beeinflussen kann.

Nach dem Ladenbesuch hat der Begleiter nicht nur die Möglichkeit, Fragen zu stellen und Antworten sowie andere Aussagen zu protokollieren, sondern auch, Beobachtungen anzustellen und festzuhalten. Von Interesse könnten dabei der realisierte Warenkorb, die verausgabte Summe und die Ausgestaltung des Abtransports und die Verkehrsmittelwahl bei der Rückkehr nach Hause sein. Eine Kopie des Kassenbons könnte dabei ebenso hilfreich sein wie ein Warenkorbfoto, wenn es die eingekauften Waren wiedergeben kann.

\section{Zum derzeitigen Stand der Kaufbegleitungsforschung}

\section{(1) Untersuchungsgegenstände und Erkenntnisziele}

Die bislang durchgeführten Kaufbegleitungsstudien befassen sich mit recht unterschiedlichen Sachverhalten. Vier Themenbereiche haben besondere Beachtung gefunden: Motive und Hintergründe, Entscheidungsstrategien und kriterien, nicht geplante Einkäufe und soziale Interaktionen. Für Motive und Hintergründe interessierten sich Otnes et al. (1995) sowie Lowrey et al. (2005). Otnes \& Lowrey (1993) und Otnes (1998) analysierten die Bedeutung von Brautmodengeschäften für Frauen, Otnes (1998) außerdem die Vorbereitung einer Hochzeitszeremonie. Mit Entscheidungsstrategien und -kriterien befassten sich die Studie von Alexis et al. (1968), vor allem für die Rolle des Preises beim Kauf von Kleidungsstücken, die Studie von King (1969), vor allem für die Gedanken beim Einkaufen, und die Studien von Bettman (1970) und Bettman \& Zins (1977), vor allem für Entscheidungsstrategien. Ungeplante Käufe standen im Vordergrund bei Park et al. (1989) und Iyer (1989), auch für die Rolle der Ladenkenntnis und des 
Zeitdrucks bei solchen Käufen. Die soziale Interaktion stand bei Otnes et al. (1992), Otnes et al. (2003) und Lowrey \& Otnes (2004) und im Vordergrund. Hier interessierte z.B. die Beziehung zwischen der kaufenden und der beschenkten Person. McGrath \& Otnes (1995) untersuchten die Interaktion zwischen den begleiteten Ladenbesuchern und anderen Ladenbesuchern.

Die bislang durchgeführten Kaufbegleitungsstudien zeichnen sich in ihrer großen Mehrheit dadurch aus, dass sie das Einkaufsverhalten näher und vor allem detaillierter beschreiben wollen als klassische Befragungs- und Beobachtungsstudien. Auch wenn die Hintergründe immer wieder zur Sprache kommen, so dominiert doch der deskriptive Charakter dieser Forschung. Darüber kann und sollte der von den Forschern oft gegebene Hinweis, dass sie mit ihrer Kaufbegleitung anschauliche Befunde und wertvolle Erkenntnisse zutage fördern konnten, nicht hinwegtäuschen. Studien, die dem Test konkreter, aus relevanten Theorien abgeleiteten Hypothesen dienen, sind ausgesprochen selten.

\section{(2) Untersuchungsdesigns und Stichprobengrößen}

Das Grundmuster einer Kaufbegleitungsstudie „Vorgespräch - Kaufbegleitung Nachgespräch" hat sich in der bisherigen Forschung bewährt. Dennoch entsprechen nicht alle Studien diesem Schema. Manchmal wird auf das Vorgespräch und manchmal auf das Nachgespräch verzichtet. Zu den Studien mit einem Vorgespräch zählen Iyer (1989), Otnes et al. (1993, 1995), McGrath \& Otnes (1995) und Lowrey et al. (1998, 2005), zu den Studien mit einem Nachgespräch Iyer (1989), Park et al. (1989), Otnes et al. (1995), McGrath \& Otnes (1995) und Lowrey et al. (2005). Manche Forscher praktizieren auch die Unterbrechermethode, um noch während des Einkaufens Fragen stellen zu können, so z.B. bei Sherry (1998) zur Reaktion des Ladenbesuchers auf aktuelle Sonderangebote. Alexis et al. (1968) begleiteten ihre Versuchpersonen beim Kauf von Kleidungsstücken zweimal hintereinander, Lowrey et al. (2004) in ihrer Langzeitstudie des Öfteren. 
Der ethnographische Charakter einiger Studien zeigt sich nicht nur im teilnehmenden Beobachten und in informellen Gesprächen, die Aufschlüsse über das alltägliche Leben und den Lebensstil als Hintergründe des Einkaufens erlauben, sondern auch an den kleinen Stichproben, die durch den Aufwand der einzelnen Fallstudien bedingt sind. Alexis et al. (1968) konzentrierten sich auf zwei, King (1969) auf vier, Lowrey et al. (2004) auf fünf, Lowrey et al. (1998) auf neun und Otnes et al. (1992) auf fünfzehn Probanden. Doch es gibt auch Studien mit größeren Stichproben, so z.B. die Untersuchung von Miller (1998), der 76 Hausfrauen im Norden Londons beim Einkaufen begleitete und dabei auch befragte.

\section{(3) Das Methoden-Mix}

Die bisherigen Studien zeigen auch eine Vielfalt im Methoden-Mix. Die Kombination der Befragung bzw. des Gesprächs mit der Beobachtung kennt nicht nur Varianten, sondern auch Ergänzungen durch andere Methoden. Einige Beispiele sollen dies verdeutlichen.

Iyer (1989) führte das Vorabgespräch bei den Probanden zuhause durch. Dabei interessierten ihn Kochpläne und Einkaufspläne. Außerdem wurde ein Termin für die Kaufbegleitung vereinbart. Die dann realisierte Kaufbegleitung begann mit dem Abholen des Probanden in dessen Domizil, dann folgte das diskrete Nachfolgen im Laden, bei dem auf das Zurücklaufen und das Prüfen von Produkten besonders geachtet wurde, und endete im Vergleich der geplanten und der realisierten Käufe. Die Nachbefragung befasste sich außerdem mit der Ladenvertrautheit, der Besuchshäufigkeit und den Gründen für die ungeplanten Käufe. Außerdem mussten die Probanden wieder zurück nach Hause gebracht werden.

Otnes, Lowrey \& Shrum (1997) wählten bei ihrer Studie zur Vorbereitung einer Hochzeit folgendes Vorgehen: Beginn mit getrennten Gruppendiskussionen, und zwar mit der Gruppe der Bräute und der Gruppe der Verlobten, dann Tiefeninterviews mit ausgewählten Bräuten, den aktiven Planern einer Hochzeit, 
dann die Kaufbegleitung bei diesen Bräuten und schließlich eine Nachstudie mit vier anderen Bräuten (S. 83). Die Nachstudie bei jenen Bräuten, mit denen kein Tiefeninterview durchgeführt wurde und die beim Einkaufen nicht begleitet wurden, erlaubte es, den Einfluss des Tiefeninterviews und der Kaufbegleitung zumindest einer ersten groben Einschätzung zu unterziehen.

Jackson et al. (2006 S. 50-52) wollten in ihrer dreijährigen Studie herausfinden, wie der Wandel im Lebensmitteleinzelhandel vom Verbraucher erlebt wird und wie er sich auf den Konsumenten und seinen Haushalt auswirkt. Um dies in Erfahrung zu bringen, kombinierten sie die Kaufbegleitung mit Gruppendiskussionen, Tagebuchaufzeichnungen und Hausbesuchen (inklusive Küchenbesichtigung). Dabei gewannen sie die Erkenntnis, dass Kaufentscheidungen nicht nur Qualitäts-, Geschmacks- und Werteinschätzungen beinhalten, sondern auch Aspekte wie Zugänglichkeit, Bequemlichkeit und Preiswürdigkeit. Es zeigte sich auch, dass diese Aspekte in ihrer Bedeutung mit den Lebensbedingungen im jeweiligen Haushalt variieren und dass der Zusammenhang zwischen der Wahl eines Geschäfts und dem Verhalten im Geschäft einerseits und den Bedingungen im Haushalt, z.B. den Gewohnheiten und komplexen Alltagsbedingungen, andererseits beachtet werden sollte (S. 54-58).

\section{(4) Zur Evaluation der Kaufbegleitungsmethode}

Die bislang vorgebrachten Hinweise auf die Eignung der Kaufbegleitungsmethode konzentrieren sich auf die Ergiebigkeit entsprechender Studien und auf die Selektivität der Stichproben, die begrenzte Objektivität der begleitenden Forscher und die Reaktivität der begleiteten Probanden. Die Protagonisten der Kaufbegleitungsmethode kennen diese kritischen Hinweise, stellen diesen aber immer den großen Nutzen gegenüber, der aus der Aussagekraft der Beobachtungsund Gesprächsprotokolle resultiere. Überzeugte Autoren und Anwender dieser Methode wie Otnes et al. (1995) und Lowrey et al. (2005) sprechen von der Reichhaltigkeit („richness") und Vielfalt („,variety“) der Erkenntnisse. In ihrer 
Formulierung kommt zum Ausdruck, dass es sich in erster Linie um eine Überzeugung handelt: „We believe that the variety of text generated while shopping with consumers is the biggest advantage of the method." (Lowrey et al. 2005 S. 180)

Solche Einschätzungen der Kaufbegleitungsmethode spiegeln eine für die Ethnographie kennzeichnende Überzeugung wider, eine Überzeugung, die nicht zuletzt auch den hohen Aufwand bei der Feldarbeit und der Dokumentation von Beobachtungen und Gesprächen rechtfertigt (zum Aufwand s. Otnes et al. 1995 S. 104f.). Entsprechende Urteile erklären sich aber auch daraus, dass sich viele Ethnographen dem heuristischen Ziel des „Verstehens“ viel stärker verpflichtet fühlen als der Aufgabe, konkrete Hypothesen zu entwickeln und einem strengen, reproduzierbaren Test zu unterwerfen.

\section{Zur künftigen Ausrichtung der Kaufbegleitungsforschung}

Das Erkenntnispotenzial der Kaufbegleitungsforschung lässt sich künftig steigern, indem (1) das Einkaufsverhalten in all seinen Phasen und all seinen Dimensionen anhand größerer Stichproben reproduzierbar erfasst wird, (2) das deskriptive, explorative Vorgehen verstärkt durch eine gezielte, theoriegeleitete Ursachenanalyse ergänzt und auf das Ziel der ,guten Theorie des Einkaufsverhaltens“ ausgerichtet wird, und (3) die Methodik der Kaufbegleitung als Instrumentenbündel selbst zum Gegenstand der Forschung avanciert, vor allem die externe und interne Validität der Kaufbegleitung.

\section{(1) Zur systematischen und reproduzierbaren Erfassung des Einkaufs- verhaltens}

Bislang durchgeführte Kaufbegleitungsstudien konzentrieren sich auf das Verhalten im Ladengeschäft. Deshalb sollte künftig auch das Verhalten vor und nach dem Ladenbesuch eine stärkere Aufmerksamkeit erfahren. Dies könnte dazu führen, dass vor dem Ladenbesuch zwei Vorgespräche und nach dem Ladenbesuch zwei Nachgespräche erforderlich werden. Dem Mehraufwand stünde dann die 
Möglichkeit gegenüber, das Verhalten im Laden noch besser zu verstehen, wenn alle Phasen des Einkaufsgeschehens ins Blickfeld rücken.

Aber auch das In-Store-Verhalten bedarf einer genaueren, reproduzierbaren Analyse, vor allem über die gezielte Erfassung des offenen und des inneren Verhaltens. Hier sollten vor allem die Dynamik des Verhaltens und das Zusammenspiel des Lauf- und Zuwendungsverhaltens einerseits und der kognitiven Prozesse andererseits interessieren. Der Einsatz moderner Video- und Tontechnik bietet nicht nur gute Tracking-Möglichkeiten, sondern kann auch helfen, die geeigneten Erinnerungs- und Interpretationshilfen zu produzieren.

Eine große Herausforderung für künftige Kaufbegleitungsstudien besteht darin, nicht nur einzelne Ladenbesucher, sondern auch Pärchen, Familien sowie Gruppen beim Ladenbesuch zu begleiten und in ihrem individuellen Verhalten, aber auch in ihrer sozialen Interaktion zu studieren. Spätestens hier muss die Frage beantwortet werden, wann kaufbegleitende Forscher an ihre Grenzen stoßen und was getan werden kann, um deren Aufgabe an das Machbare und Vertretbare anzupassen.

Vieles lässt vermuten, dass die bisherige Kaufbegleitungsforschung vor allem Geschäftsbesucher mit Kaufabsicht vor Augen hatte. Vielleicht liegt dies daran, dass viele Studien in den USA durchgeführt wurden und dort der Ladenbesuch in ernsthafter Kaufabsicht dominiert. Dort und auch anderswo gibt es aber auch ein Besucherverhalten, das auf Information, Orientierung, Abwechslung, Entspannung oder gar nur Verkostung ausgerichtet ist und mit Freizeitgestaltung mehr zu tun hat als mit dem Einkaufen. Derartige Ladenbesuche sollten in der Kaufbegleitungsforschung einen höheren Stellenwert erhalten, zumal ein „browsing“ ungeplante Käufe gar nicht ausschließt.

Ein weiteres Augenmerk sollte die künftige Kaufbegleitungsforschung auf die Dokumentation des Verhaltens und der jeweiligen Ladenwelt und der jeweiligen Lebenswelt der Verbraucher richten. Dem Vorbild der Ethnographie entsprechend wäre es zu begrüßen, wenn sich künftige Forschungsberichte bemühten, das Anschauliche und Reichhaltige der Ergebnisse einer Kaufbegleitungsstudie zu 
vermitteln. Was eine Videographie zu Tage fördert, kann zwar nicht in allen Medien dargestellt werden, aber auf jeden Fall auf einer Website im Internet, auf deren Adresse sich dann doch in allen Medien verweisen lässt.

\section{(2) Zur theoriegeleiteten Ursachenanalyse, gezielte Hypothesentests inklusive}

Auf dem Weg zu einer guten Theorie des Einkaufsverhaltens bedarf es zunächst guter Ideen im sog. „Entstehungszusammenhang“. Hier kann auch jene Kaufbegleitungsforschung wertvolle Dienste leisten, die sich der ethnographischen Richtung verpflichtet fühlt. Auch einzelne Befunde, so z.B. die Dokumentation der „Lebenswelt" des Ladenbesuchers und der von ihm gewählten „Ladenwelt“, können wertvolle Anregungen für die Genese von Kausalhypothesen liefern.

Auf dem Weg zu einer guten Theorie des Einkaufsverhaltens bedarf es dann geeigneter Hypothesentests. Auch dabei kann die Kaufbegleitungsforschung mitarbeiten. Mehrere Ansatzpunkte sind zu unterscheiden: Erstens die Realisierung von experimentellen Designs, zumindest aber die Durchführung sog. Quasiexperimente. Hier wäre z.B. daran zu denken, dass das Einkaufsverhalten vor, während und nach einer Verkaufsförderungsaktion oder einem Ladenumbau untersucht wird. Zweitens die klare und reproduzierbare Operationalisierung relevanter Variablen bzw. theoretischer Konstrukte anhand geeigneter Messmodelle. Drittens die valide Messung dieser Variablen im Einsatz des geeigneten Methodenbündels. Und nicht zu vergessen: Die Wahl geeigneter Auswertungsmethoden, so z.B. der Einsatz der Sequenzanalyse bei der Auswertung von Abfolgen im Betreten verschiedener Flächen, von Abfolgen bei der Zuwendung zu verschiedenen Angeboten und bei Abfolgen in den sog. Gedankengängen. 


\section{(3) Zur Erforschung der internen und externen Validität einer}

\section{Kaufbegleitung}

Auch wenn es in der Kaufbegleitungsforschung bislang nicht gerade üblich war, die Frage nach der Aussagekraft der Ergebnisse im Lichte der klassischen Forschungslogik aufzuwerfen, so macht es doch Sinn, dies in Zukunft zu tun. Dies bedeutet, die relevanten Kriterien der internen und externen Validität zu bestimmen und die Kaufbegleitung dann entsprechend zu evaluieren. Bei der Beurteilung der internen Validität eines Messinstruments kennen wir zwei Kriterienklassen: zum einen die Reliabilitätskriterien als notwendige, aber nicht hinreichende Bedingungen, zum anderen die Validitätskriterien als notwendige und hinreichende Bedingungen. Werden erstere erfüllt, lohnt es sich, die letzteren in einem zweiten Schritt zu prüfen. Werden erstere nicht erfüllt, dann brauchen letztere nicht mehr geprüft werden. Dieses abgestufte Vorgehen ist dann ein ökonomisches Procedere, wenn die Validitätstests aufwendiger und schwieriger sind als die Reliabilitätstests.

Zu den klassischen Reliabilitätskriterien zählt die Objektivität, die Übereinstimmung von Test- und Nachtestergebnissen (Retestreliabilität) und die Korrelation von Messergebnissen an solchen Messpunkten, die alle dasselbe abzubilden vorgeben (Indikatorenkorrelation). Zu den klassischen Validitätskriterien zählen die Augenscheinvalidität, die am Urteil eines Experten festmacht, die Kriteriumsvalidität, die am Messergebnis eines geeigneten Prüfkriteriums festmacht, die Konvergenzvalidität, die sich aus dem Vergleich mit einem anderen, bewährten Messinstrument ergibt, und die nomologische Validität, die sich an den Aussagen einer guten Messtheorie orientiert. Kann die interne Validität und damit die Aussagekraft der Befunde vorausgesetzt werden, so ist noch zu klären, ob die Befunde auf größere Populationen und andere, vergleichbare, nicht untersuchte Einkaufsaktivitäten schließen lassen. Ist dies der Fall, so kann von einer Generalisierbarkeit bzw. von einer externen V alidität der Befunde gesprochen werden. 
Beim Verfahren der Kaufbegleitung kann als Reliabilitätstest am ehesten eine Prüfung der Objektivität in Betracht gezogen werden. Eine Kaufbegleitung müsste bei verschiedenen Begleitpersonen zum selben Ergebnis führen. Doch das Problem besteht darin, dass dabei Lerneffekte nicht ausgeschlossen werden können. Ähnliches gilt für Retestreliabilität. Eine Kaufbegleitung müsste sich beim Retest dadurch bewähren, dass derselbe Kaufbegleiter bei ein- und derselben Person bei identischen Kaufsituationen nacheinander zum selben Ergebnis käme. Ein Test der Indikatorenkorrelation setzt voraus, dass sich bestimmte Sachverhalte anhand verschiedener Indikatoren erfassen lassen. Dies kann bei bestimmten Sachverhalten durchaus der Fall sein, so z.B. bei der Erfassung von Entscheidungsstrategien und bei der Erfassung der Ladenatmosphäre. Die Itemkorrelation als Reliabilitätsmaß für die Erfassung des gesamten Einkaufsverhaltens macht dagegen keinen Sinn. Angesichts dieser Sachlage ist zu verstehen, dass in der Kaufbegleitungsforschung bislang keine Reliabilitätstests durchgeführt worden sind und dass auch für künftige Tests kaum Spielräume gegeben sind. Die Zweifel an der Objektivität sind jedenfalls auch in Zukunft ernst zu nehmen. Immerhin haben selbst überzeugte Protagonisten der SWC-Methode schon immer die Auffassung vertreten, dass die Ergiebigkeit dieser Methode nicht nur mit einem hohen Aufwand an Zeit und Personal, sondern auch mit einer Einbuße an Objektivität erkauft werden muss.

Wenn nun Lowrey et al. (1998 S. 313, 2005 S. 184) vorschlagen, bereits beim Abfassen des Ergebnisberichts andere Experten hinzuzuziehen und einen Konsens in Bezug auf die Formulierung der Resultate zu erarbeiten, kann dies die Objektivität der Ergebnisinterpretation durchaus fördern. Dies gilt vor allem dann, wenn auf diesem Wege die Erwartungen und Vorurteile der Kaufbegleiter identifiziert und dann auch abgebaut werden können.

In Bezug auf die interne Validität der Kaufbegleitungsmethode ist zunächst festzuhalten, dass wir derzeit weder über eine gute Messtheorie verfügen, die angibt, wann und worin sich die relevanten Sachverhalte zeigen (nomologische Validität), noch über bereits validierte, bewährte Messinstrumente (Konvergenzvalidität). 
Deshalb muss es in Zukunft darum gehen, diese beiden Lücken zu schließen. Für eine gewisse Augenscheinvalidität spricht der Umstand, dass sich Ergebnisse der Beobachtung und der Befragung gegenseitig ergänzen. Hierauf sollte in Zukunft besonderes Augenmerk gelegt werden. Einen anderen Weg zur Sicherung der internen Validität einer Kaufbegleitung benennen Lowrey et al. (2005 S. 183f.). Sie unterstreichen das Erfordernis, die Ausbildung der Kaufbegleiter, der Gesprächsleiter und der Interviewer umfassend und qualifiziert durchzuführen. Bleibt noch die Kriteriumsvalidität. Bei komplexen Sachverhalten wie dem Verhalten und Erleben beim Einkaufen müssten jedenfalls recht viele Kriterien zur Verfügung stehen, an denen sich gute Messungen der einzelnen Variablen festmachen lassen. Versuche, die Kriteriumsvalidität der KBM zu bestimmen, sind bislang nur bezüglich einzelner Variablen unternommen worden. So liefern Büttner \& Silberer (2008) Hinweise darauf, dass eine Videogestützte Gedankenrekonstruktion durchaus valide Einblicke in kognitive Prozesse geben kann. Weitere Versuche in dieser Richtung wären aufwendig, aber sicherlich recht sinnvoll.

Die Methodenforschung sollte in Zukunft neben der internen Validität der Kaufbegleitung auch die externe Validität stärker untersuchen. Dies bedeutet vor allem, dass geprüft wird, wann von den freiwilligen Probanden auf größere Populationen und vom analysierten Verhalten auf das Verhalten bei vergleichbaren, nicht untersuchten Einkäufen geschlossen werden kann.

Die KBM muss mit verschiedenen Selektivitätseffekten rechnen (vgl. Groves et al. 1992, 2000, 2004). Eine erste Selektivität kann aus der Versuchspersonenansprache resultieren. Dies wäre z.B. dann der Fall, wenn der Forscher nur Verwandte und Bekannte anspricht. Es könnte aber auch sein, dass bei einer Ansprache völlig unbekannter Verbraucher nach einer gewissen Zeit jene Personen überdurchschnittlich häufig angesprochen werden, bei denen eine überdurchschnittliche Teilnahmebereitschaft erwartet wird. Diese Selektivität lässt sich durch gezielte Maßnahmen leicht reduzieren. Eine zpeite Selektivität ergibt aus der ungleich 
verteilten Teilnahmebereitschaft bei den angesprochenen Probanden. Nicht alle sind zu einer Teilnahme bereit, sei es aus Zeitgründen, sei es aus anderen Gründen. Es können sowohl grundsätzliche Bedenken bestehen als auch die Ablehnung der Person oder Organisation, welche die Begleitungsstudie durchführen möchte. Solche Ablehnungen erzeugen dann eine Selektivität der Stichprobe, wenn sie sich nicht auf alle angesprochenen Personen gleich verteilen, wenn z.B. ältere Personen öfter ablehnen als jüngere, Singles weniger oft ablehnen als Paare. Diese zweite Selektivität lässt sich nicht vermeiden, aber durch geeignete Maßnahmen wie z.B. gezielte Anreize zumindest etwas reduzieren. Eine dritte Selektivität ergibt sich dann, wenn die Probanden, die eine Teilnahme zugesagt haben, ihre Zusage zurückziehen oder nicht zum vereinbarten Termin erscheinen oder beim Erscheinen mitteilen, dass sie doch nicht begleitet werden wollen. Genauer gesagt: Eine dritte Selektivität ist dann gegeben, wenn sich diese Verweigerer von den übrigen Probanden systematisch unterscheiden. Auch die dritte Selektivität lässt sich nicht vermeiden, aber ebenfalls durch geeignete Maßnahmen wie z.B. Überredungskünste etwas reduzieren.

Die zweite Aufgabe einer künftigen Erforschung der KBM in Sachen externe Validität betrifft die Reaktivität. Das Reagieren auf den Umstand, beim Einkaufen von einem oder von mehreren Forschern begleitet zu werden, kann verschiedene Ursachen oder Auslöser haben. Erstens die Anfrage und die Bitte, an einer Kaufbegleitungsstudie teilzunehmen, zweitens die Vorbefragung, drittens die Ankündigung einer Nachbefragung und viertens die Kaufbegleitung im Laden selbst.

Eine Forschung, die die Auswirkungen solcher Sachverhalte auf das innere und äußere Verhalten beim Einkaufen näher untersucht, kann von unterschiedlichen Annahmen ausgehen, so z.B. von folgenden: Die Zustimmung zur Teilnahme an einer KB-Studie kann bewirken, dass sich der Proband auf den Kauf besser vorbereitet, Kaufpläne besser überlegt, vielleicht sogar ganz unüblich einen Einkaufszettel schreibt, bei dieser Aufzeichnung z.B. tabuisierte Produkte außen 
vor lässt, oder gar mehr Geld mitnimmt als üblich, um nicht als eine ,arme Existenz" angesehen zu werden. Von der Vorbefragung können zusätzliche Effekte ausgehen, so z.B. eine besondere Sensibilisierung für das eigene Verhalten beim Einkaufen und für mögliche Erwartungen der Begleitung an dieses Verhalten. Vielleicht gibt die Begleitperson ganz unbeabsichtigt erste Hinweise darauf, worauf sie besonders achten wird und was sie letztlich herausfinden will. Solche Effekte könnten durch die Ankündigung einer Nachbefragung verstärkt werden, insbesondere das Streben des Probanden, nicht nur durch sein Verhalten beim Einkauf, sondern auch durch seine Auskünfte in der Nachbefragung ein ,gutes Bild“ abzugeben. Denkbar wären aber auch ganz andere Absichten, so z.B. der Wunsch, den Begleiter zu verwirren oder gar in die Irre zu führen. Bezüglich der Kaufbegleitung wäre dann zu bedenken, was allein schon die Beobachtung bewirken kann. Was die Beobachtung bewirkt, dürfte vor allem vom Selbstbild und von der erwünschten Fremdwahrnehmung abhängen. Will ein Verbraucher als ein sparsamer Mensch wahrgenommen werden, dürfte er unter Beobachtung dazu neigen, weniger als sonst einzukaufen. Ganz anders wird sich jener Verbraucher verhalten, der als großzügig, kauffreudig und kaufkräftig wahrgenommen werden möchte. Kommen während des Kaufs ungewohnte Aufgaben auf ihn zu, so z.B. die Aufgabe, all seine Gedanken laut auszusprechen, kann dies nicht nur Hemmungen oder besondere Darstellungsabsichten auslösen, sondern auch kognitive Ressourcen binden und von den üblichen Aufgaben, z.B. von Kaufüberlegungen, abziehen. Wenn ein unübliches Verhalten auch anderen Ladenbesuchern auffallen kann, ist nicht auszuschließen, dass allein daraus Reaktivität erwachsen oder verstärkt werden kann.

Vorliegende Abhandlungen zur KBM sprechen meist nur die Reaktivität während der Kaufbegleitung an, wenn sie auf die Reaktivitätsproblematik überhaupt eingehen. Dabei wird eingeräumt, dass ein Einfluss der Kaufbegleitung auf das Verhalten und Erleben des Ladenbesuchers nicht auszuschließen ist. Solchen Zugeständnissen folgt dann oft der Hinweis, dass sich die begleiteten 
Personen im Laufe der Zeit an die Begleitung gewöhnen und die Reaktivität der Methode deshalb im Laufe der Zeit abnehme (so z.B. Lowrey et al. 2005 S. 179). Auch dieser Sachverhalt sollte künftig gezielt untersucht werden, um herauszufinden, wann welches Ausmaß an Gewöhnung eintritt und welche Reaktivität sich dabei zurückbildet.

In diesem Zusammenhang gilt es im Übrigen zu beachten, was die Erforschung des gemeinsamen Einkaufens bereits an Erkenntnissen zutage gefördert hat. Eine zentrale und wiederholt zutage geförderte Auswirkung einer natürlichen Kaufbegleitung hat ergeben, dass Familienmitglieder den Impulskauf eher hemmen, Freunde und gute Bekannte dagegen eher fördern. Vor diesem Hintergrund lässt sich vermuten, dass von einem unbekannten Begleiter eher kaufanregende als kaufhemmende Wirkungen ausgehen (s. dazu die Studien von Youn \& Faber 2000 S. 181, Mangleburg et al. 2004 S. 110f. sowie Luo 2005 S. 291). Vielleicht verstärken sich die kaufanregenden Effekte sogar, wenn der forschende Begleiter immer stärker als guter Bekannter oder gar als Freund angesehen wird, ein Sachverhalt, den die Protagonisten der KBM meist als ein positives Moment, als einen Zuwachs an Vertrautheit, darstellen.

Neben der Reaktion eines Ladenbesuchers auf den Umstand, dass ihn eine dritte Personen, ein Forscher, begleitet, ist auch die Reaktion auf ganz bestimmte Registriermethoden, z.B. auf die Videographie, zu bedenken. So zeigten sich in einer Studie von Büttner \& Silberer (2007) die erwarteten Reaktivitätseffekte: Die Tatsache, dass die Probanden während des Ladenbesuchs eine Kopfkamera trugen, führte nicht nur zu erhöhter Selbstaufmerksamkeit, sondern auch dazu, dass für die Suchaufgabe im Laden mehr Zeit aufgewandt wurde. Eine künftige Methodikforschung kann die Reaktivitätseffekte bei neuen Forschungsmethoden nicht ausklammern.

\section{Zusammenfassung}

Zur Methode der Kaufbegleitung lässt sich zusammenfassend festhalten, dass es sich um ein bestimmtes Vorgehen und ein leistungs- und entwicklungsfähiges 
Methodenbündel handelt, das auch in Zukunft reichhaltige Einsichten in das Einkaufsverhalten der Konsumenten verspricht. Dies kann nicht hoch genug gewertet werden, da das Einkaufsverhalten darüber entscheidet, welchen Markterfolg die Anbieter - Hersteller und Händler - erzielen und welchen Nutzen die Verbraucher aus dem Konsumgüterangebot ziehen.

Das Entwicklungspotenzial der KBM betrifft sowohl deren ethnographische Ausgestaltung als auch deren Ausgestaltung im Sinne der klassischen, standardisierten und reproduzierbaren Verhaltensforschung. Vielversprechend ist die ethnographische Richtung vor allem im Dienste der Theoriebildung (Entdeckungszusammenhang) und die verhaltensorientierte Richtung im Dienste der Theorieüberprüfung (Begründungszusammenhang). Dies spricht für eine produktive Koexistenz bzw. für eine gezielte Koppelung beider Richtungen. Das Fernziel sollte die „gute Theorie des Einkaufsverhaltens" sein, die der Praxis wertvolle Einsichten und Entscheidungshilfen liefert - nicht nur der Gestaltung und Vermittlung von Angeboten auf der Seite der Hersteller und Händler, sondern auch der Arbeit verbraucherpolitisch tätiger Institutionen.

\section{Literatur}

Agafonof, N. (2006).

Adapting Ethnographic Research Methods to ad hoc Commercial Market Research, in: Qualitative Market Research: An International Journal, Vol. 9 (2006), No 2, pp. 115-125

Alexis, M., Haines, G. H. \& Simon, L. (1968).

Consumer Information Processing: The Case of Women's Clothing, in: King, Robert L. (Ed.). Marketing and the New Science of Planning. Denver, Colorado: American Marketing Association

Barnard, A. (2000).

History and Theory in Anthropology, Cambridge: Cambridge University Press

Belk, R. W. \& Kozinets, R V. (2005)

Videography in Marketing and Consumer Research, in: Qualitative Market Research: An International Journal, Vol. 8 (2005), No 2, pp. 128-141 
Bettmann, J. (1970).

Information Processing Models of Consumer Behavior, in: Journal of Marketing Research, Vol. VII (August 1970), No. 3, pp. 370-376

Bettman, J. \& Zins, M. A. (1977).

Constructive Processes in Consumer Choice, in: Journal of Consumer Research, Vol. 4 (Sept. 1977), pp. 75-85

Büttner, O. B. \& Silberer, G. (2007).

Research Spoilt the Shopping Trip: Comparing the Reactivity of Thinking Aloud and Video-cued Thought Protocols at the Point of Purchase, in: Flexible Marketing in an Unpredictable World, Proceedings of the 36th EMAC Conference, 22-25 May, Reykjavik, Iceland

Büttner, O. B. \& Silberer, G. (2008).

Video-cued Thought Protocols: A Method for Tracing Cognitive Processes at the Point of Purchase, in: Lowrey, T. M. (ed.), Brick \& Mortar Shopping in the 21st Century. New York: Lawrence Erlbaum Associates, pp. 221-241

Eriksen, . H. (2001).

Small Places, Large Issues, London: Pluto Press

Groves, R. M., Cialdini, R. B. \& Couper, M. P. (1992).

Understanding the Decision to Participate in Survey, in: Public Opinion Quarterly, Vol. 56 (1992), No 4, pp. 475-495

Groves, R. M., Singer, E. \& Coming, A. (2000).

Leverage-Salience-Theory of Survey Participation, in: Public Opinion Quarterly, Vol. 64 (2000), No 3, pp. 299-308

Grovers, R. M., Presser, S. \& Dipko, S. (2004).

The Role of Topic Interest in Survey Participation Decisions, in: Public Opinion Quarterly, Vol. 68 (2004), No 1, pp. 2-31

Hammersley, M. \& Atkinson, P. (1983).

Ethnography - Principles and Practice, London: Routledge

Hitzler, R. (2007).

Ethnographie, in: Buber, Renate \& Holzmüller, Hartmut (Hg.). Qualitative Marktforschung, Wiesbaden: Gabler

Iyer, E. S. (1989).

Unplanned Purchasing: Knowledge of Shopping Environment and Time Pressure, in: Journal of Retailing, Vol. 65 (1989), No 1, pp. 40-57 
Jackson, P., Perez del Aguila, R., Clarke, I., Hallsworth, A., de Kervenoael, R. \& Kirkup, M. (2006).

Retail Restructuring and Consumer Choice 2. Understanding Consumer Choice at the Household Level, in: Environment and Planning A, Vol. 38 (2006), No 1, pp. 47-67

Johnson, S. M. \& Bolstad, O. D. (1973).

Methodological Issues in Naturalistic Oberservation, in: Hamerlynck, Leo A., Handy, Lee C. \& Mash, Eric J. (Eds.). Behavior Change: Methodological Concepts and Practice, Paper Presented at the Banff International Conference on Behavior Modification, March 1972, pp. 17-58

King, R. H. (1969).

A Study of the Problem of Building a Model to Simulate the Cognitive Process of a Shopper in a Supermarket, in: Haines, George H. (Ed.). Consumer Behavior: Learning Models of Purchasing, New York: The Free Press, pp. 22-68

Koch, J. (1997).

Marktforschung - Begriffe und Methoden, 2. Aufl., München: Oldenbourg

Kuper, A. (2002).

Anthropology \& Anthropologists, GB: Routledge

Lowrey, T. M., Otnes, C. C, \& Shrum, L. J. (1998).

Consumer Ambivalence: Perspectives Gained from Shopping with Consumers, in: Balderjahn, Ingo, Mennicken, Claudia \& Vernette, Eric (Eds.). New Developments and Approaches in Consumer Behavior Research, Stuttgart: Schäffer-Poeschel, pp. 307-320

Lowrey, T. M. \& Otnes, C. C. (2004).

Consumer Fairy Tales of the Perfect Christmas: Villains and Other Dramatis Personae, in: Otnes, Cele C. \& Lowrey, Tina M. (Eds.). Contemporary Consumption Rituals: A Research Anthology, Mahwah NJ. - London: Erlbaum, pp. 99-122

Lowrey, T. M., Otnes, C. C, \& McGrath, M. A. (2005).

Shopping With Consumers: Reflections and Innovations, in: Qualitative Market Research: An International Journal, Vol. 8 (2005), No 8, pp. 176-188

Luo, X. (2005).

How Does Shopping With Others Influence Impulsive Purchasing? in: Journal of Consumer Psychology, Vol. 15 (2005), No 4, pp. 288-294 
Mangleburg, T. F., Doney, P. M. \& Bristol, T. (2004).

Shopping With Friends and Teens' Susceptibility to Peer Influence, in: Journal of Retailing, Vol. 80 (2004), No 2, pp. 101-116

McGrath, M. A. \& Otnes, C. C. (1995).

Unacquainted Influencers: When Strangers Interact in the Retail Setting, in: Journal of Business Research, Vol. 32 (1995), No 3, pp. 261-272

Miller, D. (1998).

A Theory of Shopping, Ithaca, NY: Cornell University Press

Müller, K. E. (1972, 1980).

Geschichte der antiken Ethnographie und ethnologischen Theoriebildung, Zwei Bände, Wiesbaden: Steiner

Otnes, C. C., Kim, Y. D. \& Lowrey, T. M. (1992).

Ho, Ho, Woe: Christmas Shopping for "Difficult" People, in: Advances in Consumer Research, Vol. 19 (1992), No 1, pp. 482-487

Otnes, C. C. \& Lowrey, T. M. (1993).

Til Dept Do Us Part: The Selection and Meaning of Artifacts in the American Wedding, in: Advances in Consumer Research, Vol. 20 (1993), No 1, pp. 325-329

Otnes, C. C., McGrath, M. A. \& Lowrey, T. M. (1995).

Shopping With Consumers - Usage as Past, Present and Future Research Technique, in: Journal of Retailing and consumer Services, Vol. 2 (1995), No 5, pp. 97-110

Otnes, C. C., Lowrey, T. M. \& Shrum, L. J. (1997).

Toward an Understanding of Consumer Ambivalence, in: Journal of Consumer Research, Vol. 24 (1997), No 6, pp. 80-93

Otnes, C. C. (1998).

"Friends of the Bridge" and Then Some: Role of the Bridal Salon During Wedding Planning, in: Sherry, John F. (Ed.). Service Scapes: The Concept of Place in Contemporary Markets, Lincolnwood, IL: NTC Business Books, pp. 229-257

Otnes, C. C., Lowrey, T. M. \& Kim, Y. C. (2003).

Gift Selection for Easy and Difficult Recipients: A Social Roles Interpretation, in: Journal of Consumer Research, Vol. 20 (Sept. 2003), pp. 229-244 
Park, C., Whan, I., Easwar, S. \& Smith, D. C. (1989).

The Effects of Situational Factors on In-Store Grocery Shopping Behavior: The Role of Store Environment and Time Available for Shopping, in: Journal of Consumer Research, Vol. 15 (1989), No 4, pp. 422-433

Payne, J. W. \& Ragsdale, E. E. K. (1978).

Verbal Protocols and Direct Observation of Supermarket Shopping Behavior: Some Findings and a Discussion of Methods, in: Advances in Consumer Research, Vol. 5 (1979), pp. 571-577

Sherry, J. F. Jr. (1998).

Soul of the Company Store: Nike town Chicago and the Emplaced BrandScape, in: Sherry, J. F. Jr. (Ed.). Service Scapes: The Concept of Place in Contemporary Markets, Lincolnwood, IL: NTC Business Books, pp. 109-146

Silberer, G. (1989).

Die Bedeutung und Messung von Einkaufserlebnissen im Handel, in: V. Trommsdorff (Hg.). Handelsforschung 1989, Jahrbuch der Forschungsstelle für den Handel (FfH), Wiesbaden: Gabler, S. 59-76

Silberer, G. \& Büttner, O. B. (2004).

Video-Cued Thought Protocols as a Method for Assessing Consumer Cognition at the Point of Sale, Beiträge zur Trackingforschung Nr. 7, Göttingen: Georg-AugustUniversität, Institut für Marketing und Handel

Silberer, G. (2005a).

A New Approach to Assessing Cognitive Processes at the Point of Sale - VideoCued Thought Protocols. Beiträge zur Trackingforschung Nr. 10, Göttingen: Georg-August-Universität, Institut für Marketing und Handel

Silberer, G. (2005b).

Die videogestützte Gedankenrekonstruktion kognitiver Prozesse beim Ladenbesuch, in: Marketing ZFP, 27. Jg., Heft 4, S. 263-271

Silberer, G. (2005c).

Das Verhalten am POS - klassische und neuere Möglichkeiten seiner Erfassung. Beiträge zur Trackingforschung Nr. 12, Göttingen: Georg-August-Universität, Institut für Marketing und Handel

Silberer, G. (2006a).

Erkundung des Kundenlaufs und des Zuwendungsverhaltens am Point of Sale. Beiträge zur Tracking-Forschung Nr. 15, Göttingen: Georg-August-Universität, Institut für Marketing und Handel 
Silberer, G. (2006b).

Behavior at the POS - Classical and Newer Methods of Recording It, Paper presented at the 25th Annual Advertising and Consumer Psychology Conference, May 19-21, 2006, Houston

Silberer, G. (2008).

Behavior at the POS - Classical and Newer Methodes of Recording It, in: Tina M. Lowrey (Ed.). Brick \& Mortar Shopping in the 21th Century, New York - London: Lawrence Erlbaum Associates, pp. 257-280

Silberer, G. \& Büttner, O. B. (2008).

Marktforschung am Point of Sale, in: Andreas Herrmann, Christian Homburg \& Martin Klarmann (Hg.). Handbuch Marktforschung, 3. Aufl., Wiesbaden: Gabler, S. $1097-1123$

Titus, P. A. \& Everett, P. B. (1996).

Consumer Wayfinding Tasks, Strategies, and Errors: An Exploratory Field Study, in: Psychology \& Marketing, Vol. 13 (1996), No 3, pp. 265-290

Wells, W. D. \& LoSciuto, L. (1966).

Direct Observation of Purchasing Behavior, in: Journal of Marketing Research, Vol. 3 (1966), No 3, pp. 227-233

Xia, L. (2003).

A Multi-Method Investigation of Consumer Browsing Behaviors and Unintended Information Acquisition, Doctoral Dissertation, Department of Business Administration, University of Illinois at Urbana-Champaign, Urbana, IL.

Youn, S. \& Faber, Ronald J. (2000).

Impulse Buying: It's Relation to Personality Traits and Cues, in: Advances in Consumer Research, Vol. 27 (2000), pp. 179-185 
E

Zur Selektivität der Verhaltensforschung am Point-of-Sale 


\section{Einleitung}

Das Verhalten der Besucher am Point-of-Sale ist in seiner Bedeutung für den Absatzerfolg der Anbieter unbestritten. Dasselbe gilt für die Analyse dieses Verhaltens (vgl. Cobb \& Hoyer 1986, S.385). Deshalb ist es wichtig, die Aussagekraft entsprechender Analysen zu sichern.

Die Aussagekraft der Verhaltensforschung am Point-of-Sale (POS) hängt von mehreren Faktoren ab, vor allem von der internen Validität, aber auch von der externen Validität der Befunde, d.h. von der Reaktivität der Probanden und der Selektivität der Stichproben. Agieren die Probanden reaktiv und sind die Stichproben selektiv, leidet darunter die Generalisierbarkeit der Befunde und damit deren Wert für jene Forscher und Entscheider, die auf entsprechende Erkenntnisse angewiesen sind.

Die bislang durchgeführten Forschungsarbeiten zum Kundenverhalten am POS haben die Reaktivität der Probanden und die Reaktivität der Stichproben vernachlässigt. Und es ist anzunehmen, dass dies nicht nur für wissenschaftliche Untersuchungen zutrifft, sondern auch für die Analysen der Anbieter und die Studien der kommerziellen Marktforschungsinstitute. In diesem Beitrag wollen wir uns deshalb mit der Selektivität der Verhaltensforschung am POS näher beschäftigen. Am Beispiel der wichtigsten Instrumente, der Befragung und Beobachtung, soll uns die Frage interessieren, wann die Verhaltensforschung am POS mit welchen Selektivitätseffekten rechnen muss.

Der Versuch, die Gefahr von Selektivitätseffekten näher zu betrachten und anhand gezielter Studien abzuschätzen, wird sich auf die Verhaltensforschung in Ladengeschäften konzentrieren, zumal noch immer recht viele Käufe im stationären Handel getätigt werden. Angesichts der wachsenden Bedeutung des Online-Vertriebs bzw. des Online-Shopping sei aber festgehalten, dass viele der im Folgenden getroffenen Aussagen - aber nicht alle - auf den Online-Bereich übertragen werden können. 
Nach einer Betrachtung der verschiedenen Formen und Ursachen der Selektivität in der Erforschung des Verhaltens von Ladenbesuchern wird von zwei Studien berichtet, die erste Anhaltspunkte zur Selektivität der Verhaltensforschung am POS liefern. Der Beitrag schließt dann mit konkreten Vorschlägen für künftige Forschungsarbeiten auf diesem Gebiet.

\section{Selektivitätseffekte der Befragung und Beobachtung im Überblick}

Die Selektivität einer Verhaltensstudie kann Unterschiedliches bedeuten: die Auswahl des interessierenden Verhaltens, die Auswahl der agierenden Personen, die Auswahl des Zeitpunktes und anderes mehr. Damit ist die beabsichtige Selektion angesprochen. Die Selektivität, die uns hier interessiert, betrifft jedenfalls die nicht erwünschte, nicht beabsichtigte Selektion. Es geht um die Abweichung der tatsächlich realisierten Stichprobe von der geplanten Stichprobe (vgl. Breakwell 1995, Wilkinson 1995, Bortz \& Döring 1995). Auch wenn diese Selektivität ein „allgemeines Problem der empirischen Sozialforschung“ darstellt (so Winship \& Mare 1992 S.328), soll hier lediglich die Selektivität bei Befragungs- und Beobachtungsstudien vor Ort interessieren.

\section{(1) Zur Selektivität einer Vor-Ort- Befragung}

Ist eine Befragung von Besuchern eines bestimmten Areals, z.B. eines Ladengeschäfts, angestrebt, so kann die realisierte Stichprobe von der geplanten, z.B. repräsentativen Auswahl aus folgenden Gründen abweichen:

- Die Auswahl der Zeiten, in denen die Befragung stattfindet, hat dazu geführt, dass die anvisierte Stichprobe verfehlt wurde, weil zu unterschiedlichen Zeiten unterschiedliche Besucher erreicht wurden.

- Die Auswahl der Standorte, an denen Personen angesprochen wurden, erzeugte Selektivität, weil an den anderen Eingänge oder anderen Orten andere Besucher anzutreffen gewesen wären, aber nicht angesprochen wurden. 
- Die Auswahl der Interviewer, die eingesetzt wurden, erzeugte eine Selektivität, weil diese - bewusst oder unbewusst - bestimmte Besucher überdurchschnittlich oder unterdurchschnittlich oft ansprachen oder durch ihr Aussehen und Verhalten bei bestimmten Besuchern unterschiedliche Ansprecherfolge realisierten.

- Die Gestaltung der Ansprache von Auskunftspersonen und deren Begründung erzeugten Selektivität, weil sie nicht alle Angesprochenen gleichermaßen zum Mitmachen motivieren bzw. überzeugen konnten.

- Der Einsatz von Anreizen wie z. B. Gutscheine und Gewinnchancen haben einen Selektivitätseffekt dadurch verursacht, dass sich die Aussichten auf Erfolg auf die Teilnahmebereitschaft bei einzelnen Personen ganz unterschiedlich auswirkten.

Es liegt in der Hand des Untersuchungsleiters, geeignete Maßnahmen zu ergreifen, die die Selektivität einer Befragung reduzieren. Ganz vermeiden lassen sich solche Effekte allerdings nie (so auch Krosnick 1999 S.539). Unterschiede bezüglich der Erreichbarkeitschancen und der Teilnahmebereitschaften seitens der Zielpersonen lassen sich nicht beseitigen (Theobald 2000 S.123-125). Hervorgehoben sei das spezifische Interesse seitens der Zielpersonen. Die Bereitschaft an einer Studie teilzunehmen steigt mit dem Interesse am Gegenstand der Studie, dem „topic interest“. Dieser Involvement-Effekt kann jedoch an Stärke verlieren, wenn finanzielle Anreize dargeboten werden, so der Befund bei Groves, Presser \& Dipko (2004 S.13). Des weiteren sei auf den Zeitdruck verwiesen, der von den Auskunftspersonen empfunden wird. Auch dieser kann ganz unterschiedlich ausgeprägt und unterschiedlich verteilt sein, was ebenfalls Selektivität erzeugen würde. Wir stimmen Koch (1998) zu, wenn er feststellt, dass sich die Stichprobenverzerrung bei Umfragen nicht einfach durch eine Aufstockung der Stichprobe vermeiden lässt. 


\section{(2) Zur Selektivität einer vor Ort realisierten Beobachtung}

Die Selektivität einer Beobachtung ergibt sich ebenfalls aus den gewählten Beobachtungszeiten, den Beobachtungsstandorten und den beobachtenden Personen. Die Teilnahme an einer Beobachtungsstudie hängt entscheidend davon $\mathrm{ab}$, ob es sich um eine verdeckte oder um eine offene Beobachtung handelt. Bei der offenen Beobachtung ist sich der Beobachtete des Umstandes bewusst, dass er beobachtet werden soll. Die angesprochene Person kann über die Teilnahme frei entscheiden, und daraus erwächst Selektivität. Im Falle einer verdeckten Beobachtung weiß die beobachtete Person nicht, dass sie beobachtet wird. Eine Einwilligung wird nicht erbeten, kann aber aus rechtlichen und ethischen Gründen geboten sein.

Bei der offenen Beobachtung (vgl. Johnson \& Bolstad 1973 S.18f., Wildman \& Erickson 1977 S.265f.) hängt die Selektivität unter anderem davon ab,

- ob die Zielpersonen zu unterschiedlichen Besuchszeiten unterschiedlich bereit sind, sich beobachten zu lassen,

- ob Beobachter bei den zu Beobachtenden mit unterschiedlichem Erfolg eine Zustimmung zur Beobachtung erlangen

- und ob die Begleitung der zu beobachtenden Personen zu unterschiedlichen Zeiten und an unterschiedlichen Orten unterschiedliche Bedenken gegen eine Beobachtung hegt und ggf. für eine Ablehnung der Beobachtung plädiert.

Bei der verdeckten Beobacbtung sind auf den ersten Blick keine Selektivitätseffekte zu erwarten, weil keine Erlaubnis erbeten bzw. erteilt werden muss. Dennoch ist auch hier eine Selektivität denkbar, so z.B. dann,

- wenn die Beobachter selektiv vorgehen und die zu beobachtenden Personen nach bestimmten Kriterien auswählen bzw. ansprechen,

- wenn sich Beobachter in unterschiedlicher Weise verhalten, und zwar dergestalt, dass dies zu selektiven Abbrüchen der Beobachtung führt, 
- oder wenn sich das Bemerken der Beobachtung unterschiedlich verteilt und es ohne Zutun der Beobachter zu „selektiven Abbrüchen“ des Beobachtungsprozesses kommt.

\section{(3) Zur Kontrolle von Selektivitätseffekten}

Geht es darum, das Auftreten von Selektivitätseffekten einer Befragung oder einer Beobachtung zu kontrollieren, so ist vor allem an zwei Maßnahmen zu denken: zum einen an den Vergleich der realisierten Stichprobe mit der angestrebten Stichprobe, z.B. der Vergleich der im Laden befragten Käuferstichprobe mit der Struktur der Bevölkerung der jeweiligen Gemeinde, zum anderen an den Vergleich der bereitwilligen Teilnehmer mit den Verweigerern bzw. Abbrechern (vgl. dazu Cobb \& Hoyer 1986 S.391f.). Der Vergleich der realisierten mit der angestrebten Stichprobe kann daran scheitern, dass eine zuverlässige, aktuelle „Definition der Sollstichprobe“ fehlt. Und beim Vergleich von Teilnehmern und Verweigerern kann das Problem auftauchen, dass sich bei den Verweigerern die erforderlichen Vergleichsmerkmale nicht bestimmen lassen. Im günstigen Falle können bei den Verweigerern einige wenige, schnell erfassbare Merkmale festgehalten werden, so z.B. das Geschlecht der Person und die Anzahl ihrer Begleiter.

\section{Zwei Studien zur Selektivität der Befragung und Beobachtung von Ladenbesuchern}

Die beiden Studien, von denen hier berichtet werden soll, dienten zum einen der Erfassung des Verhaltens am POS, zum anderen sollten sie aber auch erste Einschätzungen möglicher Selektivitätseffekte solcher Untersuchungen liefern, in denen Ladenbesucher zu ihrem Verhalten befragt und in ihrem Verhalten beobachtet werden.

\subsection{Zur Anlage der Studien}

Um Näheres zum Verhalten am POS in Erfahrung zu bringen und erste Anhaltspunkte bezüglich der Selektivität der Befragung und Beobachtung von 
Ladenbesuchern zu gewinnen, wollten wir unterschiedliche Geschäftstypen mit unterschiedlichen Zielgruppen erfassen. Deshalb wurde das Management eines Verbrauchermarktes und eines Fachmarktes angesprochen. Von beiden Unternehmen erhielten wir die Erlaubnis, in einem begrenzten Zeitraum ausgewählte Ladenbesucher zu beobachten und zu befragen. Die Untersuchungen sollten auf eine bestimmte Abteilung begrenzt werden. Sie waren außerdem im Spätherbst durchzuführen, so dass es sich anbot, die zu dieser Zeit in beiden Geschäften eingerichteten Weihnachtsabteilungen auszuwählen und dort das Kundenverhalten näher zu untersuchen. Die beiden Weihnachtsabteilungen waren von vergleichbarer Größe, differierten jedoch in der Angebotsstruktur. Beim Angebot in der Weihnachtsabteilung des Verbrauchermarktes dominierten Süßigkeiten und Weihnachtsartikel, beim Angebot in der Weihnachtsabteilung des Fachmarktes waren Dekorationsmaterial, Weihnachtsschmuck sowie Kerzen und Leuchten vorherrschend.

Die Fokussierung unserer Untersuchung auf ein begrenztes Areal sollte nicht nur aus der Sicht des Managements, sondern auch aus unserer Sicht bewirken, dass die Belastung der teilnehmenden Besucher, aber auch die Gefahr begrenzt bleibt, dass die verdeckte Kundenbeobachtung bemerkt wird. Die räumliche Begrenzung sollte es außerdem erlauben, dass die Beobachter die Abteilungsbesucher recht gut beobachten und außerdem hinreichend genau und schnell registrieren können, wie sich diese vor Ort verhalten. Schließlich sollte die räumliche Begrenzung dazu beitragen, dass sich die Besucher in der Befragung an die ursprünglichen Absichten sowie an das Verhalten vor Ort erinnern können.

In der Verbrauchermarktstudie wurden die vom Beobachter per Zufall auszuwählenden Besucher der Weihnachtsabteilung unauffällig beobachtet, was ohne Ausnahme gelang (vgl. dazu Wells \& LoSciuto 1966), und beim Verlassen der Abteilung mündlich befragt (zur Kombination von Befragung und Beobachtung s. z.B. Granbois 1968, Hoyer 1984, Cobb \& Hoyer 1986). Im Beobachtungsprotokoll waren die Laufwege und die Zuwendungen zum Angebot, nämlich Anschauen, 
Anfassen und Mitnehmen, festzuhalten (vgl. dazu Titus \& Everett 1996, Larson, Bradlow \& Faden 2005). Das anschließende Interview hatte vor allem die Aufgabe, Besuchsmotive, Kaufabsichten, Überlegungen und Urteile bzw. Zufriedenheiten zu erfassen. Als Belohnung für die Teilnahme an der Befragung erhielten die Teilnehmer einen Weihnachtsmann aus Schokolade. Von den 188 beobachteten Besuchern der Weihnachtsabteilung waren 158 bereit, sich befragen zu lassen; 30 lehnten die Befragung ab, wurden aber noch gebeten, den Grund der Ablehnung zu benennen. Wie bei allen Teilnehmern wurden auch bei den Verweigerern die Eintrittszeit, das Geschlecht und die Anzahl der Begleitpersonen festgehalten und eine Beurteilung des Erscheinungsbildes sowie eine grobe Einschätzung des Alters vorgenommen. Dieses Vorgehen erlaubt einen Vergleich der Verweigerer mit den Teilnehmern und liefert somit relativ breit gestreute Hinweise auf die Selektivität einer Befragung von Ladenbesuchern.

In der Fachmarktstudie wurden die per Zufall auszuwählenden Besucher der Weihnachtsabteilung ebenfalls beobachtet und bei Verlassen der Abteilung befragt. Die Gegenstände bzw. Inhalte der Beobachtung und Befragung waren mit denen in der Verbrauchermarktstudie vergleichbar. Allerdings wurde im Fachmarkt etwa die Hälfte der Besucher verdeckt beobachtet, die andere Hälfte dagegen offen, d.h. mit deren Wissen und Zustimmung. Von 114 angesprochenen Besuchern stimmten 82 einer Beobachtung zu, nur 32 lehnten eine Beobachtung ab. Recht erfreulich war auch die Teilnahme an der Befragung. Bei den 82 Besuchern, die offen und mit Erlaubnis beobachtet wurden, konnten alle 82 Personen befragt werden. Von den 100 verdeckt beobachteten Besuchern lehnten nur 9 Personen eine Befragung ab, so dass hier Befragungsergebnisse für 91 Befragte vorliegen. Diese Situation versetzt uns ebenfalls in die Lage, mögliche Selektivitätseffekte einer Befragung und Beobachtung von Ladenbesuchern zu untersuchen.

\subsection{Befunde zur Selektivität der Befragung von Ladenbesuchern}

In der Verbrauchermarktstudie wurden - wie gesagt - insgesamt 188 Besucher in ihrem Lauf- und Zuwendungsverhalten beobachtet, ohne davon zu wissen. Bei der 
anschließenden Bitte um ein Interview stimmten 158 Besucher zu, 30 Besucher lehnten ab. Betrachten wir zunächst die angegebenen Gründe im Falle einer Interviewabsage. Wie die Häufigkeiten in Tabelle 1 zeigen, dominiert die Antwort „keine Zeit“. Einige gaben an, „keine Lust“ zu haben, andere machten deutlich, dass sie eine Befragung grundsätzlich ablehnen.

Tabelle 1: Grïnde der Befragungsverweigerung (Verbrauchermarktstudie)

\begin{tabular}{llll}
\hline keine Zeit & $46 \%(14)$ & & \\
keine Lust & $27 \%(8)$ & lehnen Befragung grundsätzlich ab & $14 \%(4)$ \\
sonstige Gründe & $10 \%(3)$ & keine Angaben & $3 \%(1)$ \\
\hline \hline Zusammen & & $100 \%(30)$ \\
\hline
\end{tabular}

Fallzahlen in Klammern

Da sowohl für die Teilnehmer an der Befragung als auch für die Ablehner der Befragung bereits vor der Bitte um ein Interview reichhaltige Beobachtungsdaten gewonnen werden konnten, kann die Gefahr einer Selektivität der Befragung anhand der Beobachtungsergebnisse abgeschätzt werden. Beobachtet wurden zunächst Personenmerkmale wie das Geschlecht und die Anzahl der Begleitpersonen; die Einschätzung der Altersklasse und die Einstufung des Erscheinungsbildes kommt hinzu. Darüber hinaus wurden per Beobachtung Verhaltensmerkmale festgehalten, vor allem das Lauf-, Zuwendungs- und Kaufverhalten, aber auch die Eintrittszeit, die Aufenthaltsdauer und die Nutzung eines Einkaufswagens.

Vergleich der Personenmerkmale: Für die Personen, die an der Befragung teilnahmen, und die Personen, die eine Befragung ablehnten, wurden folgende Merkmale registriert: Geschlecht, Anzahl der Begleitpersonen, (geschätzte) Altersklasse, (taxiertes) Äußeres, Eintrittszeit, Benutzung eines Einkaufswagens und Fülle des Einkaufswagens. Die in Tabelle 2 festgehaltenen Resultate zeigen keine signifikanten Unterschiede bezüglich dieser Merkmale und somit auch keine entsprechende Selektivität einer Kundenbefragung am POS. 
Tabelle 2: Merkmale der Befragungsteilnehmer/ablehner im Verbrauchermarkt

\begin{tabular}{lccc}
\hline & $\begin{array}{c}\text { Teilnehmer an d. Befr. } \\
(\mathrm{n}=158)\end{array}$ & $\begin{array}{c}\text { Ablehner der Befr. } \\
(\mathrm{n}=30)\end{array}$ & $\begin{array}{c}\text { Signifikanz der } \\
\text { Differenz }\end{array}$ \\
\hline Geschlecht (Frauenanteil) & $80,89 \%$ & $86,36 \%$ & n. s. \\
Anzahl Begleitpersonen & $1,46(0,65)$ & $1,38(0,68)$ & n. s. \\
(geschätzte) Altersklasse $^{1)}$ & $2,06(0,69)$ & $2,24(0,69)$ & n. s. \\
Aussehen (Outfit) ${ }^{2)}$ & $2,15(0,83)$ & $2,17(0,85)$ & n. s. \\
Eintrittszeit (Std.: Min.) & $14: 24(2: 41)$ & $14: 04(2: 29)$ & n. s. \\
Benutzung Einkaufswagen & $76,44 \%$ & $65,52 \%$ & n. s. \\
Fülle Einkaufswagen ${ }^{3)}$ & $1,56(0,77)$ & $1,25(0,58)$ & n. s. \\
\hline
\end{tabular}

${ }^{1)}$ Skala: $1=$ bis $24 \mathrm{~J} . ; 2=25-45 \mathrm{~J} . ; 3=46 \mathrm{~J}$. und älter

2) Skala: 1 = einfach; $2=$ weder noch, $3=$ gut

3) Skala: 1 = leer; 2 = wenig; $3=$ viel

Tests: beim Geschlecht, bei der Altergruppe und der Benutzung eines Einkaufswagens der $\chi^{2}$ -

Test nach Pearson; ansonsten: t-Test, zweiseitig; n. s. = auf dem 5\%-Niveau nicht signifikant;

Standardabweichung in Klammern.

Vergleich der Verhaltensmerkmale: Da bei den Befragungsteilnehmern und den Befragungsablehnern das Lauf- und Zuwendungsverhalten, die Produktmitnahme eingeschlossen, beobachtet wurden, lässt sich diese Frage nach Gruppenunterschieden im POS-Verhalten beantworten. Tabelle 3 zeigt die Ergebnisse des Verhaltensvergleichs, auch des Vergleichs in Bezug auf die Dauer des Aufenthalts in der Abteilung.

Das Ergebnis des Verhaltensvergleichs zeigt, dass sich Teilnehmer und Ablehner in ihrem Verhalten am POS bis auf eine Ausnahme, die vom Beobachter eingeschätzte Eile, nicht unterscheiden. Die Befragungsablehner hatten es offenbar eiliger als die Befragungsteilnehmer. Unterschiede bezüglich der Besuchsdauer und bezüglich der registrierten Merkmale des beobachteten Lauf- und Zuwendungsverhaltens existieren nicht. Das gilt auch für die sog. Laufmuster. Um Laufmuster zu erkennen, haben wir die Flächenkontaktsequenzen näher betrachtet und miteinander verglichen. Die Distanzmatrix deutete bereits auf einige homogene Sequenzcluster hin. Eine sequenzbasierte Clusteranalyse bzw. eine Clusterung der Flächenkontaktsequenzen ergab drei unterschiedliche Cluster und somit drei 
Laufmuster, auf deren Darstellung hier allerdings verzichtet werden soll. Das erste Laufmuster umfasste 27 Personen, das zweite Laufmuster 88 Personen und das dritte Laufmuster 73 Personen. Wie sich die Laufmuster auf die Interviewteilnehmer und Interviewablehner verteilen, zeigt Tabelle 4. Das Resultat macht deutlich, dass auch keine Unterschiede bezüglich der Laufmuster existieren.

Tabelle 3: Verhalten der Befragungsteilnehmer/ablehner im Verbrauchermarkt

\begin{tabular}{lccc}
\hline & $\begin{array}{c}\text { Teilnehmer } \\
(\mathrm{n}=158)\end{array}$ & $\begin{array}{c}\text { Ablehner } \\
(\mathrm{n}=30)\end{array}$ & $\begin{array}{c}\text { Signifikanz der } \\
\text { Differenz }\end{array}$ \\
\hline \hline Aufenthaltsdauer (Min.: Sec.) & $4: 42(3: 21)$ & $3: 58(2: 18)$ & n. s. \\
\hline Anzahl betretener Flächen & $11,77(5,79)$ & $10,90(3,96)$ & n. s. \\
Laufweg in m & $71,89(35,83)$ & $66,85(25,96)$ & n. s. \\
Laufgeschwindigkeit (m/Min.) & $20,35(14,55)$ & $19,94(8,98)$ & n. s. \\
\hline Eile $(0=$ nein, 1 = weder noch; 2 ja) & $0,39(0,70)$ & $1,45(0,83)$ & $* * *$ \\
\hline Anzahl der Zuwendungen & $4,69(2,47)$ & $5,13(2,90)$ & n. s. \\
$\begin{array}{l}\text { Zuwendungen im einzelmen: } \\
\text { Produktgruppe betrachtet }\end{array}$ & $0,18(0,61)$ & $0,20(0,55)$ & n. s. \\
bestimmtes Produkt angeschaut & $1,38(1,37)$ & $1,57(1,68)$ & n. s. \\
Produkt herausgenommen & $1,56(1,92)$ & $1,23(1,74)$ & n. s. \\
Produkt mitgenommen & $1,69(1,76)$ & $1,33(1,35)$ & n. s. \\
\hline
\end{tabular}

Test: t-Test, zweiseitig; $* * *=$ signifikant auf dem 0,1 \%-Niveau, n. s. $=$ auf dem 5\%-Niveau nicht signifikant; Standardabweichung in Klammern

Tabelle 4: Laufmuster der Interviewteilnehmer/ablehner im Verbrauchermarkt

\begin{tabular}{lcc}
\hline & Teilnehmer $(\mathrm{n}=158)$ & Ablehner $(\mathrm{n}=30)$ \\
\hline \hline Sequenzcluster 1 $(\mathrm{n}=27)$ & $14,6 \%(23)$ & $13,3 \%(4)$ \\
Sequenzcluster 2 $(\mathrm{n}=88)$ & $43,7 \%(69)$ & $63,3 \%(19)$ \\
Sequenzcluster 3 $(\mathrm{n}=73)$ & $41,8 \%(66)$ & $23,3 \%(7)$ \\
\hline zusammen & $100 \%(158)$ & $100 \%(30)$
\end{tabular}

Test: $\chi^{2}$-Test; $\chi^{2}=4,32(\mathrm{df}=2)$; zweiseitig; Unterschiede auf dem $5 \%$-Niveau nicht signifikant. Fallzahlen in Klammern 


\subsection{Befunde zur Selektivität der Beobachtung von Ladenbesuchern}

In der Fachmarktstudie wurden ein Teil der Ladenbesucher verdeckt beobachtet, der andere Teil sollte offen beobachtet werden und war daher anzusprechen und um Erlaubnis zu bitten. Eine erste Selektivität, durch die Ansprache seitens der Beobachter bedingt, kann darin bestehen, dass sich die Gruppe der zum Zwecke einer offenen Beobachtung angesprochenen Personen von der Gruppe der verdeckt Beobachteten unterscheidet. Es könnte vorgekommen sein, dass die Beobachter bewusst oder unbewusst selektiert haben. Es könnte aber auch sein, dass sich bestimmte Ladenbesucher leichter ansprechen ließen als andere.

Wie sich aus Tabelle 5 ergibt, unterscheidet sich die zwecks offener Beobachtung angesprochenen Gruppe (A-Gruppe) von der Gruppe der verdeckt Beobachteten (VB-Gruppe) weder in Bezug auf das Geschlecht, noch in Bezug auf die Anzahl der Begleitpersonen, wohl aber in Bezug auf die Eintrittszeit: die um Zustimmung zu einer Beobachtung gebetenen bzw. angesprochenen Personen waren etwas früher eingetreten als die verdeckt beobachteten Ladenbesucher. Die Anfrage und das Bitten um Erlaubnis zur Beobachtung hat offenbar Zeit gekostet, vor allem das Auffinden der angestrebten Anzahl an Personen, die einer Beobachtung zustimmen.

Tabelle 5: Merkmale der der zwecks offener Beobachtung angesprochenen Personen und der verdecket beobachteten Personen (Fachmark.tstudie)

\begin{tabular}{lccc}
\hline & $\begin{array}{c}\text { A-Gruppe } \\
(\mathrm{n}=114)\end{array}$ & $\begin{array}{c}\text { VB-Gruppe } \\
(\mathrm{n}=100)\end{array}$ & $\begin{array}{c}\text { Signifikanz der } \\
\text { Differenz }\end{array}$ \\
\hline Geschlecht (Frauenanteil) & $78,95 \%$ & $79,00 \%$ & n. s. \\
Anzahl der Begleitpersonen & $0,53(0,68)$ & $0,46(0,67)$ & n. s. \\
Eintrittszeit (Std.: Min.) & $14: 29(2: 26)$ & $13: 45(2: 07)$ & $* *$
\end{tabular}

Tests: beim Geschlecht $\chi^{2}$-Test; beim Rest: t-Test, zweiseitig; ** = signifikant auf dem 1\%Niveau, n. s. $=$ auf dem 5\%-Niveau nicht signifikant. Standardabweichung in Klammern; AGruppe $=$ zwecks offener Beobachtung angesprochene Personen; VP-Gruppe $=$ verdeckt beobachtete Personen 
Über den Vergleich der Teilnehmer und der Verweigerer in Sachen offene Beobachtung lässt sich eine weitere, žweite Selektivität prüfen, eine Selektivität, die sich vor allem auf die Teilnahmebereitschaft bei einer offenen Beobachtung bezieht. Von den um Zustimmung zur Beobachtung gebetenen Ladenbesuchern haben sich nicht alle beobachten lassen: 82 stimmten der Beobachtung zu, 32 lehnten eine Beobachtung ab. Da für die Ablehner weder Beobachtungsergebnisse noch Ergebnisse aus der Befragung beim Verlassen der Abteilung vorliegen, muss sich dieser Vergleich auf schnell registrierbare und tatsächlich registrierte Merkmale und auf die angegebenen Verweigerungsgründe beschränken. Das Ergebnis eines derartigen Vergleichs findet sich in Tabelle 6. Beobachtungsteilnehmer unterscheiden sich von den Ablehnern bezüglich der Anzahl der Begleitpersonen: sie sind häufiger in Begleitung als Beobachtungsablehner. Sie haben die Abteilung auch etwas früher als die Ablehner betreten. Vielleicht hat das spätere Eintreffen damit zu tun, dass weniger Zeit und Geduld zur Verfügung stand. Unterschiede in Bezug auf das Geschlecht konnten nicht festgestellt werden. Aufschlussreich sind deshalb die angegebenen Verweigerungsgründe. Tabelle 7 zeigt eine klare Dominanz der Antwort „keine Zeit“.

Tabelle 6: Teilnebmer an und Ablebner der offenen Beobachtung (Fachmarktstudie)

\begin{tabular}{lccc}
\hline & $\begin{array}{c}\text { Teilnehmer an der OB } \\
(\mathrm{n}=82)\end{array}$ & $\begin{array}{c}\text { Ablehner der OB } \\
(\mathrm{n}=32)\end{array}$ & $\begin{array}{c}\text { Signifikanz der } \\
\text { Differenz }\end{array}$ \\
\hline \hline Geschlecht (Frauenanteil) & $78,05 \%$ & $81,25 \%$ & n. s. \\
Anzahl Begleitpersonen & $0,60(0,72)$ & $0,34(0,55)$ & $*$ \\
Eintrittszeit (Std.: Min.) & $14: 52(2: 24)$ & $13,30(2: 16)$ & $* *$ \\
\hline
\end{tabular}

Tests: beim Geschlecht $\chi^{2}$-Test nach Pearson, $\chi^{2}(\mathrm{df}=1)=0,142$, Rest: t-Test, zweiseitig; */** = signifikant auf dem 5/1\%-Niveau, n. s. $=$ auf dem $5 \%$-Niveau nicht signifikant. Standardabweichung in Klammern. $\mathrm{OB}=$ offene Beobachtung.

Aus dem Vergleich der tatsächlich verdeckt und offen beobachteten Personen lassen sich Hinweise auf eine weitere, dritte Selektivität herauslesen (s. Tabelle 8). Es war offenbar leichter und/oder verlockender, jüngere Personen für die offene 
Beobachtung zu gewinnen. Es fiel wohl auch weniger schwer, solche Ladenbesucher zu gewinnen, die sich in der Abteilung nur umschauen bzw. bummeln wollten und denen vielleicht auch etwas mehr Zeit zur Verfügung stand (zum Shopping ohne Kaufabsicht s. Bloch \& Richins 1983). Dass Personen, die die Abteilung vorher bereits schon einmal oder mehrfach besucht hatten, unter den verdeckt Beobachteten etwas stärker vertreten waren, könnte sich aus dem Umstand ergeben, dass die Wiederholer unter den Besuchern der Abteilung relativ häufig vertreten waren - ein Umstand, der allerdings nicht kontrolliert wurde. Schließlich zeigt sich, dass sich verdeckt und offen beobachtete Besucher auch in Bezug auf die Eintrittszeit unterscheiden.

Tabelle 7: Angegebene Gründe für die Ablehnung der Beobachtung (Fachmarktstudie)

\begin{tabular}{cccc}
\hline keine Zeit & $75,0 \%(24)$ & Begleitung wartet & $9,4 \%(3)$ \\
keine Lust & sonstige Gründe & $6,2 \%(2)$ \\
\hline \hline zusammen & $9,4 \%(3)$ & $100 \%(32)$ \\
\hline
\end{tabular}

Fallzahlen in Klammern

Tabelle 8: Merkmale verdeckt und offen beobachteter Ladenbesucher (Fachmarktstudie)

\begin{tabular}{lccc}
\hline & $\begin{array}{c}\text { OB-Gruppe } \\
(\mathrm{n}=82)\end{array}$ & $\begin{array}{c}\text { VB-Gruppe } \\
(\mathrm{n}=100)\end{array}$ & $\begin{array}{c}\text { Signifikanz der } \\
\text { Differenz }\end{array}$ \\
\hline \hline Geschlecht (Frauenanteil) & $78,05 \%$ & $79,00 \%$ & n. s. \\
Anzahl Begleitpersonen & $0,60(0,72)$ & $0,47(0,69)$ & n. s. \\
(erfragtes) Alter (bei VB: $\mathrm{n}=91)$ & $42,54(15,54)$ & $47,21(14,58)$ & $*$ \\
Eintrittszeit (Std.: Min.) & $14: 52(2: 24)$ & $13: 41(2: 07)$ & $* * *$ \\
Besuchshäufigkeit (bei VB: $\mathrm{n}=91)$ & $1,84(1,13)$ & $2,21(1,11)$ & $*$ \\
Besuchsgründe: & & & $* * *$ \\
- Schauen/Bummeln & $35,37 \%$ & $12,09 \%$ & n. s. \\
- Inspiration/Information & $7,32 \%$ & $3,30 \%$ & n. s. \\
- Produktkauf & $34,15 \%$ & $42,86 \%$ & \\
\hline
\end{tabular}

Tests: Geschlecht und Besuchsgründe: $\chi^{2}$-Test nach Pearson, Rest: t-Test, zweiseitig; */*** = signifikant auf dem 5,0/0,1\%-Niveau, n. s. $=$ auf dem 5\%-Niveau nicht signifikant, beim Alter wurde wegen der Verletzung der Normalverteilungs-Bedingung ein Mann Whitney U-Test durchgeführt. Standardabweichung in Klammern; $\mathrm{OB}=$ offene Beobachtung, $\mathrm{VB}=$ verdeckte Beobachtung. 


\subsection{Zusammenfassung der wichtigsten Ergebnisse}

Die Befunde unserer Studien bezüglich der Selektivität einer Befragung und Beobachtung von Ladenbesuchern lassen insgesamt nur wenige bzw. recht begrenzte Selektivitätseffekte erkennen. Teilnehmer und Verweigerer bei der Besucherbefragung im Verbrauchermarkt glichen sich bezüglich des Geschlechts, der Begleitpersonenanzahl, der geschätzten Altersklasse, des taxierten Aussehens, der Eintrittszeit, der Benutzung und Fülle des Einkaufswagens und der Laufmuster. Allerdings hatten es die Ablehner etwas eiliger als die Teilnehmer. Bei der Beobachtungsstudie im Fachmarkt hatten die Angesprochenen und die verdeckt Beobachteten denselben Frauen- bzw. Männeranteil und die gleiche Anzahl an Begleitpersonen.

Die Teilnehmer an der offenen Beobachtung ähnelten auch denen, die diese Beobachtung nicht wünschten, was das Geschlecht angeht, betraten die Abteilung allerdings etwas früher und hatten außerdem mehr Begleitung. Schließlich ähnelten sich auch die offen und die verdeckt Beobachteten, und zwar in Bezug auf den Geschlechteranteil, die Begleitpersonenanzahl, den Besuchsgrund „Information“ und den Besuchsgrund „Kaufen“. Allerdings waren die offen Beobachteten jünger, kamen etwas später, berichteten eine geringere Besuchshäufigkeit in der Vergangenheit und wollten sich viel häufiger nur etwas umschauen. Bezogen auf die Begründung jener Besucher, die sich nicht befragen bzw. beobachten lassen wollten, ist festzuhalten, dass jeweils an erster Stelle der Mangel an Zeit genannt worden war.

Angesichts unserer Befunde sollte auch bedacht werden, was aus den beiden Studien nicht herausgelesen werden kann. Dazu zählen Selektivitätseffekte bei Befragungen und Beobachtungen, die sich nicht nur für das Verhalten in bestimmten Abteilungen interessieren, sondern für das gesamte Besucherverbalten in großen Geschäften oder ganzen Einkaufszentren. Dasselbe gilt für Studien, in denen die Befragung mit der Beobachtung kombiniert wird, so z.B. beim Einsatz der Kaufbegleitungsmethode. Nicht untersucht wurde auch die mögliche Entfaltung 
längerfristiger Selektivitätseffekte, die darin bestehen können, dass die Bereitschaft, an einer späteren Studie teilzunehmen, positiv oder negativ beeinflusst wird.

\section{Folgerungen für die Verhaltensforschung am Point-of-Sale}

Insgesamt gesehen sind die Ergebnisse für stichprobenartige Befragungs- und Beobachtungsstudien am POS recht ermutigend, deuten sie doch auf sehr begrenzte Selektivitätseffekte hin. Sie sprechen eher für als gegen die Berechtigung, Ergebnisse von Besucherbefragungen und -beobachtungen zu generalisieren, auch wenn dabei eine differenzierte Betrachtung einzelner Sachverhalte durchaus geboten ist.

Da Selektivitätseffekte jedoch nicht auszuschließen sind, liegen folgende Empfehlungen nahe: Erstens die Kontrolle solcher Effekte, zweitens deren Einschränkung und drittens deren Beachtung bei der Auswertung und Interpretation von Studienergebnissen.

\section{(1) Zur Kontrolle möglicher Selektivitätseffekte}

Selektivitätseffekte lassen sich am besten - wie oben bereits aufgezeigt - durch geeignete Studiendesigns kontrollieren. Wichtig ist dabei die Analyse der Verweigerer und der Abbrecher in ihren personenbezogenen Merkmalen, aber auch - soweit dies möglich ist - in ihren Verhaltensweisen vor Ort.

\section{(2) Zur Einschränkung möglicher Selektivitätseffekte}

Selektivität lässt sich dadurch begrenzen, dass Beobachter und Interviewer in Bezug auf ihr Mitwirken bei der Auswahl und Ansprache von Ladenbesuchern sensibilisiert und kontrolliert werden. Werden die Teilnahme- bzw. Ablehnungsentscheidungen seitens der Ladenbesucher und deren Ursachen analysiert, so z.B. deren Reaktionen auf die Ansprache, auf Bitten und auf Versprechungen, besteht die Chance, entsprechende Maßnahmen zu verbessern bzw. so zu gestalten, dass die Teilnahmebereitschaft steigt. 


\section{(3) Zur Beachtung der Selektivität in der Auswertung und Interpretation der}

\section{Studienergebnisse}

Weicht eine realisierte Stichprobe von der Soll-Stichprobe nachweislich ab, können die Probanden so gewichtet werden, dass die gewichtete Stichprobe der SollStichprobe entspricht. Dieses Verfahren setzt jedoch voraus, dass genügend Fälle vorhanden sind und dass die unterrepräsentierten Fälle für ihre Gruppe halbwegs repräsentativ sind. Außerdem ist zu beachten, dass die Inferenzstatistik reale Stichproben voraussetzt und keine hochgerechneten. Werden die Defizite einer Stichprobe schnell erkannt, sollte deshalb versucht werden, noch in der Phase der Datenerhebung die Stichprobenziehung so anzupassen, dass die Zahl jener Probanden steigt, die ansonsten unterrepräsentiert wären. Sind solche Bemühungen nicht möglich oder erfolglos, muss die faktische Selektivität offen gelegt und bei der Ergebnisinterpretation beachtet werden. Dies bedeutet, dass für unterrepräsentierte Personengruppen gar keine Aussagen oder nur Aussagen unter Vorbehalt getroffen werden.

Je stärker künftige Verhaltensstudien am POS auf Selektivitätseffekte achten und damit auf die Grenzen einer Verallgemeinerung von Befunden, desto nützlicher werden die Folgerungen sein, die aus den Studienergebnissen gezogen werden. Dies gilt für betriebliche und kommerzielle Analysen im Dienste des Anbieter-Marketing ebenso wie für Untersuchungen jener Forscher, die eine gute Theorie des Käuferverhaltens entwickeln bzw. testen wollen.

\section{Literatur}

Bloch, P. H. \& Richins, M. L. (1983).

Shopping Without Purchase: An Investigation of Consumer Browsing Behavior, in: Advances in Consumer Research, Vol. 10 (1983), pp. 389-393

Bortz, J. \& Döring, N. (1995).

Forschungsmethoden und Evaluation für Sozialwissenschaftler, 2. Aufl., Berlin: Springer 
Breakwell, G. M. (1995).

Interviewing. in: G. M. Breakwell, S. Hammond, \& C. Fife-Shaw (Eds.). Research Methods in Psychology, London: SAGE, pp. 230-242

Cobb, C. J. \& Hoyer, W. D. (1986).

Planned Versus Impulse Purchase Behavior, in: Journal of Retailing, Vol. 60 (1986), No 4, pp. 384-409

Granbois, D. H. (1968).

Improving Study of Customer In-store Behavior, in: Journal of Marketing, Vol. 32 (1968), No 4, pp. 28-33

Groves, R. M., Presser, S. \& Dipko, S. (2004).

The Role of Topic Interest in Survey Participation Decisions, in: Public Opinion Quarterly, Vol. 68 (2004), No 1, pp. 2-31

Hoyer, W. D. (1984).

An Examination of Consumer Decision-Making for a Common Repeat Purchase Product, in: Journal of Consumer Research, Vol. 11 (1984), No 3, pp. 822-829

Johnson, S. M. \& Bolstad, O. D. (1973).

Methodological Issues in Naturalistic Observation: Some Problems and Solutions for Field Research, in: L. A. Hamerlynck, L. C. Handy, \& E. J. Mash (Eds.). Behavior Change: Methodology Concepts and Practise, Champaign, IL: Research Press, pp. 7-67

Koch, A. (1998).

Wenn "Mehr" nicht gleichbedeutend mit "Besser" ist: Ausschöpfungsquoten und Stichprobenverzerrung in allgemeinen Bevölkerungsumfragen. ZUMA-Nachrichten, 42. Jg. (1998), S. 66-90

Krosnick, J. A. (1999).

Survey Research, in: Annual Review of Psychology, Vol. 50 (1999), pp. 537-567

Larson, J. S., Bradlow, E. T., \& Faden, P. S. (2005).

An Exploratory Look at Supermarket Shopping Paths, in: International Journal of Research in Marketing, Vol. 22 (2005), No 4, pp. 395-414

Theobald, A. (2000).

Das World Wide Web als Befragungsinstrument. Wiesbaden: Gabler

Titus, P. A., \& Everett, P. B. (1996).

Consumer Wayfinding Tasks, Strategies and Errors: An Exploratory Field Study, in: Psychology \& Marketing, Vol. 13 (1996), No 3, pp. 265-290 
Wells, W. D. \& LoSciuto, L. A. (1966).

Direct Observation of Purchasing Behavior, in: Journal of Marketing Research, Vol. 3 (1966 Aug), pp. 227-233

Wildman, B. G. \& Erickson, M. T. (1977).

Methodological Problems in Behavioral Observation, in: J. D. Cone \& R. P. Hawkins (Eds.). Behavioral Assessment: New Directions in Clinical Psychology, New York: Brunner/Mazel, pp. 255-273

Wilkinson, J. (1995).

Direct Observation, in: G. M. Breakwell, S. Hammond, \& C. Fife-Schaw (Eds.). Research Methods in Psychology, London: SAGE, pp. 213-229

Winship, C. \& Mare, R. D. (1992).

Models for Sample Selection Bias, in: Annual Review of Sociology, Vol. 18 (1992), pp. 

Zur Reaktivität der Verhaltensforschung am Point-of-Sale 


\section{Einleitung}

Das Verhalten der Besucher am Point-of-Sale ist in seiner Bedeutung für den Absatzerfolg der Anbieter unbestritten. Dasselbe gilt für die Analyse dieses Verhaltens (vgl. Cobb \& Hoyer 1986, S.385). Deshalb ist es wichtig, die Aussagekraft entsprechender Analysen zu sichern.

Die Aussagekraft der Verhaltensforschung am Point-of-Sale (POS) hängt von mehreren Faktoren ab, vor allem von der internen Validität, aber auch von der externen Validität der Befunde, d.h. von der Reaktivität der Probanden und der Selektivität der Stichproben. Verhalten sich die Probanden reaktiv und sind die Stichproben selektiv, leidet darunter die Generalisierbarkeit der Befunde und damit deren Wert für jene Forscher und Entscheider, die auf entsprechende Erkenntnisse angewiesen sind.

Die bislang durchgeführten Forschungsarbeiten zum Kundenverhalten am POS haben die Reaktivität der Probanden und die Reaktivität der Stichproben vernachlässigt. Und es ist anzunehmen, dass dies nicht nur für wissenschaftliche Untersuchungen zutrifft, sondern auch für die Analysen der Anbieter und die Studien der kommerziellen Marktforschungsinstitute. In diesem Beitrag wollen wir uns deshalb mit der Reaktivität der Verhaltensforschung am POS näher beschäftigen. Am Beispiel der wichtigsten Instrumente, der Beobachtung und Befragung, aber auch der Denke-Laut-Methode, der Videographie und ausgewählter Registriermethoden, soll uns die Frage interessieren, wann die Verhaltensforschung am POS mit welchen Reaktivitätseffekten rechnen muss.

Der Versuch, die Gefahr von Reaktivitätseffekten näher zu betrachten und anhand gezielter Studien abzuschätzen, wird sich auf die Verhaltensforschung in Ladengeschäften konzentrieren, zumal noch immer recht viele Käufe im stationären Handel getätigt werden. Angesichts der wachsenden Bedeutung des Online-Vertriebs bzw. des Online-Shopping sei aber festgehalten, dass viele der im Folgenden getroffenen Aussagen - aber nicht alle - auf den Online-Bereich übertragen werden können. 
Nach einer Betrachtung der verschiedenen Formen und Ursachen der Reaktivität in der Erforschung des Verhaltens von Ladenbesuchern wird von drei Studien berichtet, die erste Anhaltspunkte zur Reaktivität der Befragung und Beobachtung von Ladenbesuchern liefern. Der Beitrag schließt mit konkreten Vorschlägen für künftige Forschung auf diesem Gebiet.

\section{Reaktivitätseffekte in der Verhaltensforschung}

Reaktivität bedeutet, dass Probanden in ihrem inneren bzw. verdeckten und/oder in ihrem offenen Verhalten auf Messungen und deren Ankündigung reagieren. Das dann erfasste Verhalten ist also ein anderes als jenes Verhalten, das ohne Messung bzw. deren Ankündigung zu erwarten gewesen wäre.

Der Komplex „Reaktivitätseffekte der Verhaltensforschung“ kann hier nicht erschöpfend behandelt werden - zu zahlreich sind die möglichen Forschungsinstrumente und die Möglichkeiten, diese kombiniert einzusetzen, und zu zahlreich die möglichen Reaktionen der Probanden sowie die entsprechenden Wirkungsmechanismen. Im Folgenden soll es genügen, wenn einige wichtige Forschungsmethoden und einige wenige Wirkungsmechanismen herausgegriffen werden.

Zur Erinnerung einige Forschungsmethoden, die bei einer Erfassung inneren und äußeren Verhaltens in Frage kommen, und die an anderer Stelle näher dargestellt worden sind: (1) die verdeckte und die offene Beobachtung, bei der ein Beobachter sich passiv, aber auch aktiv verhalten kann, (2) die Videographie bzw. die Aufnahmen mit Raumkameras, die Aufnahmen eines mitlaufenden Kameramannes, die Augenkamera und die Blickfeldkamera, (3) die diversen Befragungsmethoden, so z.B. die gestützte oder ungestützte Nachherbefragung und die Unterbrechermethode, (4) die Denke-Laut-Methode und (5) die gestützte Rekonstruktion des inneren und des äußeren Verhaltens, z.B. die videogestützte Gedankenrekonstruktion.

Der Einsatz solcher Instrumente kann verschiedene Reaktionen der Probanden hervorrufen (s. dazu im Einzelnen Bettman \& Zins 1977 S.75f., Hoc \& Leplat 1983, Russo et al. 1989 S.760, Biggs et al. 1993 S. 191 und van den Haak et al. 
2003 sowie die bei Ericsson \& Simon 1993 S.83 - 107 und bei Büttner 2008 S.7177 referierten Studien). Die verschiedenen Wirkungsmechanismen sollen hier nur grob skizziert werden. Es handelt sich im Einzelnen um folgende Effekte und Mechanismen (ebd): (1) die Beeinflussung der Wahrnehmung, vor allem deren Sensibilisierung und deren Ausrichtung (Selektivität) für die Umwelt und für die eigene Person, so z.B. die Steigerung der Selbstaufmerksamkeit, (2) die Ablenkung von ursprünglich gehegten Absichten oder sich selbst gesetzten Aufgaben und damit einhergehend die Gefahr, diese Absichten zu vernachlässigen und die Aufgaben schlecht oder gar nicht zu erfüllen, (3) der erhöhte Zeitaufwand bei der Erledigung der jeweiligen Aufgaben, (4) die Veränderung der Motivation und die des Anspruchniveaus, in dem ein Proband z.B. als besonders rationaler Käufer oder als ein kaufkräftiger Verbraucher gesehen werden möchte, wobei es vor allem auf die bewussten und unbewussten Signale ankommt, die ein Forscher und seine Helfer aussenden, und (5) eine Beeinträchtigung der Befindlichkeit, so z.B. die Einbuße beim Einkaufserleben, wenn die Gedanken permanent artikuliert werden müssen.

\section{(1) Zur Reaktivität einer Beobachtung}

Reaktivitätseffekte sind am ehesten bei einer offenen Beobachtung zu erwarten. Der Proband weiß, dass er beobachtet wird, und kann den Beobachter ggf. auch gut wahrnehmen. Es liegt nahe, dass der Beobachtete seine Selbstaufmerksamkeit steigert und sich darüber Gedanken macht, was der Beobachter über sein Verhalten denken könnte. In diesem Zusammenhang ist deshalb auch oft von einem verstärkten Streben nach Verhaltenskontrolle die Rede (s. z.B. Johnson \& Bolstad 1973, Kent \& Foster 1977, Wildman \& Erickson 1977, Haynes \& Horn 1982). Mag eine natürliche alltägliche Begleitung durch Freunde, Verwandte oder Bekannte erwünscht sein und als angenehm empfunden werden, ist das Gegenteil bei einer von Dritten erbetenen Beobachtung nicht auszuschließen. Dann kann es auch vorkommen, dass der zu beobachtende Vorgang verkürzt bzw. abgebrochen wird. 
Selbst die Zielsetzungen der Probanden können sich beobachtungsbedingt ändern (vgl. Chandon, Morwitz \& Reinartz 2004, Dholakia \& Morwitz 2002).

Eine Beobachtung kann aber auch verdeckt, d.h. ohne Wissen des Beobachteten erfolgen (Adler \& Adler 1994, S.382). Es liegt die Vermutung nahe, dass es zu keiner Reaktivität kommt, weil der Beobachtete nicht weiß, dass er beobachtet wird. Dennoch kann auch hier eine Reaktivität ausgelöst werden, nämlich dann, wenn die verdeckt beobachtete Person bemerkt oder auch nur vermutet, dass sie beobachtet wird. Allein die Anwesenheit einer dritten Person kann schon das Verhalten einer Person beeinflussen. Der anwesenden dritten Person muss keine Beobachterrolle zugeschrieben oder zugedacht worden sein.

Beim Einsatz der Beobachtungsmethode ist im übrigen zu bedenken, dass beobachtete Personen, die von der Beobachtung wissen oder diese vermuten, den Beobachter ebenfalls beobachten, damit auch dessen Verhalten beeinflussen und damit eine soziale Interaktion auslösen können (Smith, McPhail \& Pickens 1975 S. 538-540). Es handelt sich dann um eine ,interaktiven Reaktivität“, deren Effekte besonders stark ausgeprägt sein können.

\section{(2) Zur Reaktivität in der Videographie}

Unbemerkte Videoaufnahmen sind in Sachen Reaktivität ähnlich zu beurteilen wie die verdeckte Beobachtung, und bemerkte Aufnahmen ähnlich wie die offene Beobachtung. Unbemerkte Kameras wären z.B. Raumkameras, ggf. auch die an bestimmten Regalen montierten Miniatur-Kameras. Augenkameras und Blickfeldkameras, die am Kopf des Probanden befestigt werden, sind anders zu beurteilen. Sie erzeugen ungewohnte Empfindungen und bei Dritten ggf. den sog. Gaffereffekt. Die Gefahr ist groß, dass in solchen Fällen die Selbstaufmerksamkeit in besonderem Maße auf die Wahrnehmung der eigenen visuellen Zuwendung gelenkt wird und das Verhalten sowie die Handlungssituation alles andere als unbeschwert empfunden werden. Diese Gefahr ist dort besonders groß, wo dem Probanden „,vorgeschrieben“ wird, in welchem Abstand oder von welchem Punkt 
aus er ein Regal betrachten soll bzw. betrachten darf. Beim mitlaufenden Kameramann liegt die Parallele zur teilnehmenden Beobachtung besonders nahe. Hier kommen dann auch die Person und das Verhalten des Beobachters ins Spiel, so dass auch bestimmte Erwartungen signalisiert und verhaltenswirksam werden können.

\section{(3) Zur Reaktivität verschiedener Registrierungsmethoden}

Bei der Erfassung des Kundenlaufs im Laden können die RFID-Methode oder die mobilfunkbasierte Ortung zum Einsatz kommen. Werden dabei lediglich Einkaufwagen oder Einkaufskörbe geortet, dürfte sich die Reaktivität der Besucher in Grenzen halten, auch dann, wenn diese von der Registrierung wissen. Stärkere Reaktionen sind bei einer Personenortung zu erwarten, wenn diese - wie erforderlich - bekannt gemacht wurde. Eine Reaktion des Besuchers könnte darin bestehen, dass er Verkaufszonen mit tabuisierten Produkten meidet und solche Produkte somit auch dann nicht kauft, wenn das Interesse durchaus besteht. Vielleicht findet auch eine Sensibilisierung für das eigene Laufverhalten statt, auch die Bereitschaft, noch nie betretene Zonen einmal aufzusuchen.

\section{(4) Zur Reaktivität verschiedener Befragungsmethoden}

Werden z.B. Ladenbesucher vor, während oder nach Beendigung eines Ladenbesuchs befragt, und ist diese Befragung nicht angekündigt und auch nicht aufgrund anderer Hinweise, z.B. aufgrund von Hinweisen seitens schon befragter Kunden, zu erwarten, ist mit Reaktivitätseffekten nicht zu rechnen. Ist eine solche Befragung erfolgt, dann kann allerdings nicht ausgeschlossen werden, dass das Verhalten danach beeinflusst wird. Es könnte z.B. sein, dass sich ein befragter Besucher an alte Kaufwünsche erinnert und diese vor Ort unverzüglich nachholt.

Wird einem Geschäftsbesucher eine Befragung angekündigt, kann dies dazu führen, dass der Befragte sein Verhalten bewusster wahrnimmt, gezielter steuert und vielleicht Dinge unbeachtet lässt, die er ansonsten anschauen, prüfen oder gar 
kaufen würde. Vielleicht wählt er einen Ausgang, an dem er dem Interview ausweichen kann. Die Reaktion eines Ladenbesuchers auf die Ankündigung einer Befragung hängt jedenfalls davon $a b$, welches Befragungsziel und welches Befragungsthema angekündigt werden, welche Befragungsmethode zum Einsatz kommen soll (mündliche oder schriftlich Befragung), welche Befragungsabsichten und welche Befragungsinhalte der Besucher vermutet bzw. erwartet und ob der Proband davon ausgehen muss, dass der Interviewer die Möglichkeit hat, die von ihm gekauften Produkte zu sehen und damit die Möglichkeit hat, gezielt nach den Kaufgründen zu fragen.

Das Wissen um eine Befragung kann das nachfolgende Verhalten auf verschiedene Art und Weise beeinflussen. Webb et al. (1966, S.13-16) unterscheiden zwei Effekte: den Versuchspersoneneffekt und Rolleneffekt. Der Versuchspersoneneffekt bestehe darin, dass sich der Proband bewusst ist oder wird, Gegenstand einer Untersuchung zu sein. Dies kann eine erhöhte Selbstaufmerksamkeit und den Versuch stimulieren, eine gute Versuchsperson zu sein. Der Rolleneffekt bestehe darin, dass die Person untersuchungsbedingt (!) eine bestimmte Rolle auswählt und ausführt, z.B. sich vornimmt, den braven Käufer und nicht den bösen Dieb zu spielen. Auch wenn Webb et al. (ebd) davon ausgehen, dass es sich hier um zwei Effekte handelt, so stellt der Rolleneffekt doch nichts anderes als eine Ausprägung des Versuchspersoneneffekts dar.

Der Versuchspersoneneffekt besteht im Kern darin, dass die betreffende Person ihr Verhalten stärker als sonst kontrolliert, d.h. sich das Verhalten deutlicher vor Augen hält und bewusst steuert (vgl. Johnson \& Bolstad 1973, Kent \& Foster 1977, Wildman \& Erickson 1977, Haynes \& Horn 1982). In welche Richtung das Verhalten dabei gesteuert wird, hängt von der sozialen Erwünschtheit bestimmter Verhaltensweisen und somit von der „social desirability“ ab (s. Paulhus 1991). Bezogen auf eine konkrete Situation sind dann auch „demand characteristics“ der Situation von Bedeutung (s. dazu Orne 1962), die Erwartungen 
des Forschers und seiner Helfer mit inbegriffen (zur sog. „experimenter expectancy“s. z.B. Rosenthal 1966).

\section{(5) Zur Reaktivität der Denke-Laut-Methode}

Die Denke-Laut-Methode muß als die klassische Methode der Erfassung kognitiver Prozesse damit rechnen, dass das zu messende Verhalten aufgrund der Messung zu beeinflusst wird. Auch wenn die Auffassungen über die Reaktivität der DLM auseinander gehen, so besteht doch Einigkeit darüber, dass die Artikulationsaufgabe das Denken selbst beeinflusst. Die Artikulationsaufgabe bindet kognitive Ressourcen, kann die Leistung bei der zu erfüllenden Aufgabe beeinträchtigen, auch die Qualität zu treffender Entscheidungen, verlangsamt oder verzögert die Bearbeitung von Aufgaben und lenkt die Aufmerksamkeit auf Denkinhalte, die ansonsten gar keine nennenswerte Beachtung finden würden. Das Ungewöhnliche an der Erhebungssituation und die Belastung per Artikulation der Gedanken lassen schließlich auch erwarten, dass das interessierende Geschehen an Attraktivität verliert, dass die „Freude an der Sache“ beeinträchtigt wird.

Müssen die Gedanken in Anwesenheit eines Forschers artikuliert werden, ist mit normativen Einflüssen eher zu rechnen als dort, wo lediglich ein Tonband oder ein Diktaphon zum Einsatz kommt. Spricht der Proband in ein Handy und sitzt der Forscher am anderen Ende der Leitung, sind personale Einflüsse ebenfalls nicht auszuschließen. Neben der Präsenz des Forschers kann auch die Präsenz Dritter einen Einfluss nehmen und z.B. dazu beitragen, dass die Situation als unnatürlich empfunden wird und dass bestimmte Sachverhalte in einem sozialen Umfeld nur ungern laut geäußert werden.

\section{(6) Zur Reaktivität der gestützten Rekonstruktion des Verhaltens}

Die Rekonstruktion des zu untersuchenden Verhaltens findet vor allem deswegen erst nach dem interessierenden Verhalten statt, weil keine Reaktivität erzeugt werden soll. Dies gilt für die gestützte Rekonstruktion von offenen Verhaltens- 
weisen ebenso wie für die Rekonstruktion von Gedanken. Mit Reaktivitätseffekten muss allerdings dann gerechnet werden, wenn die Rekonstruktion angekündigt wurde oder wenn sie aus anderen Gründen heraus antizipiert werden kann oder wenn allein die Erstellung der Erinnerungshilfe schon zu Reaktionen führen kann. Letzteres trifft z.B. dann zu, wenn ein Video, das das Verhalten zeigt, helfen soll, bestimmte Verhaltensweisen wie z. B. die schwer einsehbare visuelle Zuwendung oder die jeweils abgelaufenen kognitiven Prozesse zu rekonstruieren. Ansonsten sei hier auf jene Aussagen verwiesen, die oben $\mathrm{zu}$ möglichen Reaktionen eines Probanden auf die Kaufbegleitung, die sich Skizzen macht, auf den mitlaufenden Kameramann, auf die Blickaufzeichnung und auf die Aufzeichnung des Blickfeldes bereits gemacht worden sind.

Wird eine Erinnerungsstütze präsentiert und soll der Proband auf die Erinnerungshilfe in der Weise reagieren, dass er sich an sein Verhalten und an die jeweiligen Verhaltenssituationen bestmöglich erinnert, wäre dies keine Reaktivität im oben definierten Sinne. Bewirkt die Erinnerungshilfe jedoch etwas ganz anders und trägt sie z.B. dazu bei, dass der Proband in seiner Absicht bestärkt wird, bestimmte Dinge nicht preiszugeben, hätte dies eine Einschränkung der Validität der Messung zur Folge.

\section{(7) Zusammenfassung}

Nachdem einige Reaktivitätseffekte in der Verhaltensforschung nach Messmethoden differenziert angesprochen wurden, bleibt zweierlei festzuhalten: Zum einen die Vielfalt möglicher Reaktivitätseffekte und ihrer Bedingungsfaktoren, zum anderen die Tatsache, dass sich die Reaktivität beim kombinierten Einsatz verschiedener Methoden noch potenziert. Eine Möglichkeit der Methodenkombination wurde an anderer Stelle behandelt, nämlich die Kaufbegleitungsmethode. Dort finden sich auch Hinweise zu möglichen Reaktionen jener Personen, die bei ihrem Einkauf von einem Forscher begleitet werden. 


\section{Studien zur Reaktion von Ladenbesuchern auf die Analyse ihres Verhaltens}

Studien zur Reaktivität in der Verhaltensforschung sind eher selten. Dies gilt in ganz besonderem Maße für Studien, die sich auf die Reaktion von Ladenbesuchern auf die Erfassung ihres Verhaltens beziehen. In der Absicht, diese Forschungslücke zu verringern und Näheres zur Reaktivität in der Verhaltensforschung am POS in Erfahrung zu bringen, wurden in letzter Zeit doch einige Feldexperimente am Institut für Marketing und Handel der Universität Göttingen durchgeführt.

\subsection{Eine Studie zur Reaktion von Ladenbesuchern auf eine offene}

\section{Beobachtung}

\section{(1) Fragestellung und Anlage der Studie}

Die zentrale Frage, deren Beantwortung diese Studie erlauben soll, lautet: Wie reagieren Ladenbesucher auf eine offene Beobachtung, d.h. auf eine Beobachtung, der sie zugestimmt haben und die sie auch wahrnehmen können?

Um erste Einsichten in die Reaktivität einer offenen Beobachtung von Ladenbesuchern zu gewinnen, wurde das Management eines Fachmarktes angesprochen. Dieses erteilte uns die Erlaubnis, Ladenbesucher in einem begrenzten Zeitraum und einer ausgewählten Abteilung zu beobachten. Die sollte im Spätherbst durchgeführt werden, so dass es sich anbot, die zu dieser Zeit eingerichtete Weihnachtsabteilung auszuwählen. Im Angebot der Weihnachtsabteilung waren unter anderem Dekorationsmaterial und Weihnachtsschmuck, festliches Verpackungsmaterial, Tischkerzen und weihnachtliche oder winterliche Beleuchtungen für die Fenster und für den Garten vertreten.

Die per Zufall auszuwählenden Besucher der Weihnachtsabteilung wurden zur Hälfte verdeckt beobachtet, die andere Hälfte dagegen offen, d. h. mit deren Wissen und Zustimmung. Alle beobachteten Personen wurden beim Verlassen der Abteilung befragt. Von den im Rahmen der offenen Beobachtung angesprochenen Besuchern, es waren insgesamt 114 Personen, stimmten 82 einer Beobachtung zu, 
32 lehnten eine Beobachtung ab. Bei den 82 Besuchern, die offen beobachtet wurden, konnten auch alle 82 Personen befragt werden. Von den 100 verdeckt beobachteten Besuchern lehnten 9 Personen eine Befragung ab, so dass auch für 91 verdeckt beobachtete Personen sowohl Beobachtungs- als auch Befragungsergebnisse vorliegen. Diese Situation versetzte uns in die Lage, mögliche Reaktivitätseffekte einer offenen Beobachtung sowohl in Bezug auf das offene Verhalten als auch in Bezug auf das verdeckte Verhalten, soweit es Gegenstand der Befragung war, zu untersuchen.

\section{(2) Auswertung und Darstellung der Ergebnisse}

Um Anhaltspunkte für eine Reaktivität der offenen Beobachtung von Ladenbesuchern zu gewinnen, erfolgt ein Vergleich des offenen und verdeckten Verhaltens der offen Beobachteten mit dem Verhalten der verdeckt Beobachten. Dabei ist allerdings zu bedenken, dass die Ergebnisse eines derartigen Gruppenvergleichs nicht vorschnell als Reaktivitätseffekte interpretiert werden dürfen. Es ist ja nicht auszuschließen, dass beide Gruppen sich schon von vornherein unterscheiden, so dass die (Verhaltens-)Unterschiede nicht ohne weiteres der Beobachtungsreaktivität alleine zugeschrieben werden dürfen.

Die Ergebnisse unserer Fachmarktstudie zeigen, dass sich das Verhalten der offen beobachteten Ladenbesucher vom Verhalten der verdeckt beobachteten nur zum Teil unterscheidet. Die in Tabelle 1 dargestellten Ergebnisse des Gruppenvergleichs lassen erkennen, dass sich die Gruppen sowohl in der Besuchsdauer als auch in ihrem Laufverhalten gleichen. Dies gilt auch für das Zuwendungsverhalten - die Produktmitnahme ausgenommen. Unter den offen beobachteten Besuchern nahmen jedoch mehr Personen Produkte mit als unter den verdeckt beobachteten. Auch nahmen die offen beobachteten Besucher mebr Produkte mit als die verdeckt beobachteten. Deutlich ist auch der Unterschied bei den ungeplanten Käufen: Der Anteil der Besucher, die ungeplante Käufe getätigt 
haben, liegt bei den offen Beobachteten deutlich höher als bei den verdeckt Beobachteten (vgl. dazu Prasad 1975, Cobb \& Hoyer 1986, Iyer 1989).

Um Unterschiede im Laufmuster überprüfen zu können, wurden LaufflächenKontakt-Sequenzen per Clusteranalyse auf Laufmuster hin untersucht (zur Segmentierung von POS-Besuchern s. Lesser \& Hughes 1986, Ruiz et al. 2004). Dabei fanden sich zwei unterschiedliche Cluster und damit zwei grundlegende Laufmuster: Das eine zeigt einen kreisförmigen Lauf dem Uhrzeigersinn entgegen, das andere die Nutzung des Hauptganges in der Mitte der Abteilung sowohl beim Hineingehen als auch bei Herausgehen. Wie die Befunde in Tabelle 2 zeigen, lassen die offen und die verdeckt Beobachteten auch keine unterschiedlichen Laufmuster erkennen.

Tabelle 1: Das Besuchs-, Lauf-, Zuwendungs- und Kaufverhalten offen und verdeckt beobachteter Ladenbesucher

\begin{tabular}{|c|c|c|c|}
\hline & $\begin{array}{c}\text { OB-Gruppe } \\
(\mathrm{n}=82)\end{array}$ & $\begin{array}{l}\text { VB-Gruppe } \\
(\mathrm{n}=100)\end{array}$ & $\begin{array}{l}\text { Signifikanz } \\
\text { der Differenz }\end{array}$ \\
\hline Besuchsdauer (Min.: Sec.) & $4: 10(3: 46)$ & $3: 39(1: 59)$ & n. s. \\
\hline Anzahl betretener Flächen & $15,55(6,05)$ & $15,65(5,59)$ & n. s. \\
\hline Anzahl Zuwendungen & $9,29(7,08)$ & $8,22(4,51)$ & n. s. \\
\hline \multicolumn{4}{|l|}{ Zuwendungen im einzelnen: } \\
\hline Produktgruppe betrachtet & $2,79(2,59)$ & $2,48(1,98)$ & n. s. \\
\hline bestimmtes Produkt angeschaut & $2,52(2,61)$ & $2,57(2,36)$ & n. s. \\
\hline Produkt herausgenommen & $2,96(3,25)$ & $2,80(2,62)$ & n. s. \\
\hline Produkt mitgenommen & $1,01(2,62)$ & $0,37(0,73)$ & * \\
\hline Anzahl mitgenommener Produkte & $1,11(2,69)$ & $0,32(0,63)$ & $* *$ \\
\hline Anteil Impulskäufer (bei VB: $\mathrm{n}=91$ ) & $21,69 \%$ & $5,50 \%$ & $* *$ \\
\hline
\end{tabular}

Tests: beim Anteil Impulskäufer $\chi^{2}$-Test $\mathrm{n}$. Pearson, $\chi^{2}(\mathrm{df}=1)=0,384$; Rest: $\mathrm{t}-$ Test, zweiseitig; * $/ * *$ $=$ signifikant auf dem 5,0/1,0\%-Niveau, n. s. = auf dem $5 \%$-Niveau nicht signifikant, Standardabweichung in Klammern, $\mathrm{OB}=$ offene Beobachtung, $\mathrm{VB}=$ verdeckte Beobachtung 
Tabelle 2: Laufmuster offen und verdeckt beobachteter Ladenbesucher

\begin{tabular}{lcc}
\hline & OB-Gruppe ( $\mathrm{n}=82)$ & VB-Gruppe $(\mathrm{n}=100)$ \\
\hline \hline Laufcluster 1 & $50,0 \%(41)$ & $49,0 \%(49)$ \\
Laufcluster 2 & $50,0 \%(41)$ & $51,0 \%(51)$ \\
\hline Zusammen & $100 \%(82)$ & $100 \%(100)$
\end{tabular}

Test: $\chi^{2}$-Test; $\chi^{2}(\mathrm{df}=1)=0,018$; auf dem 5\%-Niveau nicht signifikant; Fallzahlen in Klammern, $\mathrm{OB}=$ offene Beobachtung, $\mathrm{VB}=$ verdeckte Beobachtung

Ein weiterer Gruppenvergleich bezieht sich auf die Beurteilung des POS, die aufgrund der (Nachher-)Befragung der beobachteten Ladenbesucher erfasst werden konnte. Von besonderem Interesse war hier die Beurteilung der Abteilung, des Besuches und des Einkaufs. Die Ergebnisse dieser Urteilsabfrage finden sich in Tabelle 3. Auch hier zeigen sich keine signifikanten Gruppenunterschiede.

Die festgestellten Unterschiede zwischen dem offen und dem verdeckt beobachteten Verhalten, nämlich der Produktmitnahme und der Impulskäufe, könnten sich nicht nur aus der Beobachtung ergeben, sondern auch aus den vorab existierenden Gruppenunterschieden - das waren die Unterschiede bezüglich des Alters, der Besuchshäufigkeit, des Besuchsgrundes „Schauen/Bummeln“ und der Eintrittszeit. Deshalb war zu prüfen, ob Zusammenhänge zwischen diesen vier Variablen und den festgestellten Unterschieden im POS-Verhalten existieren. Im Rahmen einer Korrelationsanalyse zeigten sich jedoch keine (auf dem 5\%-Niveau) signifikanten Zusammenhänge zwischen dem Alter, der Besuchshäufigkeit, dem Besuchsgrund Schauen/Bummeln und der Eintrittszeit einerseits und der Anzahl mitgenommener Produkte sowie dem Anteil der Impulskäufer andererseits. Deshalb darf davon ausgegangen werden, dass die bei verdeckt und offen beobachteten Ladenbesuchern festgestellten Verhaltensunterschiede der Beobachtung zuzuordnen und damit als Reaktivitätseffekte zu interpretieren sind. 
Tabelle 3: POS-Beurteilung durch offen und verdeckt beobachtete Ladenbesucher

\begin{tabular}{|c|c|c|c|}
\hline & $\begin{array}{l}\text { OB-Gruppe }(\mathrm{n}= \\
82)\end{array}$ & $\begin{array}{l}\text { VB-Gruppe }(\mathrm{n}= \\
\text { 91) }\end{array}$ & $\begin{array}{l}\text { Signifikanz } \\
\text { der Differenz }\end{array}$ \\
\hline 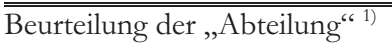 & $3,78(0,80)$ & $3,70(0,88)$ & n. s. \\
\hline Beurteilung des „Besuchs“ ${ }^{\text {2) }}$ & $3,95(0,75)$ & $3,91(0,89)$ & n. s. \\
\hline Beurteilung des „Kaufs“ 2) & $4,13(1,01)$ & $3,82(0,59)$ & n. s. \\
\hline Beurteilung der Preise ${ }^{3)}$ & $2,88(0,73)$ & $2,85(0,87)$ & n. s. \\
\hline $\begin{array}{l}{ }^{1)} \text { Skala: } 1=\text { sehr schlecht } \ldots \ldots \\
{ }^{2)} \text { Skala: } 1=\text { sehr unangenehm . } \\
\text { 3) Skala: } 1=\text { sehr teuer } . \ldots \ldots \ldots . .\end{array}$ & $\begin{array}{l}.5=\text { sehr gut } \\
5=\text { sehr angenehr } \\
. .5=\text { sehr preiswer }\end{array}$ & & \\
\hline
\end{tabular}

Test: t-Test, zweiseitig; n. s. = auf dem 5\%-Niveau nicht signifikant; Standardabweichung in Klammern; $\mathrm{OB}=$ offene Beobachtung, $\mathrm{VB}=$ verdeckte Beobachtung

Aufgrund der Anlage der Studie waren Erkenntnisse bezüglich der Reaktivität einer offenen Beobachtung zu gewinnen, nicht dagegen Erkenntnisse bezüglich der Reaktivität einer verdeckten Beobachtung, welche sich daraus ergeben könnte, dass Ladenbesucher auch auf die Anwesenheit einer Person in gewisser Nähe reagieren. Eine Feststellung der Geschäftsführung könnte mit dieser Reaktivität zu tun haben: Sie berichtete, dass die Diebstahlrate in der Zeit, in der die Studie durchgeführt wurde, um mehr als $50 \%$ sank. Vermutlich lässt sich dieser Effekt nicht allein auf die offene Beobachtung freiwilliger Pobanden zurückführen, auch nicht allein auf die Reaktion jener Ladenbesucher, die eine solche Beobachtung ablehnten, sondern wohl auch auf die Reaktion der verdeckt beobachteten Besucher - vielleicht auch auf die Reaktion jener Besucher, die sich in der Abteilung aufhielten, als die Beobachtung anderer Besucher im Gange war, aber gar nicht angesprochen waren.

\section{(3) Zusammenfassung und Interpretation der Ergebnisse}

Unsere Befunde zeigen insgesamt gesehen, dass die offene Beobachtung von Ladenbesuchern bzw. deren Ankündigung zu kaufbezogenen (Reaktivitäts-) Effekten führen können. Offenbar haben sich die (offen) beobachteten Besucher stärker als andere bemüßigt oder gar verpflichtet gefühlt, etwas zu mitzunehmen bzw. etwas zu kaufen. Vielleicht wollten die offen beobachteten Ladenbesucher 
ihren Besuch gegenüber der beobachtenden Person irgendwie rechtfertigen und sind deswegen viel stärker und/oder viel häufiger in die Käuferrolle geschlüpft, als sie dies - unbeobachtet - getan hätten. Wenn Studien zur Kaufbegleitung feststellen konnten, dass Familienmitglieder als Kaufbegleitung ungeplante Käufe eher hemmen, Freunde und Bekannte dagegen eher stimulieren, so weisen unsere Befunde zum Einfluss neutraler Beobachter bzw. neutraler Begleiter in dieselbe Richtung.

Eine Reaktivität bezüglich der Besuchsdauer, der Anzahl besuchter Flächen und der Laufmuster war ebenso wenig festzustellen wie eine Reaktivität bei der Zuwendung zum Angebot - bei der Häufigkeit einer Produktbetrachtung und der Anzahl jener Fälle, in denen Produkte in die Hand genommen wurden. Die offene Beobachtung erzeugte auch keine Unterschiede in der Beurteilung der Abteilung, des eigenen Besuchs der Abteilung, der getätigten Käufe und der wahrgenommenen Preislage. Die Reaktivität einer offenen Beobachtung scheint sich demnach auf eine stärkere Übernahme der „Käuferrolle“ zu beschränken.

\subsection{Eine Studie zur Reaktion von Ladenbesuchern auf eine Befragung und Beobachtung}

Für die Anbieter spielen ungeplante Käufe, aber auch die Realisierung geplanter Käufe eine zentrale Rolle. Sollen derartige Sachverhalte erfasst werden, liegt es nahe die Kaufpläne vor dem Ladenbesuch zu erfragen und die Gründe für die ungeplanten Käufe sowie die Gründe für die Nichtrealisierung von Kaufplänen nach dem Ladenbesuch $\mathrm{zu}$ erfragen. Soll auch noch das Lauf- und Zuwendungsverhalten erfasst werden, z.B. um zu analysieren, wie Kontakte mit dem Angebot zu ungeplanten Käufen führen, ist die Kombination von Beobachtung und Befragung unumgänglich. In Bezug auf die Reaktivität der Besucher ist daher auch zu fragen, wie die Ladenbesucher auf ein Befragungs- und Beobachtungs-Mix reagieren. 


\section{(1) Fragestellung und Anlage der Studie}

Bei der Anlage der am Institut für Marketing und Handel der Universität Göttingen durchgeführten Studie wurde zunächst davon ausgegangen, dass allein schon die Befragung von Ladenbesuchern zu den Kaufplänen Reaktivitätseffekte erzeugen kann. Um diese bestimmen zu können, wurde beschlossen, einen Teil der Besucher vorab nach den Plänen zu befragen, dem anderen Teil der Besucher jedoch keine derartige Frage zu stellen. In unserem Experimentaldesign erscheint somit die „Vorab-Abfrage der Kaufpläne" als Faktor I.

Gleichzeitig sollte in Erfahrung gebracht werden, welche Reaktivität von der Bitte um Zustimmung zu einer Beobachtung ausgelöst wird. Deshalb fungiert die Bitte um eine Zustimmung zur Beobachtung in unserem Experimentaldesign als Faktor II. Für den Fall einer entsprechenden Zustimmung sollte der jeweilige Ladenbesucher auch davon ausgehen können, dass er tatsächlich beobachtet wird. Von Interesse war des Weiteren die Reaktivität, die von der Ankündigung einer Nachber-Befragung ausgelöst wird. Diese Ankündigung stellt im Experimentaldesign den Faktor III dar.

Das Design unseres Feldexperiments, das sich am besten anhand einer Abbildung (s. Abb. 1) darstellen lässt, unterscheidet somit acht Experimentalbedingungen. Diese Untersuchungsanlage erlaubt es auch, das Zusammenwirken des Faktors I „Abfrage der Kaufpläne“ mit den Faktoren II + III „Bitte um Erlaubnis zur Befragung und/oder Beobachtung" zu untersuchen.

Als abhängige Variable „Reaktivität“ interessierten mehrere Sachverhalte. Als mögliche Ausprägungen einer „Reaktivität“ wurden vor allem das Laufverhalten, das Zuwendungsverhalten (inkl. Produktmitnahme) und kognitive Prozesse angesehen. Deshalb wurde entschieden, dass alle Probanden während des Abteilungsbesuchs beobachtet und nach dem Abteilungsbesuch befragt werden. 
Abbildung 1: Das Design des Feldexperiments

\begin{tabular}{lcccc}
\hline & $\begin{array}{c}\text { weder Befragung noch } \\
\text { Beobachtung erbeten }\end{array}$ & $\begin{array}{c}\text { Beobachtung } \\
\text { erbeten }\end{array}$ & $\begin{array}{c}\text { Befragung } \\
\text { erbeten }\end{array}$ & $\begin{array}{c}\text { Befragung \& } \\
\text { Beobachtung erbeten }\end{array}$ \\
\hline Keine & $(1)$ & $(3)$ & $(5)$ & $(7)$ \\
Kaufplanabfrage & & & & $(8)$ \\
Kaufplanabfrage & $(2)$ & $(4)$ & $(6)$ & ( \\
\hline
\end{tabular}

Die Realisierung unseres Feldexperiments brachte eine Reihe von Hausforderungen mit sich. Zunächst waren möglichst viele Teilnehmer zu gewinnen; die Vorgabe lag bei 30 Probanden pro Experimentalbedingung. Außerdem waren die Verweigerer festzuhalten und einige ihrer Merkmale, um Selektivitätseffekte kontrollieren zu können. Die Probanden waren per Zufall den 8 Experimentalbedingungen zuzuweisen. Und um keine systematische Fehler zu produzieren, sollten die Bobachter und Interviewer systematisch variiert zum Einsatz kommen. Besonders wichtig war es auch, dass die verdeckte Beobachtung von den entsprechenden Probanden nicht bemerkt wird, was bis auf einen Fall gelang, der dann von der Auswertung ausgeschlossen wurde. Für die Beobachtung war ein Ladengrundriss mit dem Eintrag von Lauf- und Präsentationsflächen so zu erstellen, dass er sich sowohl für die Einträge des Lauf- und Zuwendungsverhaltens eignet als auch für die Codierung und Dateneingabe. Messinstrumente, die bei allen Probanden zum Einsatz kamen, waren der Erhebungsbogen bzw. der Ladengrundriss für die Beobachtung und der Fragebogen für die Nachbefragung. Messinstrumente, die den Vorgaben für die einzelnen Experimentalbedingungen entsprechend nur bei bestimmten Probanden zum Einsatz kamen, waren der Bogen mit den Bitten um Zustimmung zu einer Beobachtung und/oder Nachbefragung, der Bogen mit den Fragen für die Erfassung von Kaufplänen und der Bogen mit Fragen an die Ablehner. Der kurze Fragebogen für die Ablehner enthielt auch die Auflistung jener Merkmale, die für diesen Personenkreis zu registrieren waren.

Eine mehrtägige Vorstudie sollte die Handhabung und Verständlichkeit der verschiedenen Messinstrumente sichern. Zum anderen war die Realisierbarkeit der 
Ablauforganisation $\mathrm{zu}$ überprüfen und das Vorgehen vor Ort einzuüben. Außerdem ging es darum, die Mitarbeiter des Geschäfts mit dem Geschehen vertraut zu machen.

\section{(2) Auswertung und Ergebnisse der Studie}

Für die Bestimmung der Reaktivität im Lauf- und Zuwendungsverhalten (Kaufverhalten inklusive) wurden die beobachteten Verhaltensakte ausgezählt und bezüglich der Sequenzen auch häufige Substrings in ihrer Häufigkeit bestimmt. Auf diese Weise entstanden weitere metrische Variablen, die bei den Varianzanalysen ebenfalls untersucht werden sollten.

Die Varianzanalysen erfolgten, um bei ausgewählten abhängigen Variablen aus den Bereichen Lauf-, Zuwendungs- und kognitives Verhalten die Haupteffekte und die Interaktionseffekte der folgenden drei Faktoren zu ermitteln: für den Faktor F 1 „Vorabbefragung der Kaufpläne (Kaufplanabfrage)“, für den Faktor F 2 „erlaubte offene Beobachtung (Beobachtung erbeten)“ und für den Faktor F3 ,erlaubte und angekündigte Nachherbefragung (Befragung erbeten)“. Folgende abhängige Variablen erhielten dabei besonderes Interesse: (1) die Verweildauer, (2) die Anzahl der Laufflächenkontakte, (3) die Anzahl der Zuwendungen „Anschauen“, (4) die Anzahl der Zuwendungen „Anfassen“, (4) die Anzahl der Zuwendungen „Produktmitnahme“, (5) die Anzahl der Kontakte mit dem Personal, (6) die Anzahl „ungeplanter Käufe“, (7) die Anzahl der Kognitionen in Sachen „Ziele und Intentionen“, (8) die Anzahl der Kognitionen in Sachen „Alternativenbewertung“ und (9) die Anzahl aller (berichteten) Kognitionen.

\section{Zur Reaktion auf die Abfrage der Kaufpläne}

Die Varianzanalysen zeigen, dass die Abfrage der Kaufpläne (F1) weder auf das Laufverhalten noch auf das Zuwendungsverhalten signifikante Haupteffekte ausübt (s. unten Tab. 4, 6, 8 \& 10). Es zeigten sich auch keine Interaktionseffekte F1*F2 bzw. F1*F3 bzw. F2*F3 bzw. F1*F2*F3 (ebd). 
Haupteffekte der Kaufplanabfrage betreffen dagegen berichtete Gedanken, und zwar die Gedanken bezüglich der „Ziele bzw. Intentionen“ und der „Alternativenbewertung“ (s. Tab. $12 \&$ 14). In Bezug auf die Alternativenbewertung ließ sich außerdem ein Interaktionseffekt F1*F3, d.h. eine Interaktion mit der „kombinierten Bitte“ um Beobachtung und Befragung (F3) feststellen (ebd). Ein Blick auf die Gruppenmittelwerte lässt erkennen, dass die berichteten Gedanken zu Zielen und Intentionen im Falle einer Kaufplanabfrage seltener sind, es sei denn, dass weder eine Beobachtung noch eine Befragung erbeten wurde. Sie sind besonders selten, wenn im Falle einer Kaufplanabfrage lediglich die Erlaubnis zu einer Beobachtung erbeten wird (s. Tab. 13). Ein anderes Bild zeigt sich bei den Gedanken zur Alternativenbewertung (Tab. 15). Solche Gedanken werden besonders häufig berichtet, wenn im Falle einer Kaufplanabfrage um eine Beobachtung oder um eine Befragung oder um Beides gebeten wird.

\section{Zur Reaktion auf die offene Beobachtung}

Die Varianzanalysen zeigen, dass die erlaubte offene Beobachtung (F2) weder auf das Laufverbalten noch auf das Zuwendungsverbalten signifikante Haupteffekte ausübt (s. Tab. 4, 6, 8 \& 10). Es zeigten sich allerdings drei Interaktionseffekte, und zwar ein „F2*F3-Effekt" auf die Verweildauer, ein Effekt auf die Anzahl der Laufflächenkontakte und ein Effekt auf die Anzahl der Personalkontakte (ebd). Werden eine Beobachtung und eine Nachbefragung erbeten, ist die Verweildauer kürzer im Vergleich zu jenen Fällen, in denen nur eine Beobachtung oder nur eine Befragung erbeten wird (s. Tab. 5). Die Anzahl der Laufflächenkontakte ist gering, wenn weder um Befragung noch um Beobachtung gefragt wird. Sie ist am größten, wenn nur um eine Befragung gebeten wird, größer als dort, wo sowohl um eine Befragung als auch um eine Beobachtung nachgesucht wird (s. Tab. 7). Ähnliche Relationen zeigen sich bezüglich der Personalkontakte (s. Tab. 11).

Ein spezifischer Einfluss der Bitte um Zustimmung zur Beobacbtung auf das Laufverhalten zeigt sich beim Blick auf die Laufflächen-Kontakt-Sequenzen. 
Besucher, die einer Beobachtung zugestimmt haben, betreten den Hauptraum häufiger mit einem Wechsel der Laufrichtung nach rechts. Sie betreten im Nebenraum auch häufiger jenen Bereich, der als etwas abgelegen bezeichnet werden kann.

Und wie stellt sich die Situation bei den berichteten Gedanken dar? Haupteffekte der erlaubten offenen Beobachtung auf die berichteten Gedanken in Sachen Ziele und Intention sowie Alternativenbewertung fanden sich keine, auch keine Interaktionen mit der Bitte um Befragung (F3) und der Kaufplanabfrage (F1) (s. Tab. 12 \& 14).

\section{Zur Reaktion auf die erlaubte, angekündigte Nachherbefragung}

Die Varianzanalysen zeigen, dass sich die Bitte um eine Nachherbefragung (F3) sowohl auf das Laufverhalten als auch auf das Zuwendungsverhalten auswirkt (s. Tab. 4, 6, 8 \& 10) - und zwar auf die Anzahl der Laufflächenkontakte, auf die Häufigkeit der Produktmitnahme und auf die Anzahl der Personalkontakte. Die beobachteten Laufflächenkontakte sind am häufigsten, wenn nur um eine Nachbefragung gebeten wurde, etwas seltener, wenn um Befragung und Beobachtung nachgefragt wurde, und noch seltener, wenn keine der beiden Bitten an die Ladenbesucher herangetragen wurde (s. Tab. 7). Bei der Produktmitnahme zeigt sich ein etwas anderes Bild (s. Tab. 9): Produkte werden am häufigsten mitgenommen, wenn um Beobachtung und Befragung gebeten wurde, und am seltensten, wenn weder um eine Beobachtung noch um eine Befragung gebeten wurde. Die beobachteten Personalkontakte waren am häufigsten, wenn vorab um eine Befragung gebeten wurde, am zweithäufigsten, wenn nur um eine Beobachtung gebeten wurde, und am seltensten, wenn weder um das eine noch um das andere gebeten wurde (s. Tab. 11). Auf die Interaktionseffekte $\mathrm{F} 2 * \mathrm{~F} 3$ bezüglich der Verweildauer, der Laufflächenkontakte und der Personalkontakte (s. Tab. 4, 6 \& 10) wurde bereits oben eingegangen. 
Auswirkungen der angekündigten Beobachtung (F3) auf das Laufverhalten zeigen sich auch bei näherer Betrachtung der einzelnen Laufflächen und der Laufsequenzen. Faktor 3 hat einen Effekt auf die Häufigkeit, mit der zwei Laufflächen, die sich vom Eingang aus gesehen in gerader Laufrichtung befinden, betreten werden. Dasselbe gilt für drei Abfolgen bezüglich der betretenen Laufflächen. Zwei davon bedeuten das Eintreten in den Hauptraum und einen Rechtsschwenk nach einigen Metern, eine davon bedeutet das Wechseln vom Hauptraum in den kleineren Raum beim Verlassen des Hauptraums in Richtung Ausgang - wiederum durch einen Wechsel der Laufrichtung nach rechts.

Tabelle 4: Verweildauer (Min.) - Ergebnisse der Varianzanalyse

\begin{tabular}{lcccc}
\hline Quelle & $d f$ & $F$ & $p$ & $\eta^{2}$ \\
\hline \hline Konstanter Term & 1 & 253,099 &, 000 &, 528 \\
F1 Kaufplanabfrage & 1 &, 293 &, 589 &, 001 \\
F2 Beobachtung erbeten & 1 &, 012 &, 912 &, 000 \\
F3 Befragung erbeten & 1 &, 557 &, 456 &, 002 \\
F1 * F2 & 1 &, 842 &, 360 &, 004 \\
F1 * F3 & 1 &, 016 &, 899 &, 000 \\
F2 * F3 & 1 & $\mathbf{5 , 2 0 2}$ & $\mathbf{, 0 2 3 *}$ & $\mathbf{, 0 2 3}$ \\
F1 * F2 * F3 & 1 &, 005 &, 944 &, 000 \\
Fehler & 226 & & & \\
\hline
\end{tabular}

$* \mathrm{p}<.05 ; * * \mathrm{p}<.01 ; * * * \mathrm{p}<.001$

Tabelle 5: Verweildauer (Min.) - Mittelwerte und Standardabweichungen

\begin{tabular}{cccc}
$\begin{array}{c}\text { Befragung und } \\
\text { Beobachtung nicht } \\
\text { erbeten }\end{array}$ & $\begin{array}{c}\text { Beobachtung } \\
\text { erbeten }\end{array}$ & $\begin{array}{c}\text { Befragung } \\
\text { erbeten }\end{array}$ & $\begin{array}{c}\text { Befragung und } \\
\text { Beobachtung } \\
\text { erbeten }\end{array}$ \\
\hline
\end{tabular}

Keine

Kaufplanabfrage $\quad 6,06(4,53) \quad 7,48(4,83) \quad 8,79(7,46) \quad 5,89(3,51)$

Kaufplanabfrage $\quad 5,67(3,38) \quad 8,64(13,24) \quad 8,50(6,78) \quad 7,41(7,51)$


Haupteffekte der angekündigten Beobachtung auf die berichteten Kognitionen fanden sich keine (s. Tab. 12 \& 14). Allerdings konnte bezüglich der Alternativenbewertung ein Interaktionseffekt F1*F3 festgestellt werden, eine Interaktion mit der Kaufplanabfrage (s. Tab. 14). Auch auf diesen Effekt wurde oben bereits eingegangen.

Tabelle 6: Anzabl der Laufflächenkontakte - Ergebnisse der Varianzanalyse

\begin{tabular}{lcccc} 
Quelle & $d f$ & $F$ & $p$ & $\eta^{2}$ \\
\hline \hline Konstanter Term & 1 & 1079,050 &, 000 &, 823 \\
F1 Kaufplanabfrage & 1 &, 256 &, 613 &, 001 \\
F2 Beobachtung erbeten & 1 &, 030 &, 864 &, 000 \\
F3 Befragung erbeten & 1 & 3,199 &, $075^{\mathrm{T}}$ &, 014 \\
F1* F2 & 1 &, 700 &, 404 &, 003 \\
F1* F3 & 1 &, 268 &, 605 &, 001 \\
F2* F3 & 1 & $\mathbf{4 , 0 6 8}$ &, $045 *$ &, 017 \\
F1* F2* F3 & 1 &, 056 &, 813 &, 000
\end{tabular}

Fehler 232

$* \mathrm{p}<.05 ; * * \mathrm{p}<.01 ; * * * \mathrm{p}<.001$

Tabelle 7: Anzabl der Laufflächenkontakte - Mittelwerte und Standardabweichungen

\begin{tabular}{lcccc}
\hline & $\begin{array}{c}\text { Befragung und } \\
\text { Beobachtung } \\
\text { nicht erbeten }\end{array}$ & $\begin{array}{c}\text { Beobachtung } \\
\text { erbeten }\end{array}$ & $\begin{array}{c}\text { Befragung } \\
\text { erbeten }\end{array}$ & $\begin{array}{c}\text { Befragung und } \\
\text { Beobachtung } \\
\text { erbeten }\end{array}$ \\
\hline \hline $\begin{array}{l}\text { Keine } \\
\text { Kaufplanabfrage }\end{array}$ & $29,67(9,18)$ & $35,52(16,29)$ & $36,07(19,42)$ & $34,45(14,56)$ \\
Kaufplanabfrage & $30,90(12,38)$ & $34,23(21,55)$ & $40,47(17,90)$ & $34,34(15,75)$ \\
\hline
\end{tabular}


Tabelle 8: Häufigkeit der „Produktmitnahme“- Ergebnisse der V arianzanalyse

\begin{tabular}{lcccc} 
Quelle & $d f$ & $F$ & $p$ & $\eta^{2}$ \\
\hline \hline Konstanter Term & 1 & 98,680 &, 000 &, 298 \\
F1 Kaufplanabfrage & 1 &, 944 &, 332 &, 004 \\
F2 Beobachtung erbeten & 1 & 2,271 &, 133 &, 010 \\
F3 Befragung erbeten & 1 & 2,722 &, 100 &, 012 \\
F1 * F2 & 1 &, 906 &, 342 &, 004 \\
F1* F3 & 1 &, 164 &, 686 &, 001 \\
F2 * F3 & 1 & 1,425 &, 234 &, 006 \\
F1 * F2 * F3 & 1 &, 008 &, 928 &, 000
\end{tabular}

Fehler 232

$* \mathrm{p}<.05 ;{ }^{* *} \mathrm{p}<.01 ; * * * \mathrm{p}<.001$

Tabelle 9: Häufigkeit der „Produktmitnahme“-Mittelwerte und Standardabweichungen

$\begin{array}{cccc}\text { Befragung und } & \text { Beobachtung } & \text { Befragung } & \text { Befragung und } \\ \text { Beobachtung } & \text { erbeten } & \text { erbeten } & \text { Beobachtung } \\ \text { nicht erbeten } & & & \text { erbeten }\end{array}$

Keine

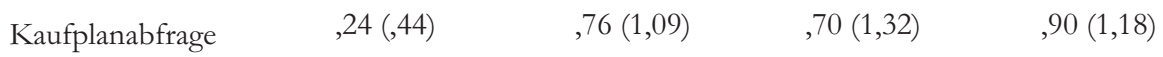

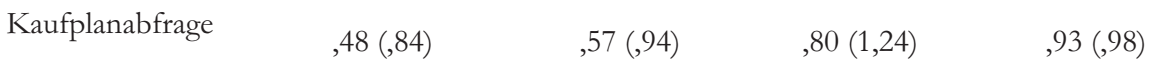

Tabelle 10: Anzahl der Kontakte mit dem Verkaufspersonal-Ergebnisse der V arianzanalyse

\begin{tabular}{lcccc}
\hline Quelle & $d f$ & $F$ & $p$ & $\eta^{2}$ \\
\hline \hline Konstanter Term & 1 & 75,501 &, 000 &, 246 \\
F1 Kaufplanabfrage & 1 &, 839 &, 361 &, 004 \\
F2 Beobachtung erbeten & 1 &, 078 &, 780 &, 000 \\
F3 Befragung erbeten & 1 & 3,209 &, $075^{\mathrm{T}}$ &, 014 \\
F1*F2 & 1 &, 009 &, 926 &, 000 \\
F1* F3 & 1 &, 084 &, 772 &, 000 \\
F2* F3 & 1 & $\mathbf{1 3 , 7 8 5}$ & $\mathbf{, 0 0 0 * * *}$ & $\mathbf{0 5 6}$ \\
F1* F2* F3 & 1 & 1,240 &, 267 &, 005 \\
Fehler & 232 & & & \\
\hline
\end{tabular}

${ }^{*} \mathrm{p}<.05 ;{ }^{* *} \mathrm{p}<.01 ;{ }^{* * *} \mathrm{p}<.001$ 
Tabelle 11: Anzabl der Kontakte mit dem Verkaufspersonal - Mittelwerte und Standardabweichungen

\begin{tabular}{lcccc}
\hline & $\begin{array}{c}\text { Befragung und } \\
\text { Beobachtung } \\
\text { nicht erbeten }\end{array}$ & $\begin{array}{c}\text { Beobachtung } \\
\text { erbeten }\end{array}$ & $\begin{array}{c}\text { Befragung } \\
\text { erbeten }\end{array}$ & $\begin{array}{c}\text { Befragung und } \\
\text { Beobachtung } \\
\text { erbeten }\end{array}$ \\
\hline \hline $\begin{array}{l}\text { Keine } \\
\text { Kaufplanabfage }\end{array}$ &, $21(, 55)$ &, $83(1,28)$ & $1,17(1,68)$ &, $45(, 78)$ \\
Kaufplanabfrage &, $27(, 83)$ &, $60(1,03)$ &, $83(, 98)$ &, $45(1,08)$ \\
\hline
\end{tabular}

Tabelle 12: Anzabl der berichteten Kognitionen zu "Zielen \& Intention“ - Ergebnisse der Varianzanalyse

\begin{tabular}{lcccc}
\hline Quelle & $d f$ & $F$ & $p$ & $\eta^{2}$ \\
\hline \hline Konstanter Term & 1 & 153,152 &, 000 &, 406 \\
F1 Kaufplanabfrage & 1 & $\mathbf{7 , 6 6 3}$ & $\mathbf{, 0 0 6 * *}$ & $\mathbf{, 0 3 3}$ \\
F2 Beobachtung erbeten & 1 & 2,148 &, 144 &, 009 \\
F3 Befragung erbeten & 1 &, 106 &, 745 &, 000 \\
F1 * F2 & 1 & 2,652 &, 105 &, 012 \\
F1 * F3 & 1 &, 663 &, 416 &, 003 \\
F2 * F3 & 1 &, 663 &, 416 &, 003 \\
F1 * F2 * F3 & 1 & 2,652 &, 105 &, 012 \\
Fehler & 224 & & & \\
\hline
\end{tabular}

$* \mathrm{p}<.05 ; * * \mathrm{p}<.01 ; * * * \mathrm{p}<.001$ 
Tabelle 13: Anzabl der berichteten Kognitionen zu „Zielen \& Intention“ - Mittelwerte und Standardabweichungen

\begin{tabular}{lcccc}
\hline & $\begin{array}{c}\text { Befragung und } \\
\text { Beobachtung } \\
\text { nicht erbeten }\end{array}$ & $\begin{array}{c}\text { Beobachtung } \\
\text { erbeten }\end{array}$ & $\begin{array}{c}\text { Befragung } \\
\text { erbeten }\end{array}$ & $\begin{array}{c}\text { Befragung und } \\
\text { Beobachtung } \\
\text { erbeten }\end{array}$ \\
\hline \hline $\begin{array}{l}\text { Keine } \\
\text { Kaufplanabfrage }\end{array}$ &, $72(, 882)$ & $0,83(, 96)$ & $0,86(0,875)$ &, $79(, 82)$ \\
Kaufplanabfrage &, $86(, 92)$ & $0,28(0,53)$ & $0,48(, 785)$ & $0,41(, 57)$ \\
\hline
\end{tabular}

Mit dem Blick auf sämtliche Varianzanalysen lässt sich noch Folgendes festhalten und herausstellen: Zu den Variablen, bei denen weder Haupteffekte noch Interaktionseffekte der drei Faktoren festgestellt werden konnten, zählen die Anzahl der Zuwendungen „Schauen“, die Anzahl der Zuwendungen „Anfassen“, die Anzahl der Zuwendungen „Mitnahme“, die Anzahl der „ungeplanten Käufe“ und die Anzahl aller berichteten Kognitionen. Damit relativiert sich das Bild bezüglich der Reaktivität einer Kaufplanabfrage sowie eine offenen Beobachtung und einer angekündigten Nachbefragung von Ladenbesuchern nicht unwesentlich.

Tabelle 14: Anzabl der berichteten Kognitionen zur „Alternativenbewertung"-Ergebnisse der Varianzanalyse

\begin{tabular}{lcccc}
\hline Quelle & $d f$ & $F$ & $p$ & $\eta^{2}$ \\
\hline \hline Konstanter Term & 1 & 111,727 &, 000 &, 333 \\
F1 Kaufplanabfrage & 1 & $\mathbf{6 , 0 5 9}$ & $\mathbf{, 0 1 5 *}$ & $\mathbf{, 0 2 6}$ \\
F2 Beobachtung erbeten & 1 &, 084 &, 772 &, 000 \\
F3 Befragung erbeten & 1 &, 021 &, 885 &, 000 \\
F1 * F2 & 1 & 2,097 &, 149 &, 009 \\
F1 * F3 & 1 & 4,717 &, $031 *$ &, 021 \\
F2 * F3 & 1 &, 755 &, 386 &, 003 \\
F1 * F2 * F3 & 1 &, 755 &, 386 &, 003 \\
Fehler & 224 & & & \\
\hline
\end{tabular}

$* \mathrm{p}<.05 ;{ }^{* *} \mathrm{p}<.01 ;{ }^{* * *} \mathrm{p}<.001$ 
Tabelle 15: Anzabl der berichteten Kognitionen zur „Alternativenbewertung“- Mittelwerte und Standardabweichungen

\begin{tabular}{lcccc}
\hline & $\begin{array}{c}\text { Befragung und } \\
\text { Beobachtung } \\
\text { nicht erbeten }\end{array}$ & $\begin{array}{c}\text { Beobachtung } \\
\text { erbeten }\end{array}$ & $\begin{array}{c}\text { Befragung } \\
\text { erbeten }\end{array}$ & $\begin{array}{c}\text { Befragung und } \\
\text { Beobachtung } \\
\text { erbeten }\end{array}$ \\
\hline \hline Keine &, $69(, 93)$ &, $55(, 87)$ &, $41(, 63)$ &, $28(, 59)$ \\
Kaufplanabfrage &, $45(, 63)$ &, $86(1,15)$ &, $90(1,14)$ &, $90(1,08)$ \\
Kaufplanabfrage & $, 608)$ \\
\hline
\end{tabular}

\section{(3) Zusammenfassung und Interpretation der Ergebnisse}

Als Ergebnisse eines ersten multifaktoriellen Feldexperiments zur Reaktivität der Verhaltensforschung am POS lässt sich festhalten, dass die Kaufplanabfrage, die Bitte um Beobachtung und die die Bitte um ein Interview nach dem Ladenbesuch sowie die jeweilige Zustimmung der Probanden deren Lauf- und Zuwendungsverhalten sowie deren Gedanken durchaus beeinflussen. Allerdings werden nicht alle untersuchten Konkretisierungen dieses Verhaltens beeinflusst. Auch sind nicht alle Treatmentwirkungen bei den beeinflussten Sachverhalten gleich stark ausgeprägt, auch nicht die Interaktionseffekte dieser Treatments. Unseren Befunden folgend lässt eine Kaufplanabfrage nur kognitive Reaktionen erwarten, die offene Beobachtung keine kognitiven Reaktionen, dafür aber Einflüsse beim Lauf- und Zuwendungsverhalten, allerdings keine Haupteffekte, sondern nur Interaktionseffekte. Die Ankündigung einer Befragung und die entsprechende Zustimmung seitens der Ladenbesucher zeigen dagegen mehrere Haupteffekte und ganz verschiedene Interaktionseffekte auf deren Lauf- und Zuwendungsverhalten - auf die Anzahl der Laufflächenkontakte, auf die Anzahl der Produktmitnahme und die Anzahl der Personalkontakte. Interaktionseffekte zeigen sich auch bezüglich der berichteten Kognitionen. Solche Befunde sprechen letztlich für eine differenzierte, aber auch für eine fortgesetzte Analyse der Reaktivität in der Verhaltensforschung am POS. Künftige Studien sollten jedenfalls eine bessere Methode bei der Erfassung kognitiver Prozesse einsetzen - z.B. die 
gestützte Gedankenrekonstruktion. Denn in der berichteten Studie mussten die Probanden die Gedanken beim Ladenbesuch in Nachhinein erinnern und angeben, was das Gedächtnis nicht selten überfordert haben dürfte. Ein Hinweis in dieser Richtung liefert die durchweg geringe Anzahl der berichteten Gedanken - ein Umstand, der die Aussagekraft unserer Befunde zur kognitiven Reaktivität begrenzt.

\subsection{Eine Studie zur Reaktion von Ladenbesuchern auf die Denke-Laut- Methode und die videogestützte Gedankenrekonstruktion}

Büttner (2008) führte am Institut für Marketing und Handel der Universität Göttingen eine Studie durch, die auch Aufschlüsse zur Reaktion von Ladenbesuchern auf den Einsatz der „Denke-Laut-Methode“ (DLM) und der „Videogestützten Gedanken-Rekonstruktion“ (VGR) liefert.

\section{(1) Fragestellung und Anlage der Studie}

In einem Feldexperiment sollten die DLM und die VGR untereinander und dann auch mit einer Kontrollgruppe verglichen werden, in der keine der beiden Methoden zum Einsatz kommt. Außerdem wurde eine unterschiedliche Besuchsmotivation induziert, um Seeker und Surfer vergleichen zu können (Büttner 2008 S.130f.). Die DLM-Gruppe trug eine unauffällige Kopfkamera, die es erlaubte, nicht nur das Zuwendungsverhalten zu erfassen, sondern auch das erbetene „laute Denken“. Die VGR-Gruppe trug ebenfalls eine unauffällige Kopfkamera, die das Blickfeld als dynamisches Geschehen aufzeichnete, um das so erstellte Blickfeldvideo anschließend als Erinnerungsstütze bei der Gedankenrekonstruktion heranziehen zu können. In der Kontrollgruppe kamen keine Kopfkameras zum Einsatz und es wurde auch nicht darum gebeten, die Gedanken $\mathrm{zu}$ artikulieren (ebd). Die Erhebung fand in einem innerstädtischen Elektrofachmarkt statt.

In Bezug auf die Reaktivität der Verhaltensforschung am POS interessierte die Frage, wie die Probanden bei ihrem Ladenbesuch auf die DLM und auf die VGR reagieren und welche der beiden Methoden bei der Erfassung kognitiver Prozesse 
vorzuziehen sei (S.122f.). Vor dem Hintergrund einschlägiger Theorien wurden folgende Effekte erwartet (S.129): (1) Das Tragen einer Kopfkamera erhöht die Selbstaufmerksamkeit und reduziert den Einkaufsnutzen, das Einkaufsvergnügen und die Anzahl ungeplanter Käufe. (2) Lautes Denken bindet kognitive Kapazität und reduziert deshalb den Einkaufsnutzen, das Einkaufsvergnügen und die Anzahl ungeplanter Käufe. Zu den Kognitionen der Seeker und der Surfer wurde ebenfalls eine Hypothese formuliert (3). Die Gedanken der Seeker kreisen vornehmlich um die Realisierung der Kaufziele, die Gedanken der Surfer vornehmlich um die Wünschbarkeit und Realisierbarkeit von Produktkäufen (S. 130).

\section{(2) Auswertung und Darstellung der Ergebnisse}

Die Befunde bzw. deren varianzanalytische Auswertung zeigen, dass die private Selbstaufmerksamkeit beim Einsatz der DLM und der VGR nicht größer ist als in der Kontrollgruppe, wohl aber die öffentliche Selbstaufmerksamkeit. Unterschiede zwischen der DLM-Gruppe und VGR-Gruppe zeigen sich aber auch hier nicht (S. 152f.).

Der Einkaufsnutzen, der in der Seeker-Gruppe erfasst wurde und zwar über die Effektivität und Effizienz bei der Lösung der Kaufaufgabe, zeigte bezüglich des DLM- und VGR-Einsatzes keine (signifikanten) Unterschiede. Doch war die durchschnittliche Aufenthaltsdauer in der DLM- und in der VGR-Gruppe länger als die der Kontrollgruppe (S.153f.). Das Einkaufsvergnügen variierte hier ebenfalls. Die positiven Emotionen waren in der Kontrollgruppe zahlreicher als in den beiden anderen Gruppen, negative Emotionen dagegen seltener - aber lediglich auf das Segment der Surfer bezogen (S. 157).

Die Wahrscheinlichkeit ungeplanter Käufe im Segment der Seeker ist in der DLM- und VGR-Gruppe nur dann geringer als in der Kontrollgruppe, wenn die Surfer betrachtet werden. Auf die Seeker trifft der erwartete Unterschied bezüglich der Neigung zu ungeplanten Käufen nicht zu. Und für die Surfer zeigt sich 
außerdem, dass die Neigung zu ungeplanten Käufen in der DLM-Gruppe deutlich geringer ausfällt als in der VGR-Gruppe (S. 162f.).

\section{(3) Zusammenfassung und Interpretation der Ergebnisse}

Die beiden analysierten Methoden der Gedankenerfassung - die DLM- und die VGR-Methode - erzeugen, wie erwartet, ebenfalls Reaktivität. Sie steigern zunächst die (öffentliche) Selbstaufmerksamkeit, was sich vor allem aus dem Umstand erklären dürfte, dass die Offenlegung von Gedanken eine unübliche, ungewohnte Tätigkeit darstellt und in einem quasi-öffentlichem Raum und zumindest dem Forscher gegenüber erfolgen muss. Beide Methoden bewirken außerdem eine längere Aufenthaltsdauer, bedingt durch die Aufgabe der Gedankenartikulation einerseits und die Behinderung der eigentliche Kauf- bzw. Suchaufgabe andererseits. Dies beeinträchtigt auch das Kauf- bzw. Shoppingvergnügen und führt bei den Ladenbesuchern ohne Kaufabsicht (den Surfern) dazu, dass auch die Neigung zu ungeplanten Käufe geringer ausgeprägt ist. Damit bestätigt sich die in anderen Studien immer wieder festgestellte positive Korrelation zwischen dem Einkaufsvergnügen einerseits und dem Umfang der ungeplanten Produktkäufe andererseits.

\subsection{Zusammenfassung der wichtigsten Ergebnisse}

Den derzeitigen Stand der Forschung zusammenfassend lässt sich Folgendes festhalten: Wie erwartet ist beim Einsatz der Befragungsmethode und der Beobachtungsmethode allein schon aufgrund ihrer Ankündigung bzw. aufgrund der Bitte um Teilnahme mit Reaktivität zu rechnen. Dies gilt auch für die Abfrage von Kaufplänen vor dem Betreten des Geschäfts. Ladenbesucher, die offen beobachtet werden, neigen dazu, verstärkt in die Rolle des Käufers zu schlüpfen und dementsprechend auch mehr zu kaufen. Ihr Lauf- und Zuwendungsverhalten änderte sich in einer der Studien nicht, in der anderen dagegen wohl: nicht nur die Laufrichtung, sondern auch die Zuwendung am Regal. Ähnliches gilt für die Ankündigung einer Nachher-Befragung. Wird ein Ladenbesucher um die Erlaubnis 
zur Beobachtung und um die Teilnahme an einer Nachher-Befragung gebeten, sind die genannten Effekte ebenfalls festzustellen, auch eine Art „Potenzierung“ der Reaktivität, die sich in einer verstärkten Mitname von Ware äußert. Reaktivität ist wie erwartet - besonders ausgeprägt beim Einsatz der DLM und der VGR. Beide Verfahren steigern die (öffentliche) Selbstaufmerksamkeit, führen zu einer längeren Aufenthaltsdauer und zu einer Beeinträchtigung des Einkaufsvergnügens. Besucher ohne Kaufabsicht zeigen außerdem eine geringere Bereitschaft, Produkte dennoch zu kaufen. Damit bestätigen erste Studien nicht nur, dass die Verhaltensforschung am POS mit Reaktivität rechnen muss, sondern auch, dass diese Effekte bei unterschiedlichen Messinstrumenten und in unterschiedlichen Geschäften unterschiedlich ausgeprägt sein können.

\section{Folgerungen für die Verhaltensforschung am Point-of-Sale}

Wenn es darum geht, die externe Validität und damit die Generalisierbarkeit von Befunden einer Verhaltensforschung am POS zu sichern oder zu verbessern, impliziert dies die Kontrolle, aber auch die Reduzierung der verschiedenen denkbaren Reaktivitätseffekte. Dies gilt für Studien, die von kommerziellen Instituten und unternehmensinternen Stellen durchgeführt werden, ebenso wie für wissenschaftliche Untersuchungen.

Die Kontrolle beginnt mit einem Blick auf die möglichen Effekte. Einige seien hier noch einmal erwähnt, um die Vielfalt solcher Effekte anzudeuten: die Beeinflussung der Wahrnehmung, vor allem deren Sensibilisierung und deren Ausrichtung (Selektivität) für die Ladenumwelt und für die eigene Person, so z.B. die Steigerung der Selbstaufmerksamkeit, die Ablenkung von ursprünglich gehegten Kaufabsichten, der erhöhte Zeitaufwand beim Ladenbesuch, die Veränderung der Motivation und die Entwicklung neuer Kaufideen, die Übernahme einer bestimmten, im Alltag keineswegs üblichen Rolle, so z.B. die Rolle des besonders umsichtigen Käufers oder die Rolle eines kaufkräftigen Verbrauchers, um ein gutes Bild von sich abzugeben, sowie die studienbedingte Einbuße an Einkaufserleben bzw. Einkaufsfreude. Die Bestimmung derartiger Reaktivitäten erfordert nicht nur 
Erfassung der relevanten Variablen, sondern auch den Vergleich mit Kontrollgruppen, in denen keine Messungen angekündigt werden und außerdem dafür Sorge getragen wird, dass keine anderen Hinweise auf anstehende Messungen die Zielgruppe erreichen.

Eine zweite Möglichkeit besteht darin, unvermeidliche Reaktivitätseffekte so gut wie möglich zu begrenzen. Für bestimmte Messinstrumente werden gelegentlich entsprechende Vorschläge unterbreitet. Für Beobachtungsstudien empfehlen einige Autoren vor allem die gezielte Auswahl, Ausbildung und Überwachung der eingesetzten Beobachter in Bezug auf erwünschte und unerwünschte Verhaltensweisen im Beobachtungsgeschehen; diese Autoren verweisen z.T. auch auf die Notwendigkeit, die Interaktion im Beobachtungsgeschehen zu kontrollieren (z.B. Kent \& Foster 1977 pp.314ff., Wildman \& Erickson 1977, Spool 1978, Haynes \& Horn 1982 pp.381ff., McCall 1984 S.274f.). Wichtig ist vor allem, dass die verdeckte Beobachtung in der Kontrollgruppe gelingt und dass bei deren Ankündigung keine Erwünschtheitseffekte (demand effects) erzeugt werden. Bei anderen Instrumenten gilt einiges analog. So ist bei der Ankündigung einer Befragung, einer DLM oder einer VGR z.B. darauf zu achten, dass so wenig wie möglich über den Gegenstand der Befragung und die ggf. entwickelten Hypothesen ausgesagt wird. Und wenn es der Rechtsrahmen erlaubt, sollten bestimmte Erhebungen, die keinerlei personenbezogene Daten erzeugen, so z.B. die Ortung von Einkaufswagen, gar nicht erst kommuniziert werden.

Sollte es die künftige Forschung erlauben, den Einfluss bestimmter Größen auf Reaktivitätseffekte zu bestimmen, ließen sich entsprechende Einschätzungen als Korrekturgrößen verwenden. Auf diese Weise könnte dann mit weniger Aufwand der Forderung Rechnung getragen werden, dass die Verhaltensforschung am POS die Art und die Stärke von Reaktivitätseffekten bei der Auswertung und Interpretation von Forschungsergebnissen berücksichtigen muss. Bis dahin ist noch ein weiter Weg zu beschreiten, denn die Forschung ist heute noch weit davon 
entfernt, sagen zu können, wann bei welcher Forschungsmethode, bei welchem Forschungsgegenstand, bei welcher Population und in welcher Situation bzw. in welchem Geschäft welche Reaktivitätseffekte zu erwarten sind.

\section{Literatur}

Adler, P. A. \& Adler, P. (1994).

Oberservational Techniques, in: N. K. Denzin \& Y. S. Lincoln (Eds.), Handbook of Qualitative Research, Thousand Oaks: Sage Publications, pp. 377-392

Bettman, J. R. \& Zins, M. A. (1977).

Constructive Processes in Consumer Choice, in: Journal of Consumer Research, Vo. 4 (1977), No 2, pp. 75-85

Biggs, S. F., Rosman, A. J., \& Sergenian, G. K. (1993).

Methodological Issues in Judgement and Decision-Making Research: Concurrent Verbal Protocol Validity an Simultaneous Traces of Process, in: Journal of Behavioral Decision Making, Vol. 6 (1993), No 3, pp. 187-206

Büttner, O. (2008).

Die Untersuchung einkaufsbegleitender kognitiver Prozesse am Point of Sale- Eine Arbeit zur Qualität von Datenerhebungsmethoden der Konsumentenforschung, Dissertationsschrift, Universität Göttingen, Wirtschaftswissenschaftliche Fakultät

Chandon, P., Morwitz, V. G., \& Reinartz, W. J. (2004).

The Short- and Long-term Effects of Measuring Intent to Repurchase, in: Journal of Consumer Research, Vol. 31 (2004), No 3, pp. 566-572

Cobb, C. J. \& Hoyer, W. D. (1986).

Planned Versus Impulse Purchase Behavior, in: Journal of Retailing, Vol. 60 (1986);

No 4, pp. 384-409

Dholakia, U. M. \& Morwitz, V. G. (2002).

The Scope and Persistence of Mere Measurement Effects: Evidence From a Field Study of Customer Satisfaction Measurement, in: Journal of Consumer Research, Vol. 29 (2002), No 2, pp. 159-167

Ericsson, K. A. \& Simon, H. A. (1993).

Protocol Analysis: Verbal Reports as Data, rev. Ed., Cambridge, MA: MIT Press 
Haynes, S. N. \& Horn, W. F. (1982).

Reactivity in Behavioral Observation: A Review, in: Behavioral Assessment, Vol. (1982), No 4, pp. 369-385

Hoc, J. M. \& Leplat, J. (1983).

Evaluation of Different Modalities of Verbalization in a Sorting Task, in: International Journal of Man-Machine Studies, Vol. 18 (1983), No 3, pp. 283-306

Johnson, S. M. \& Bolstad, O. D. (1973).

Methodological Issues in Naturalistic Observation: Some Problems and Solutions for Field Research, in: L. A. Hamerlynck, L. C. Handy, \& E. J. Mash (Eds.), Behavior Change: Methodology Concepts and Practise, Champaign, IL: Research Press, pp. 7-67

Kent, R. N. \& Foster, S. L. (1977).

Direct Observational Procedures: Methodological Issues in Naturalistic Settings, in: A. R. Ciminero, K. S. Calhoun, \& H. S. Adams (Eds.). Handbook of Behavioural Assessment, New York NY: Wiley, pp. 279-328

Lesser, J. A. \& Hughes, M. A. (1986).

Towards a Typology of Shoppers, in: Business Horizons, Nov./Dec. 1986, pp. 5662

McCall, G. J. (1984).

Systematic Field Observation, in: Annual Review of Sociology, Vol. 10 (1984), pp. 263-282

Orne, M. T. (1962).

On the Social Psychology of the Psychological Experiment: With Particular Reference to Demand Characteristics and their Implications, in: American Psychologist, Vol. 17 (1962), pp. 776-783

Paulhus, D. L. (1991).

Measurement and Control of Response Biases, in: J. P. Robinson, P. R. Shaver, \& L. S. Wrightsmann (Eds.), Measures of Personality and Social Psychological Attitudes, Vol. 1, San Diego: Academic, pp. 17-59

Prasad, V. K. (1975).

Unplanned Buying in Two Retail Settings, in: Journal of Retailing, Vol. 51 (1975), No 3, pp. 3-12 
Rosenthal, R. (1966).

Experimenter Effects in Behavioral Research. New York: Appleton-Century-Crofts

Ruiz, J.-P., Chebat, J.-C. \& Hansen, P. (2004).

Another Trip to the Mall: A Segmentation Study of Customers Based on Their Activities, in: Journal of Retailing and Consumer Services, Vol. 11 (2004), pp. 333 350

Russo, J. E., Johnson, E. J. \& Stephens, D. (1989).

The Validity of Verbal Protocols, in: Memory \& Cognition, Vol. 17 (1989), No 6, pp. 759-769

Smith, R. L., McPhail, C. \& Pickens, R. G. (1975).

Reactivity to Systematic Observation with Film: A Field Experiment, in: Sociometry, Vol. 38 (1975), No 4, pp. 536-550

Spool, M. D. (1978).

Training Programs for Observers of Behavior: A Review, in: Personnel Psychology, Vol. 31 (1978), pp. 853-888

Van denHaak, M. J., de Jong, M. D. T. \& Schellens, J. P. (2003).

Retrospective vs. Concurrent Think-Aloud Protocols: Testing the Usability of an Online Library Catalogue, in: Behavior \& Information technology, Vol. 22 (2003), No 5, pp. 339-351

Webb, E. J., Campbell, D. T., Schwartz, R. C. \& Sechrest, L. (1966).

Unobtrusive Measures: Nonreactive Research in the Social Sciences. Chicago: University of Chicago Press

Wildman, B. G., \& Erickson, M. T. (1977).

Methodological Problems in Behavioral Observation, in: J. D. Cone \& R. P. Hawkins (Eds.). Behavioral Assessment: New Directions in Clinical Psychology, New York: Brunner \& Mazel, pp. 255-273 
G

Die abgestufte Verhaltensforschung am Point-of-Sale 


\section{Einleitung}

Das Verhalten der Nachfrager am POS ist von zentraler Bedeutung für deren Kauferfolg und für den Absatzerfolg der Anbieter. Deshalb haben auch jene Studien, die Aufschlüsse über das Verhalten der Nachfrager am POS liefern, großes Gewicht. Es geht somit um die Aussagekraft dieser Studien und damit für die Eignung der verschiedenen Messinstrumente. Diese Aussagekraft wurde an anderer Stelle behandelt. Dabei wurde auch auf die mögliche Kombination verschiedener Messinstrumente eingegangen.

Angesichts der unterschiedlichen Aussagekraft einzelner Forschungsmethoden und angesichts des Aufwandes, der mit einer Erfassung des Nachfragerverhaltens am POS verbunden ist, stellt sich auch die Frage nach einer Methodenkombination der ganz anderen Art, nämlich die Frage nach der Möglichkeit, unterschiedliche Methode in einer sinnvollen Abfolge einzusetzen. Dieser Frage ist in der Fachliteratur und wohl auch in der Praxis bislang keinerlei Beachtung geschenkt worden.

Im Folgenden wollen wir uns der Frage zuwenden, wie eine sinnvolle Abfolge von Forschungsvorhaben am POS im Zeitablauf aussehen könnte. Dabei werden wir uns auf den stationären Handel konzentrieren, in dem noch immer die meisten Verbraucher ihre Einkäufe tätigen. Der Vorschlag einer sinnvollen Abfolge soll sich dabei nicht nur auf allgemeine methodenbezogene und aufwandsbezogene Überlegungen stützen, sondern auch auf die Darstellung einer möglichen Sequenz und die zu erwartenden Befunde anhand konkreter Beispiele. Zunächst wollen wir uns jedoch ganz kurz den wichtigsten Dimensionen des Besucherverhaltens und den Möglichkeiten ihrer Konkretisierung und ihrer Messung zuwenden. Daran anschließend wird aufgezeigt, wie im Rahmen eines abgestuften Vorgehens die Aussagekraft einer Verhaltensforschung am POS gesteigert und der erforderliche Aufwand begrenzt werden kann. 


\section{Dimensionen des Verhaltens von Ladenbesuchern und die Möglich- keiten ihrer Erfassung}

Zentrale Dimensionen des Verhaltens von Ladenbesuchern sind das Laufverhalten, das Zuwendungsverhalten und die kognitiven Prozesse. Das Laufverhalten entscheidet vor allem über die Anzahl der Kontakte mit dem Angebot und das Zuwendungsverhalten vor allem über die Qualität dieser Kontakte. Die kognitiven Prozesse bestimmen ebenfalls die Qualität dieser Kontakte, stehen aber auch für die vor Ort getroffenen Kaufentscheidungen und für das Kauferleben, und somit für den intendierten Kontakt- bzw. Kauferfolg.

\section{(1) Das Laufverhalten und dessen Erfassung}

Das Laufverhalten der Ladenbesucher, häufig auch als Kundenlauf bezeichnet, lässt sich unterschiedlich operationalisieren. Eine mögliche Konkretisierung besteht darin, den Kundenlauf als eine Sequenz der Laufflächenkontakte zu erfassen. Je größer die dabei definierten Flächen sind, desto grober ist die Operationalisierung des Kundenlaufs. Eine andere Konkretisierung kann an der Einteilung des Angebotes, z.B. an Regalen mit unterschiedlichen Angeboten, festmachen. In einem solchen Falle hätte der Kundelauf Auskunft zu geben, in welcher Reihenfolge welche Regale aufgesucht wurden bzw. in welcher Reihenfolge an welchen Regalen vorbeigegangen wurde. Wichtig wäre dabei die Konkretisierung des „Am Regal vorbeigehen“. Dabei wäre nicht nur an eine Mindestentfernung zum jeweiligen Regal zu denken, sondern auch an die visuelle Zuwendung.

Bei der Erfassung des Laufverhaltens kommen mehrere Methoden in Frage: (1) die Registrierung über Ortungssysteme, z.B. RFID-Systeme, und über installierte Raumkameras, (2) die klassische Beobachtung, verdeckt oder offen, und (3) die Befragung, sei es die Nachherbefragung, sei es die parallele Erfassung im Rahmen der sog. Handle-Laut-Methode. Ortungs- und Videosysteme können auf diverse Schwierigkeiten stoßen: fehlende Anonymität, rechtliche Barrieren, Lücken beim raum- und etagenübergreifenden Tracking sowie die fehlende Akzeptanz bei den 
Ladenbesuchern. Das anonyme Tracking wäre bei RFID-Systemen machbar, die entsprechend ausgerüstete Einkaufskörbe und Einkaufswagen verfolgen zu können. Das Personentracking müsste auf entsprechend ausgerüstete Kundenoder Besucherkarten setzen, die der Besucher mit sich trägt. Die klassische Beobachtung ist als verdeckte Beobachtung mit der Gefahr verbunden, bemerkt zu werden und zu verärgern, und als offene Beobachtung mit dem Problem, dass nicht alle Besucher einer Beobachtung zustimmen. Bei der Befragung der Besucher zum soeben realisierten Laufverhalten kommt aber noch hinzu, dass mit mehr oder weniger großen Erinnerungslücken gerechnet werden muss.

Wenn Laufsequenzen erfasst werden konnten, sind diese auch entsprechend auszuwerten, nämlich als Sequenzen von Flächen- oder Regalkontakten. Hierbei können geeignete Distanzmaße zum Einsatz kommen, wenn es darum geht, Sequenzen $\mathrm{zu}$ vergleichen und das Ergebnis derartiger Vergleiche in metrische Angaben zu überführen. Ist dies erfolgt, können sog. Sequenz-Cluster gesucht und bestimmt werden, die dann auf sog. Laufmuster hinweisen.

\section{(2) Das Zuwendungsverhalten und dessen Erfassung}

Das Laufen wird zum Vorbeilaufen, wenn die Zuwendung zum Angebot oder zum Personal ausbleibt. Das Laufen kann aber auch mit einem mehr oder weniger ungerichteten Schauen einhergehen. Stärker ausgerichtete Zuwendungen liegen dann vor, wenn ein bestimmtes Angebot angeschaut, ggf. in die Hand genommen und ggf. auch mitgenommen wird. Analoges gilt bei der Inanspruchnahme von Auskunfts- oder Servicepersonen. Bezogen auf diese Arten der Zuwendung zu ausgelegten Waren lässt sich eine Rangordnung vorstellen: (1) visuelles, wenig fokussiertes Anschauen, z.B. der Blick auf eine Regalfläche oder einen Auslegetisch (2) visuelle, fokussierte Zuwendung, z.B. Anschauen einer bestimmten Ware oder Artikelgruppe, (3) Anfassen und Zurücklegen eines Artikels und (4) Anfassen, Anschauen und Mitnahme eines Artikels. 
Dieses Zuwendungsverhalten lässt sich per Ortungssysteme nicht automatisch registrieren. Es dürfte außerdem schwierig sein, mit installierten Raumkameras fokussiertes Schauen oder das Anfassen von Waren hinter einem Regal zu erfassen. Die im Labor hinter dem Regal einsetzten Kameras, ggf. hinter einem Einwegspiegel, wären sicherlich viel zu aufwendig und setzen zudem halbleere Regale voraus. Deshalb ist vor allem an die klassische Beobachtung vor Ort zu denken. Dasselbe gilt für die parallele Auskunftserteilung bei der Handle-LautMethode. Eine Nachherbefragung muss gerade beim Zuwendungsverhalten mit großen Erinnerungslücken rechnen, da ein Ladenbesucher i.d.R. andere Dinge zu tun hat, als sich selbst in allen Details, auch in seinem Schauen, selbst zu beobachten.

Besondere Bedeutung kommt dem Kaufverhalten zu, entscheidet es doch nicht nur über den Kauferfolg des Nachfragers, sondern auch über den Markterfolg der Anbieter - den Erfolg des Händlers, aber auch den Erfolg der Hersteller jener Waren, die im Geschäft angeboten werden. Das Kaufverhalten ist im stationären Handel als Mitnahme von Ware gegen Entgelt zu verstehen, das Mitnehmen bezahlter Ware beim Verlassen des Geschäfts. Daraus folgt, dass die Entnahme von Ware am Regal streng genommen keinen Kauf darstellt, weil die Ware an derselben oder an anderer Stelle wieder abgelegt, verzehrt oder entwendet werden kann. Solche Sachverhalte lassen sich von aufmerksamen und geschulten Beobachtern zumindest größtenteils erfassen. Was an der Kasse bezahlt wird, registriert die Scannerkasse. Doch ist damit noch keine Zuordnung zu einer bestimmten Person gesichert. Ausnahmen sind Kassenvorgänge mit Kundenkarte oder hauseigenen Kreditkarten, das Notieren von Personenangaben durch das Kassenpersonal und Beobachter, die Kassenzettel und Personenangaben per Hand registrieren. Denkbar sind auch Registriersysteme in Einkaufswagen und Einkaufskörben, die z.B. RFID-gestützt festhalten, was wann und wo eingelegt und herausgenommen wurde. Aber auch hier ist eine Personenzuordnung nicht ohne weiteres möglich. 
Das im Ladengeschäft erfasste Zuwendungsverhalten, die Regalentnahme von Ware eingeschlossen, kann als Sequenz bestimmt und ausgewertet werden. Dies ist jedoch nicht so zwingend, wie es beim Laufverhalten der Fall ist. Deshalb kann es genügen, wenn lediglich der Ort und die Häufigkeit von Zuwendungsreaktionen festgehalten und ausgewertet werden. Es macht jedenfalls Sinn, Zuwendungsreaktionen auf den Adressaten der Zuwendung zu beziehen, z.B. auf ein Regal, eine Warengruppe, einen Artikel, einen Service Point, einen Mitarbeiter oder ein Innenplakat. Jedenfalls lassen sich Zuwendungen auch den Stationen eines Kundenlaufs zuordnen, wenn pro Besucher nicht nur dessen Zuwendungsverhalten, sondern auch dessen Laufverhalten erfasst wird.

\section{(3) Die kognitiven Prozesse und deren Erfassung}

Bezogen auf das innere Verhalten am POS liegt es nahe, in erster Linie an die Kaufüberlegungen zu denken, die einem Kauf- oder Mitnahmeakt vorangehen. Darüber hinaus sollte interessieren, ob Kaufabsichten vergessen und ob Kaufpläne aufgegeben werden. Dasselbe gilt für Reaktionen vor dem leeren Regal. Das sog. Einkaufserleben umfasst darüber hinaus auch die Wahrnehmung derjenigen Angebote, die nicht gekauft werden wollen, die Wahrnehmung der Ladengestaltung, der Gestaltung von Auslagen, der Kommunikationsmaßnahmen, schließlich auch die Wahrnehmung des Verhaltens der Mitarbeiter und anderer Ladenbesucher und der Besucher, die vor Fremdbedienungstheken und vor Kassenterminals anstehen. Ein inneres Verhalten muss aber keineswegs immer nur Auslöser und Adressaten aus der Umwelt haben. Gedanken können sich auch ausschließlich auf die eigene Personen beziehen, so z.B. auf die Empfindungen und auf Pläne, Erwartungen, Wünsche und Befürchtungen, die aktuell verfügbare Kaufkraft nicht zu vergessen.

Für die Erfassung des inneren Verhaltens kommt nur die Befragungsmethode

in Betracht. Handelt es sich um Dinge, die kaum bewusst und solche, die leicht vergessen werden, muss die ungestützte Erinnerung im Rahmen einer 
Nachherbefragung versagen. Dann bleiben nur noch die Nachherbefragung mit Erinnerungsstützen und die sog. Denke-Laut-Methode. Und soll der Einfluss des lauten Denkens auf das Einkaufserleben ausgeschlossen werden, bleibt letztlich nur noch das Nachherbefragen mit Erinnerungsstützen - die gestützte Rekonstruktion der Gedanken.

Bei der Auswertung von kognitiven Prozessen kommt das Problem einer Codierung von Sachverhalten hinzu. Klassifizierungen von Gedanken setzen meist nicht nur bewährte Raster voraus, sondern auch Vorstudien, die die Eignung vorgefertigter Raster überprüfen und die Gelegenheit zur Anpassung dieser Raster eröffnen. Wie beim Zuwendungsverhalten kann neben den Häufigkeiten bestimmter Gedanken auch deren Abfolge interessieren, muss aber nicht. Es ist auf jeden Fall von Vorteil, wenn Teile des inneren Verhaltens den Stationen im Kundenlauf und den relevanten Zuwendungsaktivitäten zugeordnet werden können. Dies ist weniger eine Herausforderung an die Datenauswertung, als vielmehr eine Herausforderung an die Datenerhebung.

\section{Ein Drei-Stufen-Modell für die Verhaltensforschung am POS}

Nachdem die verschiedenen Ebenen des Verhaltens beim Ladenbesuch sowie entsprechende Erfassungsmöglichkeiten dargestellt worden sind, sei nun ein Vorschlag bezüglich der künftigen Verhaltensforschung im Ladengeschäft zu unterbreitet. Die Entwicklung dieses Vorschlages geht von folgenden Grundannahmen aus:

(1) Das Verhalten der Ladenbesucher lässt sich in drei Bereiche einteilen: das Laufverhalten, das Zuwendungs- sowie Kaufverhalten und die kognitiven Prozesse. (2) Das innere Verhalten erfordert den Einsatz der Befragungs- und Rekonstruktionsmethoden, das Zuwendungsverhalten legt den Einsatz der Beobachtungsmethode nahe und das Laufverhalten kann automatisch registriert werden. 
(3) Die Akzeptanz bzw. die Teilnahmebereitschaft der Besucher ist bei der automatischen, anonymen Registrierung von Laufwegen größer als bei der Beobachtung des Zuwendungsverhaltens, und bei der Beobachtung des Zuwendungsverhaltens größer als bei der Abfrage und Rekonstruktion kognitiver Prozesse.

(4) Auch ohne die gleichzeitige Erfassung aller Verhaltensdimensionen in einund derselben Stichprobe sind Einsichten in die Interdependenz der verschiedenen Verhaltensdimensionen möglich.

(5) Eine abgestufte Verhaltensforschung kann helfen, den Aufwand bei der Erkundung verschiedener Verhaltensdimensionen zu verringern.

Der im Folgenden präsentierte Vorschlag empfiehlt ein dreistufiges Vorgehen. Die Forschung beginnt mit der automatischen, anonymen und wenig aufwendigen Registrierung des Laufverhaltens aller Ladenbesucher. Sie fährt mit der verdeckten Beobachtung des Zuwendungs- und Kaufverhaltens ausgewählter Ladenbesucher fort und endet schließlich mit der Abfrage oder Rekonstruktion der kognitiven Prozesse. Die erste Stufe soll mit geringem Aufwand für flächendeckende Hinweise auf Laufmuster sorgen, die zweite Stufe mit gesteigertem, aber begrenztem Aufwand für stichprobenartige Befunde zum Zuwendungs- und Kaufverhalten und die dritte Stufe mit noch mehr Aufwand, aber kleineren Stichproben für die Analyse der kognitiven Prozesse.

\subsection{Erste Stufe: Registrierung des Kundenlaufs}

Als erste Stufe schlagen wir eine automatische Ortung als anonymes Tracking aller Besucher in ihrem Laufverhalten vor. Diese Vollerhebung schließt ein „time sampling“ nicht aus, bei dem die Erfassung - auch im Interesse der Kunden - auf bestimmte Zeiträume begrenzt wird. Das Registriersystem könnte so ausgelegt werden, dass alle Lauf- und Aufenthaltsflächen abgedeckt sind. Die technische Realisierbarkeit und die systembezogene generelle Akzeptanz der Ladenbesucher sprechen am ehesten für eine Ortung der Einkaufswagen und der Einkaufskörbe. 
Dabei ist allerdings zu konzedieren, dass die Bewegung der Einkaufswagen und der Einkaufskörbe nicht mehr sein kann als eine Annäherung an das Laufverhalten der jeweiligen Ladenbesucher. Außerdem muss damit gerechnet werden, dass keineswegs alle Ladenbesucher einen Einkaufswagen oder einen Einkaufskorb des jeweiligen Ladengeschäfts benutzen. Deswegen kann - streng genommen - auch nicht von einer Vollerhebung gesprochen werden.

Die Auswertung der registrierten Bewegungen bei der Ermittlung von Laufmustern kann ebenfalls automatisch erfolgen. Der Vergleich der Laufwegsdaten im Rahmen der Sequenzanalyse und deren Clusterung liegt auf der Hand. Bei der vorprogrammierten Datenauswertung lassen sich auch bestimmte Kennwerte berechnen und in die Clusterbeschreibung einbeziehen, so z.B. die Anzahl der betretenen Lauf- und Präsentationsflächen, die Eintritts- und Austrittszeit, aus denen sich die Besuchsdauer errechnen lässt, und die grob geschätzte, zurückgelegte Wegstrecke, so dass sich aus der geschätzten Wegstrecke und der erfassten Besuchsdauer auch eine grobe Schätzung der durchschnittlichen Laufgeschwindigkeit errechnen lässt.

Auf welche Weise die so ermittelten Laufcluster beschrieben werden können, sei nun anhand eines Untersuchungsbeispiels demonstriert. In einer Studie, die in der Weihnachtsabteilung eines Verbrauchermarktes (unter der Mitarbeit von Manuel Rauch) durchgeführt wurde (siehe dazu den Grundriss mit den 16 Laufflächen und den 30 Präsentationsflächen in Abbildung 1), ergaben sich per Beobachtung des Kundenlaufs ( $\mathrm{n}=188)$ und als Ergebnis der Clusterung von Laufflächensequenzen drei unterschiedliche Laufmuster. Eine Darstellung der Zentroide und einiger

Laufwege, die dem jeweiligen Zentroid am nächsten kommen, den sog. Zentroid-Proxies, findet sich in Tabelle 1. Diese Darstellung lässt nicht nur das Zentrum des Laufmusters, sondern auch die unterschiedliche Länge der jeweiligen Laufwege erkennen. 


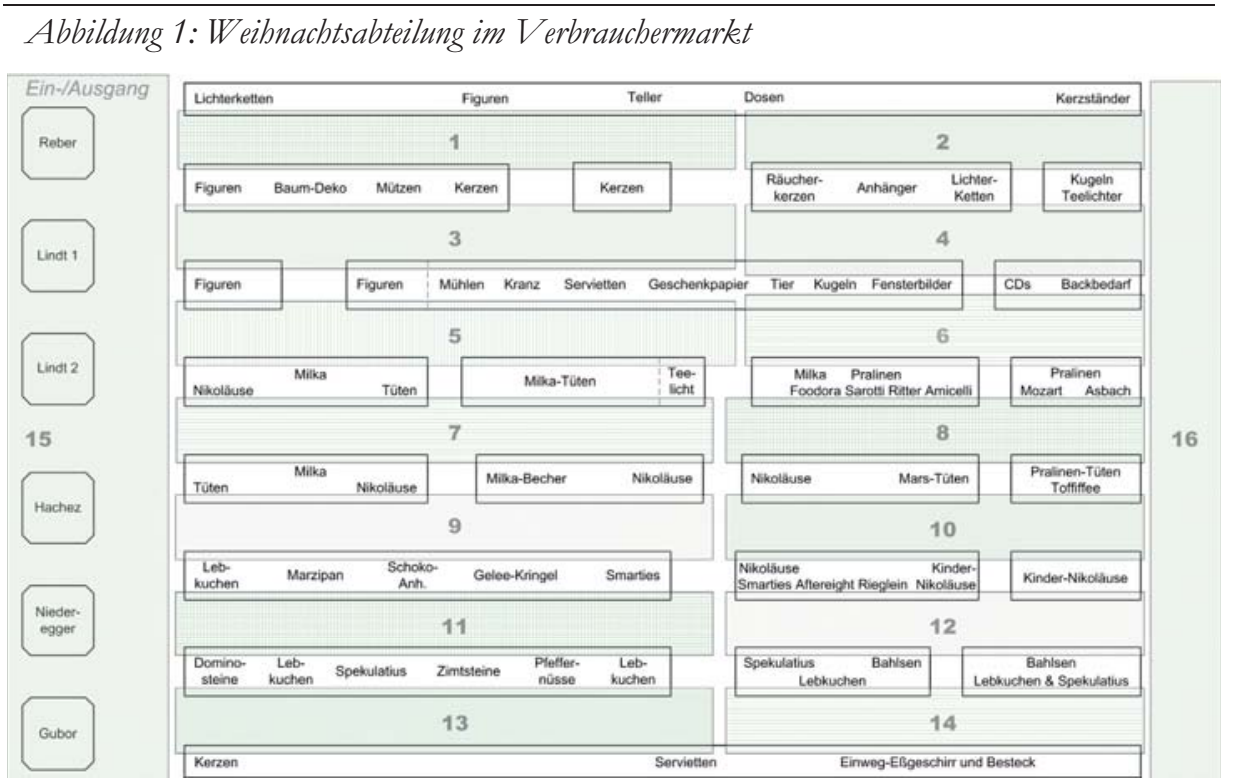

Tabelle 1: Laufcluster in der Weibnachtsabteilung. Beschreibung anhand der Zentroide und Zentroid-Proxies

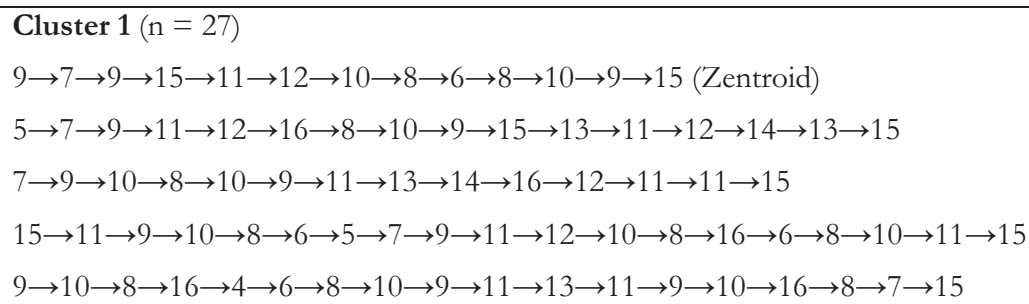

Cluster $2(\mathrm{n}=88)$

$7 \rightarrow 8 \rightarrow 10 \rightarrow 9 \rightarrow 11 \rightarrow 11 \rightarrow 15$ (Zentroid)

$9 \rightarrow 7 \rightarrow 9 \rightarrow 15$

$11 \rightarrow 12 \rightarrow 11 \rightarrow 15$

$9 \rightarrow 7 \rightarrow 10 \rightarrow 9$

$9 \rightarrow 9 \rightarrow 12 \rightarrow 11 \rightarrow 15$

\section{Cluster $3(\mathrm{n}=73)$}

$$
\begin{aligned}
& 1 \rightarrow 2 \rightarrow 4 \rightarrow 3 \rightarrow 15 \rightarrow 5 \rightarrow 7 \rightarrow 15 \text { (Zentroid) } \\
& 1 \rightarrow 2 \rightarrow 2 \rightarrow 4 \rightarrow 16 \rightarrow 6 \rightarrow 5 \rightarrow 15 \\
& 1 \rightarrow 3 \rightarrow 3 \rightarrow 4 \rightarrow 6 \rightarrow 5 \rightarrow 15 \\
& 3 \rightarrow 4 \rightarrow 2 \rightarrow 4 \rightarrow 2 \rightarrow 1 \rightarrow 1 \rightarrow 15 \\
& 1 \rightarrow 3 \rightarrow 5 \rightarrow 3 \rightarrow 1 \rightarrow 15
\end{aligned}
$$

Clustermethode: Ward's method, Dissimilarities from Levenshtein`s distance matrix. 
Tabelle 2: Beschreibung der Laufcluster anhand der Häufigkeit der betretenen Laufflächen

\begin{tabular}{|c|c|c|c|c|c|c|}
\hline & \multirow{2}{*}{$\begin{array}{c}\text { Cluster } 1 \\
(\mathrm{n}=27) \\
\varnothing\end{array}$} & \multirow{2}{*}{$\begin{array}{c}\text { Cluster } 2 \\
(\mathrm{n}=88) \\
\varnothing\end{array}$} & \multirow{2}{*}{$\begin{array}{c}\text { Cluster } 3 \\
(\mathrm{n}=73) \\
\varnothing\end{array}$} & \multicolumn{3}{|c|}{$\begin{array}{c}\text { Signifikanz der } \\
\text { Differenz }\end{array}$} \\
\hline & & & & $1-2$ & $1-3$ & $2-3$ \\
\hline $\begin{array}{l}\text { Lauffläche Nr. } 1 \\
\end{array}$ & $0,11(0,42)$ & $0,01(0,11)$ & $0,71(0,79)$ & n.s. & *** & *** \\
\hline Lauffläche Nr. 2 & $0,19(0,68)$ & $0,01(0,11)$ & $0,74(0,94)$ & n. s. & $* *$ & $* * *$ \\
\hline Lauffläche Nr. 3 & $0,15(0,46)$ & $0,01(0,11)$ & $0,86(1,03)$ & n. s. & $* * *$ & $* * *$ \\
\hline Lauffläche Nr. 4 & $0,33(0,96)$ & $0,00(0,00)$ & $0,89(1,01)$ & $\mathrm{T}$ & $*$ & $* * *$ \\
\hline Lauffläche Nr. 5 & $0,85(0,86)$ & $0,30(0,55)$ & $0,70(0,68)$ & ** & n. s. & $* * *$ \\
\hline Lauffläche Nr. 6 & $1,22(1,25)$ & $0,19(0,43)$ & $0,60(0,57)$ & $* * *$ & * & $* * *$ \\
\hline Lauffläche Nr. 7 & $1,30(0,91)$ & $0,63(0,73)$ & $0,25(0,55)$ & $* * *$ & $* * *$ & $* * *$ \\
\hline Lauffläche Nr. 8 & $2,11(1,15)$ & $0,52(0,62)$ & $0,45(0,69)$ & $* * *$ & $* * *$ & n. s. \\
\hline Lauffläche Nr. 9 & $2,44(1,22)$ & $1,20(0,82)$ & $0,40(0,62)$ & $* * *$ & $* * *$ & $* * *$ \\
\hline Lauffläche Nr. 10 & $2,37(1,21)$ & $0,78(0,63)$ & $0,45(0,62)$ & $* * *$ & $* * *$ & $* * *$ \\
\hline Lauffläche Nr. 11 & $1,59(0,69)$ & $0,80(0,78)$ & $0,55(0,80)$ & $* * *$ & $* * *$ & $*$ \\
\hline Lauffläche Nr. 12 & $1,44(0,75)$ & $0,42(0,58)$ & $0,34(0,53)$ & $* * *$ & $* * *$ & n. s. \\
\hline Lauffläche Nr. 13 & $1,04(0,90)$ & $0,35(0,57)$ & $0,36(0,67)$ & $* * *$ & $* * *$ & n. s. \\
\hline Lauffläche Nr. 14 & $0,78(0,70)$ & $0,17(0,41)$ & $0,19(0,46)$ & $* * *$ & $* * *$ & n. s. \\
\hline Lauffläche Nr. 15 & $2,81(1,00)$ & $2,22(0,73)$ & $2,23(0,95)$ & ** & $* *$ & n. s. \\
\hline Lauffläche Nr. 16 & $1,11(0,85)$ & $0,38(0,55)$ & $0,67(0,78)$ & $* * *$ & $* *$ & $* *$ \\
\hline $\begin{array}{l}\text { Flächenkontakte } \\
\text { insges. }\end{array}$ & $21,33(5,94)$ & $8,92(2,76)$ & $11,32(3,65)$ & $* * *$ & *** & $* * *$ \\
\hline
\end{tabular}

***/**/*/T $=1 \% 0 / 1 \% / 5 \% / 10 \%$-Niveau, n. s. $=$ nicht signifikant, t-Test, 2seitig. sd in Klammern.

Eine weitere Beschreibung der Laufmuster findet sich in Tabelle 2, die über die jeweilige Häufigkeit der Laufflächenkontakte berichtet. Tabelle 3 zeigt dann die Unterschiede bezüglich sonstiger Variablen, die bei der automatischen Registrierung ebenfalls anfallen, nämlich bezüglich der geschätzten Länge des Laufwegs und der errechneten Besuchsdauer, weniger dagegen bezüglich der Eintrittszeit und der errechneten Geschwindigkeit (in Metern pro Minute).

Verglichen mit etwas einfacher zu bestimmenden Besuchsfrequenzen in den Abteilungen oder Arealen eines Ladengeschäfts bieten die Befunde zum Laufverhalten den Vorteil, dass sie nicht nur die Abfolge der begangenen Flächen anzeigen, sondern auch den ggf. erfolgten Mehrfachbesuch einer Fläche, den 
benutzen Eingang, die benutzte Kassenzone und den benutzten Ausgang, auch die Aufenthaltsdauer und die grob geschätzte Laufgeschwindigkeit.

Tabelle 3: Beschreibung der Laufcluster anhand zeit- und laufwegbezogener Merkmale

\begin{tabular}{|c|c|c|c|c|c|c|}
\hline & $\begin{array}{l}\text { Cluster } 1 \\
(\mathrm{n}=27)\end{array}$ & $\begin{array}{l}\text { Cluster } 2 \\
(\mathrm{n}=88)\end{array}$ & $\begin{array}{l}\text { Cluster } 3 \\
(\mathrm{n}=73)\end{array}$ & \multicolumn{3}{|c|}{$\begin{array}{l}\text { Signifikanz } \\
\text { der } \\
\text { Differenz }\end{array}$} \\
\hline & $\varnothing(\mathrm{sd})$ & $\varnothing(\mathrm{sd})$ & $\varnothing(\mathrm{sd})$ & $1-2$ & -3 & $2-3$ \\
\hline $\begin{array}{l}\text { Eintritt in die Abteilung, } \\
\text { Std.:Min. }\end{array}$ & 15:01 (2:42) & $14: 28(2: 40)$ & $13: 58(2: 36)$ & n. s. & $\mathrm{T}$ & n. s. \\
\hline $\begin{array}{l}\text { Geschätzter Laufweg, } \\
\text { in Metern }\end{array}$ & $116,61(47,92)$ & $56,73(19,93)$ & $71,55(27,23)$ & $* * *$ & $* * *$ & $* * *$ \\
\hline $\begin{array}{l}\text { Besuchsdauer, } \\
\text { Min.:Sek. }\end{array}$ & 8:01 (5:21) & $3: 42(2: 10)$ & $4: 22(2: 18)$ & $* * *$ & $* * *$ & $\mathrm{~T}$ \\
\hline $\begin{array}{l}\text { Geschätzte } \\
\text { Geschwindigkeit, } \mathrm{m} / \mathrm{min}\end{array}$ & $18,90(11,43)$ & $19,77(13,03)$ & $21,41(15,48)$ & n. s. & n. s. & n. s. \\
\hline
\end{tabular}

\subsection{Zweite Stufe: Beobachtung des Zuwendungs- und Kaufverhaltens}

Für die zweite Stufe schlagen wir eine Beobachtung von ausgewählten Ladenbesuchern in ihrem Zunvendungs- und Kaufverhalten vor. Dieses Verhalten kann in der ersten Stufe nicht erfasst werden. Und da die Beobachtung viel aufwendiger ist als die Registrierung, sollte und kann sie sich auf eine oder mehrere gezielte Stichproben beschränken.

Um die Selektivität der entsprechenden Stichprobe(n) so klein wie möglich zu halten, empfiehlt sich eine verdeckte Beobachtung, deren Machbarkeit in verschiedenen Studien gezeigt werden konnte. Sie setzt allerdings geeignete und geschulte Beobachter voraus, da sie nicht als solche erkannt werden sollen - weder von den beobachteten Personen, noch von anderen Ladenbesuchern. Sie registrieren Zuwendungen zum Angebot wie z.B. Anschauen, Anfassen, Zurücklegen und 
Mitnehmen, Interaktionen mit der Kaufbegleitung, Interaktionen mit dem Verkaufs- und Servicepersonal, die Rückgabe von Leergut, das Konsumverhalten in Gastronomiebereichen, die Anzahl der Artikel, die an der Kassenzone bezahlt und somit auch wirklich gekauft werden, ggf. auch die Art des Warenabtransports.

Da in der zweiten Stufe ebenfalls Sequenzdaten und Feststellungen zur Besuchdauer in Verkaufs- und Rekreationszonen anfallen, kommen wiederum die Sequenzanalyse und der Vergleich von Sequenzen, ggf. auch deren Clusterung, in Betracht. Um eine erste Erklärung des Zuwendungs- und Kaufverhaltens zu ermöglichen, sollten im Rahmen der Beobachtung auch personenbezogene Merkmale festgehalten werden, so z.B. die Benutzung eines Einkaufswagens oder eines Einkaufskorbes, die Anzahl der Begleitpersonen, die Fülle des Wagen bzw. des Korbes, die taxierte Eile, die sich im Verhalten ausdrückt, das geschätzte Alter und das Geschlecht der beobachteten Personen.

In der zweiten Stufe lässt sich ohne nennenswerten Zusatzaufwand auch das Laufverhalten feststellen. Haben die Beobachter auch dieses Verhalten festgehalten, könnte es gelingen, die Befunde der zweiten Stufe den Laufmustern oder Laufclustern aus der ersten Stufe zuzuordnen. Dieser Vorteil ist nicht zu unterschätzen, selbst dann, wenn die Stichprobe(n) aus der zweiten Stufe zu klein ist (sind), um eine Zuordnung des Zuwendungs- und Kaufverhaltens zu allen Laufmustern aus Stufe 1 vornehmen zu können. Dieser Nachteil lässt jedoch begrenzen, wenn die Stichprobengröße in Stufe 2 vorerst offen bleibt und an die Ergebnisse bzw. an das Erfordernis einer Zuordnung von Lauf- und Zuwendungsverhalten (just-in-time) angepasst wird. Dasselbe gilt, wenn das Zuwendungsverhalten nur für ganz bestimmte Laufcluster bestimmt werden soll. Werden die Ergebnisse der Kundenlaufbestimmung aus Stufe 1 mit den Ergebnissen der Kundenlaufbestimmung aus Stufe 2 verglichen, eröffnet dies im Übrigen auch die Möglichkeit, die Selektivität der Beobachtung in Stufe 2 zu bestimmen. 
Mögliche Ergebnisse einer verdeckten Beobachtung des Zuwendungsverhaltens und anderer beobachtbarer Sachverhalte sollen wiederum am Beispiel einer konkreten Studie demonstriert werden. Es handelt sich wiederum um die oben skizzierte Studie zum Besucherverhalten in der Weihnachtsabteilung eines Verbrauchermarktes. Die in Tabelle 4 dargestellten Ergebnisse ergänzen - wie erwartet - die Resultate der Laufregistrierung. Dabei zeigt sich, dass die Unterschiede zwischen den Laufclustern vor allem das Zuwendungsverhalten betreffen, weniger die Personen- bzw. Gruppenmerkmale.

In der zweiten Stufe wird aus mehreren Gründen auf eine Nachbefragung der verdeckt beobachteten Ladenbesucher verzichtet. Zum einen würde die Nachbefragung beachtlichen Aufwand verursachen. Zudem müsste damit gerechnet werden, dass nicht alle Besucher einer solchen Befragung zustimmen können oder wollen. Und eine Vorabfrage der Interviewbereitschaft könnte durchaus nennenswerte Reaktivität der Probanden während des Ladenbesuchs erzeugen. Außerdem müssten die Befragten über die erfolgte Beobachtung aufgeklärt werden, wenn geplant ist, die Befragungsdaten und die Beobachtungsdaten später zu fusionieren. Und diese Aufklärung könnte nicht zuletzt bewirken, dass über die Mund-zu-Mund-Kommunikation zwischen Ladenbesuchern innerhalb und außerhalb des Geschäfts die Beobachtung des öfteren als solche erkannt wird. 
Tabelle 4: Beschreibung der Laufcluster anhand beobachteter Zuwendungen und anderer Sachverhalte

\begin{tabular}{|c|c|c|c|c|c|}
\hline & $\begin{array}{l}\text { Cluster } 1 \\
(\mathrm{n}=27)\end{array}$ & $\begin{array}{l}\text { Cluster } 2 \\
(\mathrm{n}=88)\end{array}$ & $\begin{array}{l}\text { Cluster } 3 \\
(\mathrm{n}=73)\end{array}$ & & $\begin{array}{l}\text { Signifikanz } \\
\text { der } \\
\text { Differenz }\end{array}$ \\
\hline & $\begin{array}{c}\varnothing \text { (Anteil } \\
\text { in } \%)\end{array}$ & $\begin{array}{c}\varnothing \text { (Anteil } \\
\text { in } \%)\end{array}$ & $\begin{array}{c}\varnothing \text { (Anteil } \\
\text { in } \%)\end{array}$ & $1-2$ & -3 \\
\hline Anzahl laufender Personen & $1,33(0,48)$ & $1,46(0,64)$ & $1,1,47(0,71)$ & n. s. & n. s. n.s. \\
\hline $\begin{array}{l}\text { Geschlecht } \\
\text { (Anteil Frauen), in \% }\end{array}$ & $81 \%$ & $78 \%$ & $86 \%$ & n. s. & n. s. n. s. \\
\hline Personen mit Wagen/Korb, in $\%$ & $78 \%$ & $76 \%$ & $72 \%$ & n. s. & n. s. n. s. \\
\hline Fülle des Wagens/Korbs ${ }^{1}$ & $2,10(1,18)$ & $1,80(1,18)$ & $2,04(1,12)$ & n. s. & n. s. $\mathrm{T}$ \\
\hline Geschätzte Eile ${ }^{2}$ & $0,52(0,85)$ & $0,59(0,84)$ & $0,53(0,79)$ & n. s. & n. s. n. s. \\
\hline Geschätzte Altersklasse ${ }^{3}$ & $2,11(0,70)$ & $2,15(0,67)$ & $2,01(0,72)$ & n. s. & n.s. n.s. \\
\hline $\begin{array}{l}\text { Geschätztes Outfit } \\
\left(3 \text { Stufen }{ }^{4}\right)\end{array}$ & $1,89(0,85)$ & $1,86(0,84)$ & $1,82(0,84)$ & n. s. & n. s. n. s. \\
\hline
\end{tabular}

Häufigkeit der Zuwendungen:

- Produktgruppe betrachtet $0,04(0,19) \quad 0,20(0,66) \quad 0,22(0,61) \quad * * * \quad * * * \quad$ n. s.

- bestimmtes Produkt betrachtet

$2,04(1,51)$

$1,14(1,38)$

$1,51(1,37)$

* $\quad *$ n. s.

- Produkt herausgenommen

$2,93(3,32)$

$1,00(1,22)$

$1,59(1,57)$

** T

- Produkt mitgenommen

$2,70(2,15)$

$1,67(1,62)$

$1,19(1,44)$

- Zuwendungshäufigkeit insges.

$7,70(3,62)$

$4,01(2,23)$

$4,51(2,36)$

$* * * / * * / * / \mathrm{T}=1 \% 0 / 1 \% / 5 \% / 10 \%$-Niveau, n. $\mathrm{s} .=$ nicht signifikant, t-Test, 2seitig. sd in Klammern.

${ }^{1}$ Skala: „, "“ - leer, ,2“ - wenig o. Korb, ,3“ - viel drin.

${ }^{2}$ Skala: „, 0 “ - nein, „1“- unklar, „2“ - ja.

${ }^{3}$ Skala: , ,1“ = bis unter $25 \mathrm{~J} .$, , ,2“ $=25 \mathrm{~J}$. bis unter $45 \mathrm{~J} .,, 3^{\prime \prime}=45 \mathrm{~J}$ und älter

${ }^{4}$ Skala: , $1^{\prime \prime}=$ einfach, , $2^{\prime \prime}=$ weder noch, , $3 “=$ hochwertig

\subsection{Dritte Stufe: Abfrage und Rekonstruktion kognitiver Prozesse}

Die dritte Stufe dient der Erfassung kognitiver Prozesse während des Ladenbesuchs. Die Erfassung dieser Prozesse ist nicht nur aufwendig, sondern muss auch mit Selektivitäts- und Reaktivitätseffekten rechnen. Dies trifft in einem besonderen Masse bei der Denke-Laut-Methode zu. Deshalb sind die 
Nachherbefragung und die Rekonstruktion kognitiver Prozesse nach dem Besuch der Denke-Laut-Methode vorzuziehen.

Bezüglich einer Nachherbefragung sind zwei Fälle zu unterscheiden. Entweder handelt es sich um kleine Geschäfte bzw. um solche, in denen der kurze Besuch eindeutig dominiert, so dass eine Nachherbefragung ohne Gedächtnisstütze durchaus in Frage kommt, oder es handelt sich um große Geschäfte bzw. um solche, in denen auch mit einer längeren Besuchsdauer gerechnet werden muss, so dass eine Rekonstruktion kognitiver Prozesse nur mit Erinnerungsstütze möglich ist. Sollen in einem größeren Geschäft nur jene kognitiven Prozesse untersucht werden, die beim Besuch einer bestimmten Abteilung oder eines bestimmten kleineren Areals ablaufen, dann kommt auch die ungestützte Gedankenabfrage kurz nach dem Besuch der betreffenden Fläche in Frage.

Für jene Fälle, in denen eine gestützte Rekonstruktion kognitiver Prozesse erforderlich ist, liegt folgendes Vorgehen nahe: Die Beobachtung oder Aufnahme des Lauf-, Zuwendungs- und Kaufverhaltens, um das Resultat dieser Beobachtung bzw. dieser Aufnahme als Gedächtnisstütze einsetzen zu können. Hierbei kommen mehrere Möglichkeiten in Frage: die Skizze und der verbale Bericht einer Begleitperson, Videoaufnahmen eines mitlaufenden Kameramannes und Aufnahmen einer Blickfeldkamera. Dabei handelt es sich um Erinnerungshilfen, die das Verhalten und die jeweilige Verhaltenssituation zumindest partiell anschaulich und in der richtigen Reihenfolge wiedergeben. Dieser Vorteil muss mit dem Nachteil erkauft werden, dass der Beobachtungsvorgang bzw. die Videoaufnahmen in aller Regel nicht verdeckt erfolgen können, sondern offen gelegt sein müssen, was eine Bitte um Erlaubnis vor dem Beobachtungs- bzw. Aufnahmevorgang erfordert und die Erlaubnis des Probanden voraussetzt.

Kommt es in Stufe 3 aus den genannten Gründen zu einer Erfassung des Lauf, Zuwendungs- und Kaufverhaltens, so ergeben sich daraus zwei nützliche Nebeneffekte: Zum einen können aus dem Vergleich der Befunde in Stufe 3 mit den Ergebnissen der verdeckten Beobachtung in Stufe 2 erste Anhaltspunkte 
bezüglich möglicher Reaktivitätseffekte beim Lauf-, Zuwendungs- und Kaufverhalten einer offenen Beobachtung gewonnen werden, zum anderen lassen sich Hinweise auf die Selektivität der Stichprobe in Stufe 3 anhand eines Vergleichs der Befunde zum Kundenlauf in Stufe 1 und in Stufe 3 gewinnen.

Sowohl bei der einfachen Nachbefragung als auch im Rahmen einer gestützten Rekonstruktion der Gedanken besteht immer auch die Möglichkeit, mögliche Motive oder Ursachen des Lauf-, Zuwendungs- und Kaufverhaltens zu erfragen und selbst Fragen zur Wahl des jeweiligen Geschäfts zu stellen. Anhand der oben bereits skizzierten Studie zum Besucherverhalten in einer Weihnachtsabteilung können auch diesbezüglich einige Möglichkeiten aufgezeigt werden. Als Personenmerkmale interessierten das genaue Alter sowie die Stimmung beim Betreten und beim Verlassen der Abteilung. Außerdem wurde gefragt, ob die gewünschten Produkte gefunden und ob auch ungeplante Käufe getätigt wurden. Darüber hinaus sollten die Probanden berichten, welche Gedanken ihnen in dieser Abteilung durch den Kopf gingen. Schließlich wurden die Befragten gebeten, Angaben zu ihrer Kaufzufriedenheit, zur Beurteilung der Abteilung und der geforderten Preise zu machen sowie zu sagen, ob sie diese Abteilung vor Weihnachten noch einmal besuchen wollen. 
Tabelle 5: Beschreibung der Laufcluster anhand von Ergebnissen der Nachbefragung

\begin{tabular}{|c|c|c|c|c|c|c|}
\hline & Cluster 1 & Cluster 2 & Cluster 3 & \multirow{2}{*}{\multicolumn{3}{|c|}{$\begin{array}{l}\text { Signifikanz } \\
\text { der } \\
\text { Differenz }\end{array}$}} \\
\hline & $(\mathrm{n}=23)$ & $(\mathrm{n}=69)$ & \multirow{2}{*}{$\begin{array}{l}(\mathrm{n}=66) \\
\varnothing(\mathrm{sd})\end{array}$} & & & \\
\hline & $\varnothing(\mathrm{sd})$ & $\varnothing(\mathrm{sd})$ & & $1-2$ & $1-3$ & $2-3$ \\
\hline Genaues Alter & $\begin{array}{c}39,83 \\
(15,90)\end{array}$ & $\begin{array}{l}39,62 \\
(14,10)\end{array}$ & $\begin{array}{l}37,88 \\
(12,41)\end{array}$ & n. s. & n. s. & n. s. \\
\hline Stimmung beim Eintritt ${ }^{1}$ & $2,13(0,82)$ & $2,28(0,71)$ & $2,33(0,59)$ & n. s. & n. s. & n. s. \\
\hline Stimmung beim Austritt ${ }^{1}$ & $2,22(0,95)$ & $2,28(0,77)$ & $2,53(0,73)$ & n. s. & n. s. & * \\
\hline Stimmungsdifferenz & $-0,09(0,60)$ & $0,00(0,42)$ & $-0,20(0,64)$ & n. s. & n. s. & * \\
\hline $\begin{array}{l}\text { Anteil der Personen, die gewünschte } \\
\text { Produkte gefunden, in \% }\end{array}$ & $95 \%$ & $88 \%$ & $62 \%$ & n. s. & $\mathrm{T}$ & $*$ \\
\hline $\begin{array}{l}\text { Anteil der Personen, die gewünschte } \\
\text { Produkte gekauft, in \% }\end{array}$ & $94 \%$ & $82 \%$ & $59 \%$ & n. s. & $*$ & $\mathrm{~T}$ \\
\hline $\begin{array}{l}\text { Anzahl der Personen mit ungeplanten } \\
\text { Käufen }\end{array}$ & $0,87(2,14)$ & $0,65(1,07)$ & $0,75(1,06)$ & n. s. & n. s. & n. s. \\
\hline Zufriedenheit mit dem Kauf ${ }^{1}$ & $4,09(0,42)$ & $3,87(0,59)$ & $3,68(0,61)$ & n. s. & n. s. & n. s. \\
\hline Bewertung der Abteilung $^{1}$ & $3,17(0,83)$ & $3,57(0,87)$ & $3,55(0,90)$ & $\mathrm{T}$ & $\mathrm{T}$ & n. s. \\
\hline $\begin{array}{l}\text { Einschätzung der Preise in der } \\
\text { Abteilung }^{1}\end{array}$ & $3,48(1,08)$ & $3,10(0,81)$ & $3,26(0,81)$ & $\mathrm{T}$ & n. s. & n. s. \\
\hline Wiederkehrabsicht $^{2}$ & $2,22(0,90)$ & $2,61(0,69)$ & $2,62(0,78)$ & $\mathrm{T}$ & $\mathrm{T}$ & n. s. \\
\hline Häufigkeit der Kognitionen: & & & & & & \\
\hline - Intentionen (IZ) & $1,13(1,06)$ & $0,84(0,66)$ & $1,20(0,77)$ & n. s. & n. s. & $* *$ \\
\hline - Alternativen-Wahrnehmung (AW) & $0,48(0,51)$ & $0,51(0,58)$ & $0,29(0,46)$ & n. s. & n. s. & * \\
\hline - Alternativenbewertung (AB) & $0,43(0,59)$ & $0,35(0,61)$ & $0,43(0,61)$ & n. s. & n. s. & n. s. \\
\hline - Orientierungsschwierigkeiten $(\mathrm{ON})$ & $0,35(0,57)$ & $0,36(0,62)$ & $0,46(0,61)$ & n. s. & n. s. & n. s. \\
\hline - Positive Orientierung (OP) & $0,09(0,29)$ & $0,17(0,42)$ & $0,14(0,39)$ & n. s. & n. s. & n. s. \\
\hline - Zielerreichung (ZE) & $0,48(0,51)$ & $0,17(0,42)$ & $0,20(0,44)$ & $\mathrm{T}$ & $*$ & n. s. \\
\hline - insgesamt & $3,35(1,23)$ & $2,74(1,34)$ & $3,08(1,23)$ & $\mathrm{T}$ & n. s. & n. s. \\
\hline
\end{tabular}

***/**/*/T $=1 \% 0 / 1 \% / 5 \% / 10 \%$-Niveau, n. s. = nicht signifikant, bei Produkte gefunden, Produkte gekauft $\chi^{2}$-Test nach Pearson, bei Alter Mann-Whitney-U-Test, sonst t-Test, 2seitig. sd in Klammern.

${ }^{1}$ Skala: von „1“ = sehr schlecht bis ,, $5 “=$ sehr gut.

${ }^{2}$ Skala: von „1“ - sehr unwahrscheinlich bis ,3“" - sehr wahrscheinlich 
Die Ergebnisse unserer Nachher-Befragung sind in Tabelle 5 und Tabelle 6 dargestellt. Bezogen auf drei Laufmuster, die aufgrund einer verdeckten Beobachtung ermittelt werden konnten, zeigen sich keine Altersunterschiede. In Bezug auf die Stimmung haben sich Stimmungsunterschiede und Stimmungsänderungen während des Kaufs nur im Vergleich von Cluster 2 und Cluster 3 ergeben. Unterschiede im kognitiven Geschehen zeigen sich im Vergleich dieser beiden Cluster sowie im Vergleich von Cluster 1 und Cluster 3, wenn auch nur bei wenigen Gedankenklassen. Schwach signifikante Gedankenunterschiede zeigen sich im Vergleich von Cluster 1 und Cluster 2. Und bezogen auf die Kauf-, Abteilungs- und Preisbeurteilung gab es ebenfalls nur einige wenige schwach signifikante Differenzen. Dasselbe gilt für die Absicht, die Abteilung vor Weihnachten noch einmal zu besuchen. Die Anzahl der Personen, die die gewünschten Produkte gefunden haben, fällt etwas niedriger aus als die Anzahl derer, die diese Produkte dann auch gekauft bzw. mitgenommen haben. Die Nennung von Gedanken hat nicht sehr viel Zeit in Anspruch genommen, denn pro Person wurden im Schnitt nur drei Gedanken benannt.

Bei der Beschreibung verschiedener Laufcluster anhand ausgewählter Gedankensequenzen in Tabelle 6 wurden solche Substrings ausgewählt, die relativ häufig festzustellen waren. Es handelt sich dabei um einfache Zweierketten. Es zeigt sich, dass sich in dieser Hinsicht die Laufmuster aber nur bezüglich der Abfolge „Intentionen/Ziele > Intentionen/Ziele“ (IZ > ON) sowie der Abfolge „Intentionen/Ziele > Orientierungsschwierigkeiten“ (IZ > IZ) (auf dem 5\%Niveau signifikant) unterscheiden. 
Tabelle 6: Beschreibung der Laufcluster anhand von Zweier-Sequenzen bei den Kognitionen

\begin{tabular}{|c|c|c|c|c|c|}
\hline & $\begin{array}{l}\text { Stichprobe } \\
\text { insgesamt } \\
\text { Häufigkeit; } \\
\text { Anteil }\end{array}$ & $\begin{array}{c}\text { Cluster } 1 \\
(\mathrm{n}=23) \\
\text { Häufigkeit; } \\
\text { Anteil }\end{array}$ & $\begin{array}{c}\text { Cluster } 2 \\
(\mathrm{n}=69) \\
\text { Häufigkeit; } \\
\text { Anteil }\end{array}$ & $\begin{array}{c}\text { Cluster } 3 \\
(\mathrm{n}=66) \\
\text { Häufigkeit; } \\
\text { Anteil }\end{array}$ & $\chi^{2}$-Tests \\
\hline$\overline{\mathrm{IZ} \rightarrow \mathrm{ON}}$ & $25 ; 23,4 \%$ & $3 ; 15 \%$ & $6 ; 17,6 \%$ & $16 ; 34,0 \%$ & $\chi^{2}(\mathrm{df}=2)=8,02 ; \mathrm{p}=0,018$ \\
\hline $\mathrm{IZ} \rightarrow \mathrm{IZ}$ & $21 ; 25,2 \%$ & $5 ; 25 \%$ & $4 ; 11,8 \%$ & $12 ; 25,5 \%$ & $\chi^{2}(\mathrm{df}=2)=7,40 ; \mathrm{p}=0,025$ \\
\hline $\mathrm{IZ} \rightarrow \mathrm{ZE}$ & $20 ; 18,7 \%$ & $4 ; 20 \%$ & $8 ; 23,5 \%$ & $8 ; 17,0 \%$ & $\chi^{2}(\mathrm{df}=2)=1,17 ; \mathrm{p}=0,558$ \\
\hline $\mathrm{IZ} \rightarrow \mathrm{AW}$ & $18 ; 16,8 \%$ & $5 ; 25 \%$ & $9 ; 26,5 \%$ & $4 ; 8,5 \%$ & $\chi^{2}(\mathrm{df}=2)=3,95 ; \mathrm{p}=0,139$ \\
\hline $\mathrm{IZ} \rightarrow \mathrm{AB}$ & $17 ; 15,9 \%$ & $3 ; 15 \%$ & $7 ; 20,6 \%$ & $7 ; 14,9 \%$ & $\chi^{2}(\mathrm{df}=2)=0,667 ; \mathrm{p}=0,716$ \\
\hline
\end{tabular}

IZ: Intentionen/Ziele; AW: Alternativen-Wahrnehmung; AB: Alternativenbewertung;

ON: Orientierungsschwierigkeiten; OP: positive Orientierung; ZE: Zielerreichung.

\section{Zusammenfassung und Ausblick}

Unser Vorschlag, die Verhaltensforschung am POS abzustufen und damit ein Methodenmix der besonderen Art zu praktizieren, geht von der Zielsetzung aus, möglichst viele Aspekte des POS-Verhaltens - das Laufverhalten, das Zuwendungs- und Kaufverhalten sowie kognitive Prozesse - mit möglichst hoher Aussagekraft und mit einem möglichst begrenztem Aufwand zu erfassen. Dabei fanden vor allem zwei Aspekte Beachtung: zum einen die Repräsentativität, d.h. die Selektivität und die Reaktivität, und zum anderen der Aufwand, der mit verschiedenen Erhebungen verbunden ist. Das abstufte Vorgehen geht deshalb mit einer abnehmenden Stichprobengröße einher. Dies entspricht letztlich auch dem Bemühen, die studienbedingte Beeinträchtigung von Ladenbesuchern so weit wie möglich zu begrenzen.

Die vorgeschlagene abgestufte Vorgehensweise in der Verhaltensforschung am POS empfiehlt sich nicht nur für die betriebliche und die kommerzielle Forschung, sondern auch für wissenschaftlich ambitionierte Forschungsarbeiten. In der konkreten Umsetzung eines abgestuften Vorgehens sind Varianten denkbar, die hier keineswegs umfassend dargestellt werden konnten. Welche Variante sich wann 
und wo anbietet, ergibt sich vor allem aus der Zielsetzung der Studie, der Ressourcenlage, der verfügbaren Technik (z.B. der verfügbaren Ortungssysteme), der verfügbaren Zeit und der Bereitschaft der Ladenbesucher, an einer Studie mitzuwirken. Das Fernziel wissenschaftlicher Studien könnte darin bestehen, eine Messtheorie zu entwickeln, die angibt, wann welches abgestufte Vorgehen mit welchem Aufwand welche Erkenntnisse zu Tage fördert. 

H

Die Erforschung des Mitarbeiterverhaltens am POS Ansatzpunkte und Methodik 


\section{Einleitung}

Bei aller Aufmerksamkeit, die dem Verhalten der Nachfrager bzw. Besucher eines Ladengeschäfts völlig zu Recht geschenkt wird, darf die Bedeutung des Mitarbeiterverhaltens für die Anbieterseite nicht unterschätzt werden. Das Verhalten der Mitarbeiter, sei es im Verkauf, sei es im Service, beeinflusst die Geschäftswahl bzw. die Geschäftstreue der Kunden und deren Verhalten im Geschäft, das Kaufverhalten eingeschlossen, und ist deshalb nicht nur für die Kunden von Bedeutung, sondern auch für den Erfolg des Geschäfts und der Hersteller der dort angebotenen Produkte.

Die Bedeutung der Mitarbeiter bzw. deren Einstellung und deren Verhalten den Kunden gegenüber konnte in den letzten Jahren immer öfter Gegenstand von Überlegungen und Abhandlungen in der Wissenschaft, aber auch in der Praxis werden. Doch was die Erfassung des Mitarbeiterverhaltens betrifft, dominiert nach wie vor eine recht einseitige Sicht - der Blick auf das beobachtbare Verhalten, das mit der sog. Testkaufmethode bzw. mit dem Einsatz von Testkäufern erfasst werden kann. Die Testkäufer werden oft, aber recht unzutreffend „mystery shopper“ oder „silent shopper“ genannt (Erstadt 1998, Wilson 1998, Calvert 2005), obwohl sie weder geheimnisvoll noch leise auftreten müssen. Wo die Forschung ansetzen kann, wenn es das offene, aber auch das verdeckte Mitarbeiterverhalten zu erfassen gilt, und wie dieser Einsatz zu beurteilen ist, soll im Folgenden näher betrachtet werden. Dabei ergibt sich die Gelegenheit, der Interaktion von Mitarbeitern und Kunden eine starke Aufmerksamkeit zu schenken und auch die Erfassung dieser Interaktion zu betrachten.

\section{Verhaltensebenen und Verhaltensbereiche}

Das Mitarbeiterverhalten (MV) kann als ein offenes, beobachtbares Verbalten interessieren, aber auch als inneres, nicht beobachtbares Verhalten. Bezüglich der Erfassungsmöglichkeiten kommen daher nicht nur die Beobachtungsmethoden in Frage, sondern auch alle anderen Methoden der Verhaltensforschung, das Erfragen 
von Gedanken und Emotionen inbegriffen. Da das Verhalten der Mitarbeiter ein dynamisches Geschehen darstellt, werden sich die folgenden Betrachtungen diesen Aspekt besonders berücksichtigen. Dies erklärt auch den Umstand, dass die Möglichkeiten der Einstellungs- und Zufriedenheitsmessung in diesem Beitrag weniger Beachtung finden.

Offene und verdeckte Verhaltenselemente auf der Seite der Mitarbeiter können recht unterschiedliche Sachverhalte umfassen. Dabei macht es einen Unterschied, welcher Verhaltensbereich angesprochen ist. Zu den wichtigsten Bereichen zählen erstens das Verhalten gegenüber den Kunden bzw. den Ladenbesuchern, die Mitarbeiter-Kunden-Interaktionen, zweitens das Verhalten gegenüber Vorgesetzten sowie anderen Mitarbeitern (Kollegen/innen) und drittens das Erledigen von Aufgaben, das keine sozialen Kontakte impliziert.

In Verkaufsgesprächen kommt dem Auftreten des Mitarbeiters, der Abfrage von Kundenwünschen, der Beratung in Bezug auf sinnvolle Ansprüche, den Informationen über Angebote und Konditionen, der Präsentation von Angeboten, dem Agieren im Verhandlungsprozess (Bargaining) und beim Abschließen sowie im Vorbereiten der Warenübergabe (Fulfillment) eine besondere Bedeutung zu. Und was das innere Verhalten angeht, können hier die Kundenwahrnehmung, das Einordnen des Kunden, das Zuschreiben bzw. die Attribution von Motiven und Verhaltensweisen sowie das Erleben von Erfolg oder Misserfolg und anderen Gefühlen wie Stress oder Anerkennung angeführt werden. Zu erwähnen ist auch das Dazulernen, sei es eine verbesserte Kundenkenntnis, sei es der Wissenszuwachs, der durch Hinweise des Kunden verursacht werden kann.

Bei Servicekontakten wie z.B. bei Reklamationen, Umtauschversuchen und der Bitte um einen Nachkaufservice ist daran zu denken, dass Mitarbeiter entgegenkommend bzw. helfend oder abweisend bzw. passiv reagieren können. Ggf. müssen sie mit der Aggression des Kunden, aber auch mit eigenen Gefühlen umgehen. Sie können aber auch wertvolle Hinweise erhalten und an den Verwendungserfahrungen der Kunden partizipieren. 
Ähnlich zeigt sich die Situation im Verhalten gegenüber Vorgesetzten und anderen Mitarbeitern. Zum offenen Verhalten zählt hier das bereitwillige Helfen und die Weitergabe von Wissen, das Verweigern von Hilfestellungen und der Teilung von Wissen, aber auch die Äußerung von Bewertungen jedweder Art. Bei erfahrenen Mitarbeitern kann die Aufgabe, den Nachwuchs auszubilden, hinzukommen. Als verdecktes Verhalten sind dementsprechend nicht nur die Arbeitsfreude, sondern auch der Arbeitsfrust zu nennen, auch der Einstieg in die innere Kündigung, womit der Verlust an Arbeitsfreude und der Verlust an Identifikation mit dem Unternehmen gemeint ist.

Als Aufgaben, die keine sozialen Kontakte implizieren, sind z.B. die Beantwortung von Anfragen per Post, per Fax oder per eMail, die Regalpflege, die Vorbereitung von Präsentationen, die Vorbereitung von Auslieferungen, die Bearbeitung von Reklamationen und das eigenständige Sichweiterbilden zu erwähnen. Dabei können dem Mitarbeiter ganz verschiedene Dinge durch den Kopf gehen, und auch die Emotionen, die dabei ausgelöst werden können, sind breit gefächert. Dieselbe Arbeit mag dem einen Mitarbeiter Freude bereiten, dem anderen dagegen eine große Last sein.

\section{Erfassungsmethoden und Bewertungskriterien im Überblick}

Das Verhalten der Mitarbeiter im Umgang mit Kunden, Vorgesetzten und Kollegen und das Verhalten bei der Vor- und Nachbereitung von Kundenkontakten lassen sich mit verschiedenen Methoden erfassen (vgl. van der Wiele et al 2005 S. 540). Beim offenen Verhalten kommen die Beobachtung, die Registrierung, die Videographie und die Befragung in Betracht, beim verdeckten Verhalten, z.B. bei den kognitiven Prozessen, die Befragung, die Denke-LautMethode (DLM) und die gestützte Rekonstruktion (GR). Bei vielen Sachverhalten ist die Kombination verschiedener Methoden sinnvoll, in bestimmten Fällen sogar unumgänglich.

Die Bewertung der Erfassungsmethoden sollte nicht nur auf deren grundsätzliche Aussage- bzw. Leistungskraft abstellen, sondern auch auf deren konkrete 
Anwendung in einer bestimmten Studie. Als klassische Standards kommen die Kriterien der internen und externen Validität in Frage. Bei der Beurteilung der internen Validität eines Messinstruments kennen wir zwei Kriterienklassen: zum einen die Reliabilitätskriterien als notwendige, aber nicht hinreichende Bedingungen, zum anderen die Validitätskriterien als notwendige und hinreichende Bedingungen. Werden erstere erfüllt, lohnt es sich, die letzteren in einem zweiten Schritt zu prüfen. Werden erstere nicht erfüllt, dann brauchen letztere nicht mehr geprüft werden. Dieses abgestufte Vorgehen ist deshalb ein ökonomisches Procedere, weil Validitätstests in aller Regel aufwendiger und schwieriger sind als die Reliabilitätstests.

$\mathrm{Zu}$ den klassischen Reliabilitätskriterien zählt die Objektivität, die Übereinstimmung von Test- und Nachtestergebnissen (Retestreliabilität) und die Korrelation von Messergebnissen an solchen Messpunkten, die alle dasselbe abzubilden vorgeben (Indikatorenkorrelation). Zu den klassischen V aliditätskriterien zählen die Augenscheinvalidität, die am Urteil eines Experten festmacht, die Kriteriumsvalidität, die am Messergebnis eines geeigneten Prüfkriteriums festmacht, die Konvergenzvalidität, die sich aus dem Vergleich mit einem anderen, bewährten Messinstrument ergibt, und die nomologische Validität, die sich an den Aussagen einer guten Messtheorie orientiert. Kann die interne Validität und damit die Aussagekraft der Befunde vorausgesetzt werden, so ist noch zu klären, ob die Befunde auf größere Populationen und andere, vergleichbare, nicht untersuchte Einkaufsaktivitäten schließen lassen. Ist dies der Fall, so kann von einer Generalisierbarkeit bzw. von einer externen Validität der Befunde gesprochen werden.

\section{Einzelne Erfassungsmethoden und deren Bewertung}

Die Darstellung einzelner Erfassungsmethoden und deren Bewertung sollen sich im Folgenden vor allem auf die Erforschung des Mitarbeiterverhaltens im Umgang mit dem Kunden - sei es im Verkauf, sei es im Service - konzentrieren. Ein 
Großteil der dabei getroffenen Aussagen lässt sich jedoch auch auf die Erfassung des Mitarbeiterverhaltens in anderen Verhaltensbereichen übertragen.

\subsection{Beobachtungsmethoden und deren Eignung}

Das offene Verhalten der Mitarbeiter im Umgang mit dem Kunden ist einer verdeckten und einer offenen Beobachtung zugänglich. Die Testkauf- bzw. Testkäufermethode ist eine häufig praktizierte Variante der verdeckten Beobachtung. In solchen Fällen versuchen ausgewählte und geschulte Personen als „normale Käufer" aufzutreten und das Verhalten des kontaktierten Mitarbeiters oder mehrerer Mitarbeiter zu registrieren, ggf. auch zu bewerten, und dabei als Tester bzw. Forscher nicht erkannt zu werden.

Ein Beobachter könnte sich jedoch auch als solcher offen zu erkennen geben und das Verhalten der Mitarbeiter im alltäglichen Umgang mit „echten“ Kunden festzuhalten und ggf. auch zu bewerten. In diesem Falle wüsste der angesprochene Mitarbeiter um die geplante Beobachtung und könnte diese u.U. sogar ablehnen. Im Falle einer Zustimmung wüsste er um die Beobachtung und könnte sein Verhalten den vorgegebenen oder vermuteten Normen entsprechend ausrichten.

Hat ein Testkäufer nicht nur die Aufgabe, den von ihm angesprochenen Verkäufer und dessen Verhalten zu analysieren, sondern auch andere Mitarbeiter in ihrem Umgang mit anderen Kunden zu beobachten (z.B. in der Studie von Finn \& Kayandé 1999 S.209), findet insofern eine Methodenkombination statt, als es sich in Bezug auf den angesprochenen Mitarbeiter um eine teilnehmende Beobachtung handelt und in Bezug auf die anderen Mitarbeiter um eine nicht-teilnehmende Beobachtung.

Ein Beobachter könnte auch die Käuferrolle übernehmen und den darüber informierten Mitarbeiter in seinem Verhalten studieren. Dabei handelte es sich im Grunde um die Simulation eines Verkaufsgesprächs oder eines Servicekontakts. Solche Fälle sind vor allem als Mitarbeiter- bzw. Verkäufertrainings denkbar (vgl. Bromage 2000). 
Das Besondere an einer offenen Beobachtung von Mitarbeitern liegt darin, dass der Inhaber des Ladengeschäfts seine Mitarbeiter als Arbeitgeber auffordern kann, an einer solchen Studie teilzunehmen. Der Inhaber oder der Vorgesetzte kann seine Mitarbeiter aber auch davon unterrichten, dass eine verdeckte Beobachtung stattfinden wird. Eine nach Ort und Zeit konkretisierte Ankündigung empfiehlt sich angesichts der Gefahr, dass die Mitarbeiter ihr Verhalten gezielt und zeitlich begrenzt ändern, jedenfalls nicht. Sind Testkaufstudien oder vergleichbare Erhebungen vorgesehen, ist es in aller Regel sinnvoll, wenn nicht gar geboten, die Mitarbeiter vorher zu unterrichten und dabei auch zu sagen, zu welchen Zwecken eine solche Erhebung stattfinden soll (vgl. Punch 1994, Wilson 2001 S.729).

Wilson (2001 S.731) verweist noch auf eine Möglichkeit des Testkäufereinsatzes, bei der nicht einmal der Inhaber bzw. das Management des untersuchten Ladengeschäftes oder der untersuchten Ladenkette informiert werden müssen: die Einrichtung eines Verbraucherpanels, bei dem die Panelmitglieder über einen längeren Zeitraum hinweg über das Verhalten des Verkaufspersonals in jenen Geschäften berichten, die sie bei ihren alltäglichen Einkäufen aufsuchen.

Soweit sich die Forschung zur Eignung der Beobachtung von Mitarbeitern im Umgang mit Kunden befasst hat, betrifft dies allein die Testkaufmethode. Und selbst bei dieser Methode sind nur selten Hinweise zu ihrer Aussagekraft zu finden, obwohl sie in der Praxis recht häufig und immer häufiger eingesetzt wird (Finn \& Kayandé 1999 S.196). Einigkeit besteht darüber, dass die interne Validität der Feststellungen eingesetzter Testkäufer entscheidend von deren Eignung, deren Ausbildung und ihrer Einweisung in die jeweilige Aufgabe abhängt. Die Testkäufer müssen nicht nur gezielt agieren, gezielt beobachten, das Beobachtete sich merken und später, nach dem Geschäftsbesuch, richtig festhalten bzw. berichten können (Hesselink \& van der Wiele 2003). Diese schwierige Aufgabe hat bei einer Testkaufstudie in Reisebüros dazu geführt, dass zwei Beobachter als Team eingesetzt wurden (Lingenfelder et al. 2003). Eine solche Praxis eröffnet im Übrigen die Möglichkeit, die Konsistenz der Berichte beider Testkäufer als 
Reliabilitätskriterium heranzuziehen (s. dazu die Inter-Rater-Reliabilität bei Lingenfelder et al. 2003 S.297). Zur internen Validität der Testkaufmethode vertreten mehrere Autoren zudem die Auffassung, dass die über geeignete Testkäufer gewonnenen Beobachtungsergebnisse valider sind als die Ergebnisse von Befragungen, in denen Verkäufer über ihren Umgang mit Kunden Auskunft geben sollten.

Testkaufstudien sind vor allem in ihrer gängigen Auslegung bzw. in ihrer externen Validität gefährdet. Damit ist die Tendenz angesprochen, von den Ergebnissen auf den alltäglichen Umgang der Mitarbeiter mit ihren Kunden zu schließen. Die Generalisierbarkeit kann zum einen unter der Selektivität des Testkäufereinsatzes, zum anderen aus der Reaktivität der ,getesteten Mitarbeiter“ leiden.

Zur Selektivität einer Testkäuferstudie tragen vor allem folgende Faktoren bei: der ausgewählte Zeitpunkt, die ausgewählten Geschäfte oder Abteilungen, die ausgewählten Mitarbeiter, das Verhalten, das der Testkäufer zeigen soll, und die Aspekte des Mitabeiterverhaltens, die der Testkäufer registrieren soll (vgl. Finn \& Kayandé 1999 S.198, Finn 2001 S.313). Finn \& Kayandé (1999 S.196) betonen, dass die Testkäufer für die Kundschaft eines Geschäfts - also auch für deren Verhalten - repräsentativ sein sollten. Entsprechende Studien zeigen, dass bei Testkaufstudien weit mehr Testkäufer als gemeinhin üblich (etwa drei pro Geschäft) eingesetzt werden müssen, wenn eine Generalisierbarkeit der Befunde auf der Ebene eines Geschäfts oder der Ebene einer Ladenkette erreicht werden soll (Finn \& Kayandé 1999 S.199, Finn 2001 S.316).

Die Reaktivität der Testkaufmethode hat bislang ebenfalls wenig Beachtung finden können. Eine mögliche Erklärung dafür liegt in der impliziten Annahme, dass eine verdeckte Beobachtung definitionsgemäß nicht bemerkt wird. Eine solche Annahme verkennt jedoch die Tatsache, dass eine verdeckte Beobachtung unbemerkt bleiben soll, aber keineswegs unbemerkt bleiben muss. Außerdem wird dabei verkannt, dass die Reaktivität eines Verkäufers auch dann entstehen kann, 
wenn er eine Testkauf-Situation bzw. eine entsprechende verdeckte Beobachtung als solche lediglich vermutet. Und schließlich kann Reaktivität auch dann entstehen, wenn das Beobachtetwerden im Rahmen einer Testkaufstudie lediglich als seltenes, aber alltägliches Verhalten eines Kunden wahrgenommen wird.

Weiß ein Mitarbeiter, dass er zu Kontrollzwecken bzw. im Rahmen einer Testkaufstudie beobachtet wird, wird er versuchen, sein Verhalten den Vorgaben oder den vermuteten Normen entsprechend besonders gut auszurichten - einen besonders guten Mitarbeiter darzustellen (vgl. Finn \& Kayandé 1999). Dasselbe gilt dann, wenn er lediglich vermutet, im Rahmen einer Erhebung oder eines Tests beobachtet zu werden. Jedenfalls kann die Reaktion auf die Ankündigung eines Testkäuferprogramms und auf deren auf Dauer angelegte Realisierung nicht nur vorteilhaft, sondern auch bezweckt sein, wenn es um einen verbesserten Umgang mit den Kunden und um zielführende Verkaufsgespräche geht (zum motivationalen Aspekt der Testkaufmethode s. Erstadt 1998, Zeldis 1998).

Erste Befunde zur Wahrnehmung eines Testkäufers in seiner Rolle als Testkäufer liefert die Befragungsstudie in GB von Douglas et al. (2007). Befragt wurden 75 Mitarbeiter einer Handelskette, die sich für ein Testkäuferprogamm entschieden hatte. $84 \%$ der Befragten wussten um dieses Programm und $64 \%$ wussten auch um die Beurteilungskriterien, die den Testkäufern vorgegeben waren. $4 \%$ gaben an, die Testkäufer als solche schon dann zu erkennen, wenn sie das Geschäft betreten. Die Beurteilung fiel zwiespältig aus: $20 \%$ sagten, dass das Programm das Verhalten bzw. die Leistung der Mitarbeiter stark verbessere, $77 \%$ sprachen von einer kleinen Verbesserung. Aber $84 \%$ gaben auch an, dass das Programm eine Quelle für Stress und Angst darstelle (vgl. dazu auch Erstadt 1998).

Ein Jahr später führten Douglas et al. (2008) eine vergleichbare Studie bei derselben Handelskette in GB durch. Insgesamt konnten 98 Fragebogen ausgewertet werden. Vermutlich waren es nur teilweise dieselben Leute, weil vor allem bei den Teilzeitkräften eine hohe Fluktuation vorherrschte. Die Ergebnisse dieser zweiten Umfragestudie reflektieren jedenfalls den erweiterten 
Erfahrungshorizont der Befragten. $95 \%$ der Befragten konnten von einer Teilnahme an einem Trainingsprogramm berichten. $70 \%$ hatten vorgegebene (Service-)Standards zu beachten. $68 \%$ wussten um die Kriterien, die bei ihrer Bewertung durch die Testkäufer zur Anwendung kommen. Jetzt gaben immerhin $34 \%$ - also ein Drittel der Befragten - an, also weitaus mehr als ein Jahr zuvor, einen Testkäufer als solchen erkennen zu können. Dies ließe sich an bestimmten Sachverhalten ablesen, nämlich an den gestellten Fragen (von $45 \%$ genannt), an der Anwesenheit einer Person, die die Testkäufer beobachtet (dem „over observant $^{*}$ ) (von $24 \%$ genannt), am wiederholten Besuch der betreffenden Person (von $12 \%$ genannt) und am letztendlichen Ausbleiben eines Kaufes (von $9 \%$ genannt). Die Beurteilung der Testkaufmethode fiel wiederum zwiespältig aus: 47 $\%$ sagten, dass das Programm eine Motivationssteigerung bewirke, $22 \%$ stellten einen negativen Effekt und 31 \% gar keinen Effekt fest. Jetzt waren es immerhin $60 \%$, die sich dann gestresst oder beängstigt fühlen, wenn ein Testkäuferbesuch ansteht.

Wenn wir nun die skeptische und kritische Einstellung vieler Mitarbeiter gegenüber Testkaufstudien als gegeben annehmen, stellt sich die Frage, ob die negativen Angaben in einer Umfragestudie nicht auch aus der Absicht resultieren, mit besonders negativen Angaben wie z.B. der geringen Wirksamkeit und der Erkennbarkeit von Testkäuferbesuchen zur Aufgabe entsprechender Programme beizutragen. Träfe dies zumindest bei einem Teil der Befragten zu, wäre davon auszugehen, dass Umfragebefunde in diesem Kontext und damit auch die Ergebnisse bei Douglas et al. (2007, 2008) die kritischen Aspekte der Testkaufmethode mehr oder weniger stark überzeichnen.

\subsection{Registrierungsmethoden und deren Eignung}

Der Umgang von Mitarbeitern mit den Kunden kann sich in Spuren oder Dokumenten niederschlagen und so einer Registrierung zugänglich sein. Dabei ist vor allem an Reaktionen auf Anfragen oder Forderungen oder Kritik zu denken, die über Telekommunikationssysteme (Telefon, Fax, SMS), über die klassische Post 
(Briefe, Karten) oder über das Internet (eMails) beantwortet werden. Dabei macht es einen Unterschied, ob die Dokumente und Spuren dem Kunden, einem Testkäufer bzw. Testanfrager bzw. Testkritiker und/oder internen Stellen zugänglich sind. Die Testkäufermethode lässt sich hier ebenso anwenden wie die Bitte an „echte“ Kunden, über die Reaktionen der Mitarbeiter zu berichten. Dass dabei das Kommunikationsverhalten und ggf. auch die vom Mitarbeiter erbrachte oder auch nur initiierte Leistung in Erfahrung gebracht werden können, nicht jedoch deren verdecktes Verhalten, versteht sich von selbst.

Intern lässt sich z.B. die Nutzung von Informationssystemen durch die Mitarbeiter registrieren. Dies könnte gezielt für solche Nutzungsaktivitäten erfolgen, die den Kontakt mit Kunden erleichtern oder verbessern helfen. Zu denken wäre hier zum einen an das Registrieren des Abfrageverhaltens in einem Kundeninformationssystem, das z.B. per Intranet online zugänglich ist, zum anderen die Auswertung jener Logfiles, die anfallen, wenn ein Laptop als Unterstützung von Verkaufs- oder Servicegesprächen, auch als Konfigurationssysteme, offline zum Einsatz kommen (zum Laptopeinsatz im Verkaufsgespräch s. Silberer \& Kretschmar 1999, insbes. S.23, 200). Sind die dabei anfallenden Aufzeichnungen, z.B. die serverseitigen Logfiles, bei Online-Abfragen wegen der Proxy- und Cache-Problematik nicht vollständig, müsste ggf. auf die „clientseitige“ Logfile-Aufzeichnung und -Auswertung gesetzt werden. Jedenfalls wäre hier eine Zustimmung der betroffenen Mitarbeiter erforderlich, zumindest hilfreich.

\subsection{Videographische Methoden und deren Eignung}

Videoaufnahmen, die ein bestimmtes Verhalten festhalten und zeigen, haben nicht nur den Vorteil der Anschaulichkeit, vor allem der Berücksichtigung verbaler und nonverbaler Inhalte, sondern auch den Vorteil, dass sie einer sorgfältigen Auswertung zur Verfügung stehen. Sie stehen auch wiederholten Auswertungen zur Verfügung, die ggf. mit veränderten Fragestellungen an das Material 
herangehen. Sie können auch eine gute Grundlage abgeben, wenn die Aufzeichnung der handelnden Personen besprochen werden soll.

Soll das Verhalten von Mitarbeitern im Umgang mit Kunden aufgezeichnet werden, so muss bei beiden Seiten oder zumindest beim Kunden die Zustimmung erbeten und erhalten werden. Übernimmt der Forscher oder ein anderer die Kundenrolle, ist dieses mögliche Hindernis aus dem Weg geräumt. Finden z.B. im alltäglichen Geschehen verdeckte Aufnahmen von Kundenkontakten statt, sind beide Seiten im Nachhinein aufzuklären bzw. zu informieren. Die beteiligten Kunden müssen außerdem einer weiteren Verwendung bzw. Auswertung der Aufnahmen zustimmen.

So aufschlussreich die Resultate einer Videographie und so gut die Möglichkeiten einer Auswertung von Videos auch sein mögen, so ist doch zu bedenken, dass die Teilnehmer bei den entsprechenden Studien immer dann eine Selektion darstellen, wenn deren Zustimmung erforderlich ist. Außerdem können die Teilnehmer zu Reaktivität neigen, d.h. ein Verhalten zeigen, das dem Verhalten, bei dem keine Filmaufnahmen gemacht werden, nicht ganz oder überhaupt nicht entspricht. Vermutlich ist bei Mitarbeitern die Gefahr, sich in einem guten Licht zeigen zu wollen, viel stärker und außerdem anders ausgeprägt als bei den Kunden.

\subsection{Befragungsmethoden und deren Eignung}

Sind Beobachtungen aus zeitlichen, räumlichen oder rechtlichen Gründen nicht möglich oder von Seiten der Beteiligten nicht erwünscht, ist deren Befragung zu erwägen. Zu erfragen wäre das Verhalten der Mitarbeiter im Umgang mit den Kunden. Was die kognitiven Prozesse bei den Mitarbeitern angeht, ist die Befragung der Mitarbeiter ohnehin die Methode der Wahl, die klassische Alternative.

Da es um das Verhalten der Mitarbeiter im Umgang mit Kunden geht, könnten grundsätzlich beide Seiten befragt werden - die Mitarbeiter zu ihrem Verhalten und die Kunden zum Verhalten der Mitarbeiter. Dies eröffnet die Möglichkeit, Aussagen beider Seiten zu vergleichen und diskrepanten Aussagen gezielt 
nachzugehen. Allerdings impliziert eine solche Spiegelbild-Befragung, dass beide Seiten, also auch die Kunden, zu Aussagen bereit sind.

Bezogen auf einzelne Kundenkontakte ist zu überlegen, ob der Mitarbeiter und/oder der Kunde sofort nach dem Kontakt befragt werden sollen. Dies hätte den Vorteil, dass sich die Vergessensprozesse in Grenzen halten. Der Mitarbeiter könnte berichten, was er gerade getan hat und was ihm dabei durch den Kopf gegangen ist. Auch die erlebten Gefühle kann er beschreiben. Und der Kunde kann berichten, welches Verhalten er beim Mitarbeiter wahrgenommen hat. Kann eine Befragung erst viel später stattfinden, sei es beim Mitarbeiter, sei es beim Kunden, ist zu erwarten, dass nur bestimmte Verhaltensweisen, Gedanken und Gefühle erinnert werden - zum einen die ohnehin üblichen, zum anderen die ganz außergewöhnlichen.

Ähnliches gilt, wenn ein Mitarbeiter über mehrere Kundenkontakte hinweg berichten soll, seien es viele Kontakte mit mehrere Kunden, seien es mehrere Kontakte mit ganz bestimmten Kunden. Hier sollten keine umfassenden und detaillierten Berichte erwartet werden - eher nur Hinweise auf das Übliche, vielen Kontakten Gemeinsame, und auf das Außergewöhnliche, so z.B. seltene Wahrnehmungen und besonders starke Emotionen. So könnte ein Mitarbeiter z.B. nach besonders schwierigen oder besonders erfreulichen, nach besonders erfolgreichen und weniger erfolgreichen Gesprächen gefragt werden. Soll dagegen ein Kunde über mehrere Kontakte mit bestimmten Mitarbeitern berichten, kann vielleicht eine bessere Erinnerungsleistung erwartet werden. Dies setzt allerdings voraus, dass solche Kontakte stattgefunden haben und dass die überdurchschnittlich häufigen Kontakte beim Kunden eine gewisse Aufmerksamkeit erzielen konnten. In diesem Zusammenhang ließe sich ein Kunde z.B. fragen, wie die Gespräche mit einem häufig kontaktierten Mitarbeiter verlaufen sind. Es könnte auch interessieren, wann welche Mitarbeiter besonders freundlich waren oder sich besonders viel Mühe gegeben haben. 
Die Denke-Laut-Methode als Möglichkeit, Gedanken ohne Vergessenslücken zu erfassen, kommt bei Kundenkontakten i.d.R. nicht in Frage, auch nicht bei Telefongesprächen (zum Einsatz dieser Methode in der Käuferforschung siehe Payne \& Ragsdale 1978, Titus \& Everett 1996 sowie Reiks et al. 2003). Vergessenslücken können aber dadurch reduziert werden, dass eine gestützte Gedankenabfrage stattfindet. Dazu mehr im nächsten Abschnitt.

\subsection{Die gestützte Erinnerung des Verhaltens}

Lassen sich das offene Verhalten sowie die Gedanken und Gefühle der Mitarbeiter im Umgang mit dem Kunden nicht unverzüglich erfragen, muss es darum gehen, bei einer späteren Befragung Erinnerungshilfen einzusetzen. Die Frage nach möglichen Erinnerungsstützen bei einer späteren Abfrage von Verhaltensweisen, Gedanken und Gefühlen lässt sich für Gespräche, Telefonate und Mailkontakte unterschiedlich beantworten.

Was einem Mitarbeiter beim Gespräch mit einem Kunden durch den Kopf ging und was er dabei empfunden hat, lässt sich ggf. durch Gesprächprotokolle, durch Notizen eines Beobachters oder durch Tonband- oder Videoaufzeichnungen gestützt abfragen und somit rekonstruieren (zur videogestützten Gedankenrekonstruktion in der Käuferforschung s. Silberer 2005, 2008, Silberer \& Büttner 2008, Büttner 2008). Bei einem Verkaufsgespräch könnte ggf. allein schon ein Foto vom Gesprächspartner genügen, um beim betroffenen Mitarbeiter bestimmte Erinnerungen wachzurufen. Bandaufzeichnungen oder die vom Kunden erinnerten Inhalte bieten sich bei Telefongesprächen an, soweit die Betroffenen einer Aufzeichnung zustimmen konnten und der Kunde entsprechende Angaben machen konnte. Was einem Mitarbeiter beim Verfassen eines Briefes oder einer Mail durch den Kopf ging, lässt sich besser erinnern, wenn die Kopie des Schreibens, ggf. auch die Kopie des Antwortschreibens, vorgelegt werden kann. Und was einem Mitarbeiter beim PC-gestütžten Verkaufsgespräch, sei es offline, sei es online, durch den Kopf ging, lässt sich ggf. über die Rekonstruktion der Antwort- bzw. 
Seitenabfolge bzw. der entsprechenden Logfiles besser rekonstruieren als ohne derartige Erinnerungshilfen.

\section{Zum simultanen und konsekutiven Methoden-Mix}

Der kombinierte Einsatz unterschiedlicher Methoden zu ein und demselben Zeitpunkt bzw. in Rahmen eines bestimmten Untersuchungsvorhabens sei hier als „simultanes Methoden-Mix“ bezeichnet. Den zeitlich versetzten Einsatz verschiedener Methoden bezeichnen wir dagegen als ein „konsekutives Methoden-Mix“.

Zum simultanen Methoden-Mix zählen folgenden Fälle:

(1) Ein Beobachter verfolgt ein Verkaufsgespräch und befragt anschließend beide Seiten zu ihrem Verhalten sowie zu den begleitenden Gedanken und Empfindungen.

(2) Ein Beobachter zeichnet ein Verkaufsgespräch per Video auf und nutzt das Video anschließend als Erinnerungsstütze bei der Rekonstruktion begleitender Gedanken.

(3) Ein Beobachter befragt einen Mitarbeiter vor einem Verkaufsgespräch in Bezug auf die Vorbereitung des anstehenden Gesprächs und beobachtet ihn dann während des Gesprächs, um nach dem Gespräch die kritischen Punkte mit dem betreffenden Mitarbeiter zu besprechen.

Auch für den Bereich des konsekutiven Methoden-Mix sollen hier einige Bespiele genügen:

(1) Eine Mitarbeiterbefragung interessiert sich für die Erfahrungen der Mitarbeiter mit verschiedenen Kunden, die über einen längeren Zeitraum gemacht werden konnten. Danach findet eine Beobachtung ausgewählter Kundengespräche statt, z.B. jener Gespräche, bei denen sich die Kunden in der Vergangenheit als besonders schwierig erwiesen hatten.

(2) Eine Kundenbefragung interessiert sich für die Erfahrungen, die die Befragten mit bestimmten Mitarbeitern über einen längeren Zeitraum gemacht haben. 
Danach findet eine Beobachtung ausgewählter Kundengespräche statt, z.B. jener Gespräche, bei denen sich die Mitarbeiter aus Kundensicht als wenig hilfsbereit erwiesen hatten.

(3) Ein Beobachter verfolgt die Verkaufsgespräche ausgewählter Mitarbeiter mit den verschiedensten Kunden über einen längeren Zeitraum hinweg und befragt danach die beobachteten Mitarbeiter zu allen untersuchten Kundengesprächen.

Anhand derartiger und anderer Methodenkombinationen kann versucht werden, die Vorteile einzelner Methoden in einer Art Arbeitsteilung oder Synergiestrategie zu nutzen. Daneben könnte aber auch versucht werden, das Leistungspotenzial einzelner Methoden zu vergleichen und diese einer Art Vergleichstest zu unterwerfen.

\section{Zusammenfassung und Ausblick}

Ein Überblick über die verschiedensten Ansatzpunkte und Methoden, die bei einer Analyse des Mitarbeiterverhaltens in Ladengeschäften in Betracht kommen, macht deutlich, dass der viel besprochene Testkäufer- oder Mystery Shopper-Einsatz nur eine von vielen relevanten Methoden darstellt und auch nur einen Teil des relevanten Verhaltens, das offene, erfassen kann. Bei der Auswahl einzelner Methoden und ihrer Kombination ist nicht nur darauf zu achten, was jedes einzelne Instrument auf Grund seiner internen Validität zu Tage fördern kann, sondern auf die möglichen Selektivitäts- und Reaktivitätseffekte. Dasselbe gilt für den rechtlichen und ethischen Rahmen und für die zu erwartende Akzeptanz seitens der betroffenen Personen bzw. Personenkreise. Kommt es künftig zu einer sorgfältigen, breit angelegten Analyse des Mitarbeiterverhaltens, dann steigt auch die Chance, dass die Formulierung einer guten Theorie der Mitarbeiter-KundenInteraktion gelingt und somit eine wertvolle Grundlage für Entscheidungen im Anbieterbereich, z.B. in der Auswahl, Ausbildung und Führung von Mitarbeitern, liefert. 


\section{Literatur}

Bromage, N. (2000).

Mystery Shopping - It's Research, But Not as We Know It, in: Management Accounting, Vol. 78 (2000), No 4, p. 30

Büttner, O. (2008).

Die Untersuchung einkaufsbegleitender kognitiver Prozesse am Point of Sale Eine Arbeit zur Qualität von Datenerhebungsmethoden der Konsumentenforschung, Dissertationsschrift, Universität Göttingen, Wirtschaftswissenschaftliche Fakultät

Calvert, P. (2005).

It's a Mystery: Mystery Shopping in New Zealand's Public Libraries, in: Library Review, Vol. 54 (2005), No 1, pp. 24-35

Douglas, A., Douglas, J. \& Davies, J. (2007).

The Impact of Mystery Customers on Employees, in: Our Dreams of Excellence, $10^{\text {th }}$ Conference on Quality Management and Organizational Development, Helsingborg, Sweden, 18-20 June, 2007, Linköping Electronic Conference Proceedings, Linköping University Electronic Press, Article No 55

Douglas, A., Douglas, J. \& Davies, J. (2008).

The Effect of Performance Measurement on People, in: Proceedings of the $13^{\text {th }}$ International Conference on Quality and Productivity Research (ICQRP), Oulu, Finland 2008, pp. 167-178

Erstadt, M. (1998).

Mystery Shopping Programmes and Human Resource Management, in: International Journal of Contemporary Hospitality Management, Vol. 10 (1998), No 1, pp. 34-38

Finn, A. (2001).

Mystery Shopper Benchmarking of Durable-Goods Chains and Stores, in: Journal of Service Research, Vol. 3 (2001), No 4, pp. 310-320

Finn, A. \& Kayandé, U. (1999).

Unmasking a Phantom: A Psychometric Assessment of Mystery Shopping, in: Journal of Retailing, Vol. 75 (1999), No 2, pp. 195-217

Hesselink, M. \& van der Wiele, T. (2003).

Mystery Shopping: In-depth Measurement of Customer Satisfaction, in: ERIMReport Series Research in Management, ERS-2003-20-ORG, March 2003 
Lingenfelder, M., Wieseke, J. \& Schmidt, K. (2003).

Dienstleistungsqualität von Reisebüro-Unternehmen. Modellierung und Messung mittels Mystery Shopping - Am Fallbeispiel einer im Franchisingsystem organisierten Reisebürokette, in: Tourismus Journal, 7. Jg. (2003), Heft 3, S. 283306

Payne, J.W. \& Ragsdale, E. K. E. (1978).

Verbal protocols and direct observation of supermarket shopping behaviour: Some findings and a discussion of methods, in: H. K. Hunt (Ed.). Advances in Consumer Research, Chicago: Association for Consumer Research, Vol. 5, pp. 571-577

Punch, M. (1994).

Politics and Ethics in Qualitative Research, in: N. K. Denzin \& Y. S. Lincoln (Eds). Handbook of Qualitative Research, London: Sage, pp. 83-97

Reicks, M., Smith, C., Henry, H., Renier, K., Atwell, J. \& Thomas, R. (2003).

Use of the Think Aloud Method to Examine Fruit and Vegetable Purchasing Behaviours among Low-Income African American Women, in: Journal of Nutrition Education and Behaviour, Vol. 35 (May 2003), No 3, pp. 154-163

Silberer, G. (2005).

Die videogestützte Rekonstruktion kognitiver Prozesse beim Ladenbesuch, in: Marketing. ZFP, Vol. 27 (2005), Heft 4, S. 263-271

Silberer, G. (2008).

Behavior at the POS - Classical and Newer Methods of Recording it, in: T. Lowry (Ed.), Brick \& Mortar Shopping in the 21st Century, Mahwah, NJ: Lawrence Erlbaum, pp. 257-280

Silberer, G. \& Büttner O. (2008).

Marktforschung am Point of Sale, in: A. Herrmann, C. Homburg \& M. Klarmann (Hg.). Handbuch Marktforschung, 3. Aufl., Wiesbaden: Gabler, S. 1097-1123

Silberer, G. \& Kretschmar, C. (1999).

Multimedia im Verkaufsgespräch. Mit zehn Fallbeispielen für den erfolgreichen Einsatz, Gabler: Wiesbaden

Titus, Ph. A. \& Everett, P. B. (1996).

Consumer Wayfinding Tests, Strategies and Errors: An Exlaboratory Field Study, in: Psychology \& Marketing, Vol. 13 (1966), No 3, pp. 265-290

Van der Wiele, T., Hesselink, M. \& van Iwaarden, J. (2005).

Mystery Shopping: A Tool to Develop Insight into Customer Service Provision, in: Total Quality Management, Vol. 16 (2005), No 4, pp. 529-541 
Wilson, A. M. (1998).

The Role of Mystery Shopping in the Measurement of Service Performance, in: Managing Service Quality, Vol. 8 (1998), No 6, pp. 414-420

Wilson, A. M. (2001).

Mystery Shopping: Using Deception to Measure Service Performance, in: Psychology \& Marketing, Vol. 18 (2001), No 7, pp. 721-734

Zeldis, N. (1988).

The Phantom Shoppers Strike Again and Again, in: Management Review, June 1988, pp. 10-11 
/iele Kaufentscheidungen fallen erst am Point of Sale (POS), zumindest werden viele Absichten dort erst konkretisiert. Dies allein begründet die herausragende Bedeutung der Verhaltensforschung am POS. Bei dieser Forschung bedarf es des Einsatzes geeigneter Forschungsmethoden und der Beachtung ihrer Leistungsfähigkeit. Aus diesem Grunde befasst sich die hier vorgelegte Publikation mit den klassischen, aber auch mit neueren Forschungsmethoden, vor allem mit der Erfassung des Lauf-, Zuwendungs- und Kaufverhaltens und mit der Erfassung kognitiver Prozesse beim Besuch eines stationären Geschäfts. Besondere Beachtung erfahren Validitätsaspekte sowie Selektivitäts- und Reaktivitätseffekte, die über die Aussagekraft der Befunde entscheiden. Um aufzuzeigen, wie mit begrenztem Aufwand möglichst viel in Erfahrung gebracht werden kann, wird eine abgestufte Verhaltensforschung am POS skizziert. Abschließend wird aufgezeigt, wie auch das Verhalten der Mitarbeiter am POS untersucht werden kann. 